\title{
The Recognition Theorem for Graded Lie Algebras in Prime Characteristic
}

\author{
Georgia Benkart* \\ Thomas Gregory \\ Alexander Premet
}

\author{
Author address: \\ Department of Mathematics, University of Wisconsin-Madison, \\ MADison, Wisconsin 53706 USA \\ E-mail address: benkart@math.wisc.edu \\ Department of Mathematics, The Ohio State University at \\ Mansfield, Mansfield, OHIO 44906 USA \\ E-mail address: tgregory@math.ohio-state.edu \\ School of Mathematics, The University of Manchester, \\ MANCHESTER M13 9PL, UK \\ E-mail address: sashap@maths.man.ac.uk
}





\section{Contents}

Introduction vii

Chapter 1. Graded Lie Algebras 1

1.1. Introduction 1

1.2. The Weisfeiler radical 2

1.3. The minimal ideal $\mathfrak{J} \quad 4$

1.4. The graded algebras $\mathcal{B}\left(\boldsymbol{V}_{-\boldsymbol{t}}\right)$ and $\mathcal{B}\left(\boldsymbol{V}_{\boldsymbol{t}}\right) \quad 5$

1.5. The local subalgebra $\quad 8$

1.6. General properties of graded Lie algebras 9

1.7. Restricted Lie algebras $\quad 15$

1.8. The main theorem on restrictedness (Theorem 1.63) 17

1.9. Remarks on restrictedness 17

1.10. The action of $\mathfrak{g}_{\mathbf{0}}$ on $\mathfrak{g}_{-\boldsymbol{j}} \quad 18$

1.11. The depth-one case of Theorem $1.63 \quad 20$

1.12. Proof of Theorem 1.63 in the depth-one case 21

1.13. Quotients of $\mathfrak{g}_{0} \quad 22$

1.14. The proof of Theorem 1.63 when $\mathbf{2} \leq \boldsymbol{q} \leq \boldsymbol{r} \quad 24$

1.15. The proof of Theorem 1.63 when $\boldsymbol{q}>\boldsymbol{r}$

1.16. General setup $\quad 25$

1.17. The assumption $\left[\left[\mathfrak{g}_{-1}, \mathfrak{g}_{1}\right], \mathfrak{g}_{1}\right] \neq 0$ in Theorem 1.63

Chapter 2. Simple Lie Algebras and Algebraic Groups 33

2.1. Introduction 33

2.2. General information about the classical Lie algebras 33

2.3. Representations of algebraic groups 39

2.4. Standard gradings of classical Lie algebras 42

2.5. The Lie algebras of Cartan type 44

2.6. The Jacobson-Witt algebras 45

2.7. Divided power algebras $\quad 46$

2.8. Witt Lie algebras of Cartan type (the $\boldsymbol{W}$ series) 47

2.9. Special Lie algebras of Cartan type (the $\boldsymbol{S}$ series) 49

2.10. Hamiltonian Lie algebras of Cartan type (the $\boldsymbol{H}$ series) 51

2.11. Contact Lie algebras of Cartan type (the $\boldsymbol{K}$ series) 55

2.12. The Recognition Theorem with stronger hypotheses 58

2.13. $\mathfrak{g}_{\ell}$ as a $\mathfrak{g}_{0}$-module for Lie algebras $\mathfrak{g}$ of Cartan type $\quad 59$

2.14. Melikyan Lie algebras 67 
Chapter 3. The Contragredient Case $\quad 71$

3.1. Introduction 71

3.2. Results on modules for three-dimensional Lie algebras $\quad 71$

3.3. Primitive vectors in $\mathfrak{g}_{\mathbf{1}}$ and $\mathfrak{g}_{-\mathbf{1}} \quad 76$

$\begin{array}{ll}\text { 3.4. Subalgebras with a balanced grading } & 79\end{array}$

$\begin{array}{lll}\text { 3.5. Algebras with an unbalanced grading } & 87\end{array}$

$\begin{array}{lll}\text { Chapter 4. The Noncontragredient Case } & 97\end{array}$

4.1. General assumptions and notation $\quad 97$

4.2. Brackets of weight vectors in opposite gradation spaces $\quad 98$

4.3. Determining $\mathfrak{g}_{\mathbf{0}}$ and its representation on $\mathfrak{g}_{-\mathbf{1}} \quad 99$

4.4. Additional assumptions 105

4.5. Computing weights of $\mathfrak{b}^{-}$-primitive vectors in $\mathfrak{g}_{1}$

4.6. Determination of the local Lie algebra 115

4.7. The irreducibility of $\mathfrak{g}_{1} \quad 125$

4.8. Determining the negative part when $\mathfrak{g}_{\mathbf{1}}$ is irreducible 133

4.9. Determining the negative part when $\mathfrak{g}_{1}$ is reducible 137

4.10. The case that $\mathfrak{g}_{\mathbf{0}}$ is abelian 141

4.11. Completion of the proof of the Main Theorem 142

$\begin{array}{ll}\text { Bibliography } & 143\end{array}$ 


\begin{abstract}
The "Recognition Theorem" for graded Lie algebras is an essential ingredient in the classification of finite-dimensional simple Lie algebras over an algebraically closed field of characteristic $p>3$. The main goal of this monograph is to present the first complete proof of this fundamental result.
\end{abstract}

Received by the editor November 23, 2018.

1991 Mathematics Subject Classification. Primary 17B50, 17B70, 17B20 Secondary $17 \mathrm{~B} 05$.

Key words and phrases. graded Lie algebras; simple Lie algebras of prime characteristic; classical, Cartan type, and Melikyan Lie algebras.

*The first author gratefully acknowledges support from National Science Foundation grant \#DMS-0245082 and from the E.B. Van Vleck Professorship at the University of Wisconsin-Madison. 



\section{Introduction}

The focus of this work is the following Main Theorem, often referred to as the "Recognition Theorem," because of its extensive use in recognizing certain graded Lie algebras from their null components.

THEOREM 0.1. Let $\mathfrak{g}=\bigoplus_{j=-q}^{r} \mathfrak{g}_{j}$ be a finite-dimensional graded Lie algebra over an algebraically closed field $\mathbb{F}$ of characteristic $p>3$. Assume that:

(a) $\mathfrak{g}_{0}$ is a direct sum of ideals, each of which is abelian, a classical simple Lie algebra, or one of the Lie algebras $\mathfrak{g l}_{n}, \mathfrak{s l}_{n}$, or $\mathfrak{p g l}_{n}$ with $p \mid n$;

(b) $\mathfrak{g}_{-1}$ is an irreducible $\mathfrak{g}_{0}$-module;

(c) If $x \in \bigoplus_{j \geq 0} \mathfrak{g}_{j}$ and $\left[x, \mathfrak{g}_{-1}\right]=0$, then $x=0$;

(d) If $x \in \bigoplus_{j \geq 0} \mathfrak{g}_{-j}$ and $\left[x, \mathfrak{g}_{1}\right]=0$, then $x=0$.

Then $\mathfrak{g}$ is isomorphic as a graded Lie algebra to one of the following:

(1) a classical simple Lie algebra with a standard grading;

(2) $\mathfrak{p g l}_{m}$ for some $m$ such that $p \mid m$ with a standard grading;

(3) a Cartan type Lie algebra with the natural grading or its reverse;

(4) a Melikyan ${ }^{1}$ algebra (in characteristic 5) with either the natural grading or its reverse.

The classical simple Lie algebras in this theorem are the algebras obtained by reduction modulo $p$ of the finite-dimensional complex simple Lie algebras, as described in [S, Sec. 10] (see also Section 2.2). Thus, they are of type $\mathrm{A}_{n-1}, p \nmid n, \mathrm{~B}_{n}, \mathrm{C}_{n}, \mathrm{D}_{n}, \mathrm{E}_{6}, \mathrm{E}_{7}, \mathrm{E}_{8}, \mathrm{~F}_{4}, \mathrm{G}_{2}$, or they are isomorphic to $\mathfrak{p s l}_{n}$ where $p \mid n$.

The Recognition Theorem is an essential ingredient in the classification of the finite-dimensional simple Lie algebras over algebraically closed fields of characteristic $p>3$. In a sense, the whole classification theory is built around this theorem, as the theory aims to show that any finite-dimensional simple Lie algebra $L$ admits a filtration $L=L_{-q} \supset \ldots \supset L_{0} \supset \ldots \supset L_{r} \supset$ $L_{r+1}=0$ such that the corresponding graded Lie algebra $\mathfrak{g}=\bigoplus_{i=-q}^{r} \mathfrak{g}_{i}$, where $\mathfrak{g}_{i}=L_{i} / L_{i+1}$, satisfies conditions (a)-(d) above. The Recognition Theorem is used several times throughout the classification; its first application results in a complete list of the simple Lie algebras of absolute toral

\footnotetext{
$1_{\text {transliterated as Melikian in many references such as }}[\mathbf{S t}]$, for example
} 
rank two, and its last application yields a crucial characterization of the Melikyan algebras, thereby completing the classification. The main goal of this monograph is to present the first complete proof of this fundamental theorem.

V.G. Kac first undertook to prove the Recognition Theorem in [K2]. This pioneering work was ahead of its time. In 1970, very little was known about rational representations of simple algebraic groups in prime characteristic, and the Melikyan algebras were discovered only in the 1980s. Despite that, Kac made many deep and important observations towards the proof of the theorem in [K2]. Most of them are incorporated in Chapters 3 and 4 of this monograph.

Historical accounts of the classification of simple Lie algebras of characteristic $p>0$ can be found for example in $[\mathbf{M}]$, $[\mathbf{W i 3}]$, and $[\mathbf{B}]$. Investigation of the finite-dimensional simple Lie algebras over algebraically closed fields of positive characteristic began in the 1930s in the work of Jacobson, Witt, and Zassenhaus. During the next quarter century, many examples of such Lie algebras were discovered. In [S], written in 1967, Seligman spoke of a "rather awkward array of simple modular Lie algebras which would be totally unexpected to one acquainted only with the non-modular case." Seligman's book contained a characterization of the classical Lie algebras of characteristic $p>3$; that is, those obtained from $\mathbb{Z}$-forms of the finite-dimensional simple Lie algebras over $\mathbb{C}$ by reducing modulo $p$. (See Section 2.2.) It was about the same time that Kostrikin and Šafarevič $[\mathbf{K S}]$ observed a similarity between the known nonclassical simple Lie algebras of prime characteristic and the four families $W, S, H, K$ (Witt, special, Hamiltonian, contact) of infinite-dimensional complex Lie algebras arising in Cartan's work on Lie pseudogroups. They called their analogues "Lie algebras of Cartan type" and formulated a conjecture which shaped research on the subject during the next thirty years. The Kostrikin-Šafarevič Conjecture of 1966 states

Over an algebraically closed field of characteristic $p>5$, a finitedimensional restricted simple Lie algebra is classical or of Cartan type.

In 1984, Block and Wilson [BW] succeeded in proving this conjecture for algebraically closed fields of characteristic $p>7$.

If the notion of a Cartan type Lie algebra is expanded to include both the restricted and nonrestricted ones as well as their filtered deformations (determined later by Kac [K3], Wilson [Wi2], and Skryabin [Sk1]), then one can formulate the Generalized Kostrikin-Safarevič Conjecture by simply erasing the word restricted in the statement above.

The Generalized Kostrikin-Šafarevič Conjecture is now a theorem for $p>7$. First announced by Strade and Wilson $[\mathbf{S W}]$ in 1991, its proof is spread over a number of papers. As mentioned above, the proof depends in a critical way on the above Recognition Theorem (hence on our monograph). 
We refer the interested reader to $[\mathbf{S t}]$ for a comprehensive exposition of the classification.

Recent work of Strade and the third author [PS1]-[PS5] has made significant progress on the problem of classifying the finite-dimensional simple Lie algebras over fields of characteristic 7 and of characteristic 5, where the Generalized Kostrikin-Šafarevič Conjecture is known to fail because of the Melikyan algebras. (See Section 2.45.) The Classification Theorem announced in [St, p. 7] (and also in [P2]), asserts "Every finite dimensional simple Lie algebra over an algebraically closed field of characteristic $p>3$ is of classical, Cartan or Melikyan type." The Recognition Theorem plays a vital role in this extension of the classification theory to $p=5$ and 7 . In characteristics 2 and 3, many more simple Lie algebras which are neither classical nor Cartan type are known (characteristic 3 examples can be found in $[\mathbf{S t}$, Sec. 4.4]). The papers $[\mathbf{K O}],[\mathbf{B K K}],[\mathbf{B G K}]$, and $[\mathbf{G K}]$ prove recognition theorems for graded Lie algebras of characteristic 3 under various assumptions on the gradation spaces. One of the main challenges in characteristics 2 and 3 will be to remove such extra assumptions and determine all finite-dimensional graded Lie algebras $\mathfrak{g}$ satisfying conditions (b), (c), (d) above with the graded component $\mathfrak{g}_{0}$ isomorphic to the Lie algebra of a reductive group. Once this is accomplished, one might be able to formulate a plausible analogue of the Generalized Kostrikin-Šafarevič Conjecture for $p=2$ and 3 and to begin the classification work in a systematic way.

Our monograph consists of four chapters. In the first, we establish general properties of graded Lie algebras and use them to show that in a finite-dimensional graded Lie algebra satisfying conditions (a)-(d) of the

Main Theorem, the representation of the commutator ideal $\mathfrak{g}_{0}^{(1)}$ of the null component $\mathfrak{g}_{0}$ on $\mathfrak{g}_{-1}$ must be restricted. In Chapter 2, we gather useful information about known graded Lie algebras, both classical and nonclassical. Chapter 3 deals with the case in which $\mathfrak{g}_{-1}$ and $\mathfrak{g}_{1}$ are dual $\mathfrak{g}_{0}$-modules, the so-called contragredient case, which leads to the classical or Melikyan Lie algebras. Chapter 4 treats the noncontragredient case, and there the graded Lie algebras are shown to be of Cartan type.

\section{Acknowledgments}

Work on this monograph began under the sponsorship of National Science Foundation US-FSU Cooperative Program Grant \#DMS-9115984, which funded the visits of Alexei Kostrikin, Michael Kuznetsov, and Alexander Premet to the University of Wisconsin-Madison. We are grateful for the Foundation's support and for the enthusiasm that Alexei Kostrikin showed in urging us to undertake this project. We regret that he did not live to see its completion. We also wish to express our sincere thanks to Richard Block, Michael Kuznetsov, Hayk Melikyan, Yuri Razmyslov, George Seligman, Serge Skryabin, Helmut Strade, and Robert Wilson for their interest and encouragement. 


\section{CHAPTER 1}

\section{Graded Lie Algebras}

\subsection{Introduction}

In this chapter, we develop results about general graded Lie algebras. Later (starting in Section 1.8) and in subsequent chapters, we specialize to modular graded Lie algebras satisfying the hypotheses of the Recognition Theorem.

To begin, our focus is on Lie algebras over an arbitrary field $\mathbb{F}$ having an integer grading,

$$
\mathfrak{g}=\bigoplus_{i=-q}^{r} \mathfrak{g}_{i},
$$

where $\left[\mathfrak{g}_{i}, \mathfrak{g}_{j}\right] \subseteq \mathfrak{g}_{i+j}$ if $-q \leq i+j \leq r$ and $\left[\mathfrak{g}_{i}, \mathfrak{g}_{j}\right]=0$ otherwise. Then $\mathfrak{g}_{0}$ is a subalgebra of $\mathfrak{g}$, and each subspace $\mathfrak{g}_{j}$ is a $\mathfrak{g}_{0}$-module under the adjoint action. The spaces

$$
\mathfrak{g}_{\leq 0}:=\mathfrak{g}_{-} \oplus \mathfrak{g}_{0} \quad \text { and } \quad \mathfrak{g}_{\geq 0}:=\mathfrak{g}_{0} \oplus \mathfrak{g}_{+}
$$

are also subalgebras of $\mathfrak{g}$, where

$$
\mathfrak{g}_{-}:=\bigoplus_{i=1}^{q} \mathfrak{g}_{-i} \quad \text { and } \quad \mathfrak{g}_{+}:=\bigoplus_{j=1}^{r} \mathfrak{g}_{j}
$$

are nilpotent ideals of $\mathfrak{g}_{\leq 0}$ and $\mathfrak{g}_{\geq 0}$ respectively. If $\mathfrak{g}_{-q}$ and $\mathfrak{g}_{r}$ are nonzero, then $q$ is said to be the depth and $r$ the height of $\mathfrak{g}$. We assume that $q, r \geq 1$ and $q$ is finite, but in this section and the next allow the possibility that the height $r$ is infinite. The following conditions play a key role in this investigation:

$$
\mathfrak{g}_{-1} \text { is an irreducible } \mathfrak{g}_{0} \text {-module. }
$$

$$
\text { If } x \in \mathfrak{g}_{\geq 0} \text { and }\left[x, \mathfrak{g}_{-1}\right]=0 \text {, then } \quad x=0 .
$$

Property (1.1) is termed irreducibility and (1.2) is called transitivity. When we say an algebra is irreducible and transitive, we mean that both (1.1) and (1.2) hold. On occasion we refer to algebras satisfying the following constraint as being 1-transitive, or having 1-transitivity: 


$$
\text { If } x \in \mathfrak{g}_{\leq 0} \text { and }\left[x, \mathfrak{g}_{1}\right]=0 \text {, then } \quad x=0 .
$$

\subsection{The Weisfeiler radical}

Every graded Lie algebra $\mathfrak{g}$ has a radical, which was first introduced by Weisfeiler [W], and which is constructed as follows: $\quad \operatorname{Set} \mathcal{M}^{0}(\mathfrak{g})=0$, and for $i \geq 0$ define $\mathcal{M}^{i+1}(\mathfrak{g})$ inductively by

$$
\mathcal{M}^{i+1}(\mathfrak{g})=\left\{x \in \mathfrak{g}_{-} \mid\left[x, \mathfrak{g}_{+}\right] \subseteq \mathcal{M}^{i}(\mathfrak{g})\right\}
$$

Then

$$
\begin{aligned}
0=\mathcal{M}^{0}(\mathfrak{g}) \subseteq \mathcal{M}^{1}(\mathfrak{g}) \subseteq \cdots & \subseteq \mathcal{M}^{i-1}(\mathfrak{g}) \subseteq \mathcal{M}^{i}(\mathfrak{g}) \subseteq \ldots \text { and } \\
\mathcal{M}(\mathfrak{g}) & :=\bigcup_{i} \mathcal{M}^{i}(\mathfrak{g})
\end{aligned}
$$

is called the Weisfeiler radical of $\mathfrak{g}$. By its definition, $\mathcal{M}(\mathfrak{g})$ is a subspace of $\mathfrak{g}_{-}$invariant under bracketing by $\mathfrak{g}_{+}$, and for $j=0,1, \ldots, q$,

$$
\begin{aligned}
{\left[\left[\mathcal{M}^{i}(\mathfrak{g}), \mathfrak{g}_{-j}\right], \mathfrak{g}_{+}\right] } & \subseteq\left[\left[\mathcal{M}^{i}(\mathfrak{g}), \mathfrak{g}_{+}\right], \mathfrak{g}_{-j}\right]+\left[\mathcal{M}^{i}(\mathfrak{g}),\left[\mathfrak{g}_{-j}, \mathfrak{g}_{+}\right]\right] \\
& \subseteq\left[\mathcal{M}^{i-1}(\mathfrak{g}), \mathfrak{g}_{-j}\right]+\left[\mathcal{M}^{i}(\mathfrak{g}), \sum_{\ell \geq 1} \mathfrak{g}_{-j+\ell}\right]
\end{aligned}
$$

Now when $j=0$ and $i=1$, the right side of (1.6) is zero, which implies that $\left[\mathcal{M}^{1}(\mathfrak{g}), \mathfrak{g}_{0}\right] \subseteq \mathcal{M}^{1}(\mathfrak{g})$. We may assume that $\left[\mathcal{M}^{i-1}(\mathfrak{g}), \mathfrak{g}_{0}\right] \subseteq \mathcal{M}^{i-1}(\mathfrak{g})$. Then (1.6) shows that $\left[\mathcal{M}^{i}(\mathfrak{g}), \mathfrak{g}_{0}\right] \subseteq \mathcal{M}^{i}(\mathfrak{g})$. Suppose we know that

$$
\left[\mathcal{M}^{i}(\mathfrak{g}), \mathfrak{g}_{-k}\right] \subseteq \mathcal{M}^{i+k}(\mathfrak{g})
$$

for all $0 \leq k<j$. Then by (1.6) and induction on $i$ we have,

$$
\left[\left[\mathcal{M}^{i}(\mathfrak{g}), \mathfrak{g}_{-j}\right], \mathfrak{g}_{+}\right] \subseteq\left[\mathcal{M}^{i-1}(\mathfrak{g}), \mathfrak{g}_{-j}\right]+\left[\mathcal{M}^{i}(\mathfrak{g}), \sum_{\ell \geq 1} \mathfrak{g}_{-j+\ell}\right] \subseteq \mathcal{M}^{i+j-1}(\mathfrak{g})
$$

Consequently, $\left[\mathcal{M}^{i}(\mathfrak{g}), \mathfrak{g}_{-j}\right] \subseteq \mathcal{M}^{i+j}(\mathfrak{g})$ for $j=0,1, \ldots$ Thus $\mathcal{M}(\mathfrak{g})$ is an ideal of $\mathfrak{g}$, and it exhibits the following characteristics enjoyed by a "radical":

Proposition 1.8 .

(i) $\mathcal{M}(\mathfrak{g})$ is a graded ideal of $\mathfrak{g}$ contained in $\mathfrak{g}_{-}$.

(ii) Suppose that $\mathfrak{g}$ is irreducible (1.1) and transitive (1.2), and let $J$ be an ideal of $\mathfrak{g}$ contained in $\mathfrak{g}_{-}$. Then $J \subseteq \mathcal{M}(\mathfrak{g}) \subseteq \sum_{i \geq 2} \mathfrak{g}_{-i}$. Thus, $\mathcal{M}(\mathfrak{g})$ is the sum of all the ideals of $\mathfrak{g}$ contained in $\mathfrak{g}_{-}$. Moreover, $\mathfrak{g} / \mathcal{M}(\mathfrak{g})$ is irreducible and transitive, and $\mathcal{M}(\mathfrak{g} / \mathcal{M}(\mathfrak{g}))=0$. 
(iii) If $\mathfrak{g}$ is irreducible and transitive, and $\left[\mathfrak{g}_{-i}, \mathfrak{g}_{-1}\right]=\mathfrak{g}_{-(i+1)}$ for all $i \geq 1$, then for any ideal $J$ of $\mathfrak{g}$ either $J \subseteq \mathcal{M}(\mathfrak{g})$ or $J \supseteq \mathfrak{g}_{-}$.

(iv) If $\mathfrak{g}$ is 1-transitive (1.3), then $\mathcal{M}(\mathfrak{g})=0$.

Proof. (i) We know from the calculations above that $\mathcal{M}(\mathfrak{g})$ is an ideal of $\mathfrak{g}$. If $x=x_{-q}+\cdots+x_{-1} \in \mathcal{M}(\mathfrak{g})$, then one can see by bracketing with homogeneous elements of positive degree that the homogeneous components $x_{-i} \in \mathfrak{g}_{-i}$ of $x$ belong to $\mathcal{M}(\mathfrak{g})$ also, and the quotient algebra $\overline{\mathfrak{g}}:=\mathfrak{g} / \mathcal{M}(\mathfrak{g})$ is graded.

(ii) Suppose that $\mathfrak{g}$ is irreducible and transitive. If $J$ is any ideal of $\mathfrak{g}$ contained in $\mathfrak{g}_{-}$and if $J \cap \mathfrak{g}_{-1} \neq 0$, then $J \cap \mathfrak{g}_{-1}=\mathfrak{g}_{-1}$ by irreducibility, since $J \cap \mathfrak{g}_{-1}$ is a $\mathfrak{g}_{0}$-submodule of $\mathfrak{g}_{-1}$. But if $J \supseteq \mathfrak{g}_{-1}$, then $J \supseteq\left[\mathfrak{g}_{-1}, \mathfrak{g}_{1}\right] \neq 0$ by transitivity, to contradict $J \subseteq \mathfrak{g}_{-}$. Hence, when $\mathfrak{g}$ is irreducible and transitive, every ideal $J$ of $\mathfrak{g}$ contained in $\mathfrak{g}_{-}$has trivial intersection with $\mathfrak{g}_{-1}$. In particular, since $\mathcal{M}(\mathfrak{g}) \subseteq \mathfrak{g}_{\text {- }}$ and $\mathcal{M}(\mathfrak{g})$ is graded, we must have $\mathcal{M}(\mathfrak{g}) \subseteq$ $\sum_{i>2} \mathfrak{g}_{-i}$. It follows that $\mathfrak{g} / \mathcal{M}(\mathfrak{g})$ will inherit the properties of irreducibility and transitivity.

Now suppose that $J$ is an ideal of $\mathfrak{g}$ contained in $\mathfrak{g}_{-}$and let $x_{-q}+\cdots+$ $x_{-1} \in J$, where $x_{-i} \in \mathfrak{g}_{-i}$ for all $i$. Then it must be that $x_{-i} \in \mathcal{M}^{i}(\mathfrak{g})$ for $i=1, \ldots, q$. Consequently $J \subseteq \mathcal{M}(\mathfrak{g}) \subseteq \sum_{i \geq 2} \mathfrak{g}_{-i}$, and $\mathcal{M}(\mathfrak{g})$ is the sum of all the ideals of $\mathfrak{g}$ contained in $\mathfrak{g}$. . Any ideal of $\overline{\mathfrak{g}}=\mathfrak{g} / \mathcal{M}(\mathfrak{g})$ contained in $\overline{\mathfrak{g}}_{-}$has the form $\bar{K}$ where $K$ is an ideal of $\mathfrak{g}$ contained in $\mathfrak{g}_{-}$. But then $K \subseteq \mathcal{M}(\mathfrak{g})$ so $\bar{K}=0$. Hence, $\mathcal{M}(\overline{\mathfrak{g}})=0$.

(iii) Under the hypotheses in (iii), suppose that $J$ is an ideal of $\mathfrak{g}$, and define the following subspaces of $\mathfrak{g}$ :

$$
Y_{j}:=\left\{\begin{array}{l|l}
\left.y \in \mathfrak{g}_{j} \mid y+z \in J \text { for some } z \in \sum_{i \leq j-1} \mathfrak{g}_{i}\right\} .
\end{array}\right.
$$

Then $Y_{j}$ is a $\mathfrak{g}_{0}$-submodule of $\mathfrak{g}_{j}$ for each $j$. Now either $J \subseteq \mathfrak{g}_{-}$(and hence $J \subseteq \mathcal{M}(\mathfrak{g})$ by (ii)) or there exists an element $x=x_{-q}+\cdots+x_{k} \in J$ with $x_{k} \neq 0$ and $k \geq 0$. Since in the second case $\left(\operatorname{ad} \mathfrak{g}_{-1}\right)^{k+1}(x) \neq 0$ by the transitivity of $\mathfrak{g}$, we see that $J \cap \mathfrak{g}_{-} \neq 0$ and $Y_{-1} \neq 0$. Hence by irreducibility, $Y_{-1}$ must equal $\mathfrak{g}_{-1}$. Assume we have shown that $Y_{-i}=\mathfrak{g}_{-i}$. Then $Y_{-(i+1)} \supseteq\left[Y_{-i}, \mathfrak{g}_{-1}\right]=\left[\mathfrak{g}_{-i}, \mathfrak{g}_{-1}\right]=\mathfrak{g}_{-(i+1)}$ so that $Y_{-(i+1)}=\mathfrak{g}_{-(i+1)}$. In particular, $J \supseteq \mathfrak{g}_{-q}$. Suppose that $J \supseteq \mathfrak{g}_{-t}$ for all $t$ with $i+1 \leq t \leq q$. Since $Y_{-i}=\mathfrak{g}_{-i}$, it follows that $J \supseteq \mathfrak{g}_{-i}$. Consequently $J \supseteq \mathfrak{g}_{-}$, as asserted.

(iv) Suppose $\mathcal{M}^{k}(\mathfrak{g})=0$ for $0 \leq k<i$. Then $\left[\mathcal{M}^{i}(\mathfrak{g}), \mathfrak{g}_{1}\right] \subseteq \mathcal{M}^{i-1}(\mathfrak{g})=0$. Thus, if $\mathfrak{g}$ is 1 -transitive, we must have $\mathcal{M}^{i}(\mathfrak{g})=0$ also. Therefore, 1transitivity implies $\mathcal{M}(\mathfrak{g})=\bigcup \mathcal{M}^{i}(\mathfrak{g})=0$. 
Proposition 1.10. Assume $\mathfrak{g}=\bigoplus_{i=-q}^{r} \mathfrak{g}_{i}$ is an irreducible, transitive graded Lie algebra such that $\mathfrak{g}_{-i}=\left[\mathfrak{g}_{-1}, \mathfrak{g}_{-i+1}\right]$ for all $i \geq 2$. If $\mathcal{M}(\mathfrak{g})=0$, then $\mathfrak{g}$ contains no abelian ideals.

Proof. By Proposition 1.8 (iii), any nonzero ideal $J$ of $\mathfrak{g}$ must contain $\mathfrak{g}_{-}$and hence must contain $\mathfrak{g}_{-1} \oplus\left[\mathfrak{g}_{-1}, \mathfrak{g}_{1}\right]$. By transitivity $(1.2),[J, J] \supseteq$ $\left[\mathfrak{g}_{-1},\left[\mathfrak{g}_{-1}, \mathfrak{g}_{1}\right]\right] \neq 0$. Consequently, $J$ cannot be abelian.

Corollary 1.11. If $\mathfrak{g}=\bigoplus_{i=-q}^{r} \mathfrak{g}_{i}$ is a irreducible, transitive graded Lie algebra such that $\mathfrak{g}_{-i}=\left[\mathfrak{g}_{-1}, \mathfrak{g}_{-i+1}\right]$ for all $i \geq 2$, then $\mathfrak{g} / \mathcal{M}(\mathfrak{g})$ is semisimple.

Proof. This is an immediate consequence of Proposition 1.10 and the fact that $\mathcal{M}(\overline{\mathfrak{g}})=0$ for $\overline{\mathfrak{g}}=\mathfrak{g} / \mathcal{M}(\mathfrak{g})$ by (iii) of Proposition 1.8 .

Lemma 1.12. Assume $\mathfrak{g}=\bigoplus_{i=-q}^{r} \mathfrak{g}_{i}$ is a graded Lie algebra such that $\mathfrak{g}_{+}$is generated by $\mathfrak{g}_{1}$ and $\mathcal{M}(\mathfrak{g})=0$. If $x \in \mathfrak{g}_{-}$and $\left[x, \mathfrak{g}_{1}\right]=0$, then $x=0$.

Proof. The hypotheses imply that $\left[x, \mathfrak{g}_{+}\right]=0$, so that $x \in \mathcal{M}^{1}(\mathfrak{g})=0$.

\subsection{The minimal ideal $\mathfrak{J}$}

In this section we show that graded Lie algebras satisfying certain conditions must contain a unique minimal ideal $\mathcal{J}$ which is graded, and we derive some properties of $\mathcal{J}$.

Proposition 1.13. (Compare [W], Prop. 1.61, Cor. 1.62, Cor. 1.66.) Assume $\mathfrak{g}=\bigoplus_{i=-q}^{r} \mathfrak{g}_{i}$ is an irreducible, transitive graded Lie algebra such that $\mathfrak{g}_{-i}=\left[\mathfrak{g}_{-1}, \mathfrak{g}_{-i+1}\right]$ for all $i \geq 2$. If $\mathcal{M}(\mathfrak{g})=0$, then $\mathfrak{g}$ has a unique minimal ideal $\mathfrak{J}$ which is graded and contains $\mathfrak{g}_{-}$.

Proof. Since $\mathcal{M}(\mathfrak{g})=0$, every nonzero ideal must contain $\mathfrak{g}_{\text {- by Proposition }}$ 1.8 (iii). Therefore the intersection $\mathcal{J}$ of all the ideals is the unique minimal ideal of $\mathfrak{g}$, and it contains $\mathfrak{g}_{-}$. Let $\mathcal{J}_{j}:=\mathcal{J} \cap \mathfrak{g}_{j}$ for each $j$, and observe that $\mathcal{J}_{-i}=\mathfrak{g}_{-i}$ for all $i \geq 1$. The space $\mathcal{J}^{\prime}=\bigoplus_{j} \mathcal{J}_{j}$ is a nonzero ideal of $\mathfrak{g}$, since $\left[\mathfrak{g}_{i}, \mathcal{J}_{j}\right] \subseteq \mathfrak{g}_{i+j} \cap \mathcal{J}=\mathcal{J}_{i+j}$. By the minimality of $\mathcal{J}$, we conclude that $\mathcal{J}=\bigoplus_{j} \mathcal{J}_{j}$.

Corollary 1.14. Assume $\mathfrak{g}=\bigoplus_{i=-q}^{r} \mathfrak{g}_{i}$ is an irreducible, transitive graded Lie algebra such that $\mathfrak{g}_{-i}=\left[\mathfrak{g}_{-1}, \mathfrak{g}_{-i+1}\right]$ for all $i \geq 2$. Then $\overline{\mathfrak{g}}=$ $\mathfrak{g} / \mathcal{M}(\mathfrak{g})$ has a unique minimal ideal which is graded and contains $\overline{\mathfrak{g}}_{-}$. 
From now on, we assume that the height $r$ of the graded Lie algebras is finite.

Assume $\mathfrak{g}$ is an irreducible, transitive graded Lie algebra with $\mathcal{M}(\mathfrak{g})=0$ and with $\mathfrak{g}_{-}$generated by $\mathfrak{g}_{-1}$, and let $\mathcal{J}$ be the unique minimal ideal of $\mathfrak{g}$ from Proposition 1.13. Then

$$
\mathcal{R}:=\sum_{k \geq 1}\left(\operatorname{ad} \mathfrak{g}_{-1}\right)^{k} \mathfrak{g}_{r} \subseteq \mathcal{J}
$$

is stable under the action of both ad $\mathfrak{g}_{-1}$ and ad $\mathfrak{g}_{0}$. By the Jacobi identity, $\left[\mathfrak{g}_{+}, \mathcal{R}\right] \subseteq \mathcal{R}+\mathfrak{g}_{r}$. As $\mathfrak{g}_{-}$is generated by $\mathfrak{g}_{-1}$ by assumption, $\mathcal{R}+\mathfrak{g}_{r}$ is a graded ideal of $\mathfrak{g}$. It follows that $\mathcal{J} \subseteq \mathcal{R}+\mathfrak{g}_{r}$. Moreover,

$$
\mathcal{J}=\mathcal{J} \cap\left(\mathcal{R}+\mathfrak{g}_{r}\right)=\mathcal{R}+\left(\mathcal{J} \cap \mathfrak{g}_{r}\right) \subseteq \mathcal{J}
$$

so $\mathcal{J}=\mathcal{R}+\left(\mathcal{J} \cap \mathfrak{g}_{r}\right)$. This establishes the following result:

Lemma 1.16. Any transitive, irreducible graded Lie algebra $\mathfrak{g}$ with $\mathcal{M}(\mathfrak{g})$ $=0$ and $\mathfrak{g}_{-}$generated by $\mathfrak{g}_{-1}$ contains a unique minimal graded ideal $\mathcal{J}$ such that

$$
\mathcal{J}=\sum_{k \geq 1}\left(\operatorname{ad} \mathfrak{g}_{-1}\right)^{k} \mathfrak{g}_{r}+\left(\mathcal{J} \cap \mathfrak{g}_{r}\right) .
$$

Thus, $\mathcal{J}=\bigoplus_{i=-q}^{s} \mathcal{J}_{i}$ where $\mathcal{J}_{s} \neq 0$, and $s=r$ if $\mathcal{J} \cap \mathfrak{g}_{r} \neq 0$, and $s=r-1$ otherwise. Moreover,

$$
\mathcal{J}_{i}= \begin{cases}\left(\operatorname{ad~} \mathfrak{g}_{-1}\right)^{r-i} \mathfrak{g}_{r} & \text { if } 0 \leq i \leq r-1, \\ \mathfrak{g}_{i} & \text { if }-q \leq i \leq-1\end{cases}
$$

\subsection{The graded algebras $\mathcal{B}\left(V_{-t}\right)$ and $\mathcal{B}\left(V_{t}\right)$}

There is a general procedure described by Benkart and Gregory in $[\mathbf{B G}$, Sec. 3] for producing quotients of subalgebras in a graded Lie algebra $\mathfrak{g}=$ $\bigoplus_{i=-q}^{r} \mathfrak{g}_{i}$. It has been applied subsequently in several other settings; for example, it was used by Skryabin in $[\mathbf{S k 2}]$ to derive information about the solvable radical of $\mathfrak{g}_{0}$.

The procedure begins with a subalgebra $F_{0}$ of $\mathfrak{g}_{0}$ and an $F_{0}$-submodule $F_{-1}$ of $\mathfrak{g}_{-t}$ for some $t$ with $1 \leq t \leq q$. For $i>0$ define:

$$
\begin{aligned}
& F_{-i}=\left[F_{-1}, F_{-i+1}\right] \\
& F_{i}=\left\{y \in \mathfrak{g}_{i t} \mid\left[y, F_{-1}\right] \subseteq F_{i-1}\right\}
\end{aligned}
$$


We claim that $F=\bigoplus_{j} F_{j}$ is a subalgebra of $\mathfrak{g}$. To see this, note that $\left[F_{-1}, F_{j}\right] \subseteq F_{j-1}$ for all $j$. Assume for $1 \leq i<k$ that $\left[F_{-i}, F_{j}\right] \subseteq F_{j-i}$ for all $j$. Then

$$
\begin{aligned}
{\left[F_{-k}, F_{j}\right]=\left[\left[F_{-1}, F_{-k+1}\right], F_{j}\right] } & \subseteq\left[\left[F_{-1}, F_{j}\right], F_{-k+1}\right]+\left[F_{-1},\left[F_{-k+1}, F_{j}\right]\right] \\
& \subseteq F_{j-k}
\end{aligned}
$$

for all $j$. We show that $\left[F_{i}, F_{j}\right] \subseteq F_{i+j}$ for $i, j \geq 0$ by induction on $i+j$. If $i+j=0$, the result is clear, so we may assume that $i+j>0$ and that the result holds for values $<i+j$. Then $\left[F_{-1},\left[F_{i}, F_{j}\right]\right] \subseteq\left[F_{i-1}, F_{j}\right]+\left[F_{i}, F_{j-1}\right]$. Now if $i-1$ or $j-1$ is negative, we may use what has been established previously. Otherwise, we may apply the inductive hypothesis. In either event, the sum on the right is in $F_{i+j-1}$, and $\left[F_{i}, F_{j}\right] \subseteq F_{i+j}$ by definition. To summarize, we have shown:

Proposition 1.19. Let $\mathfrak{g}$ be an arbitrary graded Lie algebra. Let $F_{0}$ be a subalgebra of $\mathfrak{g}_{0}$, and for $t>0$ let $F_{-1}$ be an $F_{0}$-submodule of $\mathfrak{g}_{-t}$. If $F_{-i}$ and $F_{i}$ are defined by (1.17) and (1.18), then $F=\bigoplus_{j} F_{j}$ is a graded subalgebra of $\mathfrak{g}$.

Proposition 1.20. Let $F=\bigoplus_{j} F_{j}$ be any graded Lie algebra satisfying (1.17), and assume that $F_{-1}$ is a nontrivial $F_{0}$-module under the adjoint action. Set $A_{-i}=0$ for all $i>0$. For $i \geq 0$, define $A_{i}=\left\{y \in F_{i} \mid\left[y, F_{-1}\right] \subseteq\right.$ $\left.A_{i-1}\right\}$. Then $A=\bigoplus_{j} A_{j}$ is a graded ideal of $F$. Moreover the graded Lie algebra $\mathcal{B}=\bigoplus_{i} \mathcal{B}_{i}=F / A$ satisfies (1.2) (transitivity) and (1.17).

Proof. Assume $F$ is any graded Lie algebra satisfying the assumptions and $A_{j}$ is as in the statement of the proposition. Then $\left[F_{-1}, A\right] \subseteq A$, and it follows by induction and the Jacobi identity that $\left[F_{-i}, A\right] \subseteq A$ for all $i>0$. We suppose that $i, j \geq 0$ and prove that $\left[F_{i}, A_{j}\right] \subseteq A$ for all such $i$ and $j$ by induction on $i+j$. The result is clear if $i+j=0$, for the annhilator of $F_{-1}$ is an ideal of $F_{0}$. Now $\left[F_{-1},\left[F_{i}, A_{j}\right]\right] \subseteq\left[F_{i-1}, A_{j}\right]+\left[F_{i}, A_{j-1}\right] \subseteq A_{j+i-1}$ by induction. Thus, $\left[F_{i}, A_{j}\right] \subseteq A_{i+j}$, and $A$ is an ideal as desired.

That (1.17) holds in $\mathcal{B}=F / A$ is obvious since $A_{j}=0$ for $j<0$. Suppose for $\bar{y} \in \mathcal{B}_{j}$ with $j \geq 0$ that $\left[\bar{y}, \mathcal{B}_{-1}\right]=0$. If $\bar{y}=y+A_{j}$, then $\left[y, F_{-1}\right] \subseteq A_{j-1}$, and that implies $y \in A_{j}$ so that $\bar{y}=0$. Thus, $\mathcal{B}$ is transitive.

We note that in this construction, $A_{0}=\operatorname{Ann}_{F_{0}}\left(F_{-1}\right)$, the annihilator of $F_{-1}$ in $F_{0}$.

By symmetry we have:

Proposition 1.21. Let $\mathfrak{g}$ be an arbitrary graded Lie algebra. Let $F_{0}$ be a subalgebra of $\mathfrak{g}_{0}$, and let $F_{1}$ be an $F_{0}$-submodule of $\mathfrak{g}_{t}$ for some $t \geq 1$. For $i>0$, define $F_{i}$ and $F_{-i}$ as follows: 


$$
\begin{gathered}
F_{i}=\left[F_{1}, F_{i-1}\right] \\
F_{-i}=\left\{y \in \mathfrak{g}_{-i t} \mid\left[y, F_{1}\right] \subseteq F_{-i+1}\right\} .
\end{gathered}
$$

Then $F=\bigoplus_{j} F_{j}$ is a graded subalgebra of $\mathfrak{g}$.

Proposition 1.24. Let $F=\bigoplus_{j} F_{j}$ be any graded Lie algebra satisfying (1.22), and assume that $F_{1}$ is a nontrivial $F_{0}$-module under the adjoint action. Set $A_{i}=0$ for all $i>0$. For $i \geq 0$, define $A_{-i}=\left\{y \in F_{-i} \mid\left[y, F_{1}\right] \subseteq\right.$ $\left.A_{-i+1}\right\}$. Then $A=\bigoplus_{j} A_{j}$ is a graded ideal of $F$. Moreover in the graded Lie algebra $\mathcal{B}=\bigoplus_{i} \mathcal{B}_{i}=F / A$, conditions (1.3) (1-transitivity) and (1.22) hold.

There are several important instances of this construction that will play a crucial role in the remainder of the work. For the graded Lie algebra $\mathfrak{g}=\bigoplus_{i=-q}^{r} \mathfrak{g}_{i}$, and for $1 \leq t \leq q$, the ingredients in this special case are the following:

$$
F_{-i}=\left[F_{-1}, F_{-i+1}\right] \text { and } F_{i}=\left\{y \in \mathfrak{g}_{i t} \mid\left[y, F_{-1}\right] \subseteq F_{i-1}\right\}=\mathfrak{g}_{i t} \quad(i \geq 1),
$$

$$
\begin{gathered}
A_{0}=\operatorname{Ann}_{\mathfrak{g}_{0}}\left(F_{-1}\right)=\left\{x \in \mathfrak{g}_{0} \mid\left[x, F_{-1}\right]=0\right\}, \\
A_{i}=\left\{x \in \mathfrak{g}_{i t} \mid\left[x, F_{-1}\right] \subseteq A_{i-1}\right\} \quad \text { for } i \geq 1, \\
F=F\left(V_{-t}\right)=\bigoplus_{j} F_{j} \text { and } A=A\left(V_{-t}\right)=\bigoplus_{i \geq 0} A_{i}, \\
\mathcal{B}=\mathcal{B}\left(V_{-t}\right)=F\left(V_{-t}\right) / A\left(V_{-t}\right) .
\end{gathered}
$$

Usually we take $V_{-t}$ to be an irreducible $\mathfrak{g}_{0}$-submodule of $\mathfrak{g}_{-t}$, although this is not required for the construction. We try to make choices of $t$ and submodules $V_{-t}$ that enable us to show that the homogeneous components $\mathcal{B}_{0}$ and $\mathcal{B}_{1}$ of $\mathcal{B}$ are both nonzero, for then the graded algebra $\mathcal{B}$ has at least three homogeneous components, and we can deduce much more information about the structure of the graded algebra $\mathcal{B}$. When $t>\lfloor q / 2\rfloor$, the algebra $\mathcal{B}$ has depth one. This proves very helpful as it allows us to transfer results about depth-one algebras to general graded Lie algebras.

The analogous construction starting from a $\mathfrak{g}_{0}$-submodule $V_{t}$ of $\mathfrak{g}_{t}$, for $1 \leq t \leq r$, has the following constituents:

$$
F_{0}=\mathfrak{g}_{0} \text { and } F_{1}=V_{t} \text {, a nontrivial } \mathfrak{g}_{0} \text {-submodule of } \mathfrak{g}_{t},
$$


$(1.32)$

$F_{i}=\left[F_{1}, F_{i-1}\right]$ and $\quad F_{-i}=\left\{y \in \mathfrak{g}_{-i t} \mid\left[y, F_{1}\right] \subseteq F_{-i+1}\right\}=\mathfrak{g}_{-i t} \quad(i \geq 1)$,

$$
\begin{gathered}
A_{0}=\operatorname{Ann}_{\mathfrak{g}_{0}}\left(F_{1}\right)=\left\{x \in \mathfrak{g}_{0} \mid\left[x, F_{1}\right]=0\right\} \\
A_{-i}=\left\{x \in \mathfrak{g}_{-i t} \mid\left[x, F_{1}\right] \subseteq A_{-i+1}\right\} \quad \text { for } i \geq 1 \\
F=F\left(V_{t}\right)=\bigoplus_{j} F_{j} \text { and } A=A\left(V_{t}\right)=\bigoplus_{i \geq 0} A_{-i} \\
\mathcal{B}=\mathcal{B}\left(V_{t}\right)=F\left(V_{t}\right) / A\left(V_{t}\right)
\end{gathered}
$$

REMARKS 1.37. When $t>\lfloor r / 2\rfloor$, the algebra $\mathcal{B}\left(V_{t}\right)$ constructed in (1.31)-(1.36) has height one and is 1-transitive by Proposition 1.24. By assigning $\mathcal{B}\left(V_{t}\right)$ the opposite grading where $\mathcal{B}\left(V_{t}\right)_{-i}$ is set equal to $\mathcal{B}\left(V_{t}\right)_{i}$, we obtain a depth-one, transitive algebra, which is irreducible if and only if the module $V_{t}$ is irreducible. When speaking about $\mathcal{B}\left(V_{t}\right)$ in subsequent sections, we always assume it is graded with the opposite grading and hence always regard it as a depth-one algebra.

\subsection{The local subalgebra}

There is yet another subalgebra of a graded Lie algebra $\mathfrak{g}=\bigoplus_{i=-q}^{r} \mathfrak{g}_{i}$ which plays a prominent role. This is the subalgebra $\widehat{\mathfrak{g}}$ of $\mathfrak{g}$ generated by the local part $\mathfrak{g}_{-1} \oplus \mathfrak{g}_{0} \oplus \mathfrak{g}_{1}$. Thus,

$$
\widehat{\mathfrak{g}}=\bigoplus_{i=-q^{\prime}}^{r^{\prime}} \widehat{\mathfrak{g}}_{i}
$$

where $\widehat{\mathfrak{g}}_{i}=\mathfrak{g}_{i}$ for $i=-1,0,1, \widehat{\mathfrak{g}}_{-j}=\left[\widehat{\mathfrak{g}}_{-j+1}, \widehat{\mathfrak{g}}_{-1}\right]$, and $\widehat{\mathfrak{g}}_{i}=\left[\widehat{\mathfrak{g}}_{i-1}, \widehat{\mathfrak{g}}_{1}\right]$ for all $i, j \geq 2$.

Proposition 1.39. Let $\widehat{\mathfrak{g}}$ be the Lie algebra generated by the local part $\mathfrak{g}_{-1} \oplus \mathfrak{g}_{0} \oplus \mathfrak{g}_{1}$ of the graded algebra $\mathfrak{g}$. Set $\mathcal{A}^{-}=\bigoplus_{j} \mathcal{A}_{j}^{-}$where $\mathcal{A}_{-i}^{-}=0$ for $i>0$ and $\mathcal{A}_{i}^{-}=\left\{y \in \widehat{\mathfrak{g}}_{i} \mid\left[y, \widehat{\mathfrak{g}}_{-1}\right] \subseteq \mathcal{A}_{i-1}^{-}\right\}$for all $i \geq 0$. Similarly, let $\mathcal{A}^{+}=\bigoplus_{j} \mathcal{A}_{j}^{+}$where $\mathcal{A}_{i}^{+}=0$ for $i>0$ and $\mathcal{A}_{-i}^{+}=\left\{y \in \widehat{\mathfrak{g}}_{-i} \mid\left[y, \widehat{\mathfrak{g}}_{1}\right] \subseteq \mathcal{A}_{-i+1}^{+}\right\}$ for all $i \geq 0$. Then $\widehat{\mathfrak{g}} / \mathcal{A}^{-}$is transitive and $\widehat{\mathfrak{g}} / \mathcal{A}^{+}$is 1 -transitive. Thus, $\widehat{\mathfrak{g}}$ is transitive (1.2) (respectively, 1-transitive (1.3)) if and only if $\mathcal{A}^{-}=0$ (respectively, $\mathcal{A}^{+}=0$ ).

Proof. The statements about the transitivity of $\widehat{\mathfrak{g}} / \mathcal{A}^{-}$and the 1-transitivity of $\widehat{\mathfrak{g}} / \mathcal{A}^{+}$follow directly from Propositions 1.20 and 1.24. Now if $y \in \widehat{\mathfrak{g}}_{i}$ for some $i \geq 0$, then $y \in \mathcal{A}_{i}^{-}$if and only if $\left(\operatorname{ad} \widehat{\mathfrak{g}}_{-1}\right)^{i+1}(y)=0$. If $\widehat{\mathfrak{g}}$ is transitive, then $\left(\operatorname{ad} \widehat{\mathfrak{g}}_{-1}\right)^{i+1}(y)=0$ gives $y=0$. Thus, transitivity (1.2) implies $\mathcal{A}_{i}^{-}=0$ 
for all $i \geq 0$. Conversely, when $\mathcal{A}_{i}^{-}=0$ for all $i \geq 0$, then $\widehat{\mathfrak{g}}=\widehat{\mathfrak{g}} / \mathcal{A}^{-}$is transitive. Similar arguments yield the other assertions.

\subsection{General properties of graded Lie algebras}

In this section we will derive some very general properties of graded Lie algebras $\mathfrak{g}=\bigoplus_{i=-q}^{r} \mathfrak{g}_{i}$ which satisfy

(1.40) (i) $\mathfrak{g}$ is irreducible (1.1) and transitive (1.2);

(ii) $\mathcal{M}(\mathfrak{g})=0$;

(iii) $\mathfrak{g}_{-}$is generated by $\mathfrak{g}_{-1}$; and

(iv) $\mathfrak{g}$ is finite-dimensional over a field $\mathbb{F}$ of characteristic $\neq 2,3$.

Our first objective will be to show that transitivity holds in all of $\mathfrak{g} \backslash \mathfrak{g}-q$. Hence we prove for any $x \in \mathfrak{g} \backslash \mathfrak{g}_{-q}$ that $\left[x, \mathfrak{g}_{-1}\right] \neq 0$. We may assume $x=$ $x_{-q}+\cdots+x_{j}$, where $x_{i} \in \mathfrak{g}_{i}$ for all $i$, and $x_{j} \neq 0$. It follows that $\left[x_{j}, \mathfrak{g}_{-1}\right]=0$. As $\mathfrak{g}$ is assumed to be transitive, we may suppose $-q+1 \leq j \leq-1$. Then since $\mathfrak{g}_{-}$is generated by $\mathfrak{g}_{-1}$,

$$
J:=\sum_{k, \ell \geq 0}\left(\operatorname{ad} \mathfrak{g}_{+}\right)^{k}\left(\operatorname{ad} \mathfrak{g}_{0}\right)^{\ell} x_{j}
$$

would be a graded ideal of $\mathfrak{g}$. But $j \geq-q+1$, so $J$ cannot contain the unique minimal graded ideal $\mathcal{J}$, to contradict Lemma 1.16. Thus, we have demonstrated the first part of the next result.

Lemma 1.41. Suppose $\mathfrak{g}=\bigoplus_{j=-q}^{r} \mathfrak{g}_{j}$ is a graded Lie algebra satisfying (i)-(iii) of (1.40). Then the following hold:

(a) If $x \in \mathfrak{g} \backslash \mathfrak{g}_{-q}$, then $\left[x, \mathfrak{g}_{-1}\right] \neq 0$.

(b) $\mathfrak{g}_{-q}$ is an irreducible $\mathfrak{g}_{0}$-module.

(c) If $K$ is an ideal of $\mathfrak{g}$ and $K \cap\left(\mathfrak{g}_{-1} \oplus \mathfrak{g}_{0} \oplus \mathfrak{g}_{1}\right) \neq 0$, then $K \supseteq \mathfrak{g}_{-}$.

Proof. For (b), note that similar to the argument above, if $M$ is any proper $\mathfrak{g}_{0}$-submodule of $\mathfrak{g}_{-q}$, then

$$
J:=\sum_{k, \ell \geq 0}\left(\operatorname{ad} \mathfrak{g}_{+}\right)^{k}\left(\operatorname{ad} \mathfrak{g}_{0}\right)^{\ell} M
$$

is a graded ideal of $\mathfrak{g}$, which fails to contain $\mathfrak{g}_{-q}$, hence fails to contain the unique minimal graded ideal $\mathcal{J}$. To establish (c), observe that when $K$ is an ideal such that $K \cap\left(\mathfrak{g}_{-1} \oplus \mathfrak{g}_{0} \oplus \mathfrak{g}_{1}\right) \neq 0$, then by transitivity (1.2), $K \cap \mathfrak{g}_{-1} \neq 0$. By irreducibility (1.1), $K \cap \mathfrak{g}_{-1}=\mathfrak{g}_{-1}$, and since $\mathfrak{g}_{-}$is generated by $\mathfrak{g}_{-1}, K$ must contain $\mathfrak{g}_{-}$. 
Suppose now that $V_{-t}$ is any irreducible $\mathfrak{g}_{0}$-submodule of $\mathfrak{g}_{-t}$, where $q / 2<t \leq q$. (For example, $V_{-q}=\mathfrak{g}_{-q}$ is a choice we have in mind in view of Lemma 1.41 (b).) As in (1.25)-(1.30),

$$
\begin{gathered}
F=F\left(V_{-t}\right)=\bigoplus_{i \geq-1} F_{i}, \text { where } F_{-1}=V_{-t}, \\
\quad F_{-j}=\left[F_{-1}, F_{-j+1}\right], \text { and } F_{i}=\mathfrak{g}_{i t} \text { for } i \geq 0, \\
A=A\left(V_{-t}\right)=\bigoplus_{i \geq-1} A_{i}, \text { where } A_{-1}=0 \text { and } \\
\quad A_{i}=\left\{y \in F_{i} \mid\left[y, F_{-1}\right] \subseteq A_{i-1}\right\} \text { for } i \geq 0, \\
\mathcal{B}=\mathcal{B}\left(V_{-t}\right)=\bigoplus_{i} \mathcal{B}_{i}=F / A .
\end{gathered}
$$

The algebra $\mathcal{B}$ satisfies (1.1) (irreducibility), (1.2) (transitivity), and (1.17). Note that $A_{j}=\left\{x \in \mathfrak{g}_{j t} \mid\left(\operatorname{ad} V_{-t}\right)^{j+1} x=0\right\}$, and $\mathcal{B}_{j} \neq 0 \Longleftrightarrow A_{j} \neq F_{j} \Longleftrightarrow$ $\left(\operatorname{ad} V_{-t}\right)^{j+1} \mathfrak{g}_{t j} \neq 0$. In particular,

$$
\mathcal{B}_{1} \neq 0 \Longleftrightarrow\left(\operatorname{ad} V_{-t}\right)^{2} \mathfrak{g}_{t} \neq 0
$$

Next we will set $F=F\left(\mathfrak{g}_{-q}\right), A=A\left(\mathfrak{g}_{-q}\right)$, and $\mathcal{B}=\mathcal{B}\left(\mathfrak{g}_{-q}\right)=F / A=$ $\bigoplus_{i} \mathcal{B}_{i}$, and we will argue that $\mathcal{B}_{1} \neq 0$ when $q \leq r$. But to accomplish this, we require some additional information about the action of ad $\mathfrak{g}_{-q}$ on the various spaces $\mathfrak{g}_{i}$. Clearly, ad $\mathfrak{g}_{-q}$ acts nilpotently on $\mathfrak{g}$.

LEMmA 1.45. Suppose $\mathfrak{g}=\bigoplus_{j=-q}^{r} \mathfrak{g}_{j}$ is a graded Lie algebra satisfying (i)-(iii) of (1.40). Let $\mathcal{J}=\bigoplus_{j=-q}^{s} \mathcal{J}_{j}$ be the unique minimal graded ideal of $\mathfrak{g}$ as in Lemma 1.16, and assume $\ell \geq 0$ satisfies $\ell q-q \leq s$. Then $\left(\operatorname{ad} \mathfrak{g}_{-q}\right)^{\ell_{\mathcal{J}}}=$ 0 if and only if $\left(\operatorname{ad} \mathfrak{g}_{-q}\right)^{\ell} \mathcal{J}_{j}=0$ for some $j$ such that $\ell q-q \leq j \leq s$.

Proof. What is being asserted in this rather technical lemma is that if $\left(\text { ad } \mathfrak{g}_{-q}\right)^{\ell} \mathcal{J}_{j}=0$ for some $j \geq \ell q-q$ (i.e., $j$ is large enough so that $\left(\operatorname{ad} \mathfrak{g}_{-q}\right)^{\ell} \mathcal{J}_{j}$ $=0$ is not an automatic consequence of having $-\ell q+j<-q)$, then $\left(\mathrm{ad} \mathfrak{g}_{-q}\right)^{\ell}$ annihilates J. To see this, suppose that

$$
\left(\operatorname{ad} \mathfrak{g}_{-q}\right)^{\ell} \mathcal{J}_{j}=0
$$

for some $j$ such that $\ell q-q \leq j \leq s$. Then it is necessary to show that $\left(\operatorname{ad} \mathfrak{g}_{-q}\right)^{\ell} \mathcal{J}=0$, which we will do by proving that $\left(\operatorname{ad} \mathfrak{g}_{-q}\right)^{\ell} \mathcal{J}_{k}=0$ for all $k$ with $-q \leq k \leq s$. Indeed if $-q \leq k<\ell q-q$, then $\left(\text { ad } \mathfrak{g}_{-q}\right)^{\ell} \mathfrak{J}_{k} \subseteq \mathfrak{g}_{k-q \ell}=0$. To prove the result for $\ell q-q \leq k \leq s$, we distinguish two cases: (1) $\ell q-q$ $\leq k<j$ and (2) $j<k \leq s$.

In case (1), we have by assumption and Lemma 1.16 that

$$
0=\left(\operatorname{ad} \mathfrak{g}_{-1}\right)^{j-k}\left(\operatorname{ad} \mathfrak{g}_{-q}\right)^{\ell} \mathfrak{J}_{j}=\left(\operatorname{ad} \mathfrak{g}_{-q}\right)^{\ell}\left(\operatorname{ad} \mathfrak{g}_{-1}\right)^{j-k} \mathcal{J}_{j}=\left(\operatorname{ad} \mathfrak{g}_{-q}\right)^{\ell} \mathfrak{J}_{k}
$$


In the second case,

$$
\left(\operatorname{ad} \mathfrak{g}_{-1}\right)^{k-j}\left(\operatorname{ad} \mathfrak{g}_{-q}\right)^{\ell} \mathcal{J}_{k}=\left(\operatorname{ad} \mathfrak{g}_{-q}\right)^{\ell}\left(\operatorname{ad} \mathfrak{g}_{-1}\right)^{k-j} \mathcal{J}_{k}=\left(\operatorname{ad} \mathfrak{g}_{-q}\right)^{\ell} \mathcal{J}_{j}=0
$$

must hold so that by Lemma 1.41 (a), $\left(\operatorname{ad} \mathfrak{g}_{-q}\right)^{\ell} \mathcal{J}_{k}=0$. The other direction is obvious.

In view of $(1.44)$, showing $\mathcal{B}_{1} \neq 0$ for $\mathcal{B}=\mathcal{B}\left(\mathfrak{g}_{-q}\right)$ is equivalent to showing that $\left(\operatorname{ad} \mathfrak{g}_{-q}\right)^{2} \mathfrak{g}_{q} \neq 0$. As a starting point we have

LEMMA 1.46. Under the assumptions of (1.40), $\left(\operatorname{ad} \mathfrak{g}_{-q}\right) \mathcal{J}_{j} \neq 0$ for $0 \leq j \leq s$, where $\mathcal{J}=\bigoplus_{j=-q}^{s} \mathcal{J}_{j}$ is the unique minimal graded ideal of $\mathfrak{g}$ as in Lemma 1.16. In particular, since $\mathfrak{g}$ is irreducible, $\left[\mathfrak{g}_{-q}, \mathcal{J}_{q-1}\right]=\mathfrak{g}_{-1}$ whenever $s \geq q-1$.

Proof. If $\left(\operatorname{ad~} \mathfrak{g}_{-q}\right) \mathcal{J}_{j}=0$ for some $j, 0 \leq j \leq s$, then by Lemma 1.45 (with $\ell=1$ ), we have $\left(\operatorname{ad} \mathfrak{g}_{-q}\right) \mathcal{J}=0$. Since $\mathfrak{g}_{-}$is assumed to be generated by $\mathfrak{g}_{-1}$, and since $\left[\mathfrak{g}_{-1}, \mathfrak{g}_{+}\right] \subseteq \mathcal{J}$, it follows that

$$
\sum_{i \geq 1}\left(\operatorname{ad} \mathfrak{g}_{+}\right)^{i \mathcal{J}_{-q}}
$$

is an ideal of $\mathfrak{g}$ not containing $\mathcal{J}_{-q}=\mathfrak{g}_{-q}$, and hence properly contained in $\mathcal{J}$, to contradict the minimality of $\mathcal{J}$.

REMARK 1.47. To complete the proof that $\mathcal{B}_{1} \neq 0$ for $\mathcal{B}=\mathcal{B}\left(\mathfrak{g}_{-q}\right)$ when $q \leq r$, we use the notion of a weakly closed set. Recall that a subset $\mathcal{E}$ of the endomorphism algebra $\operatorname{End}(W)$ on a vector space $W$ over $\mathbb{F}$ is weakly closed if for every ordered pair $(x, y) \in \mathcal{E}^{2}$, there is a scalar $f(x, y) \in \mathbb{F}$ such that $x y+f(x, y) y x \in \mathcal{E}$. If $\mathcal{E}$ is nil (that is, every $x \in \mathcal{E}$ is a nilpotent transformation), then the associative subalgebra of $\operatorname{End}(W)$ generated by $\mathcal{E}$ is nilpotent (see [J, p. 33]).

Lemma 1.48. Let $U$ and $V$ be $\mathfrak{g}_{0}$-submodules of $\mathfrak{g}$ such that $[U, V] \subseteq \mathfrak{g}_{0}$ and $[U,[U, V]]=0$. Let $M$ be a $\mathfrak{g}_{0}$-submodule of $\mathfrak{g}$. Then the following are true:

(i) $\operatorname{ad}_{M}[U, V]:=\left\{\left.\operatorname{ad}[u, v]\right|_{M} \mid u \in U, v \in V\right\}$ is a weakly closed subset of $\operatorname{End}(M)$.

(ii) If $\operatorname{ad}_{M}[U, V]$ consists of nilpotent transformations, then the associative subalgebra of $\operatorname{End}(M)$ generated by $\operatorname{ad}_{M}[U, V]$ acts nilpotently on $M$. If in addition $M$ is an irreducible $\mathfrak{g}_{0}$-module, then $[[U, V], M]=0$. 
Proof. Let $u_{1}$ and $u_{2}$ be any elements of $U$, and let $v_{1}$ and $v_{2}$ be any elements of $V$. Then we have

$$
\begin{aligned}
{\left[\operatorname{ad}\left[u_{1}, v_{1}\right], \operatorname{ad}\left[u_{2}, v_{2}\right]\right] } & =\operatorname{ad}\left[\left[u_{1}, v_{1}\right],\left[u_{2}, v_{2}\right]\right] \\
& =\operatorname{ad}\left[\left[\left[u_{1}, v_{1}\right], u_{2}\right], v_{2}\right]+\operatorname{ad}\left[u_{2},\left[\left[u_{1}, v_{1}\right], v_{2}\right]\right] \\
& =\operatorname{ad}\left[u_{2},\left[\left[u_{1}, v_{1}\right], v_{2}\right]\right] .
\end{aligned}
$$

Since $\left[u_{1}, v_{1}\right] \in \mathfrak{g}_{0}$ and $V$ is assumed to be a $\mathfrak{g}_{0}$-submodule of $\mathfrak{g}$, it follows that $\left[\left[u_{1}, v_{1}\right], v_{2}\right] \in V$, so ad ${ }_{M}[U, V]$ is weakly closed. Thus if ad ${ }_{M}[U, V]$ consists of nilpotent transformations, then the associative subalgebra of $\operatorname{End}(M)$ generated by ad ${ }_{M}[U, V]$ is nilpotent. But then $\operatorname{Ann}_{M}([U, V]) \neq 0$. Since $\operatorname{Ann}_{M}([U, V])$ is a $\mathfrak{g}_{0}$ submodule of $M$, it must equal $M$ whenever $M$ is irreducible, implying $[[U, V], M]=0$ in this case.

In the next sequence of results, as in Lemma 1.48, we adopt the notation $\operatorname{ad}_{V}(U)$ (respectively $\operatorname{ad}_{V} u$ ) to indicate the restriction of ad $U$ (respectively ad $u$ ) to the subspace $V$.

We now have the requisites for showing that $\mathcal{B}_{1} \neq 0$ when $q \leq r$ for $\mathcal{B}=$ $\mathcal{B}\left(\mathfrak{g}_{-q}\right)$, or equivalently, that $\left(\mathrm{ad} \mathfrak{g}_{-q}\right)^{2} \mathfrak{g}_{q} \neq 0$ when $\mathfrak{g}$ is finite-dimensional. Suppose to the contrary that $\left(\operatorname{ad} \mathfrak{g}_{-q}\right)^{2} \mathfrak{g}_{q}=0$. If $q \leq s$, Lemma 1.45 (with $\ell=2$ ) implies that $\left(\text { ad } \mathfrak{g}_{-q}\right)^{2} \mathcal{J}=0$. If instead $s<q$, then because $r-1 \leq s<q \leq r$, we must have $s=r-1=q-1$, so again we have $\left(\operatorname{ad} \mathfrak{g}_{-q}\right)^{2} \mathcal{J} \subseteq \mathfrak{g}_{-q-1} \oplus \mathfrak{g}_{-q-2} \oplus \cdots=0$. In any event, for all $y \in \mathfrak{g}_{-q}$ and $z \in \mathfrak{g}_{q}$,

$$
0=(\operatorname{ad} y)^{2}(\operatorname{ad} z)^{2} \mathfrak{g}_{-1}=2(\operatorname{ad}[y, z])^{2} \mathfrak{g}_{-1},
$$

because $\mathfrak{g}_{-1}=\mathcal{J}_{-1}$ and $\left[\mathfrak{g}_{-q}, \mathfrak{g}_{-1}\right]=0=\left(\operatorname{ad} \mathfrak{g}_{-q}\right)^{2} \mathcal{J}$. Since by Lemma 1.48 (i),

$$
Y:=\left\{\operatorname{ad}_{\mathfrak{g}_{-1}}[y, z] \mid y \in \mathfrak{g}_{-q}, z \in \mathfrak{g}_{q}\right\}
$$

is a weakly closed set, and since it consists of nilpotent transformations of $\mathfrak{g}_{-1}$, the associative subalgebra of $\operatorname{End}\left(\mathfrak{g}_{-1}\right)$ generated by $Y$ acts nilpotently on $\mathfrak{g}_{-1}$. As $\mathfrak{g}_{-1}$ is $\mathfrak{g}_{0}$-irreducible, Lemma 1.48 (ii) implies that $\left[\left[\mathfrak{g}_{-q}, \mathfrak{g}_{q}\right], \mathfrak{g}_{-1}\right]$ $=0$. By transitivity $(1.2),\left[\mathfrak{g}_{-q}, \mathfrak{g}_{q}\right]=0$. Thus, $\left[\mathfrak{g}_{-q}, \mathcal{J}_{q-1}\right] \subseteq\left[\mathfrak{g}_{-q},\left[\mathfrak{g}_{-1}, \mathfrak{g}_{q}\right]\right]=$ 0 , to contradict Lemma 1.46. Thus, we have established

Lemma 1.49. Suppose $\mathfrak{g}$ is a graded Lie algebra satisfying the conditions of (1.40). If $q \leq r$, then $\left(\operatorname{ad} \mathfrak{g}_{-q}\right)^{2} \mathfrak{g}_{q} \neq 0$. Consequently, $\mathcal{B}_{1} \neq 0$ for $\mathcal{B}=\mathcal{B}\left(\mathfrak{g}_{-q}\right)=\bigoplus_{i} \mathcal{B}_{i}$.

Our next goal is to prove when $q \geq 2$ that $\mathcal{B}_{1}^{\prime}$ is nonzero for $\mathcal{B}^{\prime}=$ $\mathcal{B}\left(V_{-q+1}\right)$, where $V_{-q+1}$ is any irreducible $\mathfrak{g}_{0}$-submodule of $\mathfrak{g}_{-q+1}$. This will require some results showing that annihilators in $\mathfrak{g}_{0}$ of pairs of spaces $\mathfrak{g}_{j}$ and $\mathfrak{g}_{j+1}$ intersect trivially. In fact, we prove a slightly more general result. For $-q \leq j \leq r-1$, let $V_{j+1}$ be any nonzero $\mathfrak{g}_{0}$-submodule of $\mathfrak{g}_{j+1}$, and set 
$Q=\operatorname{Ann}_{\mathfrak{g}_{0}}\left(\mathcal{J}_{j}\right) \cap \operatorname{Ann}_{\mathfrak{g}_{0}}\left(V_{j+1}\right)$. Under the assumption $Q \neq 0$, transitivity (1.2) and irreducibility (1.1) force $\left[Q, \mathfrak{g}_{-1}\right]=\mathfrak{g}_{-1}$. Then

$$
\left[\mathfrak{g}_{-1}, V_{j+1}\right]=\left[\left[Q, \mathfrak{g}_{-1}\right], V_{j+1}\right]=\left[Q,\left[\mathfrak{g}_{-1}, V_{j+1}\right]\right] \subseteq\left[Q, \mathcal{J}_{j}\right]=0
$$

to contradict transitivity (we have used the inclusion $\mathfrak{g}_{-1} \subset \mathcal{J}$ ). Consequently, we have

Lemma 1.50. Assume $q \geq 2$ and the hypotheses of (1.40) hold. If $V_{j+1} \neq 0$ is a $\mathfrak{g}_{0}$-submodule of $\mathfrak{g}_{j+1}$ for some $j$ such that $-q \leq j \leq r-1$, then $\operatorname{Ann}_{\mathfrak{g}_{0}}\left(\mathcal{J}_{j}\right) \cap \operatorname{Ann}_{\mathfrak{g}_{0}}\left(V_{j+1}\right)=0$.

LEMMA 1.51. Under the assumptions of (1.40), if $q \geq 2$ and $V_{-q+1} \neq 0$ is a $\mathfrak{g}_{0}$-submodule of $\mathfrak{g}_{-q+1}$, then $\operatorname{Ann}_{\mathfrak{g}_{j}}\left(\mathfrak{g}_{-q}\right) \cap \operatorname{Ann}_{\mathfrak{g}_{j}}\left(V_{-q+1}\right)=0$ for all $j$ such that $0 \leq j \leq r-1$.

Proof. Suppose $N:=\operatorname{Ann}_{\mathfrak{g}_{j}}\left(\mathfrak{g}_{-q}\right) \cap \operatorname{Ann}_{\mathfrak{g}_{j}}\left(V_{-q+1}\right)$. Then

$$
\left[\mathfrak{g}_{-q},\left[\mathfrak{g}_{-1}, N\right]\right]=\left[\mathfrak{g}_{-1},\left[\mathfrak{g}_{-q}, N\right]\right]=0
$$

In addition, we have that $\left[V_{-q+1},\left[\mathfrak{g}_{-1}, N\right]\right] \subseteq\left[\mathfrak{g}_{-q}, N\right]+\left[\mathfrak{g}_{-1},\left[V_{-q+1}\right.\right.$, $N]]=0$. Hence $\left[\mathfrak{g}_{-1}, N\right] \subseteq \operatorname{Ann}_{\mathfrak{g}_{j-1}}\left(\mathfrak{g}_{-q}\right) \cap \operatorname{Ann}_{\mathfrak{g}_{j-1}}\left(V_{-q+1}\right)$. Repeating this argument $j-1$ times, we determine that $\left(\operatorname{ad} \mathfrak{g}_{-1}\right)^{j} N \subseteq \operatorname{Ann}_{\mathfrak{g}_{0}}\left(\mathfrak{g}_{-q}\right) \cap$ $\operatorname{Ann}_{\mathfrak{g}_{0}}\left(V_{-q+1}\right)$, which is zero by Lemma 1.50. By transitivity $(1.2), N=0$ as claimed.

In order to show that $\mathcal{B}_{1}^{\prime} \neq 0$ where $\mathcal{B}^{\prime}=\mathcal{B}\left(V_{-q+1}\right)$ for some irreducible $\mathfrak{g}_{0}$-submodule $V_{-q+1}$ of $\mathfrak{g}_{-q+1}$, it will suffice to argue that $\left(\operatorname{ad} V_{-q+1}\right)^{2} \mathfrak{g}_{q-1}$ $\neq 0$ (compare (1.44)). At this stage we can prove

LEMMA 1.52. With assumptions as in (1.40), if $q \geq 2$, then $\operatorname{Ann}_{\mathfrak{g}_{q-1}}\left(V_{-q+1}\right)=0$ for any irreducible $\mathfrak{g}_{0}$-submodule $V_{-q+1}$ of $\mathfrak{g}_{-q+1}$.

Proof. Set $N:=\operatorname{Ann}_{\mathfrak{g}_{q-1}}\left(V_{-q+1}\right)$, and assume that $N \neq 0$. Then, by Lemma 1.51, $\left[\mathfrak{g}_{-q}, N\right] \neq 0$, and so by irreducibility $(1.1),\left[\mathfrak{g}_{-q}, N\right]=\mathfrak{g}_{-1}$. But then

$$
\left[\mathfrak{g}_{-1}, V_{-q+1}\right]=\left[\left[\mathfrak{g}_{-q}, N\right], V_{-q+1}\right]=\left[\mathfrak{g}_{-q},\left[N, V_{-q+1}\right]\right]=0
$$

to contradict Lemma 1.41 (a).

From Lemmas 1.41 (b) and 1.46, we know that $\mathfrak{g}_{-q}$ is an irreducible $\mathfrak{g}_{0}$-module and that $\mathfrak{g}_{-q}=\left[\mathfrak{g}_{-q}, \mathfrak{g}_{0}\right]$. An analogous statement holds with $\mathfrak{g}_{i}$ in place of $\mathfrak{g}_{0}$ under some mild assumptions.

LEMMA 1.53. Suppose $\mathfrak{g}$ is a graded Lie algebra satisfying the conditions of (1.40). If $2 \leq q \leq r$, then $\mathfrak{g}_{-q+i}=\left[\mathfrak{g}_{-q}, \mathfrak{g}_{i}\right]$ for all $i$ such that $0 \leq i \leq q-1$. 
Proof. We may assume that $i>0$. When $q \leq r$, then by Lemma 1.46 $\left[\mathfrak{g}_{-q}, \mathfrak{g}_{q-1}\right]=\mathfrak{g}_{-1}$. Since $\mathfrak{g}_{-}$is generated by $\mathfrak{g}_{-1}$, we have

$$
\begin{aligned}
\mathfrak{g}_{-q+i} & =\left(\operatorname{ad} \mathfrak{g}_{-1}\right)^{q-i-1}\left(\mathfrak{g}_{-1}\right) \\
& =\left(\operatorname{ad} \mathfrak{g}_{-1}\right)^{q-i-1}\left[\mathfrak{g}_{-q}, \mathfrak{g}_{q-1}\right] \\
& =\left[\mathfrak{g}_{-q},\left(\operatorname{ad} \mathfrak{g}_{-1}\right)^{q-i-1} \mathfrak{g}_{q-1}\right] \subseteq\left[\mathfrak{g}_{-q}, \mathfrak{g}_{i}\right]
\end{aligned}
$$

We now have the tools for showing when $3<q \leq r$ that $\mathcal{B}_{1}^{\prime}$ is nonzero for $\mathcal{B}^{\prime}=\mathcal{B}\left(V_{-q+1}\right)$ and $V_{-q+1}$ any irreducible $\mathfrak{g}_{0}$-submodule of $\mathfrak{g}_{-q+1}$.

LEMma 1.54. Assume $\mathfrak{g}$ is a graded Lie algebra satisfying the conditions of (1.40), and suppose further that $3<q \leq r$. Let $V_{-q+1}$ be an irreducible $\mathfrak{g}_{0}$ submodule of $\mathfrak{g}_{-q+1}$. Then $\mathcal{B}_{1}^{\prime} \neq 0$ for $\mathcal{B}^{\prime}=\mathcal{B}\left(V_{-q+1}\right)=\bigoplus_{i} \mathcal{B}_{i}^{\prime}$, (equivalently $\left.\left[V_{-q+1},\left[V_{-q+1}, \mathfrak{g}_{q-1}\right]\right] \neq 0\right)$.

Proof. Suppose to the contrary that $\left[V_{-q+1},\left[V_{-q+1}, \mathfrak{g}_{q-1}\right]\right]=0$. Then it follows from that and Lemma 1.48 (i) that $Z:=\left\{\operatorname{ad}_{\mathfrak{g}_{-q}}[y, z] \mid y \in V_{-q+1}, z \in\right.$ $\left.\mathfrak{g}_{q-1}\right\}$ is a weakly closed subset of $\operatorname{End}\left(\mathfrak{g}_{-q}\right)$. First we suppose that

$$
(\operatorname{ad} y)^{3}(\operatorname{ad} z)^{3} \mathfrak{g}_{-q}=0
$$

for all $y \in V_{-q+1}$ and all $z \in \mathfrak{g}_{q-1}$. Since $\left[\mathfrak{g}_{-q+1}, \mathfrak{g}_{-q}\right]=0$, our initial assumption now implies that $0=(\operatorname{ad} y)^{3}(\operatorname{ad} z)^{3} \mathfrak{g}_{-q}=6(\operatorname{ad}[y, z])^{3} \mathfrak{g}_{-q}$. Then $Z$ consists of nilpotent transformations, and the associative subalgebra of $\operatorname{End}\left(\mathfrak{g}_{-q}\right)$ generated by $Z$ acts nilpotently on $\mathfrak{g}_{-q}$. As $\mathfrak{g}_{-q}$ is $\mathfrak{g}_{0}$-irreducible, it follows from Lemma 1.48 (ii) that $\left[\mathfrak{g}_{-q},\left[V_{-q+1}, \mathfrak{g}_{q-1}\right]\right]=0$. However then,

$$
0=\left[\mathfrak{g}_{-q},\left[V_{-q+1}, \mathfrak{g}_{q-1}\right]\right]=\left[V_{-q+1},\left[\mathfrak{g}_{-q}, \mathfrak{g}_{q-1}\right]\right]=\left[V_{-q+1}, \mathfrak{g}_{-1}\right]
$$

to contradict Lemma 1.41 (a). Thus, assumption (1.55) has led to a contradiction, so we have from Lemma 1.41(b) that

$$
\mathfrak{g}_{-q}=\left(\operatorname{ad} V_{-q+1}\right)^{3}\left(\operatorname{ad} \mathfrak{g}_{q-1}\right)^{3} \mathfrak{g}_{-q} .
$$

Note that $V_{-q+1}$ commutes with $\mathfrak{g}_{-q+2}$ because $q>3$. By Lemma 1.53,

$$
\begin{aligned}
0 \neq \mathfrak{g}_{-q+1} & =\left[\mathfrak{g}_{1}, \mathfrak{g}_{-q}\right]=\left[\mathfrak{g}_{1},\left(\operatorname{ad} V_{-q+1}\right)^{3}\left(\operatorname{ad} \mathfrak{g}_{q-1}\right)^{3} \mathfrak{g}_{-q}\right] \\
& \subseteq\left(\operatorname{ad} V_{-q+1}\right)^{2} \mathfrak{g}_{q-1}
\end{aligned}
$$

contrary to our initial assumption. Thus $\left[V_{-q+1},\left[V_{-q+1}, \mathfrak{g}_{q-1}\right]\right] \neq 0$, as asserted. 


\subsection{Restricted Lie algebras}

Recall that a Lie algebra $L$ of prime characteristic is restricted (see $[\mathbf{J}$, Chap. V, Sec. 7] or [SF, Sec. 2.1]) if it has a mapping $[p]: L \rightarrow L, x \mapsto x^{[p]}$, satisfying

$$
\begin{aligned}
\operatorname{ad} x^{[p]} & =(\operatorname{ad} x)^{p} \\
(\xi x)^{[p]} & =\xi^{p} x^{[p]} \\
(x+y)^{[p]} & =x^{[p]}+y^{[p]}+\sum_{i=1}^{p-1} s_{i}(x, y),
\end{aligned}
$$

for all $x, y \in L, \xi \in \mathbb{F}$, where $s_{i}(x, y)$ is given by

$$
(\operatorname{ad}(t x+y))^{p-1}(x)=\sum_{i=1}^{p-1} t^{i-1} i s_{i}(x, y)
$$

in $\mathbb{F}[t] \otimes_{\mathbb{F}} L$ (tensor symbols are omitted in writing elements). The summands $s_{i}(x, y)$ are commutators in $x, y$.

The inspiration for the notion of the $[p]$-mapping comes from the ordinary $p$ th power in an associative algebra. Indeed, elements $X$ and $Y$ of an associative algebra satisfy

$$
(t X+Y)^{p}=t^{p} X^{p}+Y^{p}+\sum_{i=1}^{p-1} t^{i} s_{i}(X, Y)
$$

where $t$ is an indeterminate as above, and $s_{i}(X, Y)$ is a polynomial in $X, Y$ of total degree $p$. Differentiating both sides with respect to $t$ gives

$$
\sum_{j=0}^{p-1}(t X+Y)^{p-1-j} X(t X+Y)^{j}=\sum_{i=1}^{p-1} t^{i-1} i s_{i}(X, Y) .
$$

Now because the relation $\left(\begin{array}{c}p-1 \\ j\end{array}\right)=(-1)^{j}$ holds in characteristic $p$, and because $\operatorname{ad}(t X+Y)$ is the difference of the left multiplication and right multiplication operators of $t X+Y$ which commute, we have

$$
\begin{aligned}
(\operatorname{ad}(t X+Y))^{p-1}(X) & =\sum_{j=0}^{p-1}\left(\begin{array}{c}
p-1 \\
j
\end{array}\right)(-1)^{j}(t X+Y)^{p-1-j} X(t X+Y)^{j} \\
& =\sum_{j=0}^{p-1}(t X+Y)^{p-1-j} X(t X+Y)^{j} \\
& =\sum_{i=1}^{p-1} t^{i-1} i s_{i}(X, Y)
\end{aligned}
$$


which is the analogue of the above equation in the associative setting.

A representation $\varrho: L \rightarrow \mathfrak{g l}(V)$ of a restricted Lie algebra $(L,[p])$ is said to be restricted if $\varrho\left(x^{[p]}\right)=\varrho(x)^{p}$ for all $x \in L$. In particular, the adjoint representation of $L$ is restricted by (1.56). The next result is apparent for any restricted representation, but in fact it holds for any representation of a restricted Lie algebra.

Proposition 1.58. (Compare $[\mathbf{W K}]$.) Let $\varrho: L \rightarrow \mathfrak{g l}(V)$ be any representation of a restricted Lie algebra $(L,[p])$. Then

$$
(\varrho(x+y))^{p}-\varrho\left((x+y)^{[p]}\right)=(\varrho(x))^{p}-\varrho\left(x^{[p]}\right)+(\varrho(y))^{p}-\varrho\left(y^{[p]}\right)
$$

for all $x, y \in L$.

Proof. For elements $x$ and $y$ of a restricted Lie algebra $L$, we have from (1.57) with $X=\varrho(x), Y=\varrho(y)$, and $t=1$,

$$
\begin{aligned}
(\varrho(x+y))^{p} & =(\varrho(x))^{p}+(\varrho(y))^{p}+\sum_{k=1}^{p-1} s_{i}(\varrho(x), \varrho(y)) \\
& =(\varrho(x))^{p}+(\varrho(y))^{p}+\varrho\left(\sum_{k=1}^{p-1} s_{i}(x, y)\right) .
\end{aligned}
$$

But from (1.56) it follows that

$$
\varrho\left((x+y)^{[p]}\right)=\varrho\left(x^{[p]}\right)+\varrho\left(y^{[p]}\right)+\varrho\left(\sum_{k=1}^{p-1} s_{i}(x, y)\right)
$$

Subtracting (1.60) from (1.59), we obtain the desired relation.

REMARK 1.61. Assume $\mathbb{F}$ is an algebraically closed field of characteristic $p>0$. For any $x$ in a restricted Lie algebra $L$ over $\mathbb{F}$, the element $x^{p}-x^{[p]}$ is central in the universal enveloping algebra $\mathfrak{U}(L)$ of $L$. This can be readily seen from the fact that ad $x$, when applied to $\mathfrak{U}(L)$, is the difference of the left and right multiplication operators of $x$ on $\mathfrak{U}(L)$ and so

$$
\begin{aligned}
0 & =(\operatorname{ad} x)^{p}-\operatorname{ad}\left(x^{[p]}\right)=\left(L_{x}-R_{x}\right)^{p}-\operatorname{ad}\left(x^{[p]}\right) \\
& =\left(L_{x}\right)^{p}-\left(R_{x}\right)^{p}-\operatorname{ad}\left(x^{[p]}\right) \\
& =L_{x^{p}}-R_{x^{p}}-\operatorname{ad}\left(x^{[p]}\right)=\operatorname{ad}\left(x^{p}-x^{[p]}\right) .
\end{aligned}
$$

This result leads to two important consequences. The first is that if $L$ is finite-dimensional, then every irreducible $L$-module is finite-dimensional (see for example, $\left[\mathbf{S F}\right.$, Thm. 2.4]). The second is that $x^{p}-x^{[p]}$ must act as a scalar on any irreducible representation $\varrho: L \rightarrow \mathfrak{g l}(V)$ of $L$ (hence of $\mathfrak{U}(L)$ ) by Schur's Lemma. Let $\chi: L \rightarrow \mathbb{F}$ be such that

$$
\varrho(x)^{p}-\varrho\left(x^{[p]}\right)=\varrho\left(x^{p}-x^{[p]}\right)=\chi(x)^{p} \mathrm{id}
$$


for all $x \in L$. Then it follows from Proposition 1.58 that $\chi$ is linear. Often $\chi$ is referred to as the $p$-character of $\varrho$. The representation $\varrho$ is restricted precisely when $\chi$ is identically zero.

Subsequent sections of this chapter will be devoted to showing that under certain hypotheses on a graded Lie algebra $\mathfrak{g}=\bigoplus_{j=-q}^{r} \mathfrak{g}_{j}$, the adjoint representation of $\mathfrak{g}_{0}^{(1)}:=\left[\mathfrak{g}_{0}, \mathfrak{g}_{0}\right]$ on each homogeneous component $\mathfrak{g}_{-j}$ for $j \geq 1$ is restricted. Although a homogeneous component $\mathfrak{g}_{-j}$ may not be an irreducible $\mathfrak{g}_{0}^{(1)}$-module, nonetheless it will be shown to have a $p$-character. Our plan of attack will be to demonstrate that these $p$-characters are zero. This will be a consequence of the main theorem on restrictedness (Theorem 1.63), which we discuss next, and of Lemma 1.65 below.

\subsection{The main theorem on restrictedness (Theorem 1.63)}

With these preliminaries at hand, we turn our attention now to this chapter's main goal, which will be to prove

Theorem 1.63. Suppose $\mathfrak{g}=\bigoplus_{i=-q}^{r} \mathfrak{g}_{i}$ is a finite-dimensional irreducible, transitive graded Lie algebra over an algebraically closed field $\mathbb{F}$ of characteristic $p>3$ such that $\left[\mathfrak{g}_{1},\left[\mathfrak{g}_{-1}, \mathfrak{g}_{1}\right]\right] \neq 0$ and $\mathfrak{g}_{0}$ is a direct sum of ideals, each of which is one of the following:

(a) abelian,

(b) a classical simple Lie algebra (including, possibly, $\mathfrak{p s l}_{n}$ with $p \mid n$ ),

(c) a Lie algebra isomorphic to $\mathfrak{s l}_{n}, \mathfrak{g l}_{n}$, or $\mathfrak{p g l}_{n}$ with $p \mid n$.

Then the representation of $\mathfrak{g}_{0}^{(1)}:=\left[\mathfrak{g}_{0}, \mathfrak{g}_{0}\right]$ on $\mathfrak{g}_{-1}$ is restricted.

\subsection{Remarks on restrictedness}

In Kac's version of the Recognition Theorem in [K2], the representation of $\mathfrak{g}_{0}$ on $\mathfrak{g}_{-1}$ was assumed to be restricted. This hypothesis was shown to be unnecessary in the depth-one case in $[\mathbf{G 2}]$ (see also $[\mathbf{G 1}]$ and $[\mathbf{K u 1}]$ ), and in $[\mathbf{B G}]$, which treated the arbitrary depth case for $p>5$. Below we provide a new proof for $p>3$ based on the ideas from $[\mathbf{B G}]$ and $[\mathbf{G 2}]$. Our proof will complete the original argument in $[\mathbf{B G}]$. As shown in $[\mathbf{B K K}]$, the assumption on $p$ in the statement of Theorem 1.63 cannot be relaxed even in the depth-one case. Here is a brief outline of how the argument will proceed.

As $\mathfrak{g}_{0}$ is classical reductive, the sum $\mathfrak{g}_{0}^{\odot}$ of the nonabelian ideals is a restricted Lie algebra under a certain natural $[p]$-mapping. For every $x \in$ $\mathfrak{g}_{0}^{\odot},(\operatorname{ad} x)^{p}-\operatorname{ad} x^{[p]}$ must act as a scalar, say $\chi(x)^{p}$, on the irreducible $\mathfrak{g}_{0^{-}}$ module $\mathfrak{g}_{-1}$ (compare Remark 1.61). The representation will be restricted 
if the $p$-character $\chi(x)=0$ for all $x$. First we prove that $\chi(x)=0$ for all $x \in\left[\mathfrak{g}_{0}^{\odot}, \mathfrak{g}_{0}^{\odot}\right]=\left[\mathfrak{g}_{0}, \mathfrak{g}_{0}\right]=\mathfrak{g}_{0}^{(1)}$ in the special case that the depth $q$ of the graded Lie algebra equals one. Next we consider the depth-one Lie algebra $\bigoplus_{j \geq-1} \mathfrak{g}_{j q}$ when $q \leq r$ or the algebra $\bigoplus_{j \leq 1} \mathfrak{g}_{j r}$ when $q>r$. We also need to work with the depth-one algebra $\bigoplus_{j>-1} \mathfrak{g}_{j(q-1)}$ for $q \leq r$ or $\bigoplus_{j \leq 1} \mathfrak{g}_{j(r-1)}$ for $q>r$. Applying the depth-one result to the quotients $\mathcal{B}\left(\overline{\mathfrak{g}}_{-q}\right)$ and $\mathcal{B}\left(\mathfrak{g}_{-q+1}\right)$ of the subalgebras $\bigoplus_{j \geq-1} \mathfrak{g}_{j q}$ and $\bigoplus_{j \geq-1} \mathfrak{g}_{j(q-1)}$, which come from Proposition 1.20, (or to their counterparts when $q>r$ ), and using Lemma 1.41 (a), we conclude $q \chi(x)=0$ and $(q-1) \chi(x)=0$ for all $x$. This forces $\chi(x)=0$ for all $x \in \mathfrak{g}_{0}^{(1)}$, so that the representation is restricted as asserted.

\subsection{The action of $\mathfrak{g}_{0}$ on $\mathfrak{g}_{-j}$}

Henceforth in this chapter, we assume that $\mathfrak{g}$ is a graded Lie algebra satisfying the hypotheses of Theorem 1.63. Since by assumption, $\mathfrak{g}_{-1}$ is an irreducible $\mathfrak{g}_{0}$-module, any central element of $\mathfrak{g}_{0}$ must act as a scalar on $\mathfrak{g}_{-1}$ by Schur's Lemma. By transitivity (1.2), the center can be at most one-dimensional. Consequently, there is at most one summand of type (a), and when such a summand exists, it is the one-dimensional center of $\mathfrak{g}_{0}$. In this case, no summands isomorphic to $\mathfrak{s l}_{n}$ or $\mathfrak{g l}_{n}$ with $p \mid n$ occur. Similarly there is at most one ideal isomorphic to $\mathfrak{s l}_{n}$ or $\mathfrak{g l}_{n}$ with $p \mid n$, and if one occurs, there are no summands of the abelian type (a).

Let $\mathfrak{t}$ be the sum of the maximal toral subalgebras of the summands of $\mathfrak{g}_{0}$. Thus, $\mathfrak{t}$ contains the abelian summand if one occurs, and $\mathfrak{t}$ is a maximal toral subalgebra, hence a Cartan subalgebra, of $\mathfrak{g}_{0}$. The algebra $\mathfrak{g}_{0}$ has a root space decomposition, $\mathfrak{g}_{0}=\mathfrak{t} \oplus \bigoplus_{\alpha \in \mathfrak{t}^{*} \backslash\{0\}} \mathfrak{g}_{0}^{\alpha}$ relative to $\mathfrak{t}$, where

$$
\mathfrak{g}_{0}^{\alpha}=\left\{x \in \mathfrak{g}_{0} \mid[t, x]=\alpha(t) x \text { for all } t \in \mathfrak{t}\right\} .
$$

By [S, Lem. II.3.2], the root spaces of the summands, hence of $\mathfrak{g}_{0}$, are one-dimensional. This result will be important for us; it holds under our assumption on $p$ but may fail in characteristic 2 or 3 (this can be seen, for example, by examining the roots of the Lie algebra $\mathfrak{p s t}_{3}$ in characteristic 3 ).

It follows from [S, Lem. II.3.2] that the set of roots of $\mathfrak{g}_{0}$ relative to $\mathfrak{t}$ is obtained by mod $p$ reduction from a genuine (reduced) root system $\Phi$ in the sense of [H3, Chap. III] or [Bou1, Chap. IV-VI] (see Chapter 2 below for more detail). Let $\Delta$ be a basis of simple roots in $\Phi$. We say that a root $\alpha$ of $\mathfrak{g}_{0}$ relative to $\mathfrak{t}$ is positive (which we write $\alpha>0$ ) if $\alpha$ corresponds to an element from the positive subsystem of $\Phi$ relative to $\Delta$. A root is negative $(\alpha<0)$ if $-\alpha>0$. We suppose that the vector $e_{\alpha}$ spans the root space $\mathfrak{g}_{0}^{\alpha}$. For $\alpha>0$, we assume that the vectors $e_{\alpha}, e_{-\alpha}$, and $h_{\alpha}:=\left[e_{\alpha}, e_{-\alpha}\right]$ determine a canonical basis for a copy of $\mathfrak{s l}_{2}$. Thus,

$$
\left[h_{\alpha}, e_{\alpha}\right]=2 e_{\alpha}, \quad\left[h_{\alpha}, e_{-\alpha}\right]=-2 e_{-\alpha} .
$$


In classical algebras, there is a 'standard' $p$ th power map satisfying $e_{\alpha}^{[p]}=$ 0 for all roots $\alpha$, and $h_{\alpha}^{[p]}=h_{\alpha}$ for all $\alpha>0$. (See Chapter 2 below.) Thus, when $\mathfrak{g}$ satisfies the hypotheses of Theorem 1.63, the standard $p$ th power map on the various classical summands of $\mathfrak{g}_{0}$ can be extended to the sum $\mathfrak{g}_{0}^{\odot}$ of the nonabelian ideals giving it the structure of a restricted Lie algebra, which we assume is fixed from now on.

As in Remark 1.61, there exists a linear map $\chi: \mathfrak{g}_{0}^{\odot} \rightarrow \mathbb{F}$ such that for all $x \in \mathfrak{g}_{0}^{\odot}$,

$$
(\operatorname{ad} x)^{p}-\operatorname{ad} x^{[p]}=\chi(x)^{p} \text { id }
$$

on $\mathfrak{g}_{-1}$. Now when $\mathfrak{g}_{-j}=\left[\mathfrak{g}_{-j+1}, \mathfrak{g}_{-1}\right]$ for all $j=1, \ldots, q$, we may assume by induction that $(\operatorname{ad} x)^{p}-\operatorname{ad} x^{[p]}=(j-1) \chi(x)$ id on $\mathfrak{g}_{-j+1}$. Then since $(\operatorname{ad} x)^{p}$ and $\operatorname{ad} x^{[p]}$ are derivations, it follows from applying $(\operatorname{ad} x)^{p}-\operatorname{ad} x^{[p]}$ to $\mathfrak{g}_{-j}=\left[\mathfrak{g}_{-j+1}, \mathfrak{g}_{-1}\right]$ that $(\operatorname{ad} x)^{p}-\operatorname{ad} x^{[p]}=j \chi(x)^{p}$ id on $\mathfrak{g}_{-j}$. To summarize we have:

Lemma 1.65. (Compare [BG, Lem. 1].) Assume $\mathfrak{g}$ is a graded Lie algebra over an algebraically closed field $\mathbb{F}$ of characteristic $p>3$ satisfying the conditions of Theorem 1.63. Let $\mathfrak{g}_{0}^{\odot}$ be the sum of the nonabelian ideals of $\mathfrak{g}_{0}$.

(i) Then there is a linear functional $\chi$ on $\mathfrak{g}_{0}^{\odot}$ such that $(\operatorname{ad} x)^{p}-$ ad $x^{[p]}=\chi(x)^{p}$ id on $\mathfrak{g}_{-1}$ for all $x \in \mathfrak{g}_{0}^{\odot}$.

(ii) If $\mathfrak{g}_{-j}=\left[\mathfrak{g}_{-j+1}, \mathfrak{g}_{-1}\right]$ for $j=1, \ldots, q$, then $(\operatorname{ad} x)^{p}-\operatorname{ad} x^{[p]}=$ $j \chi(x)^{p}$ id on $\mathfrak{g}_{-j}$ for all $x \in \mathfrak{g}_{0}^{\odot}$.

When $\mathfrak{g}$ is a graded Lie algebra satisfying the conditions of Theorem 1.63 , the maximal toral subalgebra $\mathfrak{t}$ of $\mathfrak{g}_{0}$ is abelian, and so any finitedimensional $\mathfrak{g}_{0}$-module $M$ decomposes into weight spaces (common generalized eigenspaces) relative to $\mathfrak{t}$. Thus, if $\varphi: \mathfrak{g}_{0} \rightarrow \mathfrak{g l}(M)$ is the corresponding representation and $d=\operatorname{dim} M$, then $M=\bigoplus_{\lambda \in \mathfrak{t}^{*}} M^{\lambda}$ where

$$
M^{\lambda}=\left\{v \in M \mid(\varphi(t)-\lambda(t) \text { id })^{d} v=0 \text { for all } t \in \mathfrak{t}\right\} .
$$

The functional $\lambda \in \mathfrak{t}^{*}$ is a weight of $\mathfrak{t}$ on $M$ if $M^{\lambda} \neq 0$. If $v \in M^{\lambda}$ and $(\varphi(t)-\lambda(t) \mathrm{id})^{d} v=0$, then $(\varphi(t)-(\lambda+\alpha)(t) \mathrm{id})^{d} x . v=0$ for all root vectors $x \in \mathfrak{g}_{0}^{\alpha}$ so that

$$
\mathfrak{g}_{0}^{\alpha} \cdot M^{\lambda} \subseteq M^{\lambda+\alpha} .
$$

Moreover, the sum $M^{\prime}=\bigoplus_{\lambda \in \mathfrak{h}^{*}}\left(M^{\lambda}\right)^{\prime}$ of the genuine eigenspaces $\left(M^{\lambda}\right)^{\prime}=$ $\{v \in M \mid(\varphi(t)-\lambda(t)$ id $) v=0$ for all $t \in \mathfrak{t}\}$ is a nonzero $\mathfrak{g}_{0}$-submodule of $M$. As a consequence, we have the following well-known result: 
Lemma 1.67. On any (finite-dimensional) irreducible $\mathfrak{g}_{0}$-module, the maximal toral subalgebra $\mathfrak{t}$ acts semisimply.

\subsection{The depth-one case of Theorem 1.63}

We begin tackling Theorem 1.63 by first proving it when the depth $q=1$. Initially we show under the hypotheses of that theorem and the condition $q=$ 1 , that the $\mathfrak{g}_{0}$-module $\mathfrak{g}_{-1}$ possesses a common eigenvector for the adjoint action of the solvable subalgebra $\mathfrak{b}^{+}=\mathfrak{t} \oplus \bigoplus_{\alpha>0} \mathfrak{g}_{0}^{\alpha}$ of $\mathfrak{g}_{0}$ (and by symmetry, a common eigenvector for the adjoint action of $\left.\mathfrak{b}^{-}=\mathfrak{t} \oplus \bigoplus_{\alpha>0} \mathfrak{g}_{0}^{-\alpha}\right)$. In the process of establishing this, we obtain valuable information about how root vectors of $\mathfrak{g}_{0}$ act on $\mathfrak{g}_{-1}$.

From Lemma 1.67, we know that the maximal toral subalgebra $\mathfrak{t}$ acts semisimply on $\mathfrak{g}_{-1}$, say $\mathfrak{g}_{-1}=\bigoplus_{\lambda \in \mathfrak{t}^{*}} \mathfrak{g}_{-1}^{\lambda}$ is its eigenspace (weight space) decomposition. We want to prove that for some $\lambda$, there exists a nonzero $v \in \mathfrak{g}_{-1}^{\lambda}$ such that $[x, v]=0$ for all $x \in \mathfrak{g}_{0}^{\alpha}, \alpha>0$. Such a vector $v$ will generate $\mathfrak{g}_{-1}$ by irreducibility (1.1) and will be a common eigenvector for ad $\mathfrak{b}^{+}$. In particular, we show

THEOREM 1.68. Under the assumptions of Theorem 1.63 and the hypothesis that the depth $q=1$, the $\mathfrak{g}_{0}$-module $\mathfrak{g}_{-1}$ contains a common eigenvector for $\mathfrak{b}^{+}=\mathfrak{t} \oplus \bigoplus_{\alpha>0} \mathfrak{g}_{0}^{\alpha}$ (and by symmetry, for $\mathfrak{b}^{-}=\mathfrak{t} \oplus \bigoplus_{\alpha>0} \mathfrak{g}_{0}^{-\alpha}$ ).

Proof. Recall from Lemma 1.65 (i) that there is a linear functional $\chi$ on the sum $\mathfrak{g}_{0}^{\odot}$ of the nonabelian ideals such that (see (1.64)) $(\operatorname{ad} x)^{p}-\operatorname{ad} x^{[p]}=$ $\chi(x)^{p}$ id holds on $\mathfrak{g}_{-1}$ for all $x \in \mathfrak{g}_{0}^{\odot}$. If $x \in \mathfrak{g}_{0}^{\alpha}$ is a root vector of $\mathfrak{g}_{0}$, then $x^{[p]}=0$, and $(\operatorname{ad} x)^{p}=\chi(x)^{p}$ id on $\mathfrak{g}_{-1}$. If $\chi\left(e_{\alpha}\right)=0$ for all $\alpha>0$, then the subspace $\mathfrak{n}^{+}:=\bigoplus_{\alpha>0} \mathfrak{g}_{0}^{\alpha}$ is a Lie algebra acting as nilpotent transformations on $\mathfrak{g}_{-1}$, so it possesses a common eigenvector (compare Remark 1.47). As the space of those eigenvectors is invariant under ad $\mathfrak{t}$, there will be a common eigenvector for ad $\mathfrak{b}^{+}$in $\mathfrak{g}_{-1}$. We may assume then that $\chi\left(e_{\alpha}\right) \neq 0$ for some $\alpha>0$.

Let $M$ be any irreducible $\mathfrak{g}_{0}$-submodule of $\mathfrak{g}_{1}$. Then the product $P:=$ $\left[\mathfrak{g}_{-1}, M\right]$ is a nonzero ideal of $\mathfrak{g}_{0}$ by transitivity (1.2). Recall that all root spaces of $\mathfrak{g}_{0}$ relative to $\mathfrak{t}$ are one-dimensional. Hence if $P \nsubseteq \mathfrak{t}$, then $P$ contains a root vector, $e_{\gamma}$ say. In this case $e_{-\gamma} \in\left[\mathfrak{g}_{0}^{-\gamma},\left[\mathfrak{g}_{0}^{-\gamma}, e_{\gamma}\right]\right]$ is in $P$ as well. So it can be assumed further that $\gamma>0$. As the Lie algebra $\mathfrak{n}^{+}$is nilpotent, the ad $\mathfrak{n}^{+}$-module generated by $e_{\gamma}$ contains a root vector $e_{\delta}$ (with $\delta>0)$ such that $\left[\mathfrak{n}^{+}, e_{\delta}\right]=0$. If $P \subseteq \mathfrak{t}$, then each root of $\mathfrak{g}_{0}$ relative to $\mathfrak{t}$ vanishes on $P$. Since $\mathfrak{t}$ is abelian, this shows that $P$ is contained in the center $\mathfrak{Z}\left(\mathfrak{g}_{0}\right)$ of $\mathfrak{g}_{0}$. Since $\operatorname{dim} \mathfrak{Z}\left(\mathfrak{g}_{0}\right) \leq 1$, it must be that $P=\mathfrak{Z}\left(\mathfrak{g}_{0}\right)$ in this case.

If $P \nsubseteq \mathfrak{t}$, we set $e=e_{\delta}$. If $P \subseteq \mathfrak{t}$, we take for $e$ any nonzero element in $\mathfrak{Z}\left(\mathfrak{g}_{0}\right)=P$. In both cases, we let $\beta$ denote the weight of $e$ (so that 
$\beta \in\{0, \delta\})$. It follows from Lemma 1.67 that $e \in \sum_{\lambda \in \mathfrak{t}^{*}}\left[\mathfrak{g}_{-1}^{\lambda}, M^{\beta-\lambda}\right]$. For some $\lambda$, we must have $\left[\mathfrak{g}_{-1}^{\lambda}, M^{\beta-\lambda}\right]=\mathbb{F} e$ if $\beta$ is a root, because the root space is one-dimensional. Then $e=[u, v]$ for some $u \in \mathfrak{g}_{-1}^{\lambda}, v \in M^{\beta-\lambda}$. If $\beta=0$, we may assume $e$ has that form. By transitivity, $\left[\mathfrak{g}_{-1}, e\right] \neq 0$, and since $\mathfrak{g}_{-1}$ is spanned by weight vectors, there exists $w \in \mathfrak{g}_{-1}^{\nu}$ for some $\nu$ such that $[w, e] \neq 0$.

Since the depth $q=1$, we have $\left[\left(\operatorname{ad} e_{\alpha}\right)^{k}(w), u\right]=0$ for all $k \geq 0$. For $k=0,1, \ldots, p-1$ we set $x_{k}=\left[\left(\operatorname{ad} e_{\alpha}\right)^{k}(w), v\right]$ and observe that $x_{k} \in \mathfrak{g}_{0}^{\eta+k \alpha}$ where $\eta=\beta-\lambda+\nu$. Since $e$ commutes with $e_{\alpha}$ and $\left(\operatorname{ad} e_{\alpha}\right)^{p}([w, e])=$ $\chi\left(e_{\alpha}\right)^{p}[w, e]$, a nonzero multiple of $[w, e]$, it must be that

$$
\left[u, x_{k}\right]=\left[\left(\operatorname{ad} e_{\alpha}\right)^{k}(w),[u, v]\right]=\left(\operatorname{ad} e_{\alpha}\right)^{k}([w, e]) \neq 0
$$

for all $k \geq 0$. But then $\mathfrak{g}_{0}^{\eta+k \alpha} \neq 0$ for $k=0,1, \ldots, p-1$. If $\eta$ is not a multiple of $\alpha$, this is impossible, since root strings in classical Lie algebras have length at most 4 . If $\eta$ is a multiple of $\alpha$, then all multiples $k \alpha$ for $k=1, \ldots, p-1$ are roots, which again cannot happen for $p>3$ (both statements follow from the Mills-Seligman axioms which characterize direct sums of classical simple Lie algebras; see Theorem 2.5 of Chapter 2 for more details). We have reached a contradiction, so we are forced to draw the following two conclusions: Under the hypotheses of Theorem 1.63 and the assumption $q=1$,

$$
\chi(x)=0 \text { for all } x \in \mathfrak{g}_{0}^{\alpha} \text { and all } \alpha>0 ;
$$

the $\mathfrak{g}_{0}$-module $\mathfrak{g}_{-1}$ has a common eigenvector for ad $\mathfrak{b}^{+}$

By symmetrical arguments,

$$
\chi(x)=0 \text { for all } x \in \mathfrak{g}_{0}^{-\alpha} \text { and all } \alpha>0 ;
$$

the $\mathfrak{g}_{0}$-module $\mathfrak{g}_{-1}$ has a common eigenvector for ad $\mathfrak{b}^{-}$.

\subsection{Proof of Theorem 1.63 in the depth-one case}

Recall, as in the proof of Theorem 1.68, that when $\mathfrak{g}$ is a graded Lie algebra over an algebraically closed field $\mathbb{F}$ of characteristic $p>3$ satisfying the hypotheses of Theorem 1.63, there is a linear functional $\chi$ on the sum $\mathfrak{g}_{0}^{\odot}$ of the nonabelian ideals such that $(\operatorname{ad} x)^{p}-\operatorname{ad} x^{[p]}=\chi(x)^{p}$ id holds on $\mathfrak{g}_{-1}$ for all $x \in \mathfrak{g}_{0}^{\odot}$. From our proof of Theorem 1.68 we know that $\chi(x)=0$ for all root vectors $x$ of $\mathfrak{g}_{0}$ when $q=1$.

Suppose that $e=e_{\alpha} \in \mathfrak{g}_{0}^{\alpha}$ and $f=e_{-\alpha} \in \mathfrak{g}_{0}^{-\alpha}$ are such that they along with $h=[e, f] \in \mathfrak{t}$ determine a canonical basis of $\mathfrak{s l}_{2}$ as before. Then since $\exp (\operatorname{ad} e)$ is an automorphism of $\mathfrak{g}_{0}$ (see Chapter 2 to follow for the details), the element $f^{\prime}=\exp (\operatorname{ad} e)(f)=f+h-e$ is a root vector relative to the maximal toral subalgebra $\mathfrak{t}^{\prime}=\exp (\operatorname{ad} e)(\mathfrak{t})$. As above, $\chi\left(f^{\prime}\right)=0$. Then from Proposition 1.58 we deduce that on $\mathfrak{g}_{-1}$, 


$$
\begin{aligned}
0 & =\left(\operatorname{ad} f^{\prime}\right)^{p}-\operatorname{ad}\left(f^{\prime}\right)^{[p]} \\
& =(\operatorname{ad}(f+h-e))^{p}-\operatorname{ad}(f+h-e)^{[p]} \\
& =(\operatorname{ad} f)^{p}-\operatorname{ad} f^{[p]}+(\operatorname{ad} h)^{p}-\operatorname{ad} h^{[p]}-(\operatorname{ad} e)^{p}+\operatorname{ad} e^{[p]} \\
& =(\operatorname{ad} h)^{p}-\operatorname{ad} h^{[p]} .
\end{aligned}
$$

Thus, $\chi(h)=0$. Since the elements $e=e_{\alpha}, f=e_{-\alpha}, h=h_{\alpha}$, as $\alpha$ ranges over all the roots of $\mathfrak{g}_{0}$, span $\left[\mathfrak{g}_{0}^{\odot}, \mathfrak{g}_{0}^{\odot}\right]=\mathfrak{g}_{0}^{(1)}$, we may use the semilinearity of the $[p]$-mapping in $(1.56)$ to see that the representation of $\mathfrak{g}_{0}^{(1)}$ on $\mathfrak{g}_{-1}$ is restricted. Consequently, we have proven

Lemma 1.69. If $\mathfrak{g}=\bigoplus_{i=-1}^{r} \mathfrak{g}_{i}$ is a depth-one graded Lie algebra over an algebraically closed field of characteristic $p>3$ satisfying the assumptions of Theorem 1.63, then the representation of $\mathfrak{g}_{0}^{(1)}$ on $\mathfrak{g}_{-1}$ is restricted.

REMARK 1.70. If $M$ is an irreducible $\mathfrak{g}_{0}$-module, which is restricted as a $\mathfrak{g}_{0}^{(1)}$-module, then $M$ is already irreducible over $\mathfrak{g}_{0}^{(1)}$. Indeed, let $\mathfrak{U}_{[p]}\left(\mathfrak{n}^{-}\right)$ denote the restricted enveloping algebra of $\mathfrak{n}^{-}:=\bigoplus_{\alpha>0} \mathfrak{g}_{0}^{-\alpha}$, and let $J$ be the augmentation ideal of $\mathfrak{U}_{[p]}\left(\mathfrak{n}^{-}\right)$. It is well-known (and easy to see) that $J$ is the Jacobson radical of $\mathfrak{U}_{[p]}\left(\mathfrak{n}^{-}\right)$. Clearly, the subspace $J M$ is $\mathfrak{t}$-stable. By Nakayama's lemma, J.M $\neq M$. Let $v_{0}$ be a common eigenvector for $\mathfrak{b}^{+}=\mathfrak{t} \oplus \mathfrak{n}^{+}$in $M$, and let $\lambda \in \mathfrak{t}^{*}$ be the weight of $v_{0}$. Since $M=\mathfrak{U}^{[p]}\left(\mathfrak{n}^{-}\right) v_{0}$ by the irreducibility of $M$, we have $v_{0} \notin J M$. It follows that $J M$ has codimension one in $M$. Let $M_{0}=\left\{v \in M \mid \mathfrak{n}^{+} v=0\right\}$. The subspace $M_{0}$ is $\mathfrak{t}$-stable, hence decomposes into weight spaces for $\mathfrak{t}$. If $\mu \neq \lambda$, then $M_{0}^{\mu} \subseteq J M$, and hence $\mathfrak{U}_{[p]}\left(\mathfrak{n}^{-}\right) M_{0}^{\mu}$ is a $\mathfrak{g}_{0}$-submodule of $M$ contained in $J M$. Therefore, $M_{0}=M_{0}^{\lambda}$. If $\operatorname{dim} M_{0}^{\lambda}>1$, then $\mathfrak{U}_{[p]}\left(\mathfrak{n}^{-}\right)\left(M_{0}^{\lambda} \cap J M\right)$ is a nonzero $\mathfrak{g}_{0}$-submodule of $M$ contained in $J M$. Since this contradicts the irreducibility of $M$, we derive that $M_{0}=\mathbb{F} v_{0}$. As $\mathfrak{n}^{+} \subset \mathfrak{g}_{0}^{(1)}$, every $\mathfrak{g}_{0}^{(1)}$ submodule $N$ of $M$ must contain $v_{0}$. But then $N \supseteq \mathfrak{U}_{[p]}\left(\mathfrak{n}^{-}\right) v_{0}=M$, to show $M$ is irreducible over $\mathfrak{g}_{0}^{(1)}$.

\subsection{Quotients of $\mathfrak{g}_{0}$}

In demonstrating Theorem 1.63 for arbitrary depths, we will require some knowledge about quotients of $\mathfrak{g}_{0}$.

Lemma 1.71. Suppose $\mathfrak{g}=\bigoplus_{i=-q}^{r} \mathfrak{g}_{i}$ is a graded Lie algebra satisfying the hypotheses of Theorem 1.63, and let $A_{0}$ be a nonzero ideal of $\mathfrak{g}_{0}$. Then the following are true:

(i) If $\operatorname{dim} \mathfrak{Z}\left(\mathfrak{g}_{0} / A_{0}\right) \leq 1$, then the Lie algebra $\mathfrak{g}_{0} / A_{0}$ is isomorphic to a direct sum of ideals of type (a), (b), (c); 
(ii) Any solvable ideal of $A_{0}$ is contained in $\mathfrak{Z}\left(\mathfrak{g}_{0}\right)$.

Proof. (i) We know that $\mathfrak{g}_{0}=\bigoplus_{i=1}^{\ell} \mathfrak{g}_{0}^{[i]}$, where each $\mathfrak{g}_{0}^{[i]}$ is of type (a), (b), or (c). Let $\pi_{i}$ denote the canonical projection from $\mathfrak{g}_{0}$ onto $\mathfrak{g}_{0}^{[i]}$ for $1 \leq i \leq \ell$. We may assume after possibly renumbering that for some $k \geq 0$ we have $\pi_{i}\left(A_{0}\right) \nsubseteq \mathfrak{Z}\left(\mathfrak{g}_{0}^{[i]}\right)$ if $i \leq k$ and $\pi_{i}\left(A_{0}\right) \subseteq \mathfrak{Z}\left(\mathfrak{g}_{0}^{[i]}\right)$ if $k<i \leq \ell$. Since $\mathfrak{Z}\left(\mathfrak{g}_{0}^{[i]}\right) \subseteq \mathfrak{Z}\left(\mathfrak{g}_{0}\right)$, and any noncentral ideal of $\mathfrak{g}_{0}^{[i]}$ contains $\left(\mathfrak{g}_{0}^{[i]}\right)^{(1)}$, it must be that

(1.72) $\left(\mathfrak{g}_{0}^{[1]}\right)^{(1)} \oplus \cdots \oplus\left(\mathfrak{g}_{0}^{[k]}\right)^{(1)} \subseteq A_{0} \subseteq \mathfrak{g}_{0}^{[1]} \oplus \cdots \oplus \mathfrak{g}_{0}^{[k]} \oplus \mathfrak{Z}\left(\mathfrak{g}_{0}^{[k+1]}\right) \oplus \cdots \oplus \mathfrak{Z}\left(\mathfrak{g}_{0}^{[\ell]}\right)$.

Set $K:=\left(\mathfrak{g}_{0}^{[1]}\right)^{(1)} \oplus \cdots \oplus\left(\mathfrak{g}_{0}^{[k]}\right)^{(1)}$. For each $i$, let $\eta_{i}: \mathfrak{g}_{0}^{[i]} \rightarrow \mathfrak{g}_{0}^{[i]} /\left(\mathfrak{g}_{0}^{[i]}\right)^{(1)}$ be the canonical map, and set $\psi=\sum_{i=1}^{k} \eta_{i} \circ \pi_{i}+\sum_{t=k+1}^{\ell} \pi_{t}$. Then

$$
\psi: \mathfrak{g}_{0} \rightarrow Q:=\left(\bigoplus_{i=1}^{k} \mathfrak{g}_{0}^{[i]} /\left(\mathfrak{g}_{0}^{[i]}\right)^{(1)}\right) \oplus\left(\bigoplus_{t=k+1}^{\ell} \mathfrak{g}_{0}^{[t]}\right),
$$

and the kernel of $\psi$ is $K$, so there is an induced isomorphism $\bar{\psi}: \mathfrak{g}_{0} / K \rightarrow Q$. Now if $S:=\bigoplus_{i=1}^{k} \mathfrak{g}_{0}^{[i]} /\left(\mathfrak{g}_{0}^{[i]}\right)^{(1)}$, then

$$
R:=\bar{\psi}\left(A_{0} / K\right) \subseteq S \oplus \mathfrak{Z}\left(\mathfrak{g}_{0}^{[k+1]}\right) \oplus \cdots \oplus \mathfrak{Z}\left(\mathfrak{g}_{0}^{[\ell]}\right)=\mathfrak{Z}(Q) .
$$

Thus,

$$
(Q / R) /(\mathfrak{Z}(Q) / R) \cong Q / \mathfrak{Z}(Q) \cong \bigoplus_{t=k+1}^{\ell} \mathfrak{g}_{0}^{[t]} / \mathfrak{Z}\left(\mathfrak{g}_{0}^{[t]}\right),
$$

which is semisimple and is the direct sum of ideals of type (b) or (c) (only $\mathfrak{p g l}_{n}$ with $p \mid n$ is possible in case (c)). As $\mathfrak{Z}(Q) / R \subseteq \mathfrak{Z}(Q / R)$, we must have equality, $\mathfrak{Z}(Q) / R=\mathfrak{Z}(Q / R)$.

Now $\mathfrak{g}_{0} / A_{0} \cong\left(\mathfrak{g}_{0} / K\right) /\left(A_{0} / K\right) \cong Q / R$, so if $\operatorname{dim} \mathfrak{Z}\left(\mathfrak{g}_{0} / A_{0}\right) \leq 1$, then $\operatorname{dim} \mathfrak{Z}(Q) / R \leq 1$. In particular, if $R \subseteq S$, then $\mathfrak{g}_{0} / A_{0} \cong Q / R \cong S / R \oplus$ $\bigoplus_{t=k+1}^{\ell} \mathfrak{g}_{0}^{[t]}$, which is the sum of ideals of type (a), (b), or (c). If $R \nsubseteq S$, then we can write $R=(R \cap S) \oplus \mathbb{F}(s+z)$ where $s \in S, z \in \mathfrak{Z}\left(\mathfrak{g}_{0}^{[j]}\right)=$ $\mathfrak{Z}\left(\mathfrak{g}_{0}\right)$ (see the comments in Section 1.10) for some $j$, and $z \neq 0$. Moreover, $Q=(R \cap S) \oplus \mathbb{F}(s+z) \oplus \mathbb{F} s^{\prime} \oplus \bigoplus_{t=k+1}^{\ell} \mathfrak{g}_{0}^{[t]}$ for some nonzero $s^{\prime} \in S$. Thus, $\mathfrak{g}_{0} / A_{0} \cong Q / R \cong \mathbb{F} s^{\prime} \oplus \bigoplus_{t=k+1}^{\ell} \mathfrak{g}_{0}^{[t]}$, which is a direct sum of ideals of type (a), (b), or (c).

(ii) With notation as in the proof of (i), observe first that $A_{0}^{(1)}=K=$ $\bigoplus_{i=1}^{k}\left(\mathfrak{g}_{0}^{[i]}\right)^{(1)}$. Now let $J$ be a solvable ideal of $A_{0}$. Then $\left[J, A_{0}\right]$ is a solvable ideal of $A_{0}^{(1)}$. Since each nonzero $\left(\mathfrak{g}_{0}^{[i]}\right)^{(1)}$ is nonsolvable, and any proper ideal of $\left(\mathfrak{g}_{0}^{[i]}\right)^{(1)}$ lies in $\mathfrak{Z}\left(\mathfrak{g}_{0}\right)$, we obtain $\left[J, A_{0}\right] \subseteq \mathfrak{Z}\left(\mathfrak{g}_{0}\right)$. Then $\pi_{i}(J)$ is a subalgebra of $\mathfrak{g}_{0}^{[i]}$ satisfying

$$
\left[\pi_{i}(J),\left(\mathfrak{g}_{0}^{[i]}\right)^{(1)}\right] \subseteq \pi_{i}\left(\left[J, A_{0}\right]\right) \subseteq \mathfrak{Z}\left(\mathfrak{g}_{0}\right) \cap \pi_{i}(J) .
$$


It is easy to see that $\mathfrak{g}_{0}^{[i]}$ contains a maximal toral subalgebra $\mathfrak{t}^{[i]}$ such that

(1) $\left(\mathfrak{t}^{[i]}\right)^{\prime}:=\mathfrak{t}^{[i]} \cap\left(\mathfrak{g}_{0}^{[i]}\right)^{(1)}$ is a maximal toral subalgebra of $\left(\mathfrak{g}_{0}^{[i]}\right)^{(1)}$;

(2) any root space of $\left(\mathfrak{g}_{0}^{[i]}\right)^{(1)}$ relative to $\left(\mathfrak{t}^{[i]}\right)^{\prime}$ is one-dimensional;

(3) no root vector of $\left(\mathfrak{g}_{0}^{[i]}\right)^{(1)}$ relative to $\left(\mathfrak{t}^{[i]}\right)^{\prime}$ is central in $\mathfrak{g}_{0}^{[i]}$;

(4) $\mathfrak{g}_{0}^{[i]}=\mathfrak{t}^{[i]}+\left(\mathfrak{g}_{0}^{[i]}\right)^{(1)}$.

Decomposing $\pi_{i}(J)$ into weight spaces relative to $\left(\mathfrak{t}^{[i]}\right)^{\prime}$, one observes readily that $\pi_{i}(J) \subseteq \mathfrak{t}^{[i]}$. From this it follows that $\pi_{i}(J)$ is a solvable ideal of $\mathfrak{g}_{0}^{[i]}$. Then $\pi_{i}(J) \subseteq \mathfrak{Z}\left(\mathfrak{g}_{0}\right)$ by our remarks earlier in the proof. But then $J \subseteq \mathfrak{Z}\left(\mathfrak{g}_{0}\right)$, as desired.

\subsection{The proof of Theorem 1.63 when $2 \leq q \leq r$}

Having established our result in the special case of depth-one Lie algebras, we turn our attention now to graded Lie algebras $\mathfrak{g}=\bigoplus_{i=-q}^{r} \mathfrak{g}_{i}$ with $q \geq 2$ satisfying the requirements of Theorem 1.63. In analyzing such Lie algebras, we will make extensive use of the depth-one graded Lie algebras $\mathcal{B}\left(\mathfrak{g}_{-q}\right)$ and $\mathcal{B}\left(V_{-q+1}\right)$ described in Section 1.4.

Suppose now that $\mathfrak{g}=\bigoplus_{j=-q}^{r} \mathfrak{g}_{j} \quad$ is a graded Lie algebra with $q \geq$ 2 satisfying the assumptions of Theorem 1.63. Replacing $\mathfrak{g}_{-j}$ by $\mathfrak{g}_{-1}^{j}=$ $\left(\operatorname{ad} \mathfrak{g}_{-1}\right)^{j-1} \mathfrak{g}_{-1}$ for $j=2, \ldots, q$ if necessary, we may suppose that $\mathfrak{g}_{-j}=\mathfrak{g}_{-1}^{j}$. Moreover, factoring out the Weisfeiler radical $\mathcal{M}(\mathfrak{g})$ we may assume also that $\mathcal{M}(\mathfrak{g})=0$ (compare (ii) of Proposition 1.8). Neither of these steps alters the local part $\mathfrak{g}_{-1} \oplus \mathfrak{g}_{0} \oplus \mathfrak{g}_{1}$ (hence the assumptions of transitivity (1.2) and irreducibility (1.1) are still intact as are the constraints on $\left.\mathfrak{g}_{0}\right)$. In this section we will suppose that $q \leq r$.

By Lemma 1.41 (b) we know that $\mathfrak{g}_{-q}$ is an irreducible $\mathfrak{g}_{0}$-module. Moreover, if $F=F\left(\mathfrak{g}_{-q}\right)=\bigoplus_{i \geq-1} F_{i}$ and $\mathcal{B}=\mathcal{B}\left(\mathfrak{g}_{-q}\right)=\bigoplus_{i \geq-1} \mathcal{B}_{i}=F / A$, then by Lemma $1.49, \mathcal{B}_{1} \neq 0$. As $\mathcal{B}_{0}=\mathfrak{g}_{0} / A_{0}$, it follows from Lemma 1.71 that all the conditions of Theorem 1.63 are satisfied by the depth-one algebra $\mathcal{B}$. Therefore, we know from Lemma 1.69 that $(\operatorname{ad} b)^{p}-\operatorname{ad} b^{[p]}=0$ on $\mathcal{B}_{-1}$ for all $b \in \mathcal{B}_{0}^{(1)}$, or equivalently $\left((\operatorname{ad} x)^{p}-\operatorname{ad} x^{[p]}\right)\left(\mathfrak{g}_{-q}\right)=0$ for all $x \in \mathfrak{g}_{0}^{(1)}$. But $(\operatorname{ad} x)^{p}-\operatorname{ad} x^{[p]}=q \chi(x)^{p}$ id on $\mathfrak{g}_{-q}$ by Lemma 1.65 (ii), so either $q \equiv 0$ $\bmod p$ or $\chi(x)=0$ for all $x \in \mathfrak{g}_{0}^{(1)}$. If $q \not \equiv 0 \bmod p$, then $\chi(x)=0$, and we have our desired conclusion that the representation of $\mathfrak{g}_{0}^{(1)}$ on $\mathfrak{g}_{-1}$ is restricted. We will assume then that $q \equiv 0 \bmod p$. Since we are also supposing $p \geq 5$, we have $q \geq 5$ (in fact $q>3$ will suffice for our purposes).

Now we let $V_{-q+1}$ be an irreducible submodule of the $\mathfrak{g}_{0}$-module $\mathfrak{g}_{-q+1}$. Applying Lemma 1.54 (which assumes $3<q \leq r$ ), we determine that $\left(\operatorname{ad} V_{-q+1}\right)^{2} \mathfrak{g}_{q-1} \neq 0$ and hence $\mathcal{B}_{1}^{\prime} \neq 0$ for $\mathcal{B}^{\prime}=\mathcal{B}\left(V_{-q+1}\right)$. In view of Lemma 1.69, it follows from this that $(q-1) \chi(x)=0$ for all $x \in \mathfrak{g}_{0}^{(1)}$. 
But we are assuming $q \equiv 0 \bmod p$, so $\chi(x)=0$ for all $x \in \mathfrak{g}_{0}^{(1)}$. As a consequence, we have established

LEMMA 1.73. Let $\mathfrak{g}=\bigoplus_{i=-q}^{r} \mathfrak{g}_{i}$ be a graded Lie algebra satisfying the hypotheses of Theorem 1.63, and assume $q \leq r$. Then the representation of $\mathfrak{g}_{0}^{(1)}=\left[\mathfrak{g}_{0}, \mathfrak{g}_{0}\right]$ on $\mathfrak{g}_{-1}$ is a restricted representation.

\subsection{The proof of Theorem 1.63 when $q>r$}

As in the argument above, in order to show $\mathfrak{g}_{0}^{(1)}$ has a restricted action on $\mathfrak{g}_{-1}$, it suffices to work with the subalgebra $\widehat{\mathfrak{g}}$ of $\mathfrak{g}$ generated by the local part $\mathfrak{g}_{-1} \oplus \mathfrak{g}_{0} \oplus \mathfrak{g}_{1}$ of $\mathfrak{g}$. We may suppose $\widehat{\mathfrak{g}}=\bigoplus_{i=-q^{\prime}}^{r^{\prime}} \widehat{\mathfrak{g}}_{i}$ where $\widehat{\mathfrak{g}}_{-q^{\prime}} \neq 0$ and $\widehat{\mathfrak{g}}_{r^{\prime}} \neq 0, \widehat{\mathfrak{g}}_{0}=\mathfrak{g}_{0}$, and $\widehat{\mathfrak{g}}_{ \pm i}=\left(\mathfrak{g}_{ \pm 1}\right)^{i}$ for $i>0$ (compare (1.38)). Then if we factor out the Weisfeiler radical of $\widehat{\mathfrak{g}}$ (contained in $\sum_{j \leq-2} \widehat{\mathfrak{g}}_{j}$ ), we do not affect the local part of $\widehat{\mathfrak{g}}$ nor do we alter the action of ad $\widehat{\mathfrak{g}}_{0}$ on $\widehat{\mathfrak{g}}_{-1}$ or the transitivity (1.2). Under these assumptions, $\mathfrak{g}$ is 1 -transitive in its negative part by Lemma 1.12. With these reductions, we may suppose that the following holds.

\subsection{General setup}

(i) $\mathfrak{g}=\bigoplus_{i=-q}^{r} \mathfrak{g}_{i}$ is a transitive, irreducible graded Lie algebra generated by its local part $\mathfrak{g}_{-1} \oplus \mathfrak{g}_{0} \oplus \mathfrak{g}_{1}$;

(ii) $\mathcal{M}(\mathfrak{g})=0$;

(iii) $q>r$;

(iv) $\mathfrak{g}$ is 1 -transitive in its negative part (i.e., $\left[\mathfrak{g}_{1}, x\right]=0$ for $x \in \mathfrak{g}_{-}$ implies $x=0$ );

(v) the unique minimal ideal is $\mathcal{J}=\bigoplus_{i=-q}^{s} \mathcal{J}_{i}$;

(vi) $\mathfrak{g}_{0}$ is the sum of ideals satisfying (a)-(c) of Theorem 1.63; and

(vii) $\left[\left[\mathfrak{g}_{-1}, \mathfrak{g}_{1}\right], \mathfrak{g}_{1}\right] \neq 0$.

Our implicit assumption in all subsequent results in Section 1.16 is that (i)-(vii) hold.

LEMma 1.74. We have $\operatorname{Ann}_{\mathfrak{g}_{0}}\left(\mathfrak{g}_{1}\right)=0$ so that $\mathfrak{g}$ is 1-transitive, and $s>0$.

Proof. Suppose that $Q:=\operatorname{Ann}_{\mathfrak{g}_{0}}\left(\mathfrak{g}_{1}\right) \neq 0$. By transitivity (1.2) and irreducibility (1.1), $\left[\mathfrak{g}_{-1}, Q\right]=\mathfrak{g}_{-1}$. Therefore, $\left[\mathfrak{g}_{-1}, \mathfrak{g}_{1}\right]=\left[\left[\mathfrak{g}_{-1}, Q\right], \mathfrak{g}_{1}\right]=$ $\left[\left[\mathfrak{g}_{-1}, \mathfrak{g}_{1}\right], Q\right] \subseteq Q$. But then $\left[\left[\mathfrak{g}_{-1}, \mathfrak{g}_{1}\right], \mathfrak{g}_{1}\right]=0$ contrary to assumption (vii). This gives $Q=0$, which in conjunction with (iv) shows that $\mathfrak{g}$ is 1-transitive. Now if $s=0$, then $\left[\mathcal{J}_{0}, \mathfrak{g}_{1}\right] \subseteq \mathcal{J} \cap \mathfrak{g}_{1}=0$, hence $\mathcal{J}_{0}=0$. Since $0 \neq\left[\mathfrak{g}_{-1}, \mathfrak{g}_{1}\right] \subseteq \mathcal{J}_{0}$, this is impossible. This demonstrates that $s>0$ must hold. 
LEMmA 1.75. $\mathfrak{g}_{r} \cap \mathcal{J}=0$ if and only if $\mathfrak{g}_{r}$ is a trivial $\left[\mathfrak{g}_{-1}, \mathfrak{g}_{1}\right]$-module. In this case, $\left[\mathfrak{g}_{1}, \mathfrak{J}_{r-1}\right]=0$ and $\mathfrak{J}_{r-1}$ is a nontrivial $\left[\mathfrak{g}_{-1}, \mathfrak{g}_{1}\right]$-module.

Proof. From Lemma 1.16 it follows that the unique minimal graded ideal $\mathcal{J}$ of $\mathfrak{g}$ satisfies $\mathcal{J}_{-i}=\mathfrak{g}_{-i}, 1 \leq i \leq q$, and $\mathcal{J}_{j}=\left[\mathfrak{g}_{-1}, \mathcal{J}_{j+1}\right],-q \leq j \leq r-2$. Now if $\mathfrak{g}_{r} \cap \mathcal{J}=0$, then since $\mathfrak{g}_{-1} \subseteq \mathcal{J}$,

$$
\left[\left[\mathfrak{g}_{-1}, \mathfrak{g}_{1}\right], \mathfrak{g}_{r}\right] \subseteq\left[\mathcal{J}_{0}, \mathfrak{g}_{r}\right] \subseteq \mathfrak{g}_{r} \cap \mathcal{J}=0,
$$

so that $\mathfrak{g}_{r}$ is a trivial $\left[\mathfrak{g}_{-1}, \mathfrak{g}_{1}\right]$-module. Conversely, if $\mathfrak{g}_{r}$ is a trivial $\left[\mathfrak{g}_{-1}, \mathfrak{g}_{1}\right]$ module, then $\sum_{i \geq 1}\left(\operatorname{ad} \mathfrak{g}_{-1}\right)^{i} \mathfrak{g}_{r}$ is an ideal of $\mathfrak{g}$ contained in $\mathcal{J}$, which by minimality must equal $\mathcal{J}$. Consequently, $\mathcal{J} \cap \mathfrak{g}_{r}=0$.

Now if $\mathfrak{g}_{r}$ is a trivial $\left[\mathfrak{g}_{-1}, \mathfrak{g}_{1}\right]$-module (equivalently, if $\mathfrak{g}_{r} \cap \mathcal{J}=0$ ), then $\sum_{i \geq 1}\left(\text { ad } \mathfrak{g}_{-1}\right)^{i} \mathfrak{g}_{r}=\mathcal{J}$ as noted above, to force $\mathcal{J}_{r-1}=\left[\mathfrak{g}_{-1}, \mathfrak{g}_{r}\right]$. As a result, $\left[\mathfrak{g}_{1}, \mathfrak{J}_{r-1}\right]=\left[\mathfrak{g}_{1},\left[\mathfrak{g}_{-1}, \mathfrak{g}_{r}\right]\right]=\left[\left[\mathfrak{g}_{1}, \mathfrak{g}_{-1}\right], \mathfrak{g}_{r}\right]+\left[\mathfrak{g}_{-1},\left[\mathfrak{g}_{1}, \mathfrak{g}_{r}\right]\right]=0$. If $\mathcal{J}_{r-1}$ happens to be a trivial $\left[\mathfrak{g}_{-1}, \mathfrak{g}_{1}\right]$-module, then we would have

$$
0=\left[\left[\mathfrak{g}_{-1}, \mathfrak{g}_{1}\right], \mathcal{J}_{r-1}\right]=\left[\left[\mathfrak{g}_{-1}, \mathfrak{g}_{1}\right],\left[\mathfrak{g}_{-1}, \mathfrak{g}_{r}\right]\right]=\left[\left[\left[\mathfrak{g}_{-1}, \mathfrak{g}_{1}\right], \mathfrak{g}_{-1}\right], \mathfrak{g}_{r}\right]=\left[\mathfrak{g}_{-1}, \mathfrak{g}_{r}\right]
$$

by the transitivity (1.2) and irreducibility (1.1) of $\mathfrak{g}$, to contradict transitivity.

We can use the 1-transitivity established in Lemma 1.74 to argue that $\mathfrak{g}_{r}$ annihilates no nonzero element in $\mathfrak{g}_{\leq 0}$. In fact, we can prove a somewhat stronger result. Let $M$ be any graded $\mathfrak{g}_{0}$-submodule of $\mathfrak{g}$ such that $\left[\mathfrak{g}_{1}, M\right]$ $=0$. By 1-transitivity, $M \subseteq \mathfrak{g}_{+}$. Suppose that $[M, x]=0$ for some nonzero $x \in \mathfrak{g}_{-}$. Clearly, $M$ annihilates each of the homogeneous summands $x_{-i}$ $\in \mathfrak{g}_{-i}$ of $x$. For any $j$ such that $x_{-j} \neq 0$, set $N_{-j}:=\left\{y \in \mathfrak{g}_{-j} \mid[M, y]=0\right\}$, which is a nonzero $\mathfrak{g}_{0}$-submodule of $\mathfrak{g}_{-j}$. Then by Section 1.16 (iv),

$$
\left(\operatorname{ad} \mathfrak{g}_{1}\right)^{j-1}\left[N_{-j}, M\right]=\left[\left(\operatorname{ad} \mathfrak{g}_{1}\right)^{j-1} N_{-j}, M\right]=\left[\mathfrak{g}_{-1}, M\right] \neq 0,
$$

contrary to the definition of $N_{-j}$. Consequently, $\operatorname{Ann}_{\mathfrak{g}_{-}}(M)=0$. In view of Lemma 1.75, we have proven

LEMMA 1.76. If $M$ is any graded $\mathfrak{g}_{0}$-submodule of $\mathfrak{g}$ such that $\left[M, \mathfrak{g}_{1}\right]=$ 0 , then $\operatorname{Ann}_{\mathfrak{g}_{-}}(M)=0$. In particular, $\operatorname{Ann}_{\mathfrak{g}_{-}}\left(\mathfrak{g}_{r}\right)=0$. If $\mathfrak{g}_{r}$ is a trivial $\left[\mathfrak{g}_{-1}, \mathfrak{g}_{1}\right]$-module, then $\mathrm{Ann}_{\mathfrak{g}_{-}}\left(\mathcal{J}_{r-1}\right)=0$.

We now want to show

Lemma 1.77. Suppose $M_{j}$ is a $\mathfrak{g}_{0}$-submodule of $\mathfrak{g}_{j}$ with $0<j<q$, such that $\left[\left[\mathfrak{g}_{-1}, \mathfrak{g}_{1}\right], M_{j}\right] \neq 0$ and $\left[\mathfrak{g}_{1}, M_{j}\right]=0$. Then $\left[M_{j},\left[M_{j}, \mathfrak{g}_{-j}\right]\right] \neq 0$. 
Proof. First we observe that

$$
\left[M_{j},\left(\operatorname{ad} \mathfrak{g}_{1}\right)^{q-j-1} \mathfrak{g}_{-q}\right]=\left(\operatorname{ad} \mathfrak{g}_{1}\right)^{q-j-1}\left(\left[M_{j}, \mathfrak{g}_{-q}\right]\right)=\mathfrak{g}_{-1},
$$

thanks to Section 1.16 (iv) and Lemma 1.76. Therefore,

$$
\begin{aligned}
0 \neq\left[\left[\mathfrak{g}_{-1}, \mathfrak{g}_{1}\right], M_{j}\right] & =\left[\left[\left[M_{j},\left(\operatorname{ad} \mathfrak{g}_{1}\right)^{q-j-1} \mathfrak{g}_{-q}\right], \mathfrak{g}_{1}\right], M_{j}\right] \\
& =\left[M_{j},\left[M_{j},\left(\operatorname{ad} \mathfrak{g}_{1}\right)^{q-j} \mathfrak{g}_{-q}\right]\right] \\
& \subseteq\left[M_{j},\left[M_{j}, \mathfrak{g}_{-j}\right]\right] .
\end{aligned}
$$

Lemma 1.78. We have $\left[\mathcal{J}_{s},\left[\mathcal{J}_{s}, \mathfrak{g}_{-s}\right]\right] \neq 0$

Proof. If $\mathfrak{g}_{r} \cap \mathcal{J} \neq 0$ (and hence $s=r$ ), then by Lemma 1.75, $\mathfrak{g}_{r}$ is a nontrivial $\left[\mathfrak{g}_{-1}, \mathfrak{g}_{1}\right]$-module. Since $0<r<q$, we then apply Lemma 1.77 with $M_{j}=\mathfrak{g}_{r}$ to conclude that $\left[\mathfrak{g}_{r},\left[\mathfrak{g}_{r}, \mathfrak{g}_{-r}\right]\right] \neq 0$. On the other hand, if $\mathfrak{g}_{r} \cap \mathcal{J}=0$, then by Lemma 1.75, $\left[\mathfrak{g}_{1}, \mathcal{J}_{r-1}\right]=0$ and $\mathcal{J}_{r-1}$ is a nontrivial $\left[\mathfrak{g}_{-1}, \mathfrak{g}_{1}\right]$-module. Furthermore $q>r-1=s$ in this case, and $s>0$ by Lemma 1.74, so Lemma 1.77 applies with $M_{j}=\mathcal{J}_{r-1}=\mathcal{J}_{s}$. Thus, we deduce that in any event $\left[\mathcal{J}_{s},\left[\mathcal{J}_{s}, \mathfrak{g}_{-s}\right]\right] \neq 0$.

Recall we are assuming that $\mathfrak{g}$ is generated by its local part. Because $\left[\mathfrak{g}_{1}, \mathcal{J}_{s}\right] \subseteq \mathcal{J}_{s+1}=0$, it follows that any $\mathfrak{g}_{0}$-submodule $R_{s}$ of $\mathcal{J}_{s}$ generates an ideal

$$
J:=\sum_{i \geq 0}\left(\operatorname{ad} \mathfrak{g}_{-1}\right)^{i} R_{s}
$$

which is contained in $\mathcal{J}$. By the minimality of $\mathcal{J}$, it must be that $J=\mathcal{J}$, so that $\mathcal{J}_{s}=R_{s}$; i.e., $\mathcal{J}_{s}$ is $\mathfrak{g}_{0}$-irreducible. Thus, in view of Lemma 1.78 , we can apply Lemma 1.69 to conclude that the representation of $\mathcal{B}\left(\mathcal{J}_{s}\right)_{0}^{(1)}$ on $\mathcal{B}\left(\mathcal{J}_{s}\right)_{-1}$ is a restricted representation. Since we are also assuming that $\mathfrak{g}$ is transitive, the $\mathfrak{g}_{0}$-module $\mathfrak{g}_{1}$ is isomorphic to a $\mathfrak{g}_{0}$-submodule of Hom $\left(\mathfrak{g}_{-1}, \mathfrak{g}_{0}\right) \cong \mathfrak{g}_{-1}^{*} \otimes_{\mathbb{F}} \mathfrak{g}_{0}$. From this it follows that for every $x \in \mathfrak{g}_{0}^{(1)}$, the endomorphism $(\operatorname{ad} x)^{p}-$ ad $x^{[p]}$ acts on $\mathfrak{g}_{1}$ as $-\chi(x)^{p}$ id. But then $(\operatorname{ad} x)^{p}-\operatorname{ad} x^{[p]}=-s \chi(x)^{p}$ id on $\mathfrak{g}_{s}=\left(\mathfrak{g}_{1}\right)^{s}$. As in the discussion of Section 1.14 leading to the proof of Lemma 1.71, either the representation of $\mathfrak{g}_{0}^{(1)}$ on $\mathfrak{g}_{-1}$ is restricted, or $s$ is a multiple of $p$. In summary, we have

Lemma 1.79. The $\mathfrak{g}_{0}$-module $\mathcal{J}_{s}$ is irreducible. Furthermore, either the representation of $\mathfrak{g}_{0}^{(1)}$ on $\mathfrak{g}_{-1}$ is restricted, or $p \mid s$.

Thus, in what follows, we may assume $s$ is a multiple of $p$ so that $s>3$.

To complete the proof of Theorem 1.63, we will show that for any nonzero irreducible $\mathfrak{g}_{0}$-submodule $V_{s-1}$ of $\mathcal{J}_{s-1}$, the condition $\left[V_{s-1},\left[V_{s-1}, \mathfrak{g}_{-s+1}\right]\right] \neq 0$ must hold. 
Lemma 1.80. $\left[\mathcal{J}_{s-1}, \mathfrak{g}_{1}\right]=\mathcal{J}_{s}$, and $\left[V_{s-1}, \mathfrak{g}_{-s+1}\right] \neq 0$ for any nonzero $\mathfrak{g}_{0}$-submodule $V_{s-1}$ of $\mathcal{J}_{s-1}$.

Proof. Let $V_{s-1}$ be a nonzero $\mathfrak{g}_{0}$-submodule of $\mathcal{J}_{s-1}$, and suppose initially that $\left[V_{s-1}, \mathfrak{g}_{1}\right]=0$. Then, since $\mathfrak{g}$ is assumed to be generated by its local part, it would follow that $\sum_{j \geq 0}\left(\text { ad } \mathfrak{g}_{-1}\right)^{j} V_{s-1}$ is an ideal of $\mathfrak{g}$ properly contained in $\mathcal{J}$; a contradiction to the minimality of $\mathcal{J}$.

Consequently, $0 \neq\left[V_{s-1}, \mathfrak{g}_{1}\right] \subseteq \mathcal{J}_{s}$. By Lemma $1.79, \mathcal{J}_{s}$ is $\mathfrak{g}_{0}$-irreducible, so that $\left[V_{s-1}, \mathfrak{g}_{1}\right]=\mathcal{J}_{s}$. In particular, $\left[\mathcal{J}_{s-1}, \mathfrak{g}_{1}\right]=\mathcal{J}_{s}$. For the second half of the lemma, we may suppose that $\left[V_{s-1}, \mathcal{J}_{-s+1}\right]=0$. Then

$$
0=\left[\mathcal{J}_{s-2},\left[V_{s-1}, \mathcal{J}_{-s+1}\right]\right]=\left[V_{s-1},\left[\mathcal{J}_{s-2}, \mathcal{J}_{-s+1}\right]\right]
$$

since we are assuming that $s>3$, so that $\left[\mathcal{J}_{s-2}, V_{s-1}\right]=0$. If $\left[\mathcal{J}_{s-2}, \mathcal{J}_{-s+1}\right] \neq$ 0 , it is the whole of $\mathfrak{g}_{-1}$ by irreducibility (1.1). But then $\left[V_{s-1}, \mathfrak{g}_{-1}\right]=0$ contradicting transitivity (1.2). This shows that $\left[\mathcal{J}_{s-2}, \mathcal{J}_{-s+1}\right]=0$. Therefore, by our assumption that $\left[V_{s-1}, \mathcal{J}_{-s+1}\right]=0$, we have

$$
\begin{aligned}
0=\left[\mathcal{J}_{s-2}, \mathcal{J}_{-s+1}\right] \supseteq\left[\left[\mathfrak{g}_{-1}, V_{s-1}\right], \mathcal{J}_{-s+1}\right] & =\left[\left[\mathfrak{g}_{-1}, \mathcal{J}_{-s+1}\right], V_{s-1}\right] \\
& =\left[\mathcal{J}_{-s}, V_{s-1}\right] .
\end{aligned}
$$

Since $\mathfrak{g}_{-1} \subset \mathcal{J}$ and $\mathfrak{g}_{-s}=\left(\mathfrak{g}_{-1}\right)^{s}$, we have $\mathcal{J}_{-s}=\mathfrak{g}_{-s}$ forcing $\left[\mathfrak{g}_{-s}, V_{s-1}\right]=0$. We have already established that $\left[V_{s-1}, \mathfrak{g}_{1}\right]=\mathcal{J}_{s}$. Consequently,

$$
\begin{aligned}
0=\left[V_{s-1}, \mathcal{J}_{-s+1}\right] \supseteq\left[V_{s-1},\left[\mathcal{J}_{-s}, \mathfrak{g}_{1}\right]\right] & =\left[V_{s-1},\left[\mathfrak{g}_{-s}, \mathfrak{g}_{1}\right]\right] \\
& =\left[\mathfrak{g}_{-s},\left[V_{s-1}, \mathfrak{g}_{1}\right]\right]=\left[\mathfrak{g}_{-s}, \mathcal{J}_{s}\right]
\end{aligned}
$$

to contradict Lemma 1.78. Thus, $\left[V_{s-1}, \mathcal{J}_{-s+1}\right] \neq 0$, as claimed.

Next we show how

$$
\left[V_{s-1},\left[V_{s-1}, \mathfrak{g}_{-s+1}\right]\right]=0
$$

for a nonzero $\mathfrak{g}_{0}$-submodule of $V_{s-1}$ of $\mathcal{J}_{s-1}$ leads to a contradiction. If (1.81) holds, then

$$
W:=\left\{\operatorname{ad}_{\mathfrak{g}_{s-2}}[v, u] \mid v \in V_{s-1}, u \in \mathfrak{g}_{-s+1}\right\}
$$

is a weakly closed subset of End $\left(\mathfrak{g}_{s-2}\right)$ by Lemma 1.48 (i).

First we assume for all $v \in V_{s-1}, u \in \mathfrak{g}_{-s+1}$ that

$$
(\operatorname{ad} v)^{3}(\operatorname{ad} u)^{3} \mathfrak{g}_{s-2}=0 .
$$

Then $0=(\operatorname{ad} v)^{3}(\operatorname{ad} u)^{3} \mathfrak{g}_{s-2}=6(\operatorname{ad}[u, v])^{3} \mathfrak{g}_{s-2}$, hence $W$ consists of nilpotent transformations. The associative subalgebra of End $\left(\mathfrak{g}_{s-2}\right)$ generated by $W$ must act nilpotently on $\mathfrak{g}_{s-2}$ by Lemma 1.48 (ii), so that

$$
Q_{s-2}:=\operatorname{Ann}_{\mathfrak{g}_{s-2}}\left(\left[V_{s-1}, \mathfrak{g}_{-s+1}\right]\right) \neq 0
$$


If $\left[Q_{s-2}, \mathfrak{g}_{-s+1}\right] \neq 0$, then $\left[Q_{s-2}, \mathfrak{g}_{-s+1}\right]=\mathfrak{g}_{-1}$ by irreducibility (1.1), and since we are assuming $s \geq 5$ (and hence $s-2+s-1>s+1 \geq r$ ), we would have

$$
\left[V_{s-1}, \mathfrak{g}_{-1}\right]=\left[V_{s-1},\left[Q_{s-2}, \mathfrak{g}_{-s+1}\right]\right]=\left[Q_{s-2},\left[V_{s-1}, \mathfrak{g}_{-s+1}\right]\right]=0
$$

by the definition of $Q_{s-2}$. Then transitivity (1.2) would force $V_{s-1}=$ 0 , contrary to hypothesis. Thus, we can conclude that $\left[Q_{s-2}, \mathfrak{g}_{-s+1}\right]=$ 0 . As $\mathfrak{J}_{2 s-4}=0$ (because $s \geq 5$ ), we have $0=\left[\mathcal{J}_{s-2},\left[Q_{s-2}, \mathfrak{g}_{-s+1}\right]\right]=$ $\left[Q_{s-2},\left[\mathcal{J}_{s-2}, \mathfrak{g}_{-s+1}\right]\right]$. Now $\left[\mathcal{J}_{s-2}, \mathfrak{g}_{-s+1}\right]$ equals 0 or $\mathfrak{g}_{-1}$, and when it is nonzero we have

$$
0=\left[Q_{s-2},\left[\mathcal{J}_{s-2}, \mathfrak{g}_{-s+1}\right]\right]=\left[Q_{s-2}, \mathfrak{g}_{-1}\right]
$$

contradicting the transitivity of $\mathfrak{g}$. Thus, $\left[\mathcal{J}_{s-2}, \mathfrak{g}_{-s+1}\right]=0$, and as a result,

$$
0=\left[V_{s-1},\left[\mathcal{J}_{s-2}, \mathfrak{g}_{-s+1}\right]\right]=\left[\mathcal{J}_{s-2},\left[V_{s-1}, \mathfrak{g}_{-s+1}\right]\right] .
$$

Hence, $\left[V_{s-1}, \mathfrak{g}_{-s+1}\right] \subseteq \operatorname{Ann}_{\mathfrak{g}_{0}}\left(\mathcal{J}_{s-2}\right) \cap \operatorname{Ann}_{\mathfrak{g}_{0}}\left(V_{s-1}\right)$, which must be zero by Lemma 1.50. But this contradicts Lemma 1.80.

Thus, we have proven that if (1.81) holds, then (1.82) cannot happen. In other words, there exist $v \in V_{s-1}$ and $u \in \mathfrak{g}_{-s+1}$ such that $(\operatorname{ad} v)^{3}(\operatorname{ad} u)^{3} \mathfrak{g}_{s-2} \neq 0$. Then $U_{s-2}:=\left(\operatorname{ad} V_{s-1}\right)^{3} \mathfrak{g}_{-2 s+1}$ is a nonzero $\mathfrak{g}_{0^{-}}$ submodule of $\mathcal{J}_{s-2}$. Assuming $\left[\mathfrak{g}_{1}, U_{s-2}\right]=\left[\mathfrak{g}_{2}, U_{s-2}\right]=0$ would imply that

$$
\sum_{j \geq 0}\left(\operatorname{ad} \mathfrak{g}_{-1}\right)^{j} U_{s-2}
$$

is an ideal of $\mathfrak{g}$ properly contained in $\mathcal{J}$, a contradiction. When $\left[\mathfrak{g}_{1}, U_{s-2}\right] \neq 0$, then

$$
0 \neq\left[\mathfrak{g}_{1}, U_{s-2}\right]=\left[\mathfrak{g}_{1},\left(\operatorname{ad} V_{s-1}\right)^{3} \mathfrak{g}_{-2 s+1}\right] \subseteq\left(\operatorname{ad} V_{s-1}\right)^{2} \mathfrak{g}_{-s+1} .
$$

On the other hand, when $\left[\mathfrak{g}_{2}, U_{s-2}\right] \neq 0$, then

$$
0 \neq\left[\mathfrak{g}_{2}, U_{s-2}\right] \subseteq\left(\operatorname{ad} V_{s-1}\right)^{3} \mathfrak{g}_{-2 s+3}
$$

In this case, transitivity (1.2) gives

$$
0 \neq\left[\mathfrak{g}_{-1},\left(\operatorname{ad} V_{s-1}\right)^{3} \mathfrak{g}_{-2 s+3}\right] \subseteq\left(\operatorname{ad} V_{s-1}\right)^{2} \mathfrak{g}_{-s+1} .
$$

Consequently, $\left(\text { ad } V_{s-1}\right)^{2} \mathfrak{g}_{s+1} \neq 0$ must hold, and that in conjunction with (1.44), allows us to conclude the following:

LEMmA 1.83. $\left[V_{s-1},\left[V_{s-1}, \mathfrak{g}_{-s+1}\right]\right] \neq 0$ for any nonzero $\mathfrak{g}_{0}$-submodule $V_{s-1}$ of $\mathcal{J}_{s-1}$. When $V_{s-1}$ is irreducible, then for $\mathcal{B}^{\prime}=\mathcal{B}\left(V_{s-1}\right)=\bigoplus \mathcal{B}_{i}^{\prime}$ we have $\mathcal{B}_{1}^{\prime} \neq 0$, and the representation of $\left(\mathcal{B}_{0}^{\prime}\right)^{(1)}$ on $\mathcal{B}_{-1}^{\prime}=V_{s-1}$ is restricted. 
As before, knowing that $\mathcal{B}_{1} \neq 0$ for $\mathcal{B}=\mathcal{B}\left(\mathcal{J}_{s}\right)$ and $\mathcal{B}_{1}^{\prime} \neq 0$ for $\mathcal{B}^{\prime}=$ $\mathcal{B}\left(V_{s-1}\right)$ forces the representation of $\mathfrak{g}_{0}^{(1)}$ on $\mathfrak{g}_{-1}$ to be restricted. Thus, we may draw the conclusion:

Lemma 1.84. Let $\mathfrak{g}=\bigoplus_{i=-q}^{r} \mathfrak{g}_{i}$ be a graded Lie algebra satisfying the hypotheses of Theorem 1.63, and assume that $q>r$ and $\left[\left[\mathfrak{g}_{-1}, \mathfrak{g}_{1}\right], \mathfrak{g}_{1}\right] \neq 0$. Then the representation of $\mathfrak{g}_{0}^{(1)}$ on $\mathfrak{g}_{-1}$ is a restricted representation.

1.85. Combining Lemmas $1.69,1.71,1.73$, and 1.84 completes the proof of Theorem 1.63.

\subsection{The assumption $\left[\left[\mathfrak{g}_{-1}, \mathfrak{g}_{1}\right], \mathfrak{g}_{1}\right] \neq 0$ in Theorem $\mathbf{1 . 6 3}$}

The assumption that $\left[\left[\mathfrak{g}_{-1}, \mathfrak{g}_{1}\right], \mathfrak{g}_{1}\right] \neq 0$ in the statement of Theorem 1.63 cannot be dropped, as the following example illustrates.

Let $\mathfrak{K}$ be a classical simple Lie algebra, and let $\mathfrak{M}$ be an irreducible nonrestricted $\mathfrak{K}$-module with basis $x_{1}, \ldots, x_{m}$. Clearly, $\mathfrak{K}$ acts as homogeneous derivations of degree zero on the graded truncated polynomial algebra

$$
\mathfrak{A}:=\mathbb{F}\left[x_{1}, \ldots, x_{m}\right] /\left\langle x_{1}^{p}, \ldots, x_{m}^{p}\right\rangle, \quad \operatorname{deg} x_{i}=1 .
$$

Note that $\mathfrak{A}=\bigoplus_{i=0}^{r} \mathfrak{A}_{i}$, where $r=m(p-1)$ and $\mathfrak{A}_{0}=\mathbb{F} 1$. Let $\mathfrak{D}$ denote the linear span of the partial derivatives $\partial / \partial x_{1}, \ldots, \partial / \partial x_{m}$ of $\mathfrak{A}$.

We regard $\mathfrak{K}$ as a subalgebra of the derivation algebra $\operatorname{Der}(\mathfrak{A})$. Clearly, $[\mathfrak{K}, \mathfrak{D}] \subseteq \mathfrak{D}$ and $\mathfrak{D}^{(1)}=0$. It follows that $\mathcal{K}:=\mathfrak{K} \oplus \mathfrak{D}$ is a Lie subalgebra of $\operatorname{Der}(\mathfrak{A})$, and $\mathfrak{D}$ is an abelian ideal of $\mathcal{K}$. The pairing $\mathfrak{D} \times \mathfrak{M} \rightarrow \mathbb{F}$, $(d, x) \mapsto d(x)$, is nondegenerate and $\mathfrak{K}$-invariant. Hence, $\mathfrak{D} \cong \mathfrak{M}^{*}$, showing that the $\mathfrak{K}$-module $\mathfrak{D}$ is irreducible and nonrestricted.

Now let $\mathfrak{L}$ be another classical simple Lie algebra. Then $\mathfrak{L} \otimes \mathfrak{A}$ carries a natural Lie algebra structure given by $\left[l \otimes f, l^{\prime} \otimes f^{\prime}\right]=\left[l, l^{\prime}\right] \otimes f f^{\prime}$ for all $l, l^{\prime} \in \mathfrak{L}$ and $f, f^{\prime} \in \mathfrak{A}$. Note that $\operatorname{id}_{\mathfrak{L}} \otimes \mathcal{K}$ is a subalgebra of the derivation algebra of $\mathfrak{L} \otimes \mathfrak{A}$. As a consequence, we may regard

$$
\mathfrak{g}:=(\mathfrak{L} \otimes \mathfrak{A}) \oplus\left(\mathrm{id}_{\mathfrak{L}} \otimes \mathcal{K}\right)
$$

as a semidirect product of Lie algebras. Put

$$
\begin{aligned}
& \mathfrak{g}_{-i}:=\mathfrak{L} \otimes \mathfrak{A}_{i}, \quad 1 \leq i \leq r, \\
& \mathfrak{g}_{0}:=\left(\mathfrak{L} \otimes \mathfrak{A}_{0}\right) \oplus\left(\operatorname{id}_{\mathfrak{L}} \otimes \mathfrak{K}\right), \\
& \mathfrak{g}_{1}:=\operatorname{id}_{\mathfrak{L}} \otimes \mathfrak{D} .
\end{aligned}
$$

Then $\mathfrak{g}=\bigoplus_{i=-r}^{1} \mathfrak{g}_{i}$ and $\left[\mathfrak{g}_{i}, \mathfrak{g}_{j}\right] \subseteq \mathfrak{g}_{i+j}$ for all $i, j$, so that (1.86) defines a grading of $\mathfrak{g}$. It is straightforward to see that $\mathfrak{g}_{0} \cong \mathfrak{L} \oplus \mathfrak{K}$ is classical reductive, $\mathfrak{g}_{-1}=\mathfrak{L} \otimes \mathfrak{M}$ is an irreducible nonrestricted $\mathfrak{g}_{0}$-module, and $\mathfrak{g}_{1} \cong \mathfrak{M}^{*}$ as $\mathfrak{K}$-modules. Moreover, $\mathfrak{g}$ is transitive, and

$$
\left[\left[\mathfrak{g}_{-1}, \mathfrak{g}_{1}\right], \mathfrak{g}_{1}\right] \subseteq\left[\mathfrak{L} \otimes \mathfrak{A}_{0}, \operatorname{id}_{\mathfrak{L}} \otimes \mathfrak{D}\right]=0 .
$$


Transitive gradings similar to the one above were first described by Weisfeiler who termed them degenerate (see $[\mathbf{W}]$ ). They arise quite frequently in the classification theory of simple Lie algebras. 



\section{CHAPTER 2}

\section{Simple Lie Algebras and Algebraic Groups}

\subsection{Introduction}

In finite characteristics, there are simple finite-dimensional graded Lie algebras which resemble those in zero characteristic and others which do not. The former are referred to as "classical", as they are obtained from their characteristic zero counterparts by reducing a Chevalley basis modulo $p$ and factoring out the center as necessary. The latter are the Cartan type Lie algebras, and in characteristic 5, the Melikyan Lie algebras. The Cartan type and Melikyan Lie algebras possess a grading in which the zero component $\mathfrak{g}_{0}$ is isomorphic to one of the linear Lie algebras $\mathfrak{s l}_{m}$ or $\mathfrak{g l}_{m}$ or to one of the symplectic Lie algebras $\mathfrak{s p}_{2 m}$ or $\mathfrak{c s p}_{2 m}$. (The algebra $\mathfrak{c s p}_{2 m}$ is a central extension of $\mathfrak{s p}_{2 m}$ by a one-dimensional center.) The gradation spaces $\mathfrak{g}_{j}$ are modules for $\mathfrak{g}_{0}$; indeed, in each case, $\mathfrak{g}_{-1}$ is the natural module (or its dual module) for $\mathfrak{s l}_{m}, \mathfrak{g l}_{m}, \mathfrak{s p}_{2 m}$, or $\mathfrak{c s p}_{2 m}$. In this chapter, we gather information about the classical, Cartan type, and Melikyan Lie algebras. Our purpose is two-fold. On one hand, the focus of this monograph is a classification theorem, and these are the Lie algebras which will appear in the theorem's conclusion. On the other, a lot of our discussion will consist of calculations involving these Lie algebras, and we will include here enough facts about them to enable the reader to follow these computations without too much difficulty, we hope.

\subsection{General information about the classical Lie algebras}

The well-established structure theory of finite-dimensional simple Lie algebras $\dot{\mathfrak{g}}$ over the field $\mathbb{C}$ of complex numbers begins with a Cartan subalgebra $\dot{\mathfrak{h}}$ (equivalently, a maximal toral subalgebra) and the root space decomposition $\dot{\mathfrak{g}}=\dot{\mathfrak{h}} \oplus \bigoplus_{\alpha \in \Phi} \dot{\mathfrak{g}}^{\alpha}$ relative to $\dot{\mathfrak{h}}$. The root spaces $\dot{\mathfrak{g}}^{\alpha}=\{x \in$ $\dot{\mathfrak{g}} \mid[h, x]=\alpha(h) x$ for all $h \in \dot{\mathfrak{h}}\}$ are all one-dimensional. Because the Killing form $\kappa(x, y)=\operatorname{tr}(\operatorname{ad} x \operatorname{ad} y)$ is nondegenerate and invariant, $\dot{\mathfrak{g}}^{\alpha}$ and $\dot{\mathfrak{g}}^{-\alpha}$ are nondegenerately paired, and the form restricted to $\dot{\mathfrak{h}}$ is nondegenerate. Thus, for any $\mu \in \dot{\mathfrak{h}}^{*}$, there exists a unique element $t_{\mu} \in \dot{\mathfrak{h}}$ such that $\mu(h)=\kappa\left(h, t_{\mu}\right)$ for all $h \in \dot{\mathfrak{h}}$. This allows the form to be transferred to the dual space $\dot{\mathfrak{h}}^{*}$, where $(\mu, \nu):=\kappa\left(t_{\mu}, t_{\nu}\right)$. In particular, this form restricted to the real span of the roots is positive definite. For $\alpha, \beta \in \Phi$, we set 


$$
\langle\beta, \alpha\rangle:=\frac{2(\beta, \alpha)}{(\alpha, \alpha)} \quad \text { and } \quad h_{\alpha}=\frac{2 t_{\alpha}}{(\alpha, \alpha)}
$$

There is a basis of $\dot{\mathfrak{h}}^{*}$ of simple roots $\Delta=\left\{\alpha_{1}, \ldots, \alpha_{m}\right\} \subseteq \Phi$, and every root is a nonnegative or nonpositive integral linear combination of them. The positive roots ( $\alpha>0$ for short) are the former, and the negative roots $(\alpha<0)$ are the latter. For brevity we will write $h_{i}=h_{\alpha_{i}}$ for $i=1, \ldots, m$.

There is a second distinguished basis for $\dot{\mathfrak{h}}^{*}$, namely the basis $\left\{\varpi_{1}, \ldots\right.$, $\left.\varpi_{m}\right\}$ of fundamental weights. They are dual to the elements $h_{i}$, and so satisfy

$$
\varpi_{i}\left(h_{j}\right)=\left\langle\varpi_{i}, \alpha_{j}\right\rangle=\delta_{i, j} .
$$

The Cartan matrix $A=\left(\left\langle\alpha_{i}, \alpha_{j}\right\rangle\right)$ gives the transition between these two bases: $\alpha_{i}=\sum_{j=1}^{m} A_{i, j} \varpi_{j}, \quad A_{i, j}=\left\langle\alpha_{i}, \alpha_{j}\right\rangle$ for $1 \leq i, j \leq m$. It also encodes all the structural information about $\dot{\mathfrak{g}}$.

THEOREM 2.3. Let $\dot{\mathfrak{g}}$ be a finite-dimensional complex simple Lie algebra. Then there is a basis $\left\{e_{\alpha} \mid \alpha \in \Phi\right\} \cup\left\{h_{i} \mid i=1, \ldots, m\right\}$ of $\dot{\mathfrak{g}}$ such that

(i) $\left[h_{i}, h_{j}\right]=0, \quad 1 \leq i, j \leq m$;

(ii) $\left[h_{i}, e_{\alpha}\right]=\left\langle\alpha, \alpha_{i}\right\rangle e_{\alpha}=\alpha\left(h_{i}\right) e_{\alpha} \quad 1 \leq i \leq m, \quad \alpha \in \Phi$;

(iii) $\left[e_{\alpha}, e_{-\alpha}\right]=h_{\alpha}=\sum_{j=1}^{m}\left\langle\varpi_{j}, \alpha\right\rangle h_{j}$, which is an integral linear combination of $h_{1}, \ldots, h_{m}$;

(iv) If $\beta \neq \pm \alpha$, and $\beta-d \alpha, \ldots, \beta+u \alpha$ is the $\alpha$-string through $\beta$, then

$$
\left[e_{\alpha}, e_{\beta}\right]= \begin{cases}0 & \text { if } u=0 \\ \pm(d+u) e_{\alpha+\beta} & \text { if } u \neq 0,\end{cases}
$$

where the integers $d, u$ satisfy $d-u=\langle\beta, \alpha\rangle$.

Such a basis is referred to as a Chevalley basis. The $\mathbb{Z}$-span $\dot{\mathfrak{g}}_{\mathbb{Z}}$ of a Chevalley basis is a $\mathbb{Z}$-subalgebra of $\dot{\mathfrak{g}}$. If $\mathbb{F}$ is any field, then $\mathfrak{g}=\mathfrak{g} \mathbb{F}:=\mathbb{F} \otimes_{\mathbb{Z}} \dot{\mathfrak{g}}_{\mathbb{Z}}$ is a Lie algebra over $\mathbb{F}$. It has a basis as in Theorem 2.3 , but with the structure constants reduced modulo $p$. If $\mathbb{F}$ has characteristic $p>3$, the Lie algebra $\mathfrak{g}$ is simple in all instances except when $\Phi$ is a root system of type $\mathrm{A}_{m}$ for $p \mid(m+1)$. In that particular case, $\mathfrak{g}$ is isomorphic to $\mathfrak{s l}_{m+1}$, and $\mathfrak{g}$ has a one-dimensional center spanned by the identity matrix $I=h_{1}+2 h_{2}+\cdots+m h_{m}$ (where $h_{i}$ is identified with the difference of matrix units, $\left.h_{i}=E_{i, i}-E_{i+1, i+1}\right)$. Then $\mathfrak{g} / \mathbb{F} I \cong \mathfrak{p s l}_{m+1}$ is simple. The simple Lie algebras obtained by the process of reducing a Chevalley basis modulo $p$ (and factoring out the center if necessary) are called the classical simple Lie algebras. The classical simple Lie algebras along with $\mathfrak{s l}_{m+1}, \mathfrak{g l}_{m+1}$, or $\mathfrak{p g l}_{m+1}$ where $p \mid(m+1)$ and direct sums of these algebras are known 
under the general rubric of classical Lie algebras. A classical reductive Lie algebra modulo its center is a classical Lie algebra. Assumption (a) of the Recognition Theorem asserts that $\mathfrak{g}_{0}$ is classical reductive.

Each classical simple Lie algebra is equipped with a $[p]$-operation relative to which the Lie algebra is restricted:

$$
e_{\alpha}^{[p]}=0 \quad \text { for } \quad \alpha \in \Phi, \quad \text { and } \quad h_{i}^{[p]}=h_{i} \quad \text { for } \quad i=1, \ldots, m .
$$

Mills and Seligman [MS] found a simple set of axioms which characterize direct sums of these Lie algebras.

TheOREM 2.5. A Lie algebra $L$ over a field $\mathbb{F}$ of characteristic $p>3$ is a direct sum of classical simple Lie algebras if and only if

(i) $[L, L]=L$;

(ii) The center of $L$ is 0 ;

(iii) $L$ has an abelian Cartan subalgebra $H$ such that

(a) $L=\bigoplus_{\alpha \in H^{*}} L^{\alpha}$, where

$L^{\alpha}=\{x \in L \mid[h, x]=\alpha(h) x$ for all $h \in H\}$;

(b) If $L^{\alpha} \neq 0$ and $\alpha \neq 0$, (i.e., if $\alpha$ is a root), then $\left[L^{\alpha}, L^{-\alpha}\right]$ is one-dimensional;

(c) If $\alpha$ and $\beta$ are roots, then not all $\beta+k \alpha$ for $k=0,1, \ldots, p-1$, are roots.

Remark 2.6. According to [S, Chap. IV, Thm. 1.2 and Chap. VI, Sec. 2], the Cartan subalgebras of a classical simple Lie algebra $\mathfrak{g}$ over an algebraically closed field of characteristic $p>3$ are all conjugate under the automorphism group of $\mathfrak{g}$. Combining that result with Theorem 2.5, we see that any Cartan subalgebra $\mathfrak{h}$ of $\mathfrak{g}$ is abelian and that the elements of $\mathfrak{h}$ are ad-semisimple on $\mathfrak{g}$. Hence $\mathfrak{h}$ is a toral subalgebra of $\mathfrak{g}$ (in fact, a maximal toral subalgebra of $\mathfrak{g}$ ). As the centralizer of a maximal toral subalgebra of a restricted Lie algebra is a Cartan subalgebra, and every Cartan subalgebra is such a centralizer (for example, [SF, Thm. 4.1]), it follows that the maximal toral subalgebras of a classical simple Lie algebra are precisely the Cartan subalgebras. We will use the two notions interchangeably when working with a classical simple Lie algebra or with one of the algebras $\mathfrak{s l}_{m+1}, \mathfrak{g l}_{m+1}$, or $\mathfrak{p g l}_{m+1}$ with $p \mid(m+1)$, where the same result applies. All maximal toral subalgebras of a classical simple Lie algebra are conjugate - a result which follows from the above considerations for $p>3$, but is known to hold for all characteristics by [H1, Cor. 13.5].

The derivations of a classical simple Lie algebra are inner except when $\mathfrak{g}=\mathfrak{p s l}_{m+1}$. More specifically, we have 
Lemma 2.7. Suppose that $\mathfrak{g}$ is either a classical simple Lie algebra or $\mathfrak{p g l}_{m+1}$ with $p \mid(m+1)$. Then $\operatorname{Der}(\mathfrak{g}) \cong$ ad $\mathfrak{g}$ unless $\mathfrak{g}=\mathfrak{p s l}_{m+1}$ with $p \mid(m+1)$ in which case $\operatorname{Der}(\mathfrak{g}) \cong \mathfrak{p g l}_{m+1}$.

Proof. First suppose that $\mathfrak{g}$ is simple. We have $\mathfrak{g}=\mathfrak{h} \oplus \bigoplus_{\alpha \in \Phi} \mathfrak{g}^{\alpha}$ where $\mathfrak{h}$ is a Cartan subalgebra of $\mathfrak{g}$. Since ad $\mathfrak{h}$ acts diagonalizably on $\mathfrak{g}$, it also acts diagonalizably on $\operatorname{End}(\mathfrak{g}) \cong \mathfrak{g} \otimes \mathfrak{g}^{*}$. It follows that ad $\mathfrak{h}$ acts diagonalizably on $\operatorname{Der}(\mathfrak{g}) \subset \operatorname{End}(\mathfrak{g})$. Let $\widetilde{\mathfrak{h}}$ denote the centralizer of ad $\mathfrak{h}$ in $\operatorname{Der}(\mathfrak{g})$. If $D \in \operatorname{Der}(\mathfrak{g})$ is a weight vector for $\mathfrak{h}$ corresponding to a nonzero weight $\mu \in \mathfrak{h}^{*}$, then $\mu(h) \neq 0$ for some $h \in \mathfrak{h}$, forcing

$$
D=\mu(h)^{-1}[\operatorname{ad} h, D]=-\mu(h)^{-1} \operatorname{ad} D(h) \in \operatorname{ad} \mathfrak{g} .
$$

As a consequence, $\operatorname{Der}(\mathfrak{g})=\widetilde{\mathfrak{h}}+$ ad $\mathfrak{g}$. If $D \in \widetilde{\mathfrak{h}}$, then $D\left(\mathfrak{g}^{\alpha}\right) \subseteq \mathfrak{g}^{\alpha}$ for all $\alpha \in \Phi$, as all root spaces of $\mathfrak{g}$ relative to $\mathfrak{h}$ are one-dimensional. For each $\alpha_{i} \in \Delta$, choose nonzero vectors $e_{i} \in \mathfrak{g}^{\alpha_{i}}$ and $f_{i} \in \mathfrak{g}^{-\alpha_{i}}$. Since $D$ commutes with ad $\left[e_{i}, f_{i}\right] \in$ ad $\mathfrak{h} \backslash\{0\}$, it must be that $D\left(e_{i}\right)=\lambda_{i} e_{i}$ and $D\left(f_{i}\right)=-\lambda_{i} f_{i}$ for some $\lambda_{i} \in \mathbb{F}$, where $1 \leq i \leq m$. Since in the present case the Lie algebra $\mathfrak{g}$ is generated by the $e_{i}$ 's and $f_{i}$ 's, we may conclude that $\operatorname{dim} \widetilde{\mathfrak{h}} \leq m$.

Let $\bar{A}$ denote the matrix with entries in $\mathbb{F}_{p}$ obtained by reducing the entries of the Cartan matrix $A$ of $\mathfrak{g}$ modulo $p$. If $\mathfrak{g} \neq \mathfrak{p s l}_{m+1}$ with $p \mid(m+1)$, then $\operatorname{det} \bar{A} \neq 0$. Using (2.2) it is easy to observe that in this case there exist $t_{1}, \ldots, t_{m} \in \mathfrak{h}$ satisfying $\left[t_{i}, e_{j}\right]=\delta_{i, j} e_{j}$ for all $1 \leq i, j \leq m$. But then $D=\sum_{i=1}^{m} \lambda_{i}$ ad $t_{i} \in$ ad $\mathfrak{g}$, yielding $\operatorname{Der}(\mathfrak{g})=$ ad $\mathfrak{g}$. If $\mathfrak{g}=\mathfrak{p s l}_{m+1}$ with $p \mid(m+1)$, then it is readily seen that there are $t_{1}, \ldots, t_{m} \in \mathfrak{p g l}_{m+1}$ such that $\left[t_{i}, e_{j}\right]=\delta_{i, j} e_{j}$ for all $1 \leq i, j \leq m$. The above discussion then implies that $\operatorname{Der}\left(\mathfrak{p s l}_{m+1}\right) \cong \operatorname{ad} \mathfrak{p g l} \mathfrak{g l}_{m+1} \cong \mathfrak{p g l}_{m+1}$ if $p \mid(m+1)$.

Now suppose that $\mathfrak{g}=\mathfrak{p g l}_{m+1}$ with $p \mid(m+1)$, and let $D$ be any derivation of $\mathfrak{g}$. Clearly, $D$ induces a derivation of $\mathfrak{g}^{(1)}=\mathfrak{p s l}_{m+1}$. By the above, there exists an element $x \in \mathfrak{g}$ such that $D-\operatorname{ad} x$ acts trivially on $\mathfrak{g}^{(1)}$. We now let $y$ be any element of $\mathfrak{g}$. Then

$$
0=(D-\operatorname{ad} x)\left(\left[y, \mathfrak{g}^{(1)}\right]\right)=\left[(D-\operatorname{ad} x)(y), \mathfrak{g}^{(1)}\right],
$$

implying that $(D-\operatorname{ad} x)(y)$ belongs to the centralizer of $\mathfrak{g}^{(1)}$ in $\mathfrak{g}$. Since $p>2$, it is straightforward to see that the latter is trivial. Therefore, $D=\operatorname{ad} x$, completing the proof.

In Section 1.12, we have used certain exponential automorphisms. Next we establish the properties required there.

Lemma 2.8. Suppose $\mathfrak{g}$ is a classical simple Lie algebra, or a Lie algebra isomorphic to $\mathfrak{s l}_{m+1}, \mathfrak{g l}_{m+1}$, or $\mathfrak{p g l}_{m+1}$ where $p \mid(m+1)$. If $x \in \mathfrak{g}^{\alpha}$ is a root vector, then $\exp (\operatorname{ad} x)$ is an automorphism of $\mathfrak{g}$.

Proof. If $x \in \mathfrak{g}^{\alpha}$ is a root vector, then $(\operatorname{ad} x)^{3}=0$ on $\mathfrak{g}$, except when $\mathfrak{g}$ is of type $\mathrm{G}_{2}$ and $\alpha$ is a short root. In this exceptional case, ad $x$ is nilpotent of 
order 4. Thus, except for root vectors corresponding to short roots in type $\mathrm{G}_{2}$ in characteristic 5 , we have $(\operatorname{ad} x)^{\frac{p+1}{2}}=0$ for all root vectors.

Now if $D$ is any derivation of $\mathfrak{g}$ with $D^{\frac{p+1}{2}}=0$, then using the well-known Leibniz formula,

$$
\frac{D^{n}([y, z])}{n !}=\sum_{i=0}^{n}\left[\frac{D^{i}(y)}{i !}, \frac{D^{n-i}(z)}{(n-i) !}\right]
$$

we have

$$
\begin{aligned}
{[\exp (D)(y), \exp (D)(z)] } & =\left[\sum_{i=0}^{\frac{p-1}{2}} \frac{D^{i}(y)}{i !}, \sum_{j=0}^{\frac{p-1}{2}} \frac{D^{j}(z)}{j !}\right] \\
& =\sum_{n=0}^{p-1} \sum_{i=0}^{n}\left[\frac{D^{i}(y)}{i !}, \frac{D^{n-i}(z)}{(n-i) !}\right]=\sum_{n=0}^{p-1} \frac{D^{n}([y, z])}{n !} \\
& =\exp (D)([y, z]),
\end{aligned}
$$

so that $\exp (D)$ is Lie homomorphism of $\mathfrak{g}$. If $D^{\prime}$ is another derivation of $\mathfrak{g}$ with $\left(D^{\prime}\right)^{\frac{p+1}{2}}=0$ and $\left[D, D^{\prime}\right]=0$, then

$$
\begin{aligned}
\exp (D) \exp \left(D^{\prime}\right) & =\left(\sum_{i=0}^{\frac{p-1}{2}} \frac{D^{i}}{i !}\right)\left(\sum_{j=0}^{\frac{p-1}{2}} \frac{\left(D^{\prime}\right)^{j}}{j !}\right) \\
& =\sum_{k=0}^{p-1} \frac{\left(D+D^{\prime}\right)^{k}}{k !}=\exp \left(D+D^{\prime}\right) .
\end{aligned}
$$

Taking $D^{\prime}=-D$ shows that $\exp (D) \exp (-D)=I$, so that $\exp (D)$ is an automorphism of $\mathfrak{g}$. As a result, $\exp (\operatorname{ad} x)$ is an automorphism of $\mathfrak{g}$ for all root vectors, except when $x$ corresponds to a short root in type $\mathrm{G}_{2}$ and characteristic 5 .

In the exceptional case, we suppose that $\mathfrak{g}$ is a Lie algebra of type $\mathrm{G}_{2}$ in characteristic 5 and adopt the labelling of the roots as in [Bou1, Planche IX] so that $\alpha_{1}, \alpha_{2}$ are the simple roots, and the positive roots are $\left\{\alpha_{1}, \alpha_{2}, \alpha_{1}+\right.$ $\left.\alpha_{2}, 2 \alpha_{1}+\alpha_{2}, 3 \alpha_{1}+\alpha_{2}, 3 \alpha_{1}+2 \alpha_{2}\right\}$. Then for $x \in \mathfrak{g}^{\alpha}$ ( $\alpha$ a root) and $y \in \mathfrak{g}^{\beta}$ $(\beta$ a root or 0$)$, we have $(\operatorname{ad} x)^{3} y=0$ unless $\alpha$ is short. By applying a root system isomorphism, we may assume $\alpha=\alpha_{1}, x \in \mathfrak{g}^{\alpha_{1}}$, and $y, z$ are root vectors in $\mathfrak{g}$. Then

$$
\begin{aligned}
{[\exp (\operatorname{ad} x)(y), \exp (\operatorname{ad} x)(z)]=\exp (\operatorname{ad} x)([y, z]) } & \\
+ & {\left[\frac{(\operatorname{ad} x)^{2}(y)}{2 !}, \frac{(\operatorname{ad} x)^{3}(z)}{3 !}\right] } \\
+ & {\left[\frac{(\operatorname{ad} x)^{3}(y)}{3 !}, \frac{(\operatorname{ad} x)^{2}(z)}{2 !}\right] } \\
+ & {\left[\frac{(\operatorname{ad} x)^{3}(y)}{3 !}, \frac{(\operatorname{ad} x)^{3}(z)}{3 !}\right] . }
\end{aligned}
$$


Now $(\operatorname{ad} x)^{3}(\mathfrak{g})$ is spanned by the root vectors corresponding to the roots $3 \alpha_{1}+\alpha_{2}$ and $-\alpha_{2}$, and $(\operatorname{ad} x)^{2}(\mathfrak{g})$ is spanned modulo $(\operatorname{ad} x)^{3}(\mathfrak{g})$ by root vectors corresponding to $2 \alpha_{1}+\alpha_{2}, \alpha_{1}$, and $-\alpha_{1}-\alpha_{2}$. From this it is easy to see that $(\operatorname{ad} x)^{3}(\mathfrak{g})$ is central in $(\operatorname{ad} x)^{2}(\mathfrak{g})$, so that $[\exp (\operatorname{ad} x)(y), \exp (\operatorname{ad} x)(z)]$ $=\exp (\operatorname{ad} x)([y, z])$ and $\exp (\operatorname{ad} x)$ is a homomorphism. Because

$$
\begin{aligned}
\exp (\operatorname{ad} x) \exp (-\operatorname{ad} x) & =\left(\sum_{i=0}^{p-1} \frac{(\operatorname{ad} x)^{i}}{i !}\right)\left(\sum_{j=0}^{p-1} \frac{(-\operatorname{ad} x)^{j}}{j !}\right) \\
& =\sum_{n=0}^{p-1} \sum_{k=0}^{n} \frac{(\operatorname{ad} x)^{n-k}}{(n-k) !} \frac{(-\operatorname{ad} x)^{k}}{k !} \\
& +\sum_{i+j=p}^{2 p-2}(-1)^{j} \frac{(\operatorname{ad} x)^{i+j}}{i ! j !} \\
& =\sum_{n=0}^{p-1} \frac{(\operatorname{ad} x-\operatorname{ad} x)^{n}}{n !}=I,
\end{aligned}
$$

the mapping $\exp (\operatorname{ad} x)$ is an automorphism as claimed.

Each classical Lie algebra possesses a finite reduced root system $\Phi$. Corresponding to such a root system $\Phi$ is the associated Weyl group $W$, which is generated by the reflections $s_{\alpha} \in \operatorname{End}\left(\mathfrak{h}^{*}\right), \alpha \in \Phi$,

$$
s_{\alpha}(\mu)=\mu-\langle\mu, \alpha\rangle \alpha \text {. }
$$

In fact, it can be shown that $W$ is generated by the reflections $s_{\alpha_{i}}$, $1 \leq i \leq m$, in hyperplanes perpendicular to the simple roots.

Suppose $P=\mathbb{Z} \varpi_{1} \oplus \cdots \oplus \mathbb{Z} \varpi_{m}$ is the weight lattice corresponding to $\Phi$, and let $P^{+}=\mathbb{N} \varpi_{1} \oplus \cdots \oplus \mathbb{N} \varpi_{m}\left(\mathbb{N}=\mathbb{Z}_{\geq 0}\right)$ be the set of dominant (integral) weights. A subset $X$ of $P$ is said to be saturated if for all $\lambda \in X$ and $\alpha \in \Phi$, the weight $\lambda-t \alpha \in X$ for all integers $t$ between 0 and $\langle\lambda, \alpha\rangle$, inclusive. (See [Bou2, Chap. VIII, Sec. 7, No. 2], or [H3, Sec. 13.4].)

The minuscule weights $\lambda \in P^{+}$will play a role in our subsequent deliberations. They have several equivalent characterizations:

(i) The Weyl group orbit $W \lambda$ of $\lambda$ is the smallest saturated set of weights in $P$ containing $\lambda$.

(ii) For all roots $\alpha \in \Phi,\langle\lambda, \alpha\rangle \in\{-1,0,1\}$.

(iii) If $\mu \in P^{+}$and $\mu<\lambda$, (i.e., $\lambda-\mu=\sum_{i=1}^{m} k_{i} \alpha_{i}$ where $k_{i} \in \mathbb{N}$ for all $i)$, then $\lambda=\mu$.

Relatively few weights are minuscule, and they are displayed below: 


$\begin{aligned} \mathrm{A}_{m} & \varpi_{1}, \ldots, \varpi_{m} \\ \mathrm{~B}_{m} & \varpi_{m} \\ \mathrm{C}_{m} & \varpi_{1} \\ \mathrm{D}_{m} & \varpi_{1}, \varpi_{m-1}, \varpi_{m} \\ \mathrm{E}_{6} & \varpi_{1}, \varpi_{6} \\ \mathrm{E}_{7} & \varpi_{7} .\end{aligned}$

The Borel subalgebras

$$
\begin{aligned}
\mathfrak{b}^{+} & :=\mathfrak{h} \oplus \bigoplus_{\alpha>0} \mathfrak{g}^{\alpha} \\
\mathfrak{b}^{-} & :=\mathfrak{h} \oplus \bigoplus_{\alpha>0} \mathfrak{g}^{-\alpha}
\end{aligned}
$$

are essential in the structure and representation theory of $\mathfrak{g}$. Unlike the situation in characteristic zero where Lie's Theorem applies, a finite-dimensional $\mathfrak{g}$-module $M$ need not possess a common eigenvector for $\mathfrak{b}^{+}$(or for $\mathfrak{b}^{-}$). However, when such a common eigenvector $v$ exists for $\mathfrak{b}^{+}$(resp. for $\mathfrak{b}^{-}$), it must be annihilated by $\mathfrak{n}^{+}:=\bigoplus_{\alpha>0} \mathfrak{g}^{\alpha}$ (resp. $\mathfrak{n}^{-}:=\bigoplus_{\alpha>0} \mathfrak{g}^{-\alpha}$ ) and be a weight vector for $\mathfrak{h}$. This motivates the following definition.

DeFinition 2.10. Let $M$ be a finite-dimensional module for the classical algebra $\mathfrak{g}$. A nonzero vector $v$ in $M$ is said to be a $\mathfrak{b}^{+}$-primitive (resp. $\mathfrak{b}^{-}$primitive) vector of weight $\lambda$ if $x . v=0$ for all $x \in \mathfrak{n}^{+}$(resp. $\left.x \in \mathfrak{n}^{-}\right)$and $h . v=\lambda(h) v$ for all $h \in \mathfrak{h}$.

As explained in Remark 1.70, any finite-dimensional irreducible $\mathfrak{g}$-module which is restricted over $\mathfrak{g}^{(1)}$ possesses $\mathfrak{a} \mathfrak{b}^{+}$-primitive vector (and by symmetry, a $\mathfrak{b}^{-}$-primitive vector).

\subsection{Representations of algebraic groups}

The automorphism group of a classical simple Lie algebra is an algebraic group of adjoint type. Various proofs in the text will require us to work with the simply connected cover of such a group. In this section, we assemble the information we will need about algebraic groups.

Let $G$ be a linear algebraic group, that is, a Zariski closed subgroup of $\operatorname{GL}(V)$ where $V$ is a finite-dimensional vector space over $\mathbb{F}$. The group $G$ is said to be connected if $G$ equals the connected component $G^{\circ}$ of $G$ (the unique irreducible component of $G$ containing the identity element). Let 
$A=\mathbb{F}[G]$ denote the coordinate ring of $G$. Then $G$ acts on $A$ via left (resp. right) translation: $\left(\lambda_{x} f\right)(y)=f\left(x^{-1} y\right)$ (resp. $\left(\rho_{x} f\right)(y)=f(y x)$ ). The space $\operatorname{Lie}(G):=\left\{d \in \operatorname{Der}(A) \mid d \lambda_{x}=\lambda_{x} d\right.$ for all $\left.x \in G\right\}$ of all leftinvariant derivations of the ring $A$ is a Lie algebra, since the bracket of two derivations which commute with $\lambda_{x}$ obviously does likewise. We call $\operatorname{Lie}(G)$ the Lie algebra of $G$. Since the $p$-th power of any left-invariant derivation of $A$ is again a left-invariant derivation, the Lie algebra $\operatorname{Lie}(G)$ carries a canonical restricted Lie algebra structure.

We now gather a few basic facts from Section 31 of [H2]. Let $G$ be a semisimple algebraic group. Assume $T$ is a maximal torus of $G, B^{+}=T N^{+}$ is a Borel subgroup containing $T$, and $B^{-}=T N^{-}$is the opposite Borel subgroup. Suppose $\Delta$ is the base of the root system $\Phi$ determined by $B^{+}$. Thus, $\Phi$ is the set of nonzero weights of $\operatorname{Ad} T$ in $\operatorname{Lie}(G)$. The Weyl group $W$ of $G$ is defined to be $N_{G}(T) / Z_{G}(T)$, the normalizer modulo the centralizer of the torus $T$.

Let $X(T)$ denote the lattice of rational characters of the torus $T$. The elements of $X(T)$ are often referred to as weights. Relative to the base $\Delta=$ $\left\{\alpha_{1}, \ldots, \alpha_{m}\right\}$, the fundamental weights $\left\{\varpi_{1}, \ldots, \varpi_{m}\right\} \subset X(T) \otimes_{\mathbb{Z}} \mathbb{Q}$ satisfy $\left(\varpi_{i}, \alpha_{j}^{\vee}\right)=\delta_{i, j}$, where $\beta^{\vee}=2(\beta, \beta)^{-1} \beta$ for any $\operatorname{root} \beta$. If $\varpi_{i} \in X(T)$ for all $i$, then $G$ is said to be simply connected. In this case, the fundamental weights form a basis of the $\mathbb{Z}$-module $X(T)$. (By identifying weights $\nu$ with their differentials $(\mathrm{d} \nu)_{e}$, we are able to use the same notation for the fundamental weights in $X(T)$ as for the fundamental weights of the corresponding Lie algebra.)

A rational representation of $G$ is a homomorphism of algebraic groups $\varrho: G \rightarrow \mathrm{GL}(M)$ for some finite-dimensional vector space $M$. We say the resulting $G$-module $M$ is rational. Every rational $G$-module $M$ admits a weight space decomposition

$$
M=\bigoplus_{\lambda \in X(T)} M^{\lambda}
$$

where $M^{\lambda}=\left\{v \in M \mid t v=\chi_{\lambda}(t) v\right.$ for all $\left.t \in T\right\}$ (here $\chi_{\lambda}$ is the rational character of $T$ corresponding to $\lambda \in X(T)$ ). Whenever $M^{\lambda} \neq 0$, then $\lambda$ is said to be a weight of $M$ with respect to $T$. Using the fact that the Weyl group $W=N_{G}(T) / Z_{G}(T)$, it is routine to check that $W$ permutes the weights of $M$, and $\operatorname{dim} M^{\lambda}=\operatorname{dim} M^{w \lambda}$ for all $w \in W$. We let $X(M)$ denote the set of weights of the module $M$.

From now on we suppose that $G$ is simply connected. Set 


$$
\begin{aligned}
& X(T)_{+}:=\bigoplus_{i=1}^{m} \mathbb{N} \varpi_{i}, \quad\left(\mathbb{N}=\mathbb{Z}_{\geq 0}\right) \\
& X_{1}(T):=\left\{\sum_{i=1}^{m} a_{i} \varpi_{i} \mid a_{i} \in \mathbb{N}, 0 \leq a_{i} \leq p-1\right\} \\
& Q_{+}:=\bigoplus_{i=1}^{m} \mathbb{N} \alpha_{i}
\end{aligned}
$$

Elements of $X(T)_{+}$are called the dominant weights of $T$ (with respect to the base $\Delta)$. There is a partial ordering $\leq$ on $X(T)$ defined by

$$
\mu \leq \lambda \Longleftrightarrow \lambda-\mu \in Q_{+}
$$

Let $X(M)$ denote the set of weights of the module $M$. Then $\lambda \in X(M)$ is a maximal (resp. minimal) weight of $M$ if $\mu \geq \lambda$ (resp. $\mu \leq \lambda$ ) for $\mu \in X(M)$ implies $\mu=\lambda$. If $\lambda$ is maximal weight of $M$, then $\lambda \in X(T)_{+}$, and if $\lambda$ is a minimal weight, then $\lambda \in-X(T)_{+}$. An irreducible $G$-module $M$ has a unique maximal and a unique minimal weight, and the corresponding weight spaces are one-dimensional. Any two finite-dimensional irreducible rational $G$-modules are isomorphic if and only if they have the same maximal (minimal) weight.

Any $\lambda \in X(T)$ may be extended to all of $B^{+}$by setting $\lambda(u)=1$ for all $u \in N^{+}$. Then $\lambda$ determines a one-dimensional $B^{+}$-module $\mathbb{F}_{\lambda}$, where the $B^{+}$-action is afforded by $\lambda$. Let $H^{0}(\lambda)=\operatorname{ind}_{B^{+}}^{G} \mathbb{F}_{\lambda} \cong\{f \in \mathbb{F}[G]$ $f(g b)=\lambda(b)^{-1} f(g)$ for all $g \in G$ and $\left.b \in B^{+}\right\}$be the corresponding induced module. The socle $L(\lambda):=\operatorname{soc}_{G} H^{0}(\lambda)$ is a simple $G$-module. Thus, for any $\lambda \in X(T)_{+}$there exists a finite-dimensional irreducible rational $G$ module $L(\lambda)$ with maximal weight $\lambda$. Although the dimension of $L(\lambda)$ is very difficult to determine, in general, one knows that $\operatorname{dim} H^{0}(\lambda)$ is given by Weyl's dimension formula:

$$
\operatorname{dim} H^{0}(\lambda)=\prod_{\alpha \in \Phi^{+}} \frac{(\lambda+\rho, \alpha)}{(\rho, \alpha)},
$$

where $\rho$ is half the sum of the positive roots (this is immediate from $[\mathbf{J a}$, Part II, Prop. 5.10]).

Proposition 2.12. (Compare [Ja, Part II, Cor. 2.5].) The module dual to $L(\lambda)$ is $L\left(-w_{0} \lambda\right)$, where $w_{0}$ is the longest element of the Weyl group $W$.

For any rational representation $\varrho: G \rightarrow \mathrm{GL}(V)$, the differential map

$$
(\mathrm{d} \varrho)_{e}: \operatorname{Lie}(G) \rightarrow \mathfrak{g l}(V)
$$


is a homomorphism of restricted Lie algebras. Thus, any rational $G$-module $L(\lambda)$ can be regarded, canonically, as a restricted $\operatorname{Lie}(G)$-module. The following classical result is due to Curtis $[\mathbf{C}]$ :

Proposition 2.13. (Compare [Ja, Part II, Prop. 3.10 and Prop. 3.15].) Any irreducible restricted $\operatorname{Lie}(G)$-module is isomorphic to exactly one module $L(\lambda)$ with $\lambda \in X_{1}(T)$. Conversely, any $G$-module $L(\lambda)$ with $\lambda \in X_{1}(T)$ is irreducible as a $\operatorname{Lie}(G)$-module.

The module $V(\lambda):=H^{0}\left(-w_{0} \lambda\right)^{*}$ which is dual to $H^{0}\left(-w_{0} \lambda\right)$ is called the Weyl module corresponding to $\lambda$. There is an antiautomorphism $\tau$ of order 2 on $G$ which acts as the identity on $T$ and sends the root subgroup $U_{\alpha}$ to $U_{-\alpha}$, for each $\alpha \in \Phi$ (as in [Ja, Part II, Sec. 1.2]). Then $V(\lambda) \cong{ }^{\tau} H^{0}(\lambda)$, where the $G$-action on the right is twisted by $\tau$. Using that fact, one can show that the Weyl module $V(\lambda)$ is generated by an eigenvector $v$ for $B^{+}$ corresponding to $\lambda$. Moreover, we have the following:

Proposition 2.14. (See [Ja, Part II, Sec. 2.13, 2.14(1)].)

(a) Any rational $G$-module $M$ which is generated by a $B^{+}$-eigenvector $m$ of weight $\lambda$ is a homomorphic image of $V(\lambda)$ via a homomorphism sending $v$ to $m$.

(b) If $\operatorname{rad}_{G} V(\lambda)$ denotes the intersection of all maximal submodules of $V(\lambda)$, then $V(\lambda) / \operatorname{rad}_{G} V(\lambda) \cong L(\lambda)$.

The result stated below will play a crucial role in Chapter 4 of this monograph (recall that we are assuming $G$ is simply connected):

Proposition 2.15. ([Ja, Part II, Prop. 9.24(b)]; see also [Ja, Part I, Sec. 9.6].) Let $M$ be a finite-dimensional rational $G$-module such that

$$
X_{+}(M):=\left(X(M) \cap X(T)_{+}\right) \subseteq X_{1}(T) .
$$

Then any subspace of $M$ invariant under the induced action of $\operatorname{Lie}(G)$ is a $G$-submodule of $M$.

\subsection{Standard gradings of classical Lie algebras}

In this section, we assume that $\mathfrak{g}$ is either a classical simple Lie algebra or $\mathfrak{p g l}_{m+1}$ with $p \mid m+1$. Our goal here is to recall the notion of a standard grading of $\mathfrak{g}$ and to describe the gradings $\mathfrak{g}=\bigoplus_{j \in \mathbb{Z}} \mathfrak{g}_{j}$ of $\mathfrak{g}$ such that $\mathfrak{g}_{-1}$ is an irreducible $\mathfrak{g}_{0}$-module and $\mathfrak{g}_{-}$is generated by $\mathfrak{g}_{-1}$.

As explained in Section 2.2, $\mathfrak{g}=\mathfrak{t} \oplus \bigoplus_{\alpha \in \Phi} \mathfrak{g}^{\alpha}$, where $\mathfrak{t}$ is an abelian Cartan subalgebra of $\mathfrak{g}$, and $\left[\mathfrak{t}, \mathfrak{g}^{\alpha}\right]=\mathfrak{g}^{\alpha}$ for all $\alpha \in \Phi$. Moreover, each

root space $\mathfrak{g}^{\alpha}$ is one-dimensional, and $\exp (\operatorname{ad} x) \in$ Aut $\mathfrak{g}$ for all root vectors 
$x \in \bigcup_{\alpha \in \Phi} \mathfrak{g}^{\alpha}$; see Lemma 2.8. As in Section 2.2, we regard $\Phi$ as a root system in an Euclidean space. Let $\Delta=\left\{\alpha_{1}, \ldots, \alpha_{m}\right\}$ be a basis of simple roots in $\Phi$. Recall that every root in $\Phi$ is a nonnegative or nonpositive integral linear combination of roots in $\Delta$.

Associated to any $m$-tuple of nonnegative integers $\left(a_{1}, \ldots, a_{m}\right)$, there is a grading of the Lie algebra $\mathfrak{g}$ in which a root vector corresponding to a root $\alpha=\sum_{i=1}^{m} n_{i} \alpha_{i}$ is given gradation degree $\sum_{i=1}^{m} a_{i} n_{i}$ and all elements in $\mathfrak{t}$ are assigned gradation degree 0 . If $\sum_{i=1}^{m} a_{i}=1$, i.e. if $a_{k}=1$ for some $k \in\{1, \ldots, m\}$ and $a_{j}=0$ for $j \neq k$, then we say that the grading is standard.

Two gradings $L=\bigoplus_{j \in \mathbb{Z}} L_{j}$ and $L=\bigoplus_{j \in \mathbb{Z}} L_{j}^{\prime}$ of a Lie algebra $L$ are said to be conjugate if there is an automorphism $\sigma$ of $L$ such that $\sigma\left(L_{j}\right)=L_{j}^{\prime}$ for all $j \in \mathbb{Z}$. In this monograph, we identify conjugate gradings without further notice. Our next result is known to the experts; we provide a short proof for the reader's convenience.

Proposition 2.16. Every grading $\mathfrak{g}=\bigoplus_{j \in \mathbb{Z}} \mathfrak{g}_{j}$ of the Lie algebra $\mathfrak{g}$ such that $\mathfrak{g}_{-1}$ is an irreducible $\mathfrak{g}_{0}$-module and $\mathfrak{g}_{-}$is generated by $\mathfrak{g}_{-1}$ is standard.

Proof. Let $G:=(\text { Aut } \mathfrak{g})^{\circ}$, the connected component of the identity in the automorphism group of $\mathfrak{g}$, which is a simple algebraic $\mathbb{F}$-group of adjoint type. It is well-known that $\operatorname{Lie}(G)$ is isomorphic to a Lie subalgebra of $\operatorname{Der}(\mathfrak{g})$. In view of Lemma 2.8, we have that ad $\mathfrak{g}^{\alpha} \subset \operatorname{Lie}(G)$ for all $\alpha \in \Phi$. If $\mathfrak{g}$ is not of type $\mathrm{A}_{m}$ with $p \mid(m+1)$, then $\mathfrak{g}$ is a simple Lie algebra and $\operatorname{Der}(\mathfrak{g})=$ ad $\mathfrak{g}$. Since $\bigcup_{\alpha \in \Phi}$ ad $\mathfrak{g}^{\alpha}$ generates a nonzero ideal of ad $\mathfrak{g}$, it follows that $\operatorname{Lie}(G)=\operatorname{ad} \mathfrak{g}$ in this case. If $\mathfrak{g}$ is of type $\mathrm{A}_{m}$ with $p \mid(m+1)$, then either $\mathfrak{g}=\mathfrak{p s l}_{m+1}$ or $\mathfrak{g}=\mathfrak{p g l}_{m+1}$ by our assumption. In this case, $\operatorname{Der}(\mathfrak{g}) \cong \mathfrak{p g l}_{m+1}$ implying $\operatorname{Lie}(G) \cong \mathfrak{p g l}_{m+1}$. Thus in any event, ad $\mathfrak{g}$ is an ideal of codimension $\leq 1$ in $\operatorname{Lie}(G)$.

For ease of notation, we identify $\mathfrak{g}$ with ad $\mathfrak{g} \subseteq \operatorname{Lie}(G)$. It follows from the preceding remark that $G$ contains a maximal torus $T$ such that $\operatorname{Lie}(T) \cap$ $\mathfrak{g}=\mathfrak{t}$. Moreover, the theory of algebraic groups enables us to identify $\Phi$ with the root system of $G$ relative to $T$.

Given a linear algebraic $\mathbb{F}$-group $H$, we denote by $X_{*}(H)$ the set of all rational homomorphisms from the one-dimensional torus $\mathbb{F}^{\times}$to $H$. Associated with our grading $\mathfrak{g}=\bigoplus_{j \in \mathbb{Z}} \mathfrak{g}_{j}$ is a rational homomorphism $\lambda \in X_{*}(G)$ such that

$$
\left.\lambda(t)\right|_{\mathfrak{g}_{j}}=t^{j} \operatorname{id}_{\mathfrak{g}_{j}} \quad\left(\forall t \in \mathbb{F}^{\times}, \forall j \in \mathbb{Z}\right) .
$$

Clearly, $\lambda\left(\mathbb{F}^{\times}\right)$is a one-dimensional torus of $G$. Since any torus of $G$ is contained in a maximal torus and all maximal tori of $G$ are conjugate, we can assume in the rest of the proof that $\lambda\left(\mathbb{F}^{\times}\right) \subseteq T$. In other words, we can assume that $\lambda$ belongs to $X_{*}(T)$, the lattice of rational cocharacters of $T$. Note that the Weyl group $W=N_{G}(T) / T$ acts on $X_{*}(T)$. 
Let $X(T)$ be the lattice of characters of $T$; see Section 2.3. According to $[$ Bo, $(8.6)]$, the $\mathbb{Z}$-pairing

$$
X(T) \times X_{*}(T) \rightarrow \mathbb{Z}, \quad(\chi, \nu) \mapsto n:=\langle\chi, \nu\rangle \quad \text { if } \quad \chi(\nu(t))=t^{n},
$$

is $W$-invariant and nondegenerate. As $G$ is a group of adjoint type, the lattice $X(T)$ is spanned over $\mathbb{Z}$ by the set of simple roots $\Delta$. Consequently, the pairing (2.18) identifies $X_{*}(T)$ with $P$, the weight lattice corresponding to $\Phi$. Replacing $\lambda$ by its $W$-conjugate if necessary, we can assume in what follows that $\lambda$ lies in the dual Weyl chamber corresponding to $\Delta$. That is to say, we can assume that $\alpha_{i}(\lambda(t))=t^{a_{i}}$ for some nonnegative integers $a_{i}$, where $1 \leq i \leq m$.

In conjunction with (2.17), the above discussion shows that $\mathfrak{g}_{0}$ is spanned by $\mathfrak{t}$ and the root spaces $\mathfrak{g}^{\alpha}$ with $\alpha=\sum_{i=1}^{m} n_{i} \alpha_{i}$ satisfying $n_{k}=0$ whenever $a_{k} \neq 0$. It also shows that $\mathfrak{g}_{-1}$ is spanned by the root spaces $\mathfrak{g}^{\gamma}$ with $\gamma=\sum_{i=1}^{m} r_{i} \alpha_{i}$ for which $\sum_{i=1}^{m} r_{i} a_{i}=-1$. All such $\gamma^{\prime}$ 's are negative roots in $\Phi$. Since $\mathfrak{g}_{-1} \neq 0$, it must be that $a_{d}=1$ for some $d \in\{1, \ldots, m\}$.

Let $\mathfrak{g}_{-1, d}$ denote the span of all $\mathfrak{g}^{\gamma} \subset \mathfrak{g}_{-1}$ with $\gamma=\sum_{i=1}^{m} r_{i} \alpha_{i}$ such that $r_{d}=-1$. Since $\mathfrak{g}^{-\alpha_{d}} \subseteq \mathfrak{g}_{-1, d}$, we have $\mathfrak{g}_{-1, d} \neq 0$. Moreover, because $\mathfrak{g}_{-1}$ is an irreducible $\mathfrak{g}_{0}$-module, our description of $\mathfrak{g}_{0}$ now implies that $\mathfrak{g}_{-1}=\mathfrak{g}_{-1, d}$. Suppose $a_{j} \neq 0$ for some $j \neq d$. Then $\mathfrak{g}^{-\alpha_{j}} \subseteq \mathfrak{g}_{-}$. On the other hand, if $\mathfrak{g}^{-\beta}$ lies in the Lie subalgebra generated by $\mathfrak{g}_{-1, d}$, then $\beta=\sum_{i=1}^{m} q_{i} \alpha_{i}$ with $q_{d} \geq 1$. It follows that $\mathfrak{g}^{-\alpha_{j}}$ is not contained in the subalgebra generated by $\mathfrak{g}_{-1, d}=\mathfrak{g}_{-1}$. Since this contradicts one of our assumptions, we derive that $a_{j}=0$ for $j \neq d$. Thus, our grading is standard, as stated.

\subsection{The Lie algebras of Cartan type}

There are four infinite series of finite-dimensional simple, graded Cartan type Lie algebras $\mathfrak{g}=\bigoplus_{j=-q}^{r} \mathfrak{g}_{j}$ : the Witt, special, Hamiltonian, and contact series. The Lie algebras in each series vary in "thickness" (i.e., dimensions of corresponding gradation spaces $\mathfrak{g}_{j}$ ) and "length" (i.e., number of nonzero gradation spaces). The special, Hamiltonian, and contact Lie algebras are subalgebras of the Witt Lie algebras. The Witt, special, and Hamiltonian Lie algebras all have depth-one gradations $(q=1)$ with respect to which they are transitive (1.2) and irreducible (1.1); while the contact Lie algebras have a depth-two gradation $(q=2)$, which is transitive and irreducible.

Each series contains both restricted and nonrestricted Lie algebras. Each nonrestricted algebra contains a restricted subalgebra (its "initial piece"). The restricted ones, which are exactly the initial pieces of the algebras in a given series, are generated as Lie algebras by their local part $\mathfrak{g}_{-1} \oplus \mathfrak{g}_{0} \oplus \mathfrak{g}_{1}$. The local part of a nonrestricted graded Cartan type Lie algebra coincides with the local part of its initial piece. 
When $\mathfrak{g}$ is a restricted Cartan type Lie algebra, then $x^{[p]}=0$ for $x \in \mathfrak{g}_{-1}$, since $(\operatorname{ad} x)^{p}$ is a derivation which annihilates the local part when $p>3$. The restriction of the $[p]$-mapping of $\mathfrak{g}$ to $\mathfrak{g}_{0}$ coincides with the $[p]$-mapping on the classical Lie algebra $\mathfrak{g}_{0}^{(1)}$, and the adjoint representation of $\mathfrak{g}_{0}$ on each $\mathfrak{g}_{j}$ is a $p$-representation, i.e., is restricted.

We will illustrate all these concepts by beginning with a few simple examples of Cartan type Lie algebras. Then we will introduce the divided power algebras and their derivations and use them to describe the four infinite series of Cartan type Lie algebras.

\subsection{The Jacobson-Witt algebras}

The Jacobson-Witt Lie algebras were the first finite-dimensional nonclassical simple Lie algebras to be discovered. Their construction starts with a polynomial algebra $\mathbb{F}\left[x_{1}, \ldots, x_{m}\right]$ over $\mathbb{F}$, a field of characteristic $p>0$. Let $D_{i}=\partial / \partial x_{i}, i=1, \ldots, m$, be the usual partial derivatives relative to the variables $x_{i}$. The ideal generated by the elements $x_{1}^{p}, \ldots, x_{m}^{p}$ is invariant under the derivations $D_{i}$, and so the $D_{i}$ induce derivations on the truncated polynomial algebra $\mathbb{F}\left[x_{1}, \ldots, x_{m}\right] /\left\langle x_{1}^{p}, \ldots, x_{m}^{p}\right\rangle$. The Jacobson-Witt Lie algebra is the derivation algebra $W(m ; \underline{1}):=\operatorname{Der}\left(\mathbb{F}\left[x_{1}, \ldots, x_{m}\right] /\left\langle x_{1}^{p}, \ldots, x_{m}^{p}\right\rangle\right)$.

In the special case that $m=1$, the resulting Lie algebra $W(1 ; \underline{1})$ is called the $p$-dimensional Witt algebra or simply the Witt algebra. The JacobsonWitt algebras may be viewed as "thickenings" of it by addition of variables.

By identifying cosets with their representatives, we may assume that the elements $x^{a}:=x_{1}^{a_{1}} \cdots x_{m}^{a_{m}}$ with $a=\left(a_{1}, \ldots, a_{m}\right)$ and $0 \leq a_{i}<p$ for all $i$ determine a basis for $\mathbb{F}\left[x_{1}, \ldots, x_{m}\right] /\left\langle x_{1}^{p}, \ldots, x_{m}^{p}\right\rangle$. Then the derivations $x^{a} D_{i}$, as $a$ ranges over such $m$-tuples and $i=1, \ldots, m$, comprise a basis for $W(m ; \underline{1})$. The Lie bracket in $W(m ; \underline{1})$ is given by

$$
\left[x^{a} D_{i}, x^{b} D_{j}\right]=b_{i} x^{a+b-\epsilon_{i}} D_{j}-a_{j} x^{a+b-\epsilon_{j}} D_{i},
$$

where $\epsilon_{i}$ is the $m$-tuple with 1 in the $i$ th position and 0 elsewhere, and addition of tuples is componentwise. If any component exceeds $p-1$ or is less than 0 , the term is 0 .

Because the Lie algebra $W(m ; \underline{1})$ is the derivation algebra of an algebra, it carries a natural $[p]$-structure given by the $p$-mapping:

$$
D^{[p]}=D^{p} \quad \text { for all } D \in W(m ; \underline{1}) .
$$

Rather than working with monomials as above, we could use divided powers instead. Thus, if we adopt the basis $x^{(a)}=\frac{x_{1}^{a_{1}} \cdots x_{m}^{a_{m}}}{a_{1} ! \cdots a_{m} !}$, where again $0 \leq a_{i}<p$ for all $i$, then 


$$
x^{(a)} x^{(b)}=\left(\begin{array}{c}
a+b \\
a
\end{array}\right) x^{(a+b)}, \quad \text { where } \quad\left(\begin{array}{c}
a+b \\
a
\end{array}\right)=\prod_{j=1}^{m}\left(\begin{array}{c}
a_{j}+b_{j} \\
a_{j}
\end{array}\right) .
$$

Moreover, $W(m ; \underline{1})$ has a basis $\left\{x^{(a)} D_{i} \mid 0 \leq a_{i}<p, i=1, \ldots, m\right\}$ where

$$
\begin{aligned}
D_{i}\left(x^{(a)}\right) & =x^{\left(a-\epsilon_{i}\right)} \quad \text { and } \\
{\left[x^{(a)} D_{i}, x^{(b)} D_{j}\right] } & =\left(\begin{array}{c}
a+b-\epsilon_{i} \\
a
\end{array}\right) x^{\left(a+b-\varepsilon_{i}\right)} D_{j}-\left(\begin{array}{c}
a+b-\epsilon_{j} \\
b
\end{array}\right) x^{\left(a+b-\varepsilon_{j}\right)} D_{i} .
\end{aligned}
$$

It is this form of the algebra that lends itself naturally to further generalizations.

\subsection{Divided power algebras}

Let $\mathbb{F}$ be a field of characteristic $p>0$, and let $\mathcal{O}(m)$ denote the commutative associative algebra with 1 over $\mathbb{F}$ defined by generators $x_{i}^{(k)}$, $1 \leq i \leq m, \quad k \in \mathbb{N}=\mathbb{Z}_{\geq 0}$, which satisfy the relations

$$
\begin{aligned}
x_{i}^{(0)} & =1 \\
x_{i}^{(k)} x_{i}^{(\ell)} & =\left(\begin{array}{c}
k+\ell \\
k
\end{array}\right) x_{i}^{(k+\ell)}, \quad 1 \leq i \leq m, \quad k, \ell \in \mathbb{N} .
\end{aligned}
$$

The "monomials" $x^{(a)}:=x_{1}^{\left(a_{1}\right)} \cdots x_{m}^{\left(a_{m}\right)}, \quad a_{i} \in \mathbb{N}$, constitute a basis of $\mathcal{O}(m)$.

To compute the binomial coefficients in (2.19), it is helpful to consider base $p$-expansions. Assume that the base $p$ expansions of $c$ and $d$ are given by

$$
\begin{aligned}
c & =c_{k} p^{k}+c_{k-1} p^{k-1}+\cdots+c_{1} p+c_{0} \\
d & =d_{k} p^{k}+d_{k-1} p^{k-1}+\cdots+d_{1} p+d_{0}
\end{aligned}
$$

where $0 \leq c_{i}, d_{i}<p$ for all $i$. Then

$$
\left(\begin{array}{l}
c \\
d
\end{array}\right)=\prod_{i=0}^{k}\left(\begin{array}{l}
c_{i} \\
d_{i}
\end{array}\right)
$$

Lemma 2.22. Suppose $c, d \in \mathbb{N}$ satisfy $0 \leq c, d<p^{k+1}$, but $c+d \geq p^{k+1}$. Then $\left(\begin{array}{c}c+d \\ d\end{array}\right)=0$. 
Proof. This will follow from analyzing the "carries" in (2.21) (with top number $c+d$ instead of $c$ ). Let the base $p$ expansions of $c$ and $d$ be as in (2.20). Since $c+d \geq p^{k+1}$, there is some smallest integer $i \geq 0$ so that $c_{i}+d_{i} \geq p$. Then the binomial coefficient $\left(\begin{array}{c}c+d \\ d\end{array}\right)$ must equal 0 , because it contains the factor $\left(\begin{array}{c}c_{i}+d_{i}-p \\ d_{i}\end{array}\right)$, which is 0 .

As a result of Lemma 2.22 , we see for any $m$-tuple $\underline{n}=\left(n_{1}, \ldots, n_{m}\right)$ of positive integers that

$$
\mathcal{O}(m ; \underline{n}):=\operatorname{span}_{\mathbb{F}}\left\{x^{(a)} \mid 0 \leq a_{i}<p^{n_{i}}\right\}
$$

is a subalgebra of the divided power algebra $\mathcal{O}(m)$, and we have containment $\mathcal{O}(m ; \underline{n}) \subseteq \mathcal{O}\left(m ; \underline{n}^{\prime}\right)$ whenever $n_{i} \leq n_{i}^{\prime}$ for all $i=1, \ldots, m$.

\subsection{Witt Lie algebras of Cartan type (the $W$ series)}

Nonrestricted Cartan type Lie algebras can be obtained by "lengthening" the restricted ones; that is, by allowing powers greater than $p-1$ in the divided power variables.

Let $D_{i}(1 \leq i \leq m)$ be the derivation of $\mathcal{O}(m)$ defined by

$$
D_{i}\left(x_{j}^{(r)}\right)=\delta_{i, j} x_{i}^{(r-1)} .
$$

The (infinite-dimensional) Witt Lie algebra of Cartan type is the subalgebra of $\operatorname{Der}(\mathcal{O}(m))$ defined by

$$
W(m):=\operatorname{span}_{\mathbb{F}}\left\{x^{(a)} D_{i} \mid a_{i} \in \mathbb{N}, i=1, \ldots, m\right\},
$$

and having Lie bracket

$$
\left[x^{(a)} D_{i}, x^{(b)} D_{j}\right]=\left(\begin{array}{c}
a+b-\epsilon_{i} \\
a
\end{array}\right) x^{\left(a+b-\epsilon_{i}\right)} D_{j}-\left(\begin{array}{c}
a+b-\epsilon_{j} \\
b
\end{array}\right) x^{\left(a+b-\epsilon_{j}\right)} D_{i} .
$$

It is a free $\mathcal{O}(m)$-module with basis $D_{1}, \ldots, D_{m}$.

The fact that $\mathcal{O}(m ; \underline{n})$ is a subalgebra of $\mathcal{O}(m)$ for any $m$-tuple $\underline{n}$ of positive integers implies that

$$
W(m ; \underline{n}):=\operatorname{span}_{\mathbb{F}}\left\{x^{(a)} D_{i} \mid 0 \leq a_{i}<p^{n_{i}}, i=1, \ldots, m\right\},
$$

is a Lie subalgebra of $W(m)$. We refer to the Lie algebras $W(m ; \underline{n})$ as the Witt Lie algebras of Cartan type, or more succinctly, the Witt algebras.

The simplest examples of Lie algebras of this kind are the $p^{n}$-dimensional Zassenhaus Lie algebras $W(1 ; \underline{n})$ obtained from the divided power algebras 
$\mathcal{O}(1 ; \underline{n})$ for $n=1,2, \ldots$ Each Zassenhaus Lie algebra contains a copy of the $p$-dimensional Witt algebra $W(1 ; \underline{1})$.

More generally, an algebra $W(m ; \underline{n})$ contains a copy of the restricted Lie algebra $W(m ; \underline{1})$ by taking the derivations $\sum_{i=1}^{m} f_{i} D_{i}$ with $f_{i} \in \mathcal{O}(m ; \underline{1})$.

Theorem 2.24. (See for example, [SF, Prop. 2.2 and Thm. 2.4].)

(i) $W(m ; \underline{n})$ is a simple Lie algebra except when $m=1$ and $p=2$.

(ii) $W(m ; \underline{n})$ is a free $\mathcal{O}(m ; \underline{n})$-module with basis $\left\{D_{1}, \ldots, D_{m}\right\}$.

(iii) The elements in $\left\{x^{(a)} D_{i} \mid 0 \leq a_{i}<p^{n_{i}}, \quad 1 \leq i \leq m\right\}$ determine a basis for $W(m ; \underline{n})$ so that $\operatorname{dim} W(m ; \underline{n})=m p^{n_{1}+\cdots+n_{m}}$.

The divided power algebra is $\mathbb{Z}$-graded by the subspaces $\mathcal{O}(m)_{k}=$ $\operatorname{span}_{\mathbb{F}}\left\{x^{(a)}|| a \mid:=\sum_{j=1}^{m} a_{j}=k\right\}$. Thus $\mathcal{O}(m)=\bigoplus_{k=0}^{\infty} \mathcal{O}(m)_{k}$, and correspondingly, $\mathcal{O}(m ; \underline{n})=\bigoplus_{k=0}^{r+1} \mathcal{O}(m ; \underline{n})_{k}$ where $r=p^{n_{1}}+\cdots+p^{n_{m}}-m-1$. The associated Lie algebras $W(m)$ and $W(m ; \underline{n})$ inherit a grading by assigning

$$
W(m)_{k}=\bigoplus_{j=1}^{m} \mathcal{O}(m)_{k+1} D_{j} \text { and } W(m ; \underline{n})_{k}=\bigoplus_{j=1}^{m} \mathcal{O}(m ; \underline{n})_{k+1} D_{j} .
$$

In particular,

$$
W(m ; \underline{n})=\bigoplus_{j=-1}^{r} W(m ; \underline{n})_{j}
$$

is a depth-one simple Lie algebra of height $r$.

Observe that the null component $W(m ; \underline{n})_{0}=W(m)_{0}$ has the elements $x_{i}^{(1)} D_{j}(1 \leq i, j \leq m)$ as a basis. Moreover,

$$
\left[x_{i}^{(1)} D_{j}, x_{k}^{(1)} D_{\ell}\right]=\delta_{j, k} x_{i}^{(1)} D_{\ell}-\delta_{i, \ell} x_{k}^{(1)} D_{j} .
$$

Therefore, it is evident that the null component is isomorphic to $\mathfrak{g l}_{m}$ via the isomorphism that takes $x_{i}^{(1)} D_{j}$ to the matrix unit $E_{i, j}$.

The derivation

$$
\mathfrak{D}_{1}:=\sum_{j=1}^{m} x_{i}^{(1)} D_{i}
$$

(which corresponds to the identity matrix in $\mathfrak{g l}_{m}$ ) plays a special role in $W(m)$, as it is the "degree derivation":

$$
\mathfrak{D}_{1}\left(x^{(a)}\right)=\sum_{j=1}^{m} x_{j}^{(1)} D_{j}\left(x^{(a)}\right)=\left(\sum_{j=1}^{m} a_{j}\right) x^{(a)}=\operatorname{deg}\left(x^{(a)}\right) x^{(a)} .
$$

From the relation 


$$
[f D, g E]=f D(g) E-g E(f) D+f g[D, E],
$$

which holds for all $D, E \in W(m)$ and $f, g \in \mathcal{O}(m)$, we have as a special case,

$$
\left[\mathfrak{D}_{1}, x^{(a)} D_{j}\right]=\mathfrak{D}_{1}\left(x^{(a)}\right) D_{j}+x^{(a)}\left[\mathfrak{D}_{1}, D_{j}\right]=\left(\operatorname{deg}\left(x^{(a)}\right)-1\right) x^{(a)} D_{j},
$$

so that $\operatorname{ad} \mathfrak{D}_{1}$ acts as multiplication by the scalar $\ell$ on $W(m)_{\ell}$ for each $\ell$; that is to say, ad $\mathfrak{D}_{1}$ is the degree derivation on $W(m)$.

Now it follows from

$$
\left[x_{i}^{(1)} D_{j}, D_{k}\right]=-\delta_{i, k} D_{j}
$$

that $W(m)_{-1}=W(m ; \underline{n})_{-1}=\operatorname{span}_{\mathbb{F}}\left\{D_{1}, \ldots, D_{m}\right\}$ can be identified with the dual module of the natural $m$-dimensional module $V$ for $\mathfrak{g l}_{m}$. Hence it is isomorphic to the space $V^{*}$ of $1 \times m$ matrices over $\mathbb{F}$ with the $\mathfrak{g l}_{m}$-action given by $y . v=-v y$ (matrix multiplication) for all $v \in V^{*}$ and $y \in \mathfrak{g l}_{m}$.

The mapping $\varphi: W(m)_{0} \rightarrow \mathfrak{g l}\left(\mathcal{O}(m)_{1}\right)$ given by $\varphi(D)(f)=D(f)$ affords a representation of $W(m)_{0}$ (hence also of $W(m ; \underline{n})_{0}$ for any $m$-tuple $\underline{n}$ ). Moreover, relative to the standard basis $\left\{x_{1}^{(1)}, \ldots, x_{m}^{(1)}\right\}$ of $\mathcal{O}(m)_{1}$, the matrix of $\varphi\left(x_{i}^{(1)} D_{j}\right)$ is exactly the matrix unit $E_{i, j}$.

\subsection{Special Lie algebras of Cartan type (the $S$ series)}

The Lie algebra $S(m ; \underline{n})$ (resp. $S(m))$ is the subalgebra of $W(m ; \underline{n})$ (resp. $W(m))$ of derivations whose divergence is zero. Thus, if $D=\sum_{i=1}^{m} f_{i} D_{i}$, then

$$
\operatorname{div}(D):=\sum_{i=1}^{m} D_{i}\left(f_{i}\right)=0
$$

Observe that

$$
\begin{aligned}
\operatorname{div}\left(\left[f D_{i}, g D_{j}\right]\right) & =\operatorname{div}\left(f D_{i}(g) D_{j}-D_{j}(f) g D_{i}\right) \\
& =D_{j}(f) D_{i}(g)+f D_{j} D_{i}(g)-D_{i} D_{j}(f) g-D_{j}(f) D_{i}(g) \\
& =f D_{i}\left(D_{j}(g)\right)-g D_{j}\left(D_{i}(f)\right) \\
& =f D_{i}\left(\operatorname{div}\left(g D_{j}\right)\right)-g D_{j}\left(\operatorname{div}\left(f D_{i}\right)\right)
\end{aligned}
$$

so summing over such terms will show that the relation

$$
\operatorname{div}([D, E])=D(\operatorname{div}(E))-E(\operatorname{div}(D))
$$

holds for all $D, E \in W(m)$. From that it is apparent that $S(m ; \underline{n})$ (resp. $S(m))$ is indeed a subalgebra of $W(m ; \underline{n})$ (resp. $W(m))$. 
To better understand the structure of $S(m ; \underline{n})$, we introduce mappings $D_{i, j}: \mathcal{O}(m ; \underline{n}) \rightarrow W(m ; \underline{n})$ defined by

$$
D_{i, j}(f)=D_{j}(f) D_{i}-D_{i}(f) D_{j}, \quad 1 \leq i, j \leq m .
$$

It is easy to check that the image of $D_{i, j}$ lies in $S(m ; \underline{n})$. Moreover, $D_{i, i}=0$ and $D_{j, i}=-D_{i, j}$.

Lemma 2.32. (Compare [SF, Lem. 3.2] for (a) and (b).)

(a) For $D=\sum_{i=1}^{m} f_{i} D_{i}$ and $E=\sum_{j=1}^{m} g_{j} D_{j}$ in $S(m ; \underline{n})$,

$$
\left[\sum_{i=1}^{m} f_{i} D_{i}, \sum_{j=1}^{m} g_{j} D_{j}\right]=\sum_{i, j=1}^{n} D_{j, i}\left(f_{i} g_{j}\right)
$$

(b) $\left[D_{k}, D_{i, j}(f)\right]=D_{i, j}\left(D_{k}(f)\right)$ for all $1 \leq i, j, k \leq m$.

(c) For $1 \leq i, j, k, \ell \leq m$ we have

$$
\begin{aligned}
{\left[D_{i, j}(f), D_{k, \ell}(g)\right]=} & D_{i, \ell}\left(D_{j}(f) D_{k}(g)\right)-D_{j, \ell}\left(D_{i}(f) D_{k}(g)\right) \\
& -D_{k, j}\left(D_{i}(f) D_{\ell}(g)\right)+D_{k, i}\left(D_{j}(f) D_{\ell}(g)\right) .
\end{aligned}
$$

Proof. We know that

$$
[D, E]=\left[\sum_{i=1}^{m} f_{i} D_{i}, \sum_{j=1}^{m} g_{j} D_{j}\right]=\sum_{i, j=1}^{m} f_{i} D_{i}\left(g_{j}\right) D_{j}-g_{j} D_{j}\left(f_{i}\right) D_{i}
$$

from the general expression (2.27) for the Lie bracket. Then using the fact that $\operatorname{div}(D)=0=\operatorname{div}(E)$, we can see this product equals

$$
\begin{aligned}
\sum_{i, j=1}^{m}\left(f_{i} D_{i}\left(g_{j}\right)\right. & \left.+D_{i}\left(f_{i}\right) g_{j}\right) D_{j}-\sum_{i, j=1}^{m}\left(g_{j} D_{j}\left(f_{i}\right)+D_{j}\left(g_{j}\right) f_{i}\right) D_{i} \\
& =\sum_{i, j=1}^{m} D_{i}\left(f_{i} g_{j}\right) D_{j}-D_{j}\left(f_{i} g_{j}\right) D_{i} \\
& =\sum_{i, j=1}^{m} D_{j, i}\left(f_{i} g_{j}\right) .
\end{aligned}
$$

Part (b) is a simple consequence of the fact that

$$
\left[D_{k}, D_{i, j}(f)\right]=D_{k}\left(D_{j}(f)\right) D_{i}-D_{k}\left(D_{i}(f)\right) D_{j}=D_{i, j}\left(D_{k}(f)\right) .
$$

The verification of the identity in (c) is left as an exercise for the reader. The left side is the sum of 8 terms. When expanded out, the right side is the sum of 16 terms, 8 of which match with the terms on the left, and 8 of which pairwise sum to 0 . 
The subalgebra $S(m ; \underline{n})$ is not simple. However, its commutator ideal $S(m ; \underline{n})^{(1)}$ is, and we have the following.

Theorem 2.33. (Compare [SF, Prop. 3.3, Thm. 3.5, and Thm. 3.7].) Suppose $m \geq 3$.

(i) $S(m ; \underline{n})^{(1)}$ is the subalgebra of $S(m ; \underline{n})$ generated by the elements $D_{i, j}\left(x^{(a)}\right)$ where $0 \leq a_{k}<p^{n_{k}}$ for $1 \leq k \leq m$ and $1 \leq i, j \leq m$.

(ii) $S(m ; \underline{n})^{(1)}$ is simple.

(iii) $S(m ; \underline{n})^{(1)}=\bigoplus_{j=-1}^{s}\left(S(m ; \underline{n})^{(1)}\right)_{j}$ where $s=\left(\sum_{j=1}^{m} p^{n_{j}}\right)-m-2$ and $\left(S(m ; \underline{n})^{(1)}\right)_{j}=S(m ; \underline{n})^{(1)} \cap W(m ; \underline{n})_{j}$.

(iv) $S(m ; \underline{n})=S(m ; \underline{n})^{(1)} \oplus \bigoplus_{j=1}^{m} \mathbb{F} x^{\left(\tau-\left(p^{n_{j}}-1\right) \epsilon_{j}\right)} D_{j}$, where $\epsilon_{j}$ is the $m$-tuple with 1 in slot $j$ and 0 elsewhere, and

$$
\tau=\tau(\underline{n})=\left(p^{n_{1}}-1, \ldots, p^{n_{m}}-1\right) .
$$

We refer to the Lie algebras $S(m ; \underline{n})^{(1)}$ as the simple special Lie algebras of Cartan type. More generally, each of the Lie algebras $S(m ; \underline{n}), S(m)$, or $S(m ; \underline{n})^{(1)}$ is said to be a special Cartan type Lie algebra.

As $D_{i, j}\left(x_{j}^{(1)}\right)=D_{i}$, we have $\left(S(m ; \underline{n})^{(1)}\right)_{-1}=W(m ; \underline{n})_{-1}$. The derivations $D_{j, i}\left(x_{i}^{(2)}\right)=x_{i}^{(1)} D_{j}$ belong to $\left(S(m ; \underline{n})^{(1)}\right)_{0}$ for all $i \neq j$, as do the derivations $D_{i, i+1}\left(x_{i}^{(1)} x_{i+1}^{(1)}\right)=x_{i}^{(1)} D_{i}-x_{i+1}^{(1)} D_{i+1}$ for $i=1, \ldots, m-1$. Thus, $\operatorname{dim}\left(S(m ; \underline{n})^{(1)}\right)_{0} \geq m^{2}-1$. But the derivations $x_{i}^{(1)} D_{i}$ do not belong to $\left(S(m ; \underline{n})^{(1)}\right)_{0}$, as they fail the divergence zero test. Thus, $\operatorname{dim}\left(S(m ; \underline{n})^{(1)}\right)_{0}=$ $m^{2}-1$, and it is clear that $\left(S(m ; \underline{n})^{(1)}\right)_{0} \cong \mathfrak{s l}_{m}$.

There is a Lie algebra closely aligned to $S(m ; \underline{n})$ obtained by adjoining the degree derivation $\mathfrak{D}_{1}=\sum_{j=1}^{m} x_{i}^{(1)} D_{i}$ to the null component. As $\left[\mathfrak{D}_{1}, E\right]=k E$ for all $E \in W(m ; \underline{n})_{k}$ (compare (2.28)), the result of adding the degree derivation to $S(m ; \underline{n})$ is a Lie algebra, which we denote $C S(m ; \underline{n})$, whose null component is isomorphic to $\mathfrak{g l}_{m}$.

\subsection{Hamiltonian Lie algebras of Cartan type (the $H$ series)}

In this section, we will introduce a third series of Cartan type Lie algebras, but first some notation is required. Set

$$
\begin{aligned}
\sigma(j) & = \begin{cases}1 & \text { if } 1 \leq j \leq m \\
-1 & \text { if } m+1 \leq j \leq 2 m,\end{cases} \\
j^{\prime} & = \begin{cases}j+m & \text { if } 1 \leq j \leq m \\
j-m & \text { if } m+1 \leq j \leq 2 m .\end{cases}
\end{aligned}
$$


Set

$$
\begin{array}{r}
H(2 m ; \underline{n}):=\left\{D=\sum_{i=1}^{2 m} f_{i} D_{i} \in W(2 m ; \underline{n}) \mid \sigma\left(j^{\prime}\right) D_{i}\left(f_{j^{\prime}}\right)=\sigma\left(i^{\prime}\right) D_{j}\left(f_{i^{\prime}}\right),\right. \\
1 \leq i, j \leq 2 m\} .
\end{array}
$$

It can be argued that $H(2 m ; \underline{n})=W(2 m ; \underline{n}) \cap H(2 m)$, where $H(2 m)$ is the subalgebra of $W(2 m)$ consisting of all the derivations which satisfy $D\left(\omega_{H}\right)=$ 0 , where $\omega_{H}$ is the differential form given by

$$
\omega_{H}=\sum_{j=1}^{m} d x_{j} \wedge d x_{j+m}
$$

where $x_{j}=x_{j}^{(1)}$ for all $j$. In fact, the defining condition in (2.36) is equivalent to the statement that $D\left(\omega_{H}\right)=0$.

To gain further insight into the structure of $H(2 m ; \underline{n})$, we define

$$
D_{H}: \mathcal{O}(2 m) \rightarrow W(2 m), \quad D_{H}(f)=\sum_{j=1}^{2 m} \sigma(j) D_{j}(f) D_{j^{\prime}}
$$

and denote the image of the map $D_{H}$ restricted to $\mathcal{O}(2 m ; \underline{n})$ by $\tilde{H}(2 m ; \underline{n})$. The elements $D_{H}(f)$ belong to $H(2 m ; \underline{n})$ for all $f \in \mathcal{O}(2 m ; \underline{n})$, but the image of $D_{H}$ is proper, as the derivations $x_{j}^{\left(p^{n_{j}}-1\right)} D_{j^{\prime}}$ for $1 \leq j \leq 2 m$ belong to $H(2 m ; \underline{n})$, but fail to lie in the image.

Theorem 2.38. (See [SF, Lem. 4.1 and Thm. 4.5].)

(i) Let $D=\sum_{i=1}^{2 m} f_{i} D_{i}$ and $E=\sum_{j=1}^{2 m} g_{j} D_{j}$ be elements of $H(2 m ; \underline{n})$. Then

$$
[D, E]=D_{H}(u) \quad \text { where } \quad u=\sum_{j=1}^{2 m} \sigma(j) f_{j} g_{j^{\prime}} .
$$

(ii) $\tilde{H}(2 m ; \underline{n}) \supseteq H(2 m ; \underline{n})^{(1)}$.

(iii) $H(2 m ; \underline{n})^{(2)}$ is a simple Lie algebra with basis

$$
\left\{D_{H}\left(x^{(a)}\right) \mid a \neq(0, \ldots, 0) \text { and } a \neq\left(p^{n_{1}}-1, \ldots, p^{n_{2 m}}-1\right)\right\} .
$$

Thus,

$$
\operatorname{dim} H(2 m ; \underline{n})^{(2)}=p^{n_{1}+\cdots+n_{2 m}}-2 .
$$


(iv) For all $f, g \in \mathcal{O}(2 m ; \underline{n})$,

$$
\begin{aligned}
{\left[D_{H}(f), D_{H}(g)\right] } & =D_{H}\left(D_{H}(f)(g)\right) \\
& =D_{H}\left(\sum_{j=1}^{2 m} \sigma(j) D_{j}(f) D_{j^{\prime}}(g)\right)
\end{aligned}
$$

Some comments are in order. First, it follows from assertion (i) that $H(2 m ; \underline{n})^{(1)}$ is contained in $\tilde{H}(2 m ; \underline{n})$, and hence that $\tilde{H}(2 m ; \underline{n})$ is an ideal of $H(2 m ; \underline{n})$. The formula in (iv) is a consequence of (i), since

$$
\begin{aligned}
{\left[D_{H}(f), D_{H}(g)\right] } & =D_{H}(u) \quad \text { where } \\
u & =\sum_{j=1}^{2 m} \sigma(j) \sigma\left(j^{\prime}\right) D_{j^{\prime}}(f) \sigma(j) D_{j}(g) \\
& =\sum_{j=1}^{2 m} \sigma\left(j^{\prime}\right) D_{j^{\prime}}(f) D_{j}(g)=D_{H}(f)(g) .
\end{aligned}
$$

As special instances of that relation we have,

$$
\begin{aligned}
(2.41)\left[D_{H}\left(x^{(a)}\right), D_{H}\left(x_{j^{\prime}}^{(1)}\right)\right] & =\sigma(j) D_{H}\left(x^{\left(a-\epsilon_{j}\right)}\right) \\
{\left[D_{H}\left(x^{(a)}\right), D_{H}\left(x^{(b)}\right)\right] } & =\sum_{j=1}^{2 m} \sigma(j)\left(\begin{array}{c}
a+b-\epsilon_{j}-\epsilon_{j^{\prime}} \\
a-\epsilon_{j}
\end{array}\right) D_{H}\left(x^{\left(a+b-\epsilon_{j}-\epsilon_{j^{\prime}}\right)}\right) .
\end{aligned}
$$

From the first equation in $(2.41)$, we can see that $D_{H}\left(x^{(c)}\right) \in H(2 m ; \underline{n})^{(1)}$ for all $c \neq\left(p^{n_{1}}-1, \ldots, p^{n_{2 m}}-1\right)$. The coefficient of $D_{H}\left(x^{\left(a+b-\epsilon_{j}-\epsilon_{j^{\prime}}\right)}\right)$ in the second equation for $1 \leq j \leq m$ is

$$
\left(\begin{array}{c}
a+b-\epsilon_{j}-\epsilon_{j^{\prime}} \\
a-\epsilon_{j}
\end{array}\right)-\left(\begin{array}{c}
a+b-\epsilon_{j}-\epsilon_{j^{\prime}} \\
a-\epsilon_{j^{\prime}}
\end{array}\right) .
$$

If

$$
\tau=\tau(\underline{n}):=\left(p^{n_{1}}-1, \ldots, p^{n_{2 m}}-1\right),
$$

then $\left(\begin{array}{l}\tau \\ c\end{array}\right)=(-1)^{|c|}$ for all $2 m$-tuples $c$ such that $0 \leq c \leq \tau$. Now if $\tau=a+b-\epsilon_{j}-\epsilon_{j^{\prime}}$ for some $2 m$-tuples $a, b$, then $0 \leq a-\epsilon_{j}, a-\epsilon_{j^{\prime}} \leq \tau$ and $\left(\begin{array}{c}\tau \\ a-\epsilon_{j}\end{array}\right)-\left(\begin{array}{c}\tau \\ a-\epsilon_{j^{\prime}}\end{array}\right)=(-1)^{\left|a-\epsilon_{j}\right|}-(-1)^{\left|a-\epsilon_{j^{\prime}}\right|}=0$. Thus,

$$
\left[D_{H}\left(x^{(a)}\right), D_{H}\left(x^{(b)}\right)\right] \in \operatorname{span}_{\mathbb{F}}\left\{D_{H}\left(x^{(c)}\right) \mid 0 \leq c<\tau\right\} .
$$


As a consequence,

$$
\tilde{H}(2 m ; \underline{n})^{(1)}=\tilde{H}(2 m ; \underline{n})^{(2)}=\cdots=\operatorname{span}_{\mathbb{F}}\left\{D_{H}\left(x^{(c)}\right) \mid 0 \leq c<\tau\right\} .
$$

Because $\tilde{H}(2 m ; \underline{n})^{(1)} \supseteq H(2 m ; \underline{n})^{(2)} \supseteq \tilde{H}(2 m ; \underline{n})^{(2)}=\tilde{H}(2 m ; \underline{n})^{(1)}$, equality must hold, and $H(2 m ; \underline{n})^{(2)}=\operatorname{span}_{\mathbb{H}}\left\{D_{H}\left(x^{(c)}\right) \mid 0 \leq c<\tau\right\}$.

We may define a version of the ordinary Poisson bracket on $\mathcal{O}(2 m ; \underline{n})$ by

$$
\{f, g\}=\sum_{i=1}^{m} D_{i}(f) D_{i^{\prime}}(g)-D_{i^{\prime}}(f) D_{i}(g) .
$$

Note that

$$
\{f, g\}=\sum_{j=1}^{2 m} \sigma(j) D_{j}(f) D_{j^{\prime}}(g)=D_{H}(f)(g) .
$$

From this and Theorem 2.38 (iv) we see that $\overline{\mathcal{O}}(2 m ; \underline{n}):=\mathcal{O}(2 m ; \underline{n}) / \mathbb{F} 1$ is a Lie algebra under the Poisson bracket, and its derived algebra $\overline{\mathcal{O}}(2 m ; \underline{n})^{(1)}$ is isomorphic to $H(2 m ; \underline{n})^{(2)}$.

The Lie algebra $H(2 ; \underline{n})^{(2)}$ is exactly the Lie algebra $S(2 ; \underline{n})^{(1)}$. It is for that reason we have excluded the $m=2$ case from Theorem 2.33.

The degree derivation $\mathfrak{D}_{1}=\sum_{j=1}^{2 m} x_{j}^{(1)} D_{j}$ may be adjoined to the Lie algebra $H(2 m ; \underline{n})$ or $H(2 m ; \underline{n})^{(2)}$ to produce a larger Lie algebra. In the first case, we will write $C \bar{H}(2 m ; \underline{n})$; while in the second we will write $H(2 m ; \underline{n})^{(2)} \oplus \mathbb{F} \mathfrak{D}_{1}$ to avoid possible confusion with $(C H(2 m ; \underline{n}))^{(2)}$, which is just the algebra $H(2 m ; \underline{n})^{(1)}$ itself.

The derivations

$$
Q_{j}:=D_{H}\left(x_{j}^{\left(p^{n_{j}}\right)}\right)=\sigma(j) x_{j}^{\left(p^{n_{j}}-1\right)} D_{j^{\prime}}, \quad 1 \leq j \leq 2 m,
$$

belong to $H(2 m ; \underline{n})$, and expressions for their products may be derived using (iv) of Theorem 2.38:

$$
\begin{aligned}
{\left[Q_{i}, Q_{j}\right] } & =\delta_{i^{\prime}, j} \sigma(i) D_{H}\left(x_{i}^{\left(p^{n_{i}}-1\right)} x_{i^{\prime}}^{\left(p^{n_{i^{\prime}}}-1\right)}\right), \\
{\left[Q_{i}, D_{H}(f)\right] } & =D_{H}(g), \quad \text { where } g=\sigma(i) x_{i}^{\left(p^{\left.n_{i}-1\right)}\right.} D_{i^{\prime}}(f) .
\end{aligned}
$$

We can now state

Proposition 2.48. ([KS, Chap. 1, Sec. 6, Prop. 1]) The Lie algebra $H(2 m ; \underline{n})$ is spanned over $\mathbb{F}$ by the derivations $D_{H}(f), f \in \mathcal{O}(2 m ; \underline{n})$, as in (2.37), along with the derivations $Q_{j}, 1 \leq j \leq 2 m$, as in (2.45). Their products are given by (2.39), (2.46), and (2.47). 
The simple Lie algebra $H(2 m ; \underline{n})^{(2)}$ of Hamiltonian type inherits a grading from $W(2 m ; \underline{n})$ :

$$
\begin{aligned}
H(2 m ; \underline{n})^{(2)} & =\bigoplus_{j=-1}^{r}\left(H(2 m ; \underline{n})^{(2)}\right)_{j} \quad \text { where } \\
\left(H(2 m ; \underline{n})^{(2)}\right)_{j} & =H(2 m ; \underline{n})^{(2)} \cap W(2 m ; \underline{n})_{j}=D_{H}\left(\mathcal{O}(2 m ; \underline{n})_{j+2}\right), \quad \text { and } \\
r & =p^{n_{1}}+\cdots+p^{n_{2 m}}-2 m-3 .
\end{aligned}
$$

Thus, the null component $\left(H(2 m ; \underline{n})^{(2)}\right)_{0}$ is spanned by the elements

$$
D_{H}\left(x_{i}^{(1)} x_{j}^{(1)}\right)=\sigma(j) x_{i}^{(1)} D_{j^{\prime}}+\sigma(i) x_{j}^{(1)} D_{i^{\prime}} .
$$

Under the representation $\varphi: W(2 m)_{0} \rightarrow \mathfrak{g l}\left(\mathcal{O}(2 m)_{1}\right), \varphi(D)(f)=D(f)$, we see that the matrix of $\varphi\left(D_{H}\left(x_{i}^{(1)} x_{j}^{(1)}\right)\right)$ relative to the basis $\left\{x_{1}^{(1)}, \ldots, x_{2 m}^{(1)}\right\}$ is $\sigma(j) E_{i, j^{\prime}}+\sigma(i) E_{j, i^{\prime}}$. As those matrices span the symplectic Lie algebra $\mathfrak{s p}_{2 m}$, we have $\left(H(2 m ; \underline{n})^{(2)}\right)_{0} \cong \mathfrak{s p}_{2 m}$. Moreover, $\left(H(2 m ; \underline{n})^{(2)}\right)_{-1}$ is the dual $V^{*}$ of the natural $2 m$-dimensional module $V$ for $\mathfrak{s p}_{2 m}$, but $V^{*} \cong V$ as $\mathfrak{s p}_{2 m}$-modules. It is well-known (and can be seen from (2.50) and (2.40)) that

$$
\mathfrak{s p}_{2 m} \cong S^{2}(V)
$$

as $\mathfrak{s p}_{2 m}$-modules.

Finally, we note that the Lie algebra $C H(2 m ; \underline{n})$ also inherits a grading from $W(2 m ; \underline{n})$. The null component $C H(2 m ; \underline{n})_{0}$ is a one-dimensional central extension of $\left(H(2 m ; \underline{n})^{(2)}\right)_{0}$ by the degree derivation; thus it is a one-dimensional central extension of $\mathfrak{s p}_{2 m}$, which we denote by $\mathfrak{c s p}_{2 m}$.

\subsection{Contact Lie algebras of Cartan type (the $K$ series)}

In defining the final series of Cartan type Lie algebras, we suppose $\sigma(j)$ and $j^{\prime}$ are as in (2.34) for $j=1, \ldots, 2 m$. Our approach will follow [SF, Sec. 4.5] (compare also [KS, Sec. 7.1]). For $f \in \mathcal{O}(2 m+1)$, let

$$
\begin{gathered}
D_{K}(f):=\sum_{i=1}^{2 m+1} f_{i} D_{i}, \quad \text { where } \\
f_{i}=x_{i}^{(1)} D_{2 m+1}(f)+\sigma\left(i^{\prime}\right) D_{i^{\prime}}(f), \quad 1 \leq i \leq 2 m, \\
f_{2 m+1}=\Delta(f):=2 f-\sum_{j=1}^{2 m} x_{j}^{(1)} D_{j}(f) .
\end{gathered}
$$


In particular,

$$
\begin{aligned}
D_{K}(1) & =2 D_{2 m+1} \\
D_{K}\left(x_{j}^{(1)}\right) & =\sigma(j) D_{j^{\prime}}+x_{j}^{(1)} D_{2 m+1} \quad 1 \leq j \leq 2 m \\
D_{K}\left(x_{2 m+1}^{(1)}\right) & =\sum_{i=1}^{2 m} x_{i}^{(1)} D_{i}+2 x_{2 m+1}^{(1)} D_{2 m+1} .
\end{aligned}
$$

It follows (compare Proposition 2.61 below) that for all $f, g \in \mathcal{O}(2 m+1)$,

$$
\begin{aligned}
& {\left[D_{K}(f), D_{K}(g)\right]=D_{K}(u) \quad \text { where }} \\
& \quad u=\Delta(f) D_{2 m+1}(g)-\Delta(g) D_{2 m+1}(f)+\{f, g\} \text { and } \\
& \{f, g\}=\sum_{j=1}^{2 m} \sigma(j) D_{j}(f) D_{j^{\prime}}(g) \quad \text { as in }(2.44) .
\end{aligned}
$$

Thus, the elements $D_{K}(f), f \in \mathcal{O}(2 m+1)$, form a Lie subalgebra $K(2 m+1)$ of $W(2 m+1)$. They are precisely the derivations $D \in W(2 m+1)$ satisfying $D\left(\omega_{K}\right) \in \mathcal{O}(2 m+1) \omega_{K}$, where $\omega_{K}$ is the contact differential form

$$
\omega_{K}=d x_{2 m+1}+\sum_{j=1}^{2 m} \sigma(j) x_{j} d x_{j^{\prime}}
$$

and $x_{j}=x_{j}^{(1)}$ for all $j$.

REMARK 2.58. Note that the form used in [Wi1] and in [KS, Sec. 7.1] is $\omega_{K}^{\prime}=d x_{2 m+1}+\sum_{j=1}^{2 m} \sigma\left(j^{\prime}\right) x_{j} d x_{j^{\prime}}$, and so the formulas in those papers will be slightly different from the ones displayed in this work.

For each $(2 m+1)$-tuple $\underline{n}$, the Lie algebra $K(2 m+1 ; \underline{n})$ is by definition the intersection, $K(2 m+1 ; \underline{n})=K(2 m+1) \cap W(2 m+1 ; \underline{n})$.

The product in (2.57) can be expressed using the modified Poisson bracket

$$
\langle f, g\rangle:=\Delta(f) D_{2 m+1}(g)-\Delta(g) D_{2 m+1}(f)+\{f, g\} .
$$

Thus,

$$
\left[D_{K}(f), D_{K}(g)\right]=D_{K}(\langle f, g\rangle)
$$

Proposition 2.61. (Compare [SF, Sec. 4.5, Prop. 5.3].) Suppose for $a=\left(a_{1}, \ldots, a_{2 m+1}\right), a_{i} \in \mathbb{N}$, that $\|a\|:=|a|+a_{2 m+1}-2$. Then

(i) $\left\langle x^{(a)}, x^{(b)}\right\rangle=\left\{x^{(a)}, x^{(b)}\right\}$

$$
+\left(\|b\|\left(\begin{array}{c}
a+b-\epsilon_{2 m+1} \\
b
\end{array}\right)-\|a\|\left(\begin{array}{c}
a+b-\epsilon_{2 m+1} \\
a
\end{array}\right)\right) x^{\left(a+b-\epsilon_{2 m+1}\right)}
$$


(ii) $\left\langle 1, x^{(a)}\right\rangle=2 x^{\left(a-\epsilon_{2 m+1}\right)}$;

(iii) $\left\langle x_{j}^{(1)}, x^{(a)}\right\rangle=\sigma(j) x^{\left(a-\epsilon_{j^{\prime}}\right)}+\left(a_{j}+1\right) x^{\left(a+\epsilon_{j}-\epsilon_{2 m+1}\right)}, \quad 1 \leq j \leq 2 m$;

(iv) $\left\langle x_{2 m+1}^{(1)}, x^{(a)}\right\rangle=\|a\| x^{(a)}$;

(v) $\left\langle x_{i}^{(1)} x_{j}^{(1)}, x^{(a)}\right\rangle=\sigma(i) a_{j} x^{\left(a+\epsilon_{j}-\epsilon_{i^{\prime}}\right)}+\sigma(j) a_{i} x^{\left(a+\epsilon_{i}-\epsilon_{j^{\prime}}\right)}$,

(vi) $\left\langle x_{i}^{(1)} x_{i^{\prime}}^{(1)}, x^{(a)}\right\rangle=\left(a_{i^{\prime}}-a_{i}\right) x^{(a)}, \quad 1 \leq i \leq m$;

$$
1 \leq i, j \leq 2 m
$$

(vii) The spaces $K(2 m+1 ; \underline{n})_{j}=\operatorname{span}_{\mathbb{F}}\left\{D_{K}\left(x^{(a)}\right) \mid\|a\|=j\right\}$ give $a$ grading of the Lie algebra $K(2 m+1 ; \underline{n})$ such that

$$
K(2 m+1 ; \underline{n})=\bigoplus_{j=-2}^{r} K(2 m+1 ; \underline{n})_{j},
$$

where $r=p^{n_{1}}+\cdots+p^{n_{2 m}}+2 p^{n_{2 m+1}}-2 m-3$.

Theorem 2.62. (See [SF, Sec. 4.5, Thm. 5.5].) $K(2 m+1 ; \underline{n})^{(1)}$ is a simple Lie algebra, and

$K(2 m+1 ; \underline{n})^{(1)}= \begin{cases}K(2 m+1 ; \underline{n}) & \text { if } 2 m+4 \not \equiv 0 \quad \bmod p \\ \operatorname{span}_{\mathbb{F}}\left\{D_{K}\left(x^{(a)}\right) \mid a \neq \tau(\underline{n})\right\} & \text { if } 2 m+4 \equiv 0 \quad \bmod p,\end{cases}$

where $\tau(\underline{n})=\left(p^{n_{1}}-1, \ldots, p^{n_{2 m+1}}-1\right)$. Thus, $\operatorname{dim} K(2 m+1 ; \underline{n})^{(1)}$ has dimension $p^{n_{1}+\cdots+n_{2 m+1}}$ in the first case and dimension $p^{n_{1}+\cdots+n_{2 m+1}}-1$ in the second. Moreover, $K(2 m+1 ; \underline{n})^{(1)}$ is isomorphic to $\mathcal{O}(2 m+1 ; \underline{n})^{(1)}$, where $\mathcal{O}(2 m+1 ; \underline{n})$ is viewed as a Lie algebra under the product $f \times g \mapsto\langle f, g\rangle$.

From parts (ii)-(vi) of Proposition 2.61, we see that $K(2 m+1 ; \underline{n})_{-2}$ is spanned by $d_{2 m+1}:=D_{K}(1)=2 D_{2 m+1} ;$ and $K(2 m+1 ; \underline{n})_{-1}$ is spanned by the elements $d_{j}:=\sigma\left(j^{\prime}\right) D_{K}\left(x_{j^{\prime}}^{(1)}\right)=D_{j}+\sigma\left(j^{\prime}\right) x_{j^{\prime}}^{(1)} D_{2 m+1}$ for $j=1, \ldots, 2 m$. These elements satisfy the rule

$$
\left[d_{i}, d_{j}\right]=\delta_{i, j^{\prime}} \sigma(i) d_{2 m+1} \quad 1 \leq i, j \leq 2 m .
$$

The elements $D_{K}\left(x^{(a)}\right)$ with $\|a\|=0$ span the subalgebra $K(2 m+1 ; \underline{n})_{0}$, which is isomorphic to the Lie algebra $\mathfrak{c s p}_{2 m}=\mathfrak{s p}_{2 m} \oplus \mathbb{F} I$. The space $K(2 m+1 ; \underline{n})_{-1}$ is its natural $2 m$-dimensional module.

REMARK 2.64. For any of the simple Lie algebras $\mathfrak{g}=\bigoplus_{i} \mathfrak{g}_{i}$ of Cartan type discussed in Sections 2.8-2.11, the sum $\mathfrak{m}_{0}:=\bigoplus_{i>0} \mathfrak{g}_{i}$ is a maximal subalgebra of $\mathfrak{g}$ which is invariant under the automorphisms of $\mathfrak{g}$. This uniqueness property of $\mathfrak{m}_{0}$ is justification for calling the grading we have described in those sections the natural grading of $\mathfrak{g}$. Moreover, the spaces $m_{\ell}:=\bigoplus_{i \geq \ell} \mathfrak{g}_{i}$ for $\ell \geq-1$ afford a filtration $\mathfrak{g} \supseteq \mathfrak{m}_{-1} \supset \mathfrak{m}_{0} \supset \mathfrak{m}_{1} \supset \cdots$ of $\mathfrak{g}$, which is often referred to as the natural filtration (see for example, [St, Defn. 4.2.8].) 
In closing this subsection, we comment that very few of the simple Cartan type Lie algebras described above are restricted. In fact, the restricted ones may be characterized as having their defining $m$-tuple $\underline{n}=\underline{1}$, the tuple of all ones.

Proposition 2.65. (See [K3, Thm. 2] and [St, (7.2)].) Let $\mathfrak{g}$ be a restricted simple Lie algebra of Cartan type. Then $\mathfrak{g}$ isomorphic to one of the following: $W(m ; \underline{1})(m \geq 1), S(m ; \underline{1})^{(1)}(m \geq 3), H(2 m ; \underline{1})^{(2)}(m \geq 1)$, or $K(2 m+1 ; \underline{1})^{(1)}(m \geq 1)$.

\subsection{The Recognition Theorem with stronger hypotheses}

By imposing strong assumptions on the non-positive homogeneous components, we can deduce the following version of the Recognition Theorem. The next two chapters will be devoted to showing that these additional hypotheses must hold when the conditions of the Main Theorem are fulfilled. A similar result, phrased in the language of filtered Lie algebras, appears in [St, Cor. 5.5.3 (Weak Recognition Theorem)] for simple Lie algebras.

TheOREM 2.66. Let $\mathfrak{g}=\bigoplus_{j=-2}^{r} \mathfrak{g}_{j}$ be a finite-dimensional graded Lie algebra over an algebraically closed field $\mathbb{F}$ of characteristic $p>3$. Assume that:

(a) $\mathfrak{g}_{0}$ is isomorphic to $\mathfrak{g l}_{m}, \mathfrak{s l}_{m}, \mathfrak{s p}_{2 m}$, or $\mathfrak{c s p}_{2 m}=\mathfrak{s p}_{2 m} \oplus \mathbb{F} I$.

(b) $\mathfrak{g}_{-1}$ is a standard $\mathfrak{g}_{0}$-module (of dimension $m$ or $2 m$, depending on the simple classical Lie algebra $\left.\mathfrak{g}_{0}^{(1)}=\left[\mathfrak{g}_{0}, \mathfrak{g}_{0}\right]\right)$.

(c) If $\mathfrak{g}_{-2} \neq 0$, then it is one-dimensional and equals $\left[\mathfrak{g}_{-1}, \mathfrak{g}_{-1}\right]$, and $\mathfrak{g}_{0}$ is isomorphic to $\mathfrak{c s p}_{2 m}$.

(d) If $x \in \bigoplus_{j \geq 0} \mathfrak{g}_{j}$ and $\left[x, \mathfrak{g}_{-1}\right]=0$, then $x=0$ (transitivity);

(e) If $x \in \bigoplus_{j \geq 0} \mathfrak{g}_{-j}$ and $\left[x, \mathfrak{g}_{1}\right]=0$, then $x=0$ (1-transitivity).

Then either $\mathfrak{g}$ is a Cartan type Lie algebra with the natural grading:

$$
X(\mathfrak{m} ; \underline{n})^{(2)} \subseteq \mathfrak{g} \subseteq X(\mathfrak{m} ; \underline{n})
$$

where

$$
X=\left\{\begin{array}{lll}
W, S, \text { or } C S & \text { and } \quad \mathfrak{m}=m, \\
H \text { or } C H & \text { and } \quad \mathfrak{m}=2 m, \\
K & \text { and } \quad \mathfrak{m}=2 m+1
\end{array}\right.
$$

or $\mathfrak{g}$ is a classical simple Lie algebra:

a) $\mathfrak{g} \cong \mathfrak{s l}_{m+1} \hookrightarrow W(m ; \underline{n})$, or

b) $\mathfrak{g} \cong \mathfrak{s p}_{2(m+1)} \hookrightarrow K(2 m+1 ; \underline{n})$. 
Proof. By [St, Prop. 2.7.3], the Lie algebra $\mathfrak{g}$ is isomorphic to a subalgebra of a Witt Lie algebra of Cartan type. Then the theorem follows from [St, Lem. 5.2.3].

\subsection{3. $\mathfrak{g}_{\ell}$ as a $\mathfrak{g}_{0}$-module for Lie algebras $\mathfrak{g}$ of Cartan type}

Next we will investigate the structure of $\mathfrak{g}_{\ell}(\ell<p-1)$ as a $\mathfrak{g}_{0}$-module when $\mathfrak{g}$ is a depth-one Lie algebra of Cartan type. Here we will follow Section 10 of Chapter I of $[\mathbf{K S}]$. See also [St, Sec. 5.2] for related results. For this purpose, it is convenient to introduce the following derivations in $W(m)$ :

$$
\mathfrak{D}_{f}:=f \mathfrak{D}_{1} \quad \text { for } f \in \mathcal{O}(m),
$$

where $\mathfrak{D}_{1}=\sum_{j=1}^{m} x_{j}^{(1)} D_{j}$ is the degree derivation. Then $\operatorname{deg}\left(\mathfrak{D}_{f}\right)=\operatorname{deg}(f)$ for all homogeneous $f \in \mathcal{O}(m)$, and these derivations multiply according to the rule,

$$
\begin{aligned}
{\left[\mathfrak{D}_{f}, \mathfrak{D}_{g}\right] } & =\left[f \mathfrak{D}_{1}, g \mathfrak{D}_{1}\right] \\
& =\left(f \mathfrak{D}_{1}(g)-g \mathfrak{D}_{1}(f)\right) \mathfrak{D}_{1} \\
& =(\operatorname{deg}(g)-\operatorname{deg}(f)) f g \mathfrak{D}_{1}=(\operatorname{deg}(g)-\operatorname{deg}(f)) \mathfrak{D}_{f g} .
\end{aligned}
$$

In deriving this expression, we have applied the general relation

$$
[f D, g E]=f D(g) E-g E(f) D+f g[D, E]
$$

in (2.27). Recall from (2.28) that ad $\mathfrak{D}_{1}$ acts as multiplication by the scalar $\ell$ on $W(m)_{\ell}$ for each $\ell$. In particular, when $\mathfrak{g}=W(m)$ and $m=1$, then $\mathfrak{g}_{0}=\mathbb{F} \mathfrak{D}_{1}$, and for each $\ell \geq-1$, the space $\mathfrak{g}_{\ell}=\mathbb{F} x_{1}^{(\ell+1)} D_{1}$ is an irreducible $\mathfrak{g}_{0}$-module on which $\mathfrak{D}_{1}$ acts as multiplication by the scalar $\ell$. The $\mathfrak{g}_{0}$-module structure of $\mathfrak{g}_{\ell}$ for $m \geq 2$ is the topic of the next result.

Theorem 2.69. Assume $\mathfrak{g}=W(m)$ for $m \geq 2$, and let $\mathfrak{g}_{\ell}=W(m)_{\ell}$ for all $\ell \geq-1$. Let $\mathfrak{h}$ be the Cartan subalgebra of $\mathfrak{g}_{0}$ with basis $x_{j}^{(1)} D_{j}$ $(1 \leq j \leq m)$, and let $\varepsilon_{i}(1 \leq i \leq m)$ be the dual basis in $\mathfrak{h}^{*}$ so that $\varepsilon_{i}\left(x_{j}^{(1)} D_{j}\right)=\delta_{i, j}$. Let $\mathfrak{b}^{+}=\mathfrak{h} \oplus \mathfrak{n}^{+}$, where $\mathfrak{n}^{+}$is the $\mathbb{F}$-span of all $x_{i}^{(1)} D_{j}$ with $1 \leq i<j \leq m$, and set

$$
\begin{aligned}
\mathfrak{g}_{\ell}^{\dagger} & :=\left\{D \in \mathfrak{g}_{\ell} \mid \operatorname{div}(D)=0\right\} \\
\mathfrak{g}_{\ell}^{\sharp} & :=\operatorname{span}_{\mathbb{F}}\left\{\mathfrak{D}_{f}=f \sum_{j=1}^{m} x_{j}^{(1)} D_{j}=f \mathfrak{D}_{1} \mid \operatorname{deg}(f)=\ell\right\} .
\end{aligned}
$$

Then for $\ell \leq p-2$ we have: 
(i) $\mathfrak{g}_{\ell}^{\dagger}$ is an irreducible $\mathfrak{g}_{0}$-submodule of $\mathfrak{g}_{\ell}$ with a $\mathfrak{b}^{+}$-primitive vector of weight $(\ell+1) \varepsilon_{1}-\varepsilon_{m}$ when $m+\ell \neq \equiv 0 \bmod p$;

(ii) $\mathfrak{g}_{\ell}^{\sharp}$ is an irreducible $\mathfrak{g}_{0}$-submodule of $\mathfrak{g}_{\ell}$ with a $\mathfrak{b}^{+}$-primitive vector of weight $\ell \varepsilon_{1}$, and $\mathfrak{g}_{\ell}^{\sharp}$ is isomorphic as a $\mathfrak{g}_{0}$-module to $S^{\ell}(V)$, where $V=\mathcal{O}(m)_{1}$ is the natural $m$-dimensional module of $\mathfrak{g}_{0}$;

(iii) $\mathfrak{g}_{\ell}=\mathfrak{g}_{\ell}^{\sharp} \oplus \mathfrak{g}_{\ell}^{\dagger}$ when $m+\ell \not \equiv 0 \bmod p$;

(iv) When $m \geq 3$ and $m+\ell \equiv 0 \bmod p$, then $\mathfrak{g}_{\ell} \supset \mathfrak{g}_{\ell}^{\dagger} \supset \mathfrak{g}_{\ell}^{\sharp} \supset 0$ is the unique composition series of the $\mathfrak{g}_{0}$-module $\mathfrak{g}_{\ell}$;

(v) When $m=2$ and $m+\ell \equiv 0 \bmod p$ (i.e. $\ell=p-2$ ), then $\mathfrak{g}_{p-2}^{\dagger}$ is spanned modulo $\mathfrak{g}_{p-2}^{\sharp}$ by $x_{1}^{(p-1)} D_{2}$ and $x_{2}^{(p-1)} D_{1}$. Moreover, $\mathfrak{g}_{p-2}^{\dagger} / \mathfrak{g}_{p-2}^{\sharp} \cong L(0) \oplus L(0)$ and $\mathfrak{g}_{p-2} / \mathfrak{g}_{p-2}^{\dagger} \cong \mathfrak{g}_{p-2}^{\sharp} \cong L\left((p-2) \varpi_{1}\right) \cong$ $S^{p-2}(V)$ as modules for $\mathfrak{g}_{0}^{(1)} \cong \mathfrak{s l}_{2}$.

Proof. By (2.30) we have

$$
\begin{aligned}
\operatorname{div}\left(\left[x_{i}^{(1)} D_{j}, D\right]\right) & =x_{i}^{(1)} D_{j}(\operatorname{div}(D))-D\left(\operatorname{div}\left(x_{i}^{(1)} D_{j}\right)\right) \\
& =x_{i}^{(1)} D_{j}(\operatorname{div}(D))-D\left(\delta_{i, j} 1\right) \\
& =x_{i}^{(1)} D_{j}(\operatorname{div}(D))
\end{aligned}
$$

which implies that $\mathfrak{g}_{\ell}^{\dagger}$ is a $\mathfrak{g}_{0}$-submodule of $\mathfrak{g}_{\ell}$ for all $\ell$. This is also evident from the fact that $\mathfrak{g}_{0}=\mathfrak{g}_{0}^{(1)} \oplus \mathbb{F} \mathfrak{D}_{1}$, where $\mathfrak{g}_{0}^{(1)}=S(m)_{0}$, and $\mathfrak{g}_{\ell}^{\dagger}=S(m)_{\ell}$.

Applying (2.27), we obtain

$$
\begin{aligned}
{\left[x_{i}^{(1)} D_{j}, \mathfrak{D}_{f}\right] } & =x_{i}^{(1)}\left[D_{j}, \mathfrak{D}_{f}\right]-\mathfrak{D}_{f}\left(x_{i}^{(1)}\right) D_{j} \\
& =x_{i}^{(1)}\left[D_{j}, f \mathfrak{D}_{1}\right]-f \mathfrak{D}_{1}\left(x_{i}^{(1)}\right) D_{j} \\
& =x_{i}^{(1)} D_{j}(f) \mathfrak{D}_{1}+x_{i}^{(1)} f D_{j}-x_{i}^{(1)} f D_{j}=x_{i}^{(1)} D_{j}(f) \mathfrak{D}_{1} .
\end{aligned}
$$

Thus, $\mathfrak{g}_{\ell}^{\sharp}$ is also a $\mathfrak{g}_{0}$-submodule of $\mathfrak{g}_{\ell}$. Moreover, if $V=\mathcal{O}(m)_{1}$, then the calculation in $(2.73)$ shows that $\mathfrak{g}_{\ell}^{\sharp} \cong \mathcal{O}(m)_{\ell}=S^{\ell}(V)$ as $\mathfrak{g}_{0}$-modules via the identification $f \mapsto \mathfrak{D}_{f}$. The vector $\mathfrak{D}_{x_{1}^{(\ell)}}=x_{1}^{(\ell)} \mathfrak{D}_{1}$ has zero product with all $x_{i}^{(1)} D_{j}$ such that $i<j$, and $\left[x_{i}^{(1)} D_{i}, \mathfrak{D}_{x_{1}^{(\ell)}}\right]=\ell \delta_{i, 1} \mathfrak{D}_{x_{1}^{(\ell)}}$. Therefore, $\mathfrak{D}_{x_{1}^{(\ell)}}$ is a $\mathfrak{b}^{+}$-primitive vector of $\mathfrak{g}_{\ell}^{\sharp}$ and its weight is $\ell \varepsilon_{1}$ relative to the Cartan subalgebra $\mathfrak{h}$. Relative to $\mathfrak{g}_{0}^{(1)} \cong \mathfrak{s l}_{m}$ and its Cartan subalgebra $\mathfrak{h} \cap \mathfrak{g}_{0}^{(1)}$, the module $S^{\ell}(V)$ has a $\mathfrak{b}^{+}$-primitive vector $x_{1}^{(\ell)}$ of weight $\ell \varpi_{1}$. Since $\ell \leq p-2$, the irreducible $\mathfrak{s l}_{m}$-module $L\left(\ell \varpi_{1}\right)$ is isomorphic to the Weyl module $V\left(\ell \varpi_{1}\right)$, which has dimension given by Weyl's dimension formula 
(see $(2.11))$ so that

$$
\operatorname{dim} V\left(\ell \varpi_{1}\right)=\left(\begin{array}{c}
m+\ell-1 \\
\ell
\end{array}\right)=\operatorname{dim} S^{\ell}(V) .
$$

It follows that $\mathfrak{g}_{\ell}^{\sharp}$ is an irreducible $\mathfrak{g}_{0}^{(1)}$-module (hence an irreducible $\mathfrak{g}_{0^{-}}$ module) isomorphic to $S^{\ell}(V)$ for all $\ell \leq p-2$, as asserted in (ii) .

Consider the sequence of $\mathfrak{g}_{0}$-module maps,

$$
\begin{aligned}
\mathfrak{g}_{\ell} & \stackrel{\sim}{\rightarrow} S^{\ell+1}(V) \otimes V^{*} \rightarrow S^{\ell}(V) \\
f D_{k} & \mapsto f \otimes D_{k} \mapsto \operatorname{div}\left(f D_{k}\right)=D_{k}(f) .
\end{aligned}
$$

The kernel is $\mathfrak{g}_{\ell}^{\dagger}$. Since

$$
\operatorname{div}\left(\mathfrak{D}_{u}\right)=(m+\ell) u
$$

for all $u \in \mathcal{O}(m)_{\ell}$, we see that $\mathfrak{g}_{\ell}^{\sharp} \subset \mathfrak{g}_{\ell}^{\dagger}$ if $m+\ell \equiv 0$, and $\mathfrak{g}_{\ell}^{\sharp} \cap \mathfrak{g}_{\ell}^{\dagger}=0$ if $m+\ell \not \equiv 0 \bmod p$.

Suppose $x^{(a)} D_{k} \in \mathfrak{g}_{\ell}$ and set

$E_{j}:=\left(a_{j}+1\right) D_{k, j}\left(x^{\left(a+\epsilon_{j}\right)}\right)=\left(a_{j}+1\right) x^{(a)} D_{k}-x^{\left(a-\epsilon_{k}\right)} x_{j}^{(1)} D_{j}-\delta_{j, k} x^{(a)} D_{j} \in \mathfrak{g}_{\ell}^{\dagger}$.

Then

$$
\begin{aligned}
(m+\ell) x^{(a)} D_{k} & =\left(\sum_{j=1}^{m}\left(a_{j}+1\right)\right) x^{(a)} D_{k}-x^{(a)} D_{k} \\
& =\sum_{j=1}^{m} E_{j}+x^{\left(a-\epsilon_{k}\right)} \sum_{j=1}^{m} x_{j}^{(1)} D_{j} \\
& =\sum_{j=1}^{m} E_{j}+x^{\left(a-\epsilon_{k}\right)} \mathfrak{D}_{1} .
\end{aligned}
$$

Consequently, when $m+\ell \neq \equiv 0 \bmod p, \quad \mathfrak{g}_{\ell} \subseteq \mathfrak{g}_{\ell}^{\dagger}+\mathfrak{g}_{\ell}^{\sharp}$ so that $\mathfrak{g}_{\ell}=\mathfrak{g}_{\ell}^{\dagger} \oplus \mathfrak{g}_{\ell}^{\sharp}$ as asserted in (iii). We also see that the above map $x^{(a)} D_{k} \mapsto D_{k}\left(x^{(a)}\right)=$ $x^{\left(a-\epsilon_{k}\right)}$ is essentially (up to a factor of $\left.(m+\ell)^{-1}\right)$ the projection of $\mathfrak{g}_{\ell}$ onto the $\mathfrak{g}_{0}$-submodule $\mathfrak{g}_{\ell}^{\sharp} \cong S^{\ell}(V)$.

Recall that $\mathfrak{n}^{+}$is the $\mathbb{F}$-span of all $x_{i}^{(1)} D_{j}$ with $1 \leq i<j \leq m$, a maximal nilpotent subalgebra of $\mathfrak{g}_{0}^{(1)}$, and $\mathfrak{b}^{+}:=\mathfrak{h} \oplus \mathfrak{n}^{+}$. We claim that any $\mathfrak{b}^{+}$-primitive vector of $\mathfrak{g}_{\ell}$ is a scalar multiple of $x_{1}^{(\ell+1)} D_{m}$ or of $\mathfrak{D}_{x_{1}^{(\ell)}}$. The claim certainly holds when $\ell=0$ (see (2.73)). Assume that it holds for all $\ell<s$ where $1 \leq s \leq p-2$, and let $u$ be a $\mathfrak{b}^{+}$-primitive vector of $\mathfrak{g}_{s}$. Since $\left[\mathfrak{g}_{-1}, u\right] \neq 0$, by transitivity, $\left[D_{k}, u\right]$ is a $\mathfrak{b}^{+}$-primitive vector of $\mathfrak{g}_{s-1}$ for some $k \leq m$. In view of our assumption, this means that either $\left[D_{k}, u\right]=\zeta x_{1}^{(s)} D_{m}$ or $\left[D_{k}, u\right]=\zeta \mathfrak{D}_{x_{1}^{(s-1)}}$ where $\zeta \in \mathbb{F}^{\times}$. No generality will be lost by assuming that $\zeta=1$. 
Suppose $\left[D_{k}, u\right]=\mathfrak{D}_{x_{1}^{(s-1)}}$. If $k>1$, then

$u=x_{1}^{(s-1)} x_{k}^{(1)}\left(\sum_{i \neq k} x_{i}^{(1)} D_{i}+\frac{1}{2} x_{k}^{(1)} D_{k}\right)+u_{0}, \quad u_{0} \in \mathfrak{g}_{s} \cap \operatorname{ker} \operatorname{ad} D_{k}$.

From this it is immediate that

$$
\left[x_{k-1}^{(1)} D_{k}, u\right] \equiv x_{1}^{(s-1)} x_{k-1}^{(1)} \sum_{i \neq k} x_{i}^{(1)} D_{i} \quad \bmod \mathcal{O}(m) D_{k} .
$$

Since $\left[\mathfrak{n}^{+}, u\right]=0$, we have reached a contradiction, showing that $k=1$. Then

$$
u=s x_{1}^{(s+1)} D_{1}+x_{1}^{(s)} \sum_{i=2}^{m} x_{i}^{(1)} D_{i}+u_{0}^{\prime}, \quad u_{0}^{\prime} \in \mathfrak{g}_{s} \cap \operatorname{ker} \operatorname{ad} D_{1},
$$

implying

$$
\begin{aligned}
{\left[x_{1}^{(1)} D_{2}, u\right] } & =-s x_{1}^{(s+1)} D_{2}+x_{1}^{(s)} x_{1}^{(1)} D_{2}+x_{1}^{(1)}\left[D_{2}, u_{0}^{\prime}\right]-u_{0}^{\prime}\left(x_{1}^{(1)}\right) D_{2} \\
& =x_{1}^{(s+1)} D_{2}+x_{1}^{(1)} \sum_{i=1}^{m} f_{i} D_{i}+g D_{2}
\end{aligned}
$$

where $g$ and the $f_{i}$ belong to the subalgebra of $\mathcal{O}(m)$ generated by $x_{j}^{(r)}$ with $j \geq 2$ and $r \geq 0$. Since $s \geq 1$ it follows that $\left[x_{1}^{(1)} D_{2}, u\right] \neq 0$, a contradiction. We conclude that $\left[D_{k}, u\right]=x_{1}^{(s)} D_{m}$.

If $k>1$ then $u=x_{1}^{(s)} x_{k}^{(1)} D_{m}+u_{1}$ where $u_{1} \in \mathfrak{g}_{s} \cap \operatorname{ker}$ ad $D_{k}$. Then, for $i<k$,

$$
0=\left[x_{i}^{(1)} D_{k}, u\right]=x_{1}^{(s)} x_{i}^{(1)} D_{m}-u_{1}\left(x_{i}^{(1)}\right) D_{k} .
$$

This shows that $k=m$ and $u_{1}=a D_{m}+\sum_{i=1}^{m-1} x_{1}^{(s)} x_{i}^{(1)} D_{i}$ for some $a \in$ $\mathcal{O}(m)_{s+1}$ with $D_{m}(a)=0$. But then $u=a D_{m}+\mathfrak{D}_{x_{1}^{(s)}}$ implying $\left[\mathfrak{n}^{+}, a D_{m}\right]=$ 0 . As a consequence, $\left(x_{i}^{(1)} D_{j}\right)(a)=0$ whenever $1 \leq i<j \leq m-1$. From this it is easy to deduce that $a=\mu x_{1}^{(s+1)}$ for some $\mu \in \mathbb{F}$.

If $k=1$ then $u=x_{1}^{(s+1)} D_{m}+u_{1}^{\prime}$ where $u_{1}^{\prime} \in \mathfrak{g}_{s} \cap \operatorname{ker}$ ad $D_{1}$. Then, for $i>1$,

$$
0=\left[x_{1}^{(1)} D_{i}, u\right]=x_{1}^{(1)}\left[D_{i}, u_{1}^{\prime}\right]-u_{1}^{\prime}\left(x_{1}^{(1)}\right) D_{i} .
$$

Since $u_{1}^{\prime}\left(x_{1}^{(1)}\right) \in \mathcal{O}(m)$ does not involve $x_{1}^{(1)}$, it follows that $\left[D_{i}, u_{1}^{\prime}\right]=0$ for all $i$. But then $u_{1}^{\prime}=0$ by transitivity. This proves our claim.

Now assume $m \geq 3$. Set $G_{0}:=\mathrm{SL}(V)$, a simply connected algebraic $\mathbb{F}$-group, and identify $\operatorname{Lie}\left(G_{0}\right)$ with $\mathfrak{g}_{0}^{(1)}$. Let $T$ be the maximal torus of $G_{0}$ such that $\operatorname{Lie}(T)=\mathfrak{h}$, and let $B^{+}$be the Borel subgroup of $G_{0}$ with $\operatorname{Lie}\left(B^{+}\right)=\mathfrak{b}^{+}$. By abuse of notation, we will identify the weights $\mu$ of $T$ with their differentials $(\mathrm{d} \mu)_{e} \in \mathfrak{h}^{*}$. In particular, the fundamental weights in $X(T)_{+}$will be denoted by $\varpi_{1}, \varpi_{2}, \ldots, \varpi_{m-1}$ (this will cause no confusion). Note that $\mathfrak{g}_{\ell}$ is isomorphic to $S^{\ell+1}(V) \otimes V^{*}$, which is a rational $G_{0}$-module. Since $\ell \leq p-2$, it is easy to see that $X_{+}\left(S^{\ell+1}(V) \otimes V^{*}\right) \subset X_{1}(T)$. So the 
$G_{0}$-module $\mathfrak{g}_{\ell}$ satisfies all the conditions of Proposition 2.15. It follows that every $\mathfrak{g}_{0}^{(1)}$-submodule of $\mathfrak{g}_{\ell}$ is $G_{0}$-stable.

We claim that the $\mathfrak{g}_{0}^{(1)}$-submodule of $\mathfrak{g}_{\ell}$ generated by $x_{1}^{(\ell+1)} D_{m}$ coincides with $\mathfrak{g}_{\ell}^{\dagger}$. Let us denote this submodule by $\mathfrak{g}_{\ell}^{\prime}$ and suppose for a contradiction that $\mathfrak{g}_{\ell}^{\prime} \neq \mathfrak{g}_{\ell}^{\dagger}$. Since we are assuming $m \geq 3$, Theorem 2.33 (i) applies yielding $D_{i, j}(f) \notin \mathfrak{g}_{\ell}^{\prime}$ for some $f \in \mathcal{O}(m)_{\ell+2}$. Since $\mathfrak{g}_{\ell}^{\prime}$ is stable under the normalizer $N_{G_{0}}(T)$ of $T$, which acts doubly transitively on the set of lines $\left\{\mathbb{F} x_{i}^{(1)} \mid 1 \leq\right.$ $i \leq m\}$, we may assume that $(i, j)=(m, 1)$ and $f$ is a weight vector for $T$. Then $f=\xi x_{1}^{\left(a_{1}\right)} x_{2}^{\left(a_{2}\right)} \cdots x_{m}^{\left(a_{m}\right)}$ for some $a_{i} \in \mathbb{N}$ with $\sum_{i=1}^{m} a_{i}=\ell+2$ and $\xi \in \mathbb{F}^{\times}$. Because $\ell+1<p$, the $\mathfrak{s l}_{m-1}$-module generated by $x_{1}^{(\ell+1)} D_{m}$ coincides with $\mathcal{O}(m-1)_{\ell+1} D_{m}$. Consequently, $a_{m} \geq 1$. From (2.31) it follows that $\left[x_{i}^{(1)} D_{j}, D_{m, 1}(g)\right]=D_{m, 1}\left(\left(x_{i}^{(1)} D_{j}\right)(g)\right)$ whenever $2 \leq i, j \leq m-1$. As $\ell+2-a_{1}-a_{m}<p$, the $\mathfrak{s l}_{m-2}$-module $\mathcal{O}(m-2)_{\ell+2-a_{1}-a_{m}}$ is irreducible. Thus, no generality will be lost in assuming that $a_{i}=0$ for $i \notin\{1,2, m\}$. As $\left[x_{2}^{(1)} D_{1}, D_{m, 1}(g)\right]=D_{m, 1}\left(\left(x_{2}^{(1)} D_{1}\right)(g)\right)$ and $a_{1}+a_{2}<p$, we may assume further that $a_{2}=0$. Then $f=x_{1}^{\left(a_{1}\right)} x_{m}^{\left(a_{m}\right)}$ where $a_{1}+a_{m}=\ell+2$.

Let $t$ be the minimal integer with the property that $D_{m, 1}\left(x_{1}^{(t)} x_{m}^{(\ell+2-t)}\right) \notin$ $\mathfrak{g}_{\ell}^{\prime}$. If $t>0$, then $\ell+2-t \not \equiv 0 \bmod p$. In this case Lemma 2.32(c) implies $\left[D_{1, m}\left(x_{m}^{(2)}\right), D_{m, 1}\left(x_{1}^{(t+1)} x_{m}^{(\ell+1-t)}\right)\right]=(\ell+2-t) D_{m, 1}\left(x_{1}^{(t)} x_{m}^{(\ell+2-t)}\right) \in \mathfrak{g}_{\ell}^{\prime} \backslash\{0\}$, a contradiction. Hence $t=0$; that is, $x_{m}^{(\ell+1)} D_{1}=-D_{m, 1}\left(x_{m}^{(\ell+2)}\right) \notin \mathfrak{g}_{\ell}^{\prime}$. On the other hand, $\mathfrak{g}_{\ell}^{\prime}$ is $G_{0}$-stable and there is an element in $N_{G_{0}}(T)$ which permutes the lines spanned by $x_{1}^{(\ell)} D_{m}$ and $x_{m}^{(\ell)} D_{1}$. But then $x_{1}^{(\ell+1)} D_{m} \notin \mathfrak{g}_{\ell}^{\prime}$, which is false. By contradiction, the claim follows. In conjunction with our description of the $\mathfrak{b}^{+}$-primitive vectors in $\mathfrak{g}_{\ell}$, this yields that for $\ell+m \neq 0$ $\bmod p$ the $\mathfrak{g}_{0}^{(1)}$-module $\mathfrak{g}_{\ell}^{\dagger}$ is irreducible, proving (ii).

Now suppose $\ell+m \equiv 0 \bmod p$. Then our earlier remarks show that $\mathfrak{g}_{\ell}^{\sharp}$ coincides with the $\mathfrak{g}_{0}^{(1)}$-socle of $\mathfrak{g}_{\ell}^{\dagger}$. We denote by $M$ the maximal $\mathfrak{g}_{0}^{(1)}$ submodule of $\mathfrak{g}_{\ell}^{\dagger}$. Since all $\mathfrak{g}_{0}^{(1)}$-submodules of $\mathfrak{g}_{\ell}$ are $G_{0}$-stable, so is $M$. Let $\mu$ be a maximal $T$-weight in $X(M)$ and let $v$ be a weight vector of weight $\mu$ in $M$. Then $\left[\mathfrak{n}^{+}, v\right]=0$. Our description of the $\mathfrak{b}^{+}$-primitive vectors in $\mathfrak{g}_{\ell}$ shows that a nonzero multiple of $v$ lies in $\left\{x_{1}^{(\ell+1)} D_{m}, \mathfrak{D}_{x_{1}^{(\ell)}}\right\}$. Since $x_{1}^{(\ell+1)} D_{m}$ generates the $\mathfrak{g}_{0}^{(1)}$-module $\mathfrak{g}_{\ell}^{\dagger}$, it must be that $\mu=\ell \varpi_{1}$, the weight of $\mathfrak{D}_{x_{1}^{(\ell)}}$. Hence, $\nu \leq \ell \varpi_{1}$ for all $\nu \in X(M)$. It also follows that $-\ell \varpi_{m-1}$ is the only minimal weight in $X(M)$ and $\operatorname{dim} M^{\ell \varpi_{1}}=1$.

Next we look at the dual $G_{0}$-module $M^{*}$. Since $X\left(M^{*}\right)=-X(M)$, the preceding remark yields that $\ell \varpi_{m-1}$ is the only maximal weight of $M^{*}$ and $\operatorname{dim}\left(M^{*}\right)^{\ell \varpi_{m-1}}=1$. Let $N:=\left\{\varphi \in M^{*} \mid \varphi(u)=0\right.$ for all $\left.u \in \mathfrak{g}_{\ell}^{\sharp}\right\}$. Clearly, $N$ is a $G_{0}$-submodule of $M^{*}$ and $M^{*} / N \cong\left(\mathfrak{g}_{\ell}^{\sharp}\right)^{*}$. Recall that $\mathfrak{g}_{\ell}^{\sharp} \cong$ $V\left(\ell \varpi_{1}\right) \cong L\left(\ell \varpi_{1}\right)$. Then $M^{*} / N \cong L\left(\ell \varpi_{m-1}\right) \cong V\left(\ell \varpi_{m-1}\right)$; see Proposition 
2.12. Since $\operatorname{dim}\left(M^{*}\right)^{\ell \varpi_{m-1}}=1$, it must be that $\ell \varpi_{m-1} \notin X(N)$. Let $\psi \in\left(M^{*}\right)^{\ell \varpi_{m-1}} \backslash\{0\}$ and denote by $N^{\prime}$ the $G_{0}$-submodule generated by $\psi$. By Proposition 2.14(a), the $G_{0}$-module $N^{\prime}$ is a homomorphic image of the Weyl module $V\left(\ell \varpi_{m-1}\right)$. As the latter is irreducible, so is $N^{\prime}$. On the other hand, $N^{\prime} \not \subset N$ (because $\left.\psi \notin N\right)$. As a consequence, $M^{*}=N \oplus N^{\prime}$ and $M \cong N^{*} \oplus N^{\prime *}$. Since the subspace of $\mathfrak{b}^{+}$-primitive vectors of $M$ is onedimensional, the $G_{0}$-module $M$ is indecomposable. As $N^{\prime} \neq 0$, this forces $N=0$. But then $M=\mathfrak{g}_{\ell}^{\sharp}$, and our proof is complete in the $m \geq 3$ case.

When $m=2$ and $\ell=p-2$, set

$$
v_{k}:=x_{1}^{(p-1-k)} x_{2}^{(k)} D_{2}-x_{1}^{(p-k)} x_{2}^{(k-1)} D_{1} \in \mathfrak{g}_{p-2}^{\dagger}
$$

for $k=0,1, \ldots, p$, where by convention $x_{j}^{(-1)}=0$ for $j=1,2$. The elements $e=x_{1}^{(1)} D_{2}, f=x_{2}^{(1)} D_{1}$, and $h=x_{1}^{(1)} D_{1}-x_{2}^{(1)} D_{2}$ form a canonical basis of $\mathfrak{g}_{0}^{(1)} \cong \mathfrak{s l}_{2}$, and we have

$$
\begin{aligned}
{\left[f, v_{k}\right] } & =(k+1) v_{k+1}, \\
{\left[e, v_{k}\right] } & =(p+1-k) v_{k-1}, \\
{\left[h, v_{k}\right] } & =(p-2 k) v_{k},
\end{aligned}
$$

where $v_{p+1}=0=v_{-1}$. The vectors $v_{k}=k^{-1} x_{1}^{(p-1-k)} x_{2}^{(k-1)} \mathfrak{D}_{1}$ for $k=$ $1, \ldots, p-1$, determine a basis of $\mathfrak{g}_{p-2}^{\sharp}$, and we can see from the expressions in $(2.75)$ that $\mathfrak{g}_{p-2}^{\sharp} \cong L\left((p-2) \varpi_{1}\right)$ as a module for $\mathfrak{g}_{0}^{(1)}$. As $\left[f, v_{0}\right]=v_{1}$ and $\left[e, v_{p}\right]=v_{p-1}$, and the other basis elements of $\mathfrak{g}_{0}^{(1)}$ act trivially on $v_{0}$ and $v_{p}$, we have that $v_{0}$ and $v_{p}$ span trivial $\mathfrak{g}_{0}^{(1)}$-modules of $\mathfrak{g}_{p-2}^{\dagger}$ modulo $\mathfrak{g}_{p-2}^{\sharp}$. Moreover, because $\left[e, x_{1}^{(p-1)} D_{1}\right]=-v_{0}$ and $\left[h, x_{1}^{(p-1)} D_{1}\right]=(p-2) x_{1}^{(p-1)} D_{1}$, the $\mathfrak{g}_{0}^{(1)}$-submodule of $\mathfrak{g}_{p-2} / \mathfrak{g}_{p-2}^{\dagger}$ generated by $x_{1}^{(p-1)} D_{1}$ is isomorphic to $L\left((p-2) \varpi_{1}\right)$. But since $\operatorname{dim} \mathfrak{g}_{p-2}=2 p$, it follows that the $v_{k}(0 \leq k \leq p)$ comprise a basis of $\mathfrak{g}_{p-2}^{\dagger}$ and that $\mathfrak{g}_{p-2} / \mathfrak{g}_{p-2}^{\dagger} \cong L\left((p-2) \varpi_{1}\right)$. Consequently, all the assertions in (v) hold.

The submodules in (2.70) and (2.71) are useful in describing the subalgebras of the restricted Lie algebras of Cartan type which contain $\mathfrak{g}_{-1} \oplus \mathfrak{g}_{0}$.

Proposition 2.76. (Compare [KS, Chap. 1, Sec. 10, Prop.] and [St, Lem. 5.2.3]). Let $\mathfrak{g}=\bigoplus_{j=-1}^{r} \mathfrak{g}_{j}$ be one of the simple restricted Lie algebras $W(m ; \underline{1}), S(m ; \underline{1})^{(1)}$, or $H(2 m ; \underline{1})^{(2)}$, and let $L$ be a (not necessarily graded) subalgebra of $\mathfrak{g}$ containing $\mathfrak{g}_{-1} \oplus \mathfrak{g}_{0}$. Then only the following possibilities can occur:

(a) $L=\mathfrak{g}$,

(b) $L=\mathfrak{g}_{-1} \oplus \mathfrak{g}_{0}$, 
(c) $\mathfrak{g}=W(m ; \underline{1})$ and $L=\mathfrak{g}_{-1} \oplus \mathfrak{g}_{0} \oplus \mathfrak{g}_{1}^{\sharp}$, where $\mathfrak{g}_{1}^{\sharp}$ is as in (2.70), and $L \cong \mathfrak{s l}_{m+1}$ when $m+1 \not \equiv 0 \bmod p$.

(d) $\mathfrak{g}=W(m ; \underline{1})$ and $L=\mathfrak{g}_{-1} \oplus \mathfrak{g}_{0} \oplus \mathfrak{g}_{1}^{\dagger} \oplus L_{2} \oplus \cdots \oplus L_{r-1}$, where $L_{\ell}=\mathfrak{g}_{\ell}^{\dagger}$ for $\ell \neq m(p-1)-p$, and $\left(S(m ; \underline{1})^{(1)}\right)_{\ell} \subseteq L_{\ell} \subseteq \mathfrak{g}_{\ell}^{\dagger}$ for $\ell=m(p-1)-p ;$ thus $S(m ; \underline{1})^{(1)} \oplus \mathbb{F} \mathfrak{D}_{1} \subseteq L \subseteq S(m ; \underline{1}) \oplus \mathbb{F} \mathfrak{D}_{1}$ where $\mathfrak{D}_{1}=\sum_{j=1}^{m} x_{j}^{(1)} D_{j}$;

(e) $\mathfrak{g}=S(m ; \underline{1})^{(1)}$, where $m+1 \equiv 0 \bmod p$, and $L=\mathfrak{g}_{-1} \oplus \mathfrak{g}_{0} \oplus \mathfrak{g}_{1}^{\sharp} \cong$ $\mathfrak{p s l}_{m+1}$.

Proposition 2.77. (See [KS, Chap. 1, Sec. 10] and [St, Lem. 5.2.2].)

(a) The space $H(2 m)_{\ell}$ is irreducible as a module for $H(2 m)_{0}$ whenever $1 \leq \ell \leq p-3$. Moreover, $H(2 m)_{1} \cong \mathcal{O}(2 m)_{3} \cong S^{3}(V)$ where $V$ is the natural $2 m$-dimensional module for $H(2 m)_{0} \cong \mathfrak{s p}_{2 m}$.

(b) The space $H(2 m ; \underline{1})_{\ell}$ is irreducible as a module for $H(2 m)_{0}$ for all $\ell$.

The next lemma is an immediate consequence of Theorem 2.69.

LEMMA 2.78.

(a) Suppose that $\mathfrak{g}=\bigoplus_{j \geq-1} \mathfrak{g}_{j}$ is a Lie algebra of Cartan type $S(m)$ or $C S(m)$ with the natural grading. Relative to the Cartan subalgebra $\mathfrak{h}:=\operatorname{span}_{\mathbb{F}}\left\{D_{\ell, \ell+1}\left(x_{\ell}^{(1)} x_{\ell+1}^{(1)}\right) \mid \ell=1, \ldots, m-1\right\}$ of $\mathfrak{g}_{0}^{(1)}$ and corresponding Borel subalgebras $\mathfrak{b}^{+}$and $\mathfrak{b}^{-}$of $\mathfrak{g}_{0}^{(1)}$, the element $D_{1}$ (resp. $D_{m}$ ) is a $\mathfrak{b}^{-}$-primitive (resp. $\mathfrak{b}^{+}$-primitive) vector of $\mathfrak{g}_{-1}$ of weight $-\varpi_{1}\left(\right.$ resp. $\left.\varpi_{m-1}\right)$. For $k=2,3$, the $\mathfrak{g}_{0}^{(1)}$-module $\mathfrak{g}_{k}$ is generated by the $\mathfrak{b}^{-}$-primitive vector $D_{1, m}\left(x_{m}^{(k+2)}\right)$ of weight $-\varpi_{1}-(k+1) \varpi_{m-1}$.

(b) Suppose that $\mathfrak{g}=\bigoplus_{j \geq-1} \mathfrak{g}_{j}$ is a Lie algebra of Cartan type $H(2 m)$ or $\mathrm{CH}(2 \mathrm{~m})$ with the natural grading. Relative to the Cartan subalgebra $\mathfrak{h}:=\operatorname{span}_{\mathbb{F}}\left\{h_{\ell}=-D_{H}\left(x_{\ell}^{(1)} x_{\ell+m}^{(1)}\right), 1 \leq \ell \leq m\right\}$ of $\mathfrak{g}_{0}^{(1)}$ and corresponding Borel subalgebras $\mathfrak{b}^{+}$and $\mathfrak{b}^{-}$of $\mathfrak{g}_{0}^{(1)}$, the vector $D_{1}=D_{H}\left(x_{1+m}^{(1)}\right)$ is a $\mathfrak{b}^{+}$-primitive vector of $\mathfrak{g}_{-1}$ of weight $\varpi_{1}$. If $p>5$ and $m \geq 2$, the irreducible $\mathfrak{g}_{0}^{(1)}$-module $\mathfrak{g}_{k}$ is generated by the $\mathfrak{b}^{-}$-primitive vector $e^{-(k+2) \varpi_{1}}=D_{H}\left(x_{1+m}^{(k+2)}\right), k=2,3$. If $p=5$, then the $\mathfrak{g}_{0}^{(1)}$-module $\mathfrak{g}_{2}$ is generated by a $\mathfrak{b}^{-}$-primitive vector $e^{-4 \varpi 1}$, and the $\mathfrak{g}_{0}^{(1)}$-module $\mathfrak{g}_{3}$ is generated by a $\mathfrak{b}^{-}$-primitive vector $e^{-3 \varpi_{1}-\varpi_{2}}$ (resp. $\left.e^{-3 \varpi_{1}}\right)$ when $m \geq 2$ (resp. $m=1$ ).

Proof. From Theorem 2.33, we know that $S(m)_{k}$ is spanned by derivations of the form $D_{i, j}(f), f \in \mathcal{O}(m)_{k+2}$ for $k=-1,2,3$. Since by Lemma 2.32(c) and (2.31), 


$$
\begin{aligned}
{\left[D_{1,2}\left(x_{1}^{(1)} x_{2}^{(1)}\right), D_{1, m}\left(x_{m}^{(j)}\right)\right] } & =-D_{1,2}\left(x_{2}^{(1)} x_{m}^{(j-1)}\right) \\
& =-x_{m}^{(j-1)} D_{1}=-D_{1, m}\left(x_{m}^{(j)}\right)
\end{aligned}
$$

and

$$
\begin{aligned}
{\left[D_{m-1, m}\left(x_{m-1}^{(1)} x_{m}^{(1)}\right), D_{1, m}\left(x_{m}^{(j)}\right)\right] } & =-j D_{1, m}\left(x_{m}^{(j)}\right)+D_{1, m-1}\left(x_{m-1}^{(1)} x_{m}^{(j-1)}\right) \\
& =-j x_{m}^{(j-1)} D_{1}+x_{m}^{(j-1)} D_{1} \\
& =-(j-1) D_{1, m}\left(x_{m}^{(j)}\right),
\end{aligned}
$$

and since $\left[D_{\ell, \ell+1}\left(x_{\ell}^{(1)} x_{\ell+1}^{(1)}\right), D_{1, m}\left(x_{m}^{(j)}\right)\right]=0$ for all $\ell \neq 1, m-1$, we see that $D_{1, m}\left(x_{m}^{(j)}\right)$ is a weight vector relative to $\mathfrak{h}$ of weight $-\left(\varpi_{1}+(j-1) \varpi_{m-1}\right)$.

Now it follows from Theorem 2.69 that for $k=2,3$ the $S(m)_{0}$-module $\mathfrak{g}_{k}=S(m)_{k}=\operatorname{span}_{\mathbb{F}}\left\{D \in W(m)_{k} \mid \operatorname{div}(D)=0\right\}$ is generated by a $\mathfrak{b}^{+}$ primitive vector of weight $(k+1) \varpi_{1}+\varpi_{m-1}$ relative to $\mathfrak{h}$. The vector $D_{1, m}\left(x_{m}^{(k+2)}\right)$ is a $\mathfrak{b}^{-}$-primitive vector of $\mathfrak{g}_{k}$ of weight $-\left(\varpi_{1}+(k+1) \varpi_{m-1}\right)$, as required.

We turn our attention now to $\mathfrak{g}$ of Cartan type $H(2 m)$ or $C H(2 m)$. In this case, $\mathfrak{g}_{k}$ is seen to be irreducible for $k \leq p-3$ by [KS, Chap. 1, Sec. 10] (compare also [St, Lem. 5.2.2]). We have the Cartan subalgebra $\mathfrak{h}$ of $\mathfrak{g}_{0}^{(1)}$ spanned by vectors

$$
h_{\ell}=-D_{H}\left(x_{\ell}^{(1)} x_{\ell+m}^{(1)}\right)=x_{\ell}^{(1)} D_{\ell}-x_{\ell+m}^{(1)} D_{\ell+m} \quad(1 \leq \ell \leq m)
$$

(see $(2.50)$ ), and corresponding to the simple roots relative to $\mathfrak{h}$, we have the root vectors

$$
\begin{aligned}
e_{\ell} & =-D_{H}\left(x_{\ell}^{(1)} x_{\ell+1+m}^{(1)}\right)=x_{\ell}^{(1)} D_{\ell+1}-x_{\ell+1+m}^{(1)} D_{\ell+m} & & (1 \leq \ell<m), \\
f_{\ell} & =-D_{H}\left(x_{\ell+1}^{(1)} x_{\ell+m}^{(1)}\right)=x_{\ell+1}^{(1)} D_{\ell}-x_{\ell+m}^{(1)} D_{\ell+1+m} & & (1 \leq \ell<m), \\
e_{m} & =D_{H}\left(x_{m}^{(2)}\right)=x_{m}^{(1)} D_{2 m} & & \\
f_{m} & =-D_{H}\left(x_{2 m}^{(2)}\right)=x_{2 m}^{(1)} D_{m} . & &
\end{aligned}
$$

The $\mathfrak{b}^{-}$-primitive vector of $\mathfrak{g}_{k}$ is realized by $D_{H}\left(x_{1+m}^{(k+2)}\right)$, which has weight $-(k+2) \varpi_{1}$ for $k \leq p-3$. It remains only to show that when $p=5$, the $\mathfrak{g}_{0}^{(1)}$-module $\mathfrak{g}_{3}$ is generated by a $\mathfrak{b}^{-}$-primitive vector $e^{-\left(3 \varpi_{1}+\varpi_{2}\right)}$ of weight $-3 \varpi_{1}-\varpi_{2}$. But that follows from Lemma 2.79 below.

Lemma 2.79. Let $V$ be the natural $2 m$-dimensional module for $\mathfrak{s p}_{2 m}$. If $p>5$, then $S^{5}(V)$ is an irreducible $\mathfrak{s p}_{2 m}$-module. If $p=5$, then $S^{5}(V)$ has a trivial submodule $Y:=\operatorname{span}_{\mathbb{F}}\left\{\left(x_{i}^{(1)}\right)^{5} \mid i=1, \ldots, 2 m\right\}$. The quotient module $S^{5}(V) / Y$ is an irreducible module for $\mathfrak{s p}_{2 m}$ with a $\mathfrak{b}^{+}$-primitive vector of weight $3 \varpi_{1}+\varpi_{2}\left(3 \varpi_{1}\right.$ if $\left.m=1\right)$. 
Proof. We identify $V$ with $\mathcal{O}(2 m ; \underline{1})_{1}$ and the action of $\mathfrak{s p}_{2 m}$ with that of $H(2 m)_{0}$ on $\mathcal{O}(2 m ; \underline{1})_{1}$. Then when $p>5$, we can identify $S^{5}(V)$ with $\mathcal{O}(2 m ; \underline{1})_{5}$. By [St, Lem. 5.2.2], the $H(2 m)_{0}$-module $\mathcal{O}(2 m ; \underline{1})_{k}$ is irreducible for all $k$. Thus, the result holds for $p>5$. Now when $p=5$, the space $Y:=\operatorname{span}_{\mathbb{F}}\left\{\left(x_{i}^{(1)}\right)^{5} \mid i=1, \ldots, 2 m\right\}$ is a trivial $\mathfrak{s p}_{2 m}$-submodule of $S^{5}(V)$. The image of $\left(x_{1}^{(1)}\right)^{4} x_{2}^{(1)}$ in $S^{5}(V) / Y$ is a $\mathfrak{b}^{+}$-primitive vector of weight $3 \varpi_{1}+\varpi_{2}$ if $m \geq 2\left(3 \varpi_{1}\right.$ if $\left.m=1\right)$ relative to the Cartan subalgebra $\mathfrak{h}$ in the proof of Lemma 2.78(b). Since $\operatorname{dim} S^{5}(V) / Y=\operatorname{dim} H(2 m ; \underline{1})_{3}$, and $H(2 m ; \underline{1})_{3}$ is an irreducible module for $\mathfrak{s p}_{2 m} \cong H(2 m)_{0}$ by Proposition 2.77(b), it follows from Lemma 2.78(b) that $S^{5}(V) / Y$ is a irreducible $\mathfrak{s p}_{2 m^{-}}$ module with a $\mathfrak{b}^{+}$-primitive vector of weight $3 \varpi_{1}+\varpi_{2}$ if $m \geq 2\left(3 \varpi_{1}\right.$ if $m=1)$.

Lemma 2.80. Assume $p=5$. Then

$H(2 ; \underline{1})^{(1)}=\tilde{H}(2 ; \underline{1})=\left\{D_{H}(f) \mid f \in \mathcal{O}(2 ; \underline{1})\right\}=H(2 ; \underline{1})^{(2)} \oplus \mathbb{F} D_{H}\left(x_{1}^{(4)} x_{2}^{(4)}\right)$,

where $D_{H}\left(x_{1}^{(4)} x_{2}^{(4)}\right) \in\left(H(2 ; \underline{1})^{(1)}\right)_{6}$. If $\mathfrak{g}=\bigoplus_{i=-1}^{r} \mathfrak{g}_{i}$ is a restricted Lie algebra of Cartan type $H(2 ; \underline{1})$ or $C H(2 ; \underline{1})$, and $\widehat{\mathfrak{g}}$ is the subalgebra of $\mathfrak{g}$ generated by the local part $\mathfrak{g}_{-1} \oplus \mathfrak{g}_{0} \oplus \mathfrak{g}_{1}$, then

(a) $\widehat{\mathfrak{g}}_{3}=\mathfrak{g}_{3}=\operatorname{span}_{\mathbb{F}}\left\{D_{H}(f) \mid f=x_{1}^{(4)} x_{2}^{(1)}, x_{1}^{(3)} x_{2}^{(2)}, x_{1}^{(2)} x_{2}^{(3)}, x_{1}^{(1)} x_{2}^{(4)}\right\}$ is an irreducible $\mathfrak{g}_{0}^{(1)}$-module generated by the $\mathfrak{b}^{-}$-primitive vector $D_{H}\left(x_{1}^{(1)} x_{2}^{(4)}\right)$ of weight $-3 \varpi_{1}$;

(b) $(\widehat{\mathfrak{g}})^{(1)}=H(2 ; 1)^{(2)}$ is a simple Lie algebra;

(c) $\widehat{\mathfrak{g}}_{i} \subset(\widehat{\mathfrak{g}})^{(1)}$ for all $i \neq 0$;

(d) $\widehat{\mathfrak{g}}_{6}=0$.

Proof. This result is apparent from Theorem 2.38 (iii) and equations (2.46) and (2.47).

\subsection{Melikyan Lie algebras}

In characteristic 5, there exist finite-dimensional simple Lie algebras which are neither classical nor of Cartan type. Here we quote from [St, Sec. 4.3], which in turn follows the presentation of [Ku2].

Let $\widetilde{W}(2 ; \underline{n})$ denote a second copy of the vector space $W(2 ; \underline{n})$ and set

$$
M(2 ; \underline{n}):=\mathcal{O}(2 ; \underline{n}) \oplus W(2 ; \underline{n}) \oplus \widetilde{W}(2 ; \underline{n})
$$


The space $M(2 ; \underline{n})$ can be given the structure of a Lie algebra by defining an anticommutative bracket multiplication as follows:

$$
\begin{gathered}
{[D, \widetilde{E}]=\widetilde{[D, E]}+2 \operatorname{div}(D) \widetilde{E}} \\
{[D, f]=D(f)-2 \operatorname{div}(D) f} \\
{\left[f_{1} \widetilde{D_{1}}+f_{2} \widetilde{D_{2}}, g_{1} \widetilde{D_{1}}+g_{2} \widetilde{D_{2}}\right]=f_{1} g_{2}-f_{2} g_{1}} \\
{[f, \widetilde{E}]=f E} \\
{[f, g]=2\left(f \widetilde{D}_{g}-g \widetilde{D}_{f}\right) \quad \text { where }} \\
\widetilde{D}_{f}=D_{1}(f) \widetilde{D}_{2}-D_{2}(f) \widetilde{D_{1}} .
\end{gathered}
$$

for all $D \in W(2 ; \underline{n}), \widetilde{E} \in \widetilde{W}(2 ; \underline{n})$, and $f, g, f_{i}, g_{i}(i=1,2)$ in $\mathcal{O}(2 ; \underline{n})$. The product $[D, E]$ of two elements of $W(2 ; \underline{n}) \subset M(2 ; \underline{n})$ is the same as in the Witt Lie algebra of Cartan type $W(2 ; \underline{n})$.

Although the above makes sense for any prime $p$, it is only for $p=5$ that the multiplication defined by (2.82) satisfies the Jacobi identity; the proof of this fact can be found in [St, Lem. 4.3.1]. The Lie algebras $M(2 ; \underline{n})$ are called the Melikyan algebras. From the construction we see that

$$
\operatorname{dim} M(2 ; \underline{n})=5^{n_{1}+n_{2}}+2 \cdot 5^{n_{1}+n_{2}}+2 \cdot 5^{n_{1}+n_{2}}=5^{n_{1}+n_{2}+1} .
$$

The subspace $W(2 ; \underline{n})$ of $M(2 ; \underline{n})$ is a Lie subalgebra of $M(2 ; \underline{n})$, and both $\mathcal{O}(2 ; \underline{n})$ and $\widetilde{W}(2 ; \underline{n})$ are $W(2 ; \underline{n})$-modules.

We assign gradation degrees $\operatorname{deg}_{M}(\cdot)$ to elements of $M(2 ; \underline{n})$, by using the natural gradation degrees $\operatorname{deg}_{W}$ in the Lie algebra $W(2 ; \underline{n})$ and the degrees $\operatorname{deg}_{\mathcal{O}}$ in the associative algebra $\mathcal{O}(2 ; \underline{n})$ :

$$
\begin{aligned}
\operatorname{deg}_{M}(D) & =3 \operatorname{deg}_{W}(D) \\
\operatorname{deg}_{M}(\widetilde{E}) & =3 \operatorname{deg}_{W}(E)+2 \\
\operatorname{deg}_{M}(f) & =3 \operatorname{deg}_{\mathcal{O}}(f)-2,
\end{aligned}
$$

where $D, \widetilde{E}$, and $f$ are as above. Thus, $M(2 ; \underline{n})=\bigoplus_{j=-3}^{r} M(2 ; \underline{n})_{j}$, where $r=3\left(5^{n_{1}}+5^{n_{2}}\right)-7$. We refer to this as the natural grading of $M(2 ; \underline{n})$, a phrase motivated by the fact that $\bigoplus_{j>0}^{r} M(2 ; \underline{n})_{j}$ is the unique maximal subalgebra of codimension 5 and depth $\geq 3$ (see [St, Thm. 4.3.3], which is based on $[\mathbf{K u 2}])$. The natural grading determines a $\mathbb{Z} / 3 \mathbb{Z}$-grading $M(2 ; \underline{n})=$ $M_{\overline{-2}} \oplus M_{\overline{0}} \oplus M_{\overline{2}}$ where $M_{\overline{-2}}=\mathcal{O}(2 ; \underline{n}), M_{\overline{0}}=W(2 ; \underline{n})$, and $M_{\overline{2}}=\widetilde{W}(2 ; \underline{n})$.

The natural gradation on $M(2 ; \underline{n})$ is inspired by a certain gradation of a classical simple Lie algebra $\mathcal{G}$ of type $\mathrm{G}_{2}$. Suppose $\mathcal{H}$ is a Cartan subalgebra of $\mathcal{G}$ and $\{\alpha, \beta\}$ is a base of simple roots with $\alpha$ short and $\beta$ long. We assign $\alpha$ degree $1,-\alpha$ degree -1 , and $\pm \beta$ degree 0 . Then $\mathcal{G}=\bigoplus_{j=-3}^{3} \mathcal{G}_{j}$, where 


$$
\begin{gathered}
\mathcal{G}_{-3}=\mathcal{G}^{-3 \alpha-2 \beta} \oplus \mathcal{G}^{-3 \alpha-\beta}, \quad \mathcal{G}_{-2}=\mathcal{G}^{-2 \alpha-\beta}, \quad \mathcal{G}_{-1}=\mathcal{G}^{-\alpha-\beta} \oplus \mathcal{G}^{-\alpha} \\
\mathcal{G}_{0}=\mathcal{G}^{-\beta} \oplus \mathcal{H} \oplus \mathcal{G}^{\beta} \\
\mathcal{G}_{1}=\mathcal{G}^{\alpha+\beta} \oplus \mathcal{G}^{\alpha}, \quad \mathcal{G}_{2}=\mathcal{G}^{2 \alpha+\beta}, \quad \mathcal{G}_{3}=\mathcal{G}^{3 \alpha+2 \beta} \oplus \mathcal{G}^{3 \alpha+\beta} .
\end{gathered}
$$

Indeed for the Melikyan algebra $M=M(2 ; \underline{n})$, we have

$$
\begin{gathered}
M_{-3}=\mathbb{F} D_{1} \oplus \mathbb{F} D_{2}, \quad M_{-2}=\mathbb{F} 1, \quad M_{-1}=\mathbb{F} \widetilde{D_{1}} \oplus \mathbb{F} \widetilde{D_{2}} \\
M_{0}=\operatorname{span}_{\mathbb{F}}\left\{x_{i}^{(1)} D_{j} \mid i, j=1,2\right\} \\
M_{1}=\mathbb{F} x_{1}^{(1)} \oplus \mathbb{F} x_{2}^{(1)},
\end{gathered}
$$

so that $\bigoplus_{j \leq 0} M_{j} \cong \bigoplus_{j \leq 0} \mathcal{G}_{j}$ as graded Lie algebras.

The Melikyan algebras can be characterized by their gradation.

Proposition 2.84. (See [Ku2] and [St, Thm. 5.4.1].) Let $\mathfrak{g}=\bigoplus_{j=-3}^{r} \mathfrak{g}_{j}$ be a finite-dimensional, transitive and 1-transitive graded Lie algebra of height $r$, and suppose that $\bigoplus_{j=-3}^{0} \mathfrak{g}_{j} \cong \bigoplus_{j=-3}^{0} M(2 ; \underline{1})_{j}$ as graded Lie algebras. If $r \leq 3$, then $\mathfrak{g}$ is isomorphic to a classical Lie algebra of type $\mathrm{G}_{2}$. If $r>3$, then $\mathfrak{g}$ is isomorphic as a graded Lie algebra to a Melikyan algebra $M(2 ; \underline{n})$ with its natural grading.

Proof. By our assumption, $\mathfrak{g}_{0} \cong \mathfrak{g l}_{2}$, both $\mathfrak{g}_{-1}$ and $\mathfrak{g}_{-3}$ are two-dimensional, irreducible faithful $\mathfrak{g}_{0}$-modules, and $\mathfrak{g}_{-}$is generated by $\mathfrak{g}_{-1}$. Since $\mathfrak{g}$ is $1-$ transitive, Proposition 1.13 shows that $\mathfrak{g}$ contains a unique minimal ideal $\mathfrak{J}$ which is graded and contains $\mathfrak{g}_{-1}$.

Since $\operatorname{dim} \mathfrak{g}_{-2}=1$, the Lie product on $\mathfrak{g}$ induces a $\mathfrak{g}_{0}^{(1)}$-equivariant pairing $\mathfrak{g}_{-3} \times \mathfrak{g}_{1} \rightarrow \mathbb{F}$. The 1-transitivity of $\mathfrak{g}$ along with the above remark implies that this pairing is nondegenerate. As a result, $\mathfrak{g}_{1} \cong \mathfrak{g}_{-3} \cong \mathfrak{g}_{-1}$ as $\mathfrak{g}_{0}^{(1)}$-modules.

The Lie product on $\mathfrak{g}$ induces a $\mathfrak{g}_{0}$-module isomorphism $\mathfrak{g}_{-3} \cong \mathfrak{g}_{-2} \otimes$ $\mathfrak{g}_{-1}$. Choose $u_{1} \in \mathfrak{g}_{1}$ and $u_{-3}, v_{-3} \in \mathfrak{g}_{-3} \backslash\{0\}$ such that $\left[u_{1}, u_{-3}\right] \neq 0$ and $\left[u_{1}, v_{-3}\right]=0$. Choose $u_{-1}, v_{-1} \in \mathfrak{g}_{-1}$ such that $\left[u_{-1},\left[u_{1}, u_{-3}\right]\right]=u_{-3}$ and $\left[v_{-1},\left[u_{1}, u_{-3}\right]\right]=v_{-3}$. Then $\left[\left[u_{1}, u_{-1}\right], u_{-3}\right]=\left[\left[u_{1}, u_{-3}\right], u_{-1}\right]=-u_{-3}$ and $\left[\left[u_{1}, u_{-1}\right], v_{-3}\right]=0$. Hence, $\left[\mathfrak{g}_{-1}, \mathfrak{g}_{1}\right]$ is a noncentral ideal of $\mathfrak{g}_{0}$, and $\left[\mathfrak{g}_{-1}, \mathfrak{g}_{1}\right] \nsubseteq \mathfrak{g}_{0}^{(1)}$. But then $\mathfrak{g}_{0}=\left[\mathfrak{g}_{-1}, \mathfrak{g}_{1}\right]$ implying $\mathcal{J}_{0}=\mathfrak{g}_{0}$ and $\mathcal{J}_{1}=\mathfrak{g}_{1}$.

By Lemma 1.16, we have $\mathcal{J}=\bigoplus_{j=-3}^{s} \mathcal{J}_{j}$ where $s \in\{r-1, r\}$. Clearly, $\left[\mathfrak{g}_{i}, \mathcal{J}_{s}\right]=0$ for all $i>0$. Since any $\mathfrak{g}_{0}$-submodule $\mathcal{K}_{s}$ of $\mathcal{J}_{s}$ generates an ideal $\bigoplus_{i \geq 0}\left(\operatorname{ad} \mathfrak{g}_{-1}\right)^{i}\left(\mathcal{K}_{s}\right)$ of $\mathfrak{g}$ contained in $\mathcal{J}$, the minimality of $\mathcal{J}$ shows that $\mathcal{J}_{s}$ is an irreducible $\mathfrak{g}_{0}$-module. If $\left[\mathfrak{g}_{0}, \mathcal{J}_{s}\right]=0$, then $\bigoplus_{i \geq 1}\left(\operatorname{ad} \mathfrak{g}_{-1}\right)^{i}\left(\mathcal{J}_{s}\right)$ is an ideal of $\mathfrak{g}$ contained in $\bigoplus_{j=-3}^{s-1} \mathcal{J}_{j}$, a contradiction. Therefore, $\mathcal{J}_{s}=\left[\mathfrak{g}_{0}, \mathcal{J}_{s}\right]$.

Let $\mathcal{J}$ be an arbitrary nonzero ideal of the Lie algebra $\mathcal{J}$. Then $\mathfrak{g}_{-3} \subset \mathcal{J}$ in view of transitivity and the fact that $\mathfrak{g}_{-1} \subset \mathcal{J}$. Since $\mathfrak{g}_{1} \subset \mathcal{J}$ and $\mathfrak{g}$ is 
1-transitive, it follows that $\mathfrak{g}_{-1} \subset \mathcal{J}$. But then $\mathfrak{g}_{0}=\left[\mathfrak{g}_{-1}, \mathfrak{g}_{1}\right] \subset \mathcal{J}$ yielding $\mathcal{J}_{s}=\left[\mathfrak{g}_{0}, \mathcal{J}_{s}\right] \subset \mathcal{J}$. This implies $\mathcal{J}=\bigoplus_{i \geq 0}\left(\operatorname{ad} \mathfrak{g}_{-1}\right)^{i}\left(\mathcal{J}_{s}\right) \subseteq \mathcal{J}$. We conclude that $\mathcal{J}$ is a simple Lie algebra.

For $k \geq-3$, set $\mathcal{J}_{(k)}:=\bigoplus_{j \geq k} \mathcal{J}_{j}$. Clearly, $\left[\mathcal{J}_{(i)}, \mathcal{J}_{(j)}\right] \subseteq \mathcal{J}_{(i+j)}$ for all $i, j \in \mathbb{Z}$, that is $\mathcal{J}=\mathcal{J}_{(-3)} \supset \cdots \supset \mathcal{J}_{(s)} \supset 0$ is a filtration of the Lie subalgebra $\mathcal{J}$. By construction, the corresponding graded Lie algebra $\bigoplus_{j \geq 3}\left(\mathcal{J}_{(j)} / \mathcal{J}_{(j+1)}\right)$ is isomorphic to the graded Lie algebra $\mathcal{J}$. It is straightforward to check that $\mathcal{J}_{(0)}$ is a maximal subalgebra of $\mathcal{J}$. Since $\mathcal{J}$ is a simple Lie algebra we now can apply [St, Thm. 5.4.1] to deduce that either $s=3$ and $\mathcal{J}$ is isomorphic as a graded algebra to a simple Lie algebra of type $\mathrm{G}_{2}$, or $s>3$ and $\mathcal{J}$ is isomorphic as a graded algebra to a Melikyan algebra $M(2, \underline{n})$ with the natural grading.

The adjoint action of $\mathfrak{g}$ on $\mathcal{J}$ gives rise to a Lie algebra homomorphism $\pi: \mathfrak{g} \rightarrow \operatorname{Der}(\mathcal{J})$. If ker $\pi \neq 0$, then ker $\pi \supseteq \mathcal{J}$, by the minimality of $\mathcal{J}$. Since $[\mathcal{J}, \mathcal{J}] \neq 0$, this is impossible. So $\pi$ is injective. If $\mathcal{J}$ is a Lie algebra of type $\mathrm{G}_{2}$, then all derivations of $\mathcal{J}$ are inner, forcing $\mathfrak{g}=\mathcal{J}$. Now suppose $\mathcal{J} \cong M(2 ; \underline{n})$, and identify $\mathfrak{g}$ with a subalgebra of $\operatorname{Der}(M(2 ; \underline{n}))$. Note that the natural gradation of $M(2 ; \underline{n})$ gives rise to a grading of the derivation algebra $\operatorname{Der}(M(2 ; \underline{n}))$ relative to which $\pi: \mathfrak{g} \rightarrow \operatorname{Der}(M(2 ; \underline{n}))$ becomes a homomorphism of graded Lie algebras. According to [St, Thm. 7.1.4], the Lie algebra $\operatorname{Der}(M(2 ; \underline{n}))$ is spanned by ad $M(2 ; \underline{n})$ and the $p$ th powers of $D_{1}, D_{2} \in W(2 ; \underline{n})=M(2 ; \underline{n})_{\overline{0}}$. Since $\operatorname{deg}\left(D_{i}\right)^{p^{j}}=-3 p^{j}$ for all $j \geq 0$, this description implies that

$$
\pi(\mathfrak{g}) \subseteq \bigoplus_{j \geq-3}(\operatorname{Der}(M(2 ; \underline{n})))_{j}=\operatorname{ad} M(2 ; \underline{n}) .
$$

Thus, $\mathfrak{g}=\mathcal{J} \cong M(2 ; \underline{n})$, completing the proof. 


\section{CHAPTER 3}

\section{The Contragredient Case}

\subsection{Introduction}

This chapter is devoted to a proof of the Main Theorem for graded Lie algebras $\mathfrak{g}=\bigoplus_{j=-q}^{r} \mathfrak{g}_{j}$ in which $\mathfrak{g}_{-1}$ and $\mathfrak{g}_{1}$ are dual modules for $\mathfrak{g}_{0}$ (the so-called contragredient case). We begin by focusing on three-dimensional subalgebras of $\mathfrak{g}$ and their representations. We investigate conditions under which certain pairs of such subalgebras, which share a common onedimensional Cartan subalgebra, generate an infinite-dimensional algebra. Next we focus on extreme vectors in $\mathfrak{g}_{1}$ and $\mathfrak{g}_{-1}$ relative to Borel subalgebras $\mathfrak{b}^{+}$and $\mathfrak{b}^{-}$of $\mathfrak{g}_{0}$ and show that a $\mathfrak{b}^{+}$-primitive vector of $\mathfrak{g}_{1}$ and a $\mathfrak{b}^{-}$-primitive vector of $\mathfrak{g}_{-1}$ generate a three-dimensional simple Lie algebra. We then proceed to show that $\mathfrak{g}$ contains a subalgebra with a "balanced" gradation and use that fact to conclude that either $\mathfrak{g}$ is a classical Lie algebra or the characteristic of $\mathbb{F}$ is 5 and $\mathfrak{g}$ is isomorphic to a Melikyan Lie algebra.

\subsection{Results on modules for three-dimensional Lie algebras}

In this section, we create computational tools for dealing with modules for three-dimensional simple Lie algebras and Heisenberg Lie algebras and apply them to show that certain pairs of such Lie algebras sharing a common Cartan subalgebra generate an infinite-dimensional Lie algebra.

The first result, which can be proved easily by induction, concerns modules for a Lie algebra $\mathfrak{s}$ spanned by elements $e, f, h$ satisfying the commutation relations

$$
[e, f]=h, \quad[h, e]=\xi e, \quad[h, f]=-\xi f .
$$

When $\xi$ is specialized to be 2 (or equivalently, any nonzero scalar as $p>$ 2 ), the result gives information about $\mathfrak{s l}_{2}$-modules; when $\xi=0$, the result applies to modules for a Heisenberg Lie algebra.

Proposition 3.2. $\quad$ (a) Let $\mathcal{U}$ be a module for $\mathfrak{s}=\operatorname{span}_{\mathbb{F}}\{e, f, h\}$, where $e, f, h$ satisfy the commutation relations in (3.1), and suppose $\mathcal{U}$ contains a vector $u_{0} \neq 0$ such that

$$
h . u_{0}=\mu u_{0} \quad f . u_{0}=0 .
$$


Set $u_{-1}=0$ and $u_{j}=e \cdot u_{j-1}$ for $j=1,2, \ldots$. Then

$$
f \cdot u_{j}=-\left(j \mu+\frac{j(j-1)}{2} \xi\right) u_{j-1}
$$

for all $j=0,1, \ldots$ If $f . u_{j}=0$ for some $j$, then $j \equiv 0 \bmod p$ or $2 \mu=-(j-1) \xi$.

(b) Let $\mathcal{V}$ be a module for $\mathfrak{s}=\operatorname{span}_{\mathbb{F}}\{e, f, h\}$, and suppose $\mathcal{V}$ contains a vector $v_{0} \neq 0$ such that

$$
h . v_{0}=\lambda v_{0} \quad e . v_{0}=0 .
$$

Set $v_{-1}=0$ and $v_{j}=f . v_{j-1}$ for $j=1,2, \ldots$. Then

$$
e . v_{j}=\left(j \lambda-\frac{j(j-1)}{2} \xi\right) v_{j-1}
$$

for all $j=0,1, \ldots$ If e. $v_{j}=0$ for some $j$, then $j \equiv 0 \bmod p$ or $2 \lambda=(j-1) \xi$.

In the next result and subsequent ones, we will adopt the convention of using the corresponding capital letter for the adjoint mapping. Thus, $E=\operatorname{ad} e$, etc.

Proposition 3.3. Suppose $\mathfrak{s}=\operatorname{span}_{\mathbb{F}}\{e, f, h\}$ is a subalgebra inside a Lie algebra $\mathfrak{g}$, and the elements $e, f, h$ satisfy the commutation relations in (3.1). Assume $u_{0}, v_{0}$ are elements of $\mathfrak{g}$ such that

$$
\begin{array}{cr}
F u_{0}=0 & H u_{0}=-\lambda u_{0}, \\
E v_{0}=0 & H v_{0}=\lambda v_{0}, \\
& {\left[u_{0}, v_{0}\right]=h .}
\end{array}
$$

Then for $\ell \in\{2, \ldots, p-1\},\left[E^{\ell} u_{0}, F^{\ell} v_{0}\right]$ is a nonzero multiple of $h$ if and only if $2 \lambda \neq(k-1) \xi$ for $k=2, \ldots, \ell$ and $\lambda \neq \ell \xi$.

Proof. Observe that

$$
\left.\left.\operatorname{ad}\left(E^{\ell} u_{0}\right)=[\underbrace{E, \ldots[E,[E}_{\ell}, U_{0}]\right] \ldots\right]=\sum_{k=0}^{\ell}(-1)^{\ell}\left(\begin{array}{l}
\ell \\
k
\end{array}\right) E^{\ell-k} U_{0} E^{k} .
$$

Thus

$$
\left[E^{\ell} u_{0}, F^{\ell} v_{0}\right]=\sum_{k=0}^{\ell}(-1)^{k}\left(\begin{array}{l}
\ell \\
k
\end{array}\right) E^{\ell-k} U_{0} E^{k} F^{\ell} v_{0} .
$$

Now by Proposition $3.2(\mathrm{~b}), E^{k}$ acting on $F^{\ell} v_{0}$ is a multiple of $F^{\ell-k} v_{0}$, and $U_{0} F^{\ell-k} v_{0}=F^{\ell-k} U_{0} v_{0}=F^{\ell-k} h$, which is 0 if $\ell-k \geq 2$. Thus, 


$$
\begin{aligned}
{\left[E^{\ell} u_{0}, F^{\ell} v_{0}\right]=} & (-1)^{\ell-1} \ell E U_{0} E^{\ell-1} F^{\ell} v_{0}+(-1)^{\ell} U_{0} E^{\ell} F^{\ell} v_{0} \\
= & (-1)^{\ell-1} \ell \prod_{k=2}^{\ell}\left(k \lambda-\frac{k(k-1)}{2} \xi\right) E U_{0} F v_{0} \\
& \quad+(-1)^{\ell} \prod_{k=1}^{\ell}\left(k \lambda-\frac{k(k-1)}{2} \xi\right) U_{0} v_{0} \\
& (-1)^{\ell-1} \prod_{k=2}^{\ell}\left(k \lambda-\frac{k(k-1)}{2} \xi\right)(\ell \xi-\lambda) h .
\end{aligned}
$$

We obtain a nonzero multiple of $h$ precisely when $2 \lambda \neq(k-1) \xi$ for any $k=2, \ldots, \ell$ and $\lambda \neq \ell \xi$.

Proposition 3.6. Suppose $\mathfrak{g}$ is a Lie algebra containing nonzero elements $e_{i}, f_{i}, i=1,2$, and $h$ such that

$$
\begin{array}{cc}
{\left[h, e_{1}\right]=2 e_{1},} & {\left[h, f_{1}\right]=-2 f_{1},} \\
{\left[h, e_{2}\right]=a e_{2},} & {\left[h, f_{2}\right]=-a f_{2},} \\
{\left[e_{i}, f_{j}\right]=\delta_{i, j} h,}
\end{array}
$$

for some $a \neq 0$. If $a \in\{1,2, \ldots, p-1\}=\mathbb{F}_{p} \backslash\{0\}$, set

$$
\begin{aligned}
e_{1}^{\prime}=F_{1}^{p-a} f_{2}, & f_{1}^{\prime}=E_{1}^{p-a} e_{2}, \\
e_{2}^{\prime}=F_{2}^{2} e_{1}^{\prime}=F_{2}^{2} F_{1}^{p-a} f_{2}, & f_{2}^{\prime}=E_{2}^{2} f_{1}^{\prime}=E_{2}^{2} E_{1}^{p-a} e_{2} .
\end{aligned}
$$

If $a \notin \mathbb{F}_{p}$, set

$$
\begin{aligned}
e_{1}^{\prime}=E_{1}^{p-2} e_{2}, & f_{1}^{\prime}=F_{1}^{p-2} f_{2}, \\
e_{2}^{\prime}=E_{2}^{p-2} E_{1}^{2} e_{2}, & f_{2}^{\prime}=F_{2}^{p-2} F_{1}^{2} f_{2} .
\end{aligned}
$$

Then we have

$$
\begin{array}{rrr}
{\left[h, e_{1}^{\prime}\right]=b e_{1}^{\prime},} & {\left[h, f_{1}^{\prime}\right]=-b f_{1},} \\
{\left[h, e_{2}^{\prime}\right]=-b e_{2}^{\prime},} & {\left[h, f_{2}^{\prime}\right]=b f_{2}^{\prime},} \\
{\left[e_{i}, f_{j}\right]=\delta_{i, j} \zeta_{i} h,}
\end{array}
$$

where $\zeta_{1}$ and $\zeta_{2}$ are nonzero scalars, and $b=a$ for $a \in \mathbb{F}_{p}$ and $b=a-4$ for $a \notin \mathbb{F}_{p}$.

Proof. First we assume that $a \in\{1,2, \ldots, p-1\}$. Suppose $a \neq p-1$. Then $\left[e_{2}, e_{1}^{\prime}\right]=0=\left[f_{2}, f_{1}^{\prime}\right]$, from which it follows that 


$$
\begin{aligned}
& {\left[e_{1}^{\prime}, f_{2}^{\prime}\right]=\left[e_{1}^{\prime}, E_{2}^{2} f_{1}^{\prime}\right] \in \mathbb{F} E_{2}^{2} h=0} \\
& {\left[e_{2}^{\prime}, f_{1}^{\prime}\right]=\left[F_{2}^{2} e_{1}^{\prime}, f_{1}^{\prime}\right] \in \mathbb{F} F_{2}^{2} h=0 .}
\end{aligned}
$$

Applying Proposition 3.3 with $\mathfrak{s}=\operatorname{span}_{\mathbb{F}}\left\{e_{1}, f_{1}, h\right\}, u_{0}=e_{2}, v_{0}=f_{2}, \lambda=$ $p-a, \xi=2$, and $\ell=p-a$ shows that $\left[e_{1}^{\prime}, f_{1}^{\prime}\right]=-\left[E_{1}^{p-a} e_{2}, F_{1}^{p-a} f_{2}\right]$ is a nonzero multiple of $h$, say $\mu h$.

Next take $\mathfrak{s}=\operatorname{span}_{\mathbb{F}}\left\{e_{2}, f_{2}, h\right\}, u_{0}=f_{1}^{\prime}$, and $v_{0}=-\mu^{-1} e_{1}^{\prime}$. Notice that $\lambda=\xi=a$ in this case. Applying Proposition 3.3 with $\ell=2$, we see that $\left[E_{2}^{2} u_{0}, F_{2}^{2} v_{0}\right]=-\mu^{-1}\left[e_{2}^{\prime}, f_{2}^{\prime}\right]$ is a nonzero multiple of $h$. This finishes the proof of the proposition for $a \in\{1,2, \ldots, p-2\}$.

Suppose $a=p-1$. Then $e_{1}^{\prime}=F_{1} f_{2}, f_{1}^{\prime}=E_{1} e_{2}, e_{2}^{\prime}=F_{2}^{2} e_{1}^{\prime}=-F_{2}^{3} f_{1}$ and $f_{2}^{\prime}=E_{2}^{2} f_{1}^{\prime}=-E_{2}^{3} e_{1}$. Direct calculation shows that

$$
\begin{gathered}
{\left[e_{1}^{\prime}, f_{2}^{\prime}\right]=0=\left[e_{2}^{\prime}, f_{1}^{\prime}\right]} \\
{\left[e_{1}^{\prime}, f_{1}^{\prime}\right]=-h .}
\end{gathered}
$$

Using Proposition 3.3 once again with $\mathfrak{s}=\operatorname{span}_{\mathbb{F}}\left\{e_{2}, f_{2}, h\right\}, u_{0}=e_{1}, v_{0}=f_{1}$, $\lambda=p-2, \xi=p-1$, and $\ell=3$ allows us to conclude that $\left[e_{2}^{\prime}, f_{2}^{\prime}\right]$ is a nonzero multiple of $h$ also.

Now assume that $a \notin \mathbb{F}_{p}$ and take $\mathfrak{s}=\operatorname{span}_{\mathbb{F}}\left\{e_{1}, f_{1}, h\right\}, u_{0}=e_{2}$, and $v_{0}=f_{2}$. Then $\lambda=-a$ and $\xi=2$. Applying Proposition 3.3 with $\ell=p-2$ shows that $\left[e_{1}^{\prime}, f_{1}^{\prime}\right]=\left[E_{1}^{p-2} u_{0}, F_{1}^{p-2} v_{0}\right]$ is a nonzero multiple of $h$, say $\nu h$.

Finally, take $\mathfrak{s}=\operatorname{span}_{\mathbb{F}}\left\{e_{2}, f_{2}, h\right\}, u_{0}=E_{1}^{2} e_{2}, v_{0}=\nu^{-1} F_{1}^{2} f_{2}$, and $\ell=$ $p-2$ in Proposition 3.3, where $\nu=2(a+1)(a+4)$. Since $\lambda=-(a+4)$ and $\xi=a$ in this case, we see that $\left[E_{2}^{p-2} u_{0}, F_{2}^{p-2} v_{0}\right]=\nu^{-1}\left[e_{2}^{\prime}, f_{2}^{\prime}\right]$ is a nonzero multiple of $h$. Moreover, because $\left[e_{1}^{\prime}, f_{2}\right]=0=\left[f_{1}^{\prime}, e_{2}\right]$ and $p-2 \geq 3$, we have

$$
\begin{aligned}
& {\left[e_{1}^{\prime}, f_{2}^{\prime}\right]=\left[e_{1}^{\prime}, F_{2}^{p-2} F_{1}^{2} f_{2}\right]=F_{2}^{p-2}\left[E_{1}^{p-2} e_{2}, F_{1}^{2} f_{2}\right]=0} \\
& {\left[e_{2}^{\prime}, f_{1}^{\prime}\right]=\left[E_{2}^{p-2} E_{1}^{2} e_{2}, f_{1}^{\prime}\right]=E_{2}^{p-2}\left[E_{1}^{2} e_{2}, F_{1}^{p-2} f_{2}\right]=0 .}
\end{aligned}
$$

This completes the proof.

Theorem 3.7. Suppose $\mathfrak{g}=\bigoplus_{t \in \mathbb{Z}} \mathfrak{g}_{t}$ is a $\mathbb{Z}$-graded Lie algebra containing homogeneous elements $e_{i}, f_{i}, i=1,2$, and $h$ satisfying the assumptions of Proposition 3.6. Suppose $e_{i} \in \mathfrak{g}_{\ell_{i}}, f_{i} \in \mathfrak{g}_{-\ell_{i}}$, where $\ell_{1} \ell_{2} \geq 0$ and not both $\ell_{1}$ and $\ell_{2}$ are 0 . Then $\mathfrak{g}$ is infinite-dimensional.

Proof. We may suppose that $e_{i}^{\prime}, f_{i}^{\prime}$ for $i=1,2$ are as in the statement of Proposition 3.6, and that $\left[e_{i}^{\prime}, f_{i}^{\prime}\right]=\zeta_{i} h$ where $\zeta_{i} \neq 0$. Define

$$
\begin{array}{rrrl}
\widetilde{e}_{1} & =2 b^{-1} e_{1}^{\prime}, & \widetilde{f}_{1} & =\zeta_{1}^{-1} f_{1}^{\prime}, \\
\widetilde{e}_{2} & =2 b^{-1} e_{2}^{\prime}, & \widetilde{f}_{2} & =\zeta_{2}^{-1} f_{2}^{\prime}, \\
\widetilde{h} & =\left[\widetilde{e}_{1}, \widetilde{f}_{1}\right]=2 b^{-1} h & =\left[\widetilde{e}_{2}, \widetilde{f}_{2}\right] .
\end{array}
$$


Then

$$
\begin{aligned}
{\left[\widetilde{h}, \widetilde{e}_{1}\right]=2 \widetilde{e}_{1}, } & {\left[\widetilde{h}, \widetilde{f}_{1}\right]=-2 \widetilde{f}_{1}, } \\
{\left[\widetilde{h}, \widetilde{e}_{2}\right]=-2 \widetilde{e}_{2}, } & {\left[\widetilde{h}, \widetilde{f}_{2}\right]=2 \widetilde{f}_{2} . }
\end{aligned}
$$

Moreover, $\widetilde{e}_{1} \in \mathfrak{g}_{k_{1}}, \widetilde{e}_{2} \in \mathfrak{g}_{k_{2}}, \widetilde{f}_{1} \in \mathfrak{g}_{-k_{1}}$, and $\widetilde{f}_{2} \in \mathfrak{g}_{-k_{2}}$, where $k_{1}=$ $-(p-b) \ell_{1}-\ell_{2}, k_{2}=-(p-b) \ell_{1}-3 \ell_{2}$ if $b \in \mathbb{F}_{p}$ and $k_{1}=(p-2) \ell_{1}+\ell_{2}$, $k_{2}=2 \ell_{1}+(p-1) \ell_{2}$ if $b \notin \mathbb{F}_{p}$. Since $k_{1} k_{2} \geq 0$, we may replace the initial set of elements with this new set and continue the process. Since the elements constructed lie in spaces $\mathfrak{g}_{t}$ with $|t|$ increasing at each stage, $\mathfrak{g}$ must be infinite-dimensional.

Theorem 3.8. (Compare $\left[\mathbf{K} 1\right.$, Lem. 20]). Suppose $\mathfrak{g}=\bigoplus_{t \in \mathbb{Z}} \mathfrak{g}_{t}$ is a $\mathbb{Z}$ graded Lie algebra containing nonzero homogeneous elements $e_{i}, f_{i}, h_{i}, i=$ 1,2 , which satisfy $e_{i} \in \mathfrak{g}_{\ell_{i}}, f_{i} \in \mathfrak{g}_{-\ell_{i}}$, where $\ell_{1} \ell_{2} \geq 0$ and not both $\ell_{1}$ and $\ell_{2}$ are 0 . Assume

$$
\left[e_{i}, f_{j}\right]=\delta_{i, j} h_{i}, \quad\left[h_{i}, e_{j}\right]=a_{i, j} e_{j}, \quad\left[h_{i}, f_{j}\right]=-a_{i, j} f_{j},
$$

where $a_{i, j}$ is the $(i, j)$-entry of the matrix

$$
A=\left(\begin{array}{ll}
2 & 0 \\
c & b
\end{array}\right)
$$

and $c \neq 0$. Then $\mathfrak{g}$ is infinite-dimensional.

Proof. We have $\left[f_{2},\left[e_{1}, e_{2}\right]\right]=-\left[e_{1}, h_{2}\right]=c e_{1} \neq 0$, so that $\left[e_{1}, e_{2}\right] \neq 0$. Similarly, $\left[f_{1}, f_{2}\right] \neq 0$. Set $\bar{e}_{1}=e_{1}, \bar{e}_{2}=\left[e_{1}, e_{2}\right], \bar{f}_{1}=f_{1}, \bar{f}_{2}=c^{-1}\left[f_{1}, f_{2}\right]$ and $\bar{h}=h_{1}$. Then we claim these elements satisfy the hypotheses of Proposition 3.6 (with $a=2$ ). All this is apparent except perhaps for the following calculations:

$$
\left[h, \bar{f}_{2}\right]=c^{-1}\left[\left[h_{1}, f_{1}\right], f_{2}\right]=-2 c^{-1}\left[f_{1}, f_{2}\right]=-2 \bar{f}_{2},
$$

and

$$
\begin{aligned}
{\left[\bar{e}_{2}, \bar{f}_{2}\right] } & =c^{-1}\left[\left[e_{1}, e_{2}\right],\left[f_{1}, f_{2}\right]\right] \\
& =c^{-1}\left[\left[\left[e_{1}, e_{2}\right], f_{1}\right], f_{2}\right]+c^{-1}\left[f_{1},\left[\left[e_{1}, e_{2}\right], f_{2}\right]\right] \\
& =c^{-1}\left[\left[h_{1}, e_{2}\right], f_{2}\right]+c^{-1}\left[f_{1},\left[e_{1}, h_{2}\right]\right]=-\left[f_{1}, e_{1}\right]=h_{1}=\bar{h}
\end{aligned}
$$

Since $\left[e_{1}, e_{2}\right] \in \mathfrak{g}_{\ell_{1}+\ell_{2}}$ and $\ell_{1}\left(\ell_{1}+\ell_{2}\right) \geq 0$, we may apply Theorem 3.7 to obtain the desired conclusion.

Corollary 3.9. Suppose that there are nonzero elements $e_{i}, f_{i} \in \mathfrak{g}$, $i=1,2$, and $h$ such that the relations in Proposition 3.6 hold with $a=0$. Assume further that $e_{i} \in \mathfrak{g}_{\ell_{i}}, f_{i} \in \mathfrak{g}_{-\ell_{i}}$, where $\ell_{1} \ell_{2} \geq 0$ and not both $\ell_{1}$ and $\ell_{2}$ are 0 . Then $\mathfrak{g}$ is infinite-dimensional. 
Proof. This is a direct consequence of Theorem 3.8 with $h_{1}=h=h_{2}$, $c=2$, and $b=0$.

Proposition 3.10. Suppose e, $f, h$ span a Heisenberg Lie subalgebra of a Lie algebra $\mathfrak{g}$. Assume $u_{0}, v_{0}$ are elements of $\mathfrak{g}$ such that

$$
\begin{array}{lcc}
{\left[f, u_{0}\right]=0,} & {\left[h, u_{0}\right]=-\lambda u_{0},} & \\
{\left[e, v_{0}\right]=0,} & {\left[h, v_{0}\right]=\lambda v_{0},} & \\
{\left[u_{0}, v_{0}\right]=z,} & {\left[z, v_{0}\right]=2 v_{0},} & {\left[z, u_{0}\right]=-2 u_{0},} \\
{[z, f]=-\eta f,} & {[z, e]=\eta e} & (\text { some } \eta \in \mathbb{F}) .
\end{array}
$$

Then

$$
\begin{aligned}
& {\left[E^{\ell} u_{0}, F^{\ell} v_{0}\right]=(-1)^{\ell} \ell ! \lambda^{\ell-1}(\lambda z-\ell \eta h)} \\
& {\left[\left[E^{\ell} u_{0}, F^{\ell} v_{0}\right], E^{\ell} u_{0}\right]=2(-1)^{\ell} \ell ! \lambda^{\ell}(\ell \eta-1) E^{\ell} u_{0}} \\
& {\left[\left[E^{\ell} u_{0}, F^{\ell} v_{0}\right], F^{\ell} v_{0}\right]=-2(-1)^{\ell} \ell ! \lambda^{\ell}(\ell \eta-1) F^{\ell} v_{0}}
\end{aligned}
$$

for all $\ell \in\{1, \ldots, p-1\}$.

Proof. Following the argument in (3.5), we have

$$
\begin{aligned}
{\left[E^{\ell} u_{0}, F^{\ell} v_{0}\right] } & =\sum_{k=0}^{\ell}(-1)^{k}\left(\begin{array}{l}
\ell \\
k
\end{array}\right) E^{\ell-k} U_{0} E^{k} F^{\ell} v_{0} \\
& =(-1)^{\ell-1} \ell \ell ! \lambda^{\ell-1} E U_{0} F v_{0}+(-1)^{\ell} \ell ! \lambda^{\ell} U_{0} v_{0} \\
& =(-1)^{\ell} \ell ! \lambda^{\ell-1}(\lambda z-\ell \eta h) .
\end{aligned}
$$

Since $\left[\lambda z-\ell \eta h, E^{\ell} u_{0}\right]=((\ell \eta-2) \lambda+\ell \eta \lambda) E^{\ell} u_{0}=2 \lambda(\ell \eta-1) E^{\ell} u_{0}$, equation (3.13) follows, and (3.14) is completely analogous.

\subsection{Primitive vectors in $\mathfrak{g}_{1}$ and $\mathfrak{g}_{-1}$}

In this section, we show that if $e$ is a $\mathfrak{b}^{+}$-primitive vector in $\mathfrak{g}_{1}$, and $f$ is a $\mathfrak{b}^{-}$-primitive vector in $\mathfrak{g}_{-1}$, then $[e,[e, f]] \neq 0$, and $e$ and $f$ generate a three-dimensional simple Lie algebra.

Henceforth in this section we assume the following:

(a) $\mathfrak{g}=\bigoplus_{j \in \mathbb{Z}} \mathfrak{g}_{j}$ is a finite-dimensional $\mathbb{Z}$-graded Lie algebra over an algebraically closed field of characteristic $p>3$.

(b) $\mathfrak{g}_{0}$ is classical reductive, and $\mathfrak{t}$ is a maximal toral subalgebra of $\mathfrak{g}_{0}$.

(c) $\left\{\alpha_{1}, \ldots, \alpha_{m}\right\}$ is a base of simple roots relative to $\mathfrak{t}$,

(d) $e_{i} \in \mathfrak{g}_{0}^{\alpha_{i}}, f_{i} \in \mathfrak{g}_{0}^{-\alpha_{i}}$ denote nonzero root vectors corresponding to these simple roots.

(e) $\mathfrak{b}^{+}=\mathfrak{t} \oplus \mathfrak{n}^{+}$where $\mathfrak{n}^{+}=\bigoplus_{\alpha>0} \mathfrak{g}_{0}^{\alpha}$, and $\mathfrak{b}^{-}=\mathfrak{t} \oplus \mathfrak{n}^{-}$where $\mathfrak{n}^{-}=$ $\bigoplus_{\alpha>0} \mathfrak{g}_{0}^{-\alpha}$. 
(f) the representation of $\mathfrak{g}_{0}^{(1)}:=\left[\mathfrak{g}_{0}, \mathfrak{g}_{0}\right]$ on $\mathfrak{g}_{-1}$ is irreducible and restricted.

Assumption (f) implies that $\mathfrak{g}_{-1}$ possesses $\mathfrak{b}^{-}$-primitive vector $f \neq 0$. Thus, there exists $\delta \in \mathfrak{t}^{*}$ such that $[t, f]=\delta(t) f$ for all $t \in \mathfrak{t}$ and $[y, f]=0$ for all $y \in \mathfrak{n}^{-}$. If $\mathfrak{g}$ is transitive (1.2), then $\mathfrak{g}_{k}$ is a restricted module for $\mathfrak{g}_{0}^{(1)}$ for all $k \geq 0$. In particular, $\mathfrak{g}_{1}$ will possess a $\mathfrak{b}^{+}$-primitive vector $e \neq 0$. Thus, there will be $\gamma \in \mathfrak{t}^{*}$ such that $[t, e]=\gamma(t) e$ for all $t \in \mathfrak{t}$ and $[x, e]=0$ for all $x \in \mathfrak{n}^{+}$.

Lemma 3.15. Assume $\mathfrak{g}$ is transitive (1.2) and e and $f$ are as in Section 3.3. Then $[e, f] \neq 0$.

Proof. Suppose the contrary. Then since $\mathfrak{g}_{-1}$ is irreducible, it is spanned by vectors of the form $\left[e_{i_{k}} \cdots,\left[e_{i_{2}},\left[e_{i_{1}}, f\right]\right] \ldots\right], k \geq 0$, and

$$
\left[e,\left[e_{i_{k}} \cdots,\left[e_{i_{2}},\left[e_{i_{1}}, f\right]\right] \ldots\right]\right]=\left[e_{i_{k}} \cdots,\left[e_{i_{2}},\left[e_{i_{1}},[e, f]\right]\right] \ldots\right]=0 .
$$

By transitivity, $e=0$, a contradiction.

Lemma 3.16. If $f$ and $e$ are as in Section 3.3 and $\delta=-\gamma$, then $[[e, f], f]$ $=0$ if and only if $[[e, f], e]=0$.

Proof. Clearly, $[[e, f], f]=0$ if and only if $\gamma([e, f])=0$ if and only if $[[e, f], e]=0$.

THEOREM 3.17. Let $\mathfrak{g}$ be a graded Lie algebra satisfying $(a)-(f)$ of Section 3.3, and assume that $\mathfrak{g}$ is transitive (1.2). Let $e$ and $f$ be as in Section 3.3, and suppose that $\delta=-\gamma$. Then $[[e, f], e] \neq 0$.

Proof. We begin by assuming the conclusion is false. Observe that then both $[[e, f], f]$ and $[[e, f], e]$ are 0 by Lemma 3.16, and $h=[e, f]$ is nonzero by Lemma 3.15, so that $e, f, h$ span a Heisenberg Lie algebra. If $\alpha_{j}(h)=0$ for all simple roots $\alpha_{j}$, then $h \in \mathfrak{t}$ belongs to the center of $\mathfrak{g}_{0}$. But then $h$ acts as a nonzero scalar $\zeta$ on $\mathfrak{g}_{-1}$ by Schur's Lemma and transitivity. That would imply $[[e, f], f]=\zeta f \neq 0$. Consequently, there must exist some simple root $\alpha_{k}$ such that $\alpha_{k}(h) \neq 0$. We suppose that $e_{k}$ and $f_{k}$ are root vectors corresponding to $\alpha_{k}$ and $-\alpha_{k}$ respectively, chosen so that $e_{k}, f_{k}, h_{k}=\left[e_{k}, f_{k}\right]$ form a canonical basis for $\mathfrak{s l}_{2}$.

Let us set $u_{0}=-f_{k}, v_{0}=e_{k}$, and $z=h_{k}$. Then the relations in (3.11) hold,

$$
\begin{array}{lll}
{\left[f, u_{0}\right]=0,} & {\left[h, u_{0}\right]=-\lambda u_{0},} & \\
{\left[e, v_{0}\right]=0,} & {\left[h, v_{0}\right]=\lambda v_{0},} & \\
{\left[u_{0}, v_{0}\right]=z,} & {\left[z, v_{0}\right]=2 v_{0},} & {\left[z, u_{0}\right]=-2 u_{0},} \\
{[z, f]=-\eta f,} & {[z, e]=\eta e,} &
\end{array}
$$


where $\lambda=\alpha_{k}(h) \neq 0$ and $\eta=\gamma\left(h_{k}\right)$. Note that by replacing $f$ by $\lambda^{-1} f$ and $h$ by $\lambda^{-1} h$, we may suppose that $\lambda=1$. We claim $\eta \neq 0$. If $\eta=0$, then for

$$
\begin{array}{ccc}
\tilde{e}_{1}=-u_{0}=f_{k}, & \tilde{f}_{1}=v_{0}=e_{k}, & \widetilde{h}_{1}=-h_{k}=\left[f_{k}, e_{k}\right]=-z \\
\widetilde{e}_{2}=e, & \widetilde{f}_{2}=f, & \widetilde{h}_{2}=h=[e, f],
\end{array}
$$

we have the relations

$$
\left[\widetilde{e}_{i}, \widetilde{f}_{j}\right]=\delta_{i, j} \widetilde{h}_{i}, \quad\left[\widetilde{h}_{i}, \widetilde{e}_{j}\right]=a_{i, j} \widetilde{e}_{j}, \quad\left[\widetilde{h}_{i}, \widetilde{f}_{j}\right]=-a_{i, j} \widetilde{f}_{j}
$$

where $a_{i, j}$ is the $(i, j)$-entry of the matrix

$$
A=\left(\begin{array}{cc}
2 & -\eta \\
-1 & 0
\end{array}\right)
$$

When $\eta=0$, then by Theorem 3.8 we would have that $\mathfrak{g}$ is infinite-dimensional. Therefore, $\eta \neq 0$. Note also that since $\mathfrak{g}_{-1}$ is a restricted $\mathfrak{g}_{0}^{(1)}$-module, $\eta \in \mathbb{F}_{p}$.

Because the relations in (3.11) hold, we can now apply Proposition 3.10 to get (3.12)-(3.14):

$$
\begin{aligned}
& {\left[E^{\ell} u_{0}, F^{\ell} v_{0}\right]=(-1)^{\ell} \ell !(z-\ell \eta h),} \\
& {\left[\left[E^{\ell} u_{0}, F^{\ell} v_{0}\right], E^{\ell} u_{0}\right]=2(-1)^{\ell} \ell !(\ell \eta-1) E^{\ell} u_{0}, \quad \text { and }} \\
& {\left[\left[E^{\ell} u_{0}, F^{\ell} v_{0}\right], F^{\ell} v_{0}\right]=-2(-1)^{\ell} \ell !(\ell \eta-1) F^{\ell} v_{0}}
\end{aligned}
$$

for all $\ell \in\{1, \ldots, p-1\}$.

We suppose first that $\eta=1$. Here we exploit the fact that now

$$
\left[\left[E u_{0}, F v_{0}\right], E u_{0}\right]=-2(\eta-1) E u_{0}=0,
$$

so that $E u_{0}$ and $F v_{0}$ span a Heisenberg algebra. We set

$$
\begin{array}{ccc}
e^{\prime}=E u_{0}, & f^{\prime}=F v_{0}, & h^{\prime}=h-z, \\
u_{0}^{\prime}=E^{\ell} u_{0}, & v_{0}^{\prime}=c F^{\ell} v_{0}, & z^{\prime}=(-1)^{\ell} \ell ! c(z-\ell h),
\end{array}
$$

for an arbitrary but fixed nonzero $c \in \mathbb{F}$. Then in view of the relations above, we have for $3 \leq \ell \leq p-1$ that 


$$
\begin{aligned}
{\left[f^{\prime}, u_{0}^{\prime}\right] } & =\left[\left[f, v_{0}\right], E^{\ell} u_{0}\right]=0 \\
{\left[e^{\prime}, v_{0}^{\prime}\right] } & =0 \\
{\left[h^{\prime}, u_{0}^{\prime}\right] } & =\left[h-z, E^{\ell} u_{0}\right]=(1-\ell) u_{0}^{\prime} \neq 0 \\
{\left[h^{\prime}, v_{0}^{\prime}\right] } & =(\ell-1) v_{0}^{\prime} \\
{\left[u_{0}^{\prime}, v_{0}^{\prime}\right] } & =z^{\prime} \\
{\left[z^{\prime}, u_{0}^{\prime}\right] } & =2(-1)^{\ell} \ell ! c(\ell-1) u_{0}^{\prime} \\
{\left[z^{\prime}, v_{0}^{\prime}\right] } & =-2(-1)^{\ell} \ell ! c(\ell-1) v_{0}^{\prime} \\
{\left[z^{\prime}, f^{\prime}\right] } & =(-1)^{\ell} \ell ! c\left[z-\ell h,\left[f, v_{0}\right]\right]=(-1)^{\ell} \ell ! c(1-\ell) f^{\prime} \\
{\left[z^{\prime}, e^{\prime}\right] } & =(-1)^{\ell} \ell ! c(\ell-1) e^{\prime} .
\end{aligned}
$$

Taking $c$ so $c^{-1}=(-1)^{\ell-1} \ell !(\ell-1)$, we get that the primed letters satisfy all the relations in (3.11) with $\lambda^{\prime}=\ell-1$ and $\eta^{\prime}=-1 \equiv p-$ $1 \bmod p$. Consequently, by replacing $e, f, h, u_{0}, v_{0}, z$ by the corresponding primed letters, we may suppose that $\eta \neq 0,1$.

Then setting $\ell=\eta^{-1}$, we see that the right side of (3.13) becomes 0 . The expression in (3.12) is nonzero since $z$ and $h$ are linearly independent due to the fact $[z, e]=\eta e \neq 0$, while $[h, e]=0$. Since $\ell>1$, the vector $\widetilde{e}=E^{\ell} u_{0}$ (resp. $\left.\widetilde{f}=F^{\ell} v_{0}\right)$ is a $\mathfrak{b}^{+}$-primitive (resp. $\mathfrak{b}^{-}$-primitive) vector. Moreover the properties that pertain to $e$ and $f$, namely $[\widetilde{e}, \widetilde{f}] \neq 0$ and $[[\widetilde{e}, \widetilde{f}], \widetilde{f}]=0=[[\widetilde{e}, \widetilde{f}], \widetilde{e}]$ hold for them. Note also that $\widetilde{h}:=[\widetilde{e}, \widetilde{f}]$ is not central. Indeed, if $\widetilde{h} \in \mathfrak{Z}\left(\mathfrak{g}_{0}\right)$, then it acts as a scalar, say $\kappa \neq 0$, on $\mathfrak{g}_{-1}$, and as $\ell \kappa$ on $\left(\mathfrak{g}_{-1}\right)^{\ell}$. But then $[\widetilde{h}, \widetilde{f}]=\ell \kappa \widetilde{f}$, a contradiction, since $\kappa \neq 0$ and $\ell=\eta^{-1} \neq 0$, but $[[\widetilde{e}, \widetilde{f}], \widetilde{f}]=0$. We may continue the argument with this new triple $\widetilde{e}, \widetilde{f}, \widetilde{h}$ of elements. Since when $f \in \mathfrak{g}_{-i}$, then $\widetilde{f} \in \mathfrak{g}_{-i}^{n}$ for some $n \in\{2, \ldots, p-1\}$, the elements produced belong to the spaces $\mathfrak{g}_{t}$ with $|t|$ increasing. Thus, $\mathfrak{g}$ must be infinite-dimensional. We have reached a contradiction. Thus, $[[e, f], e] \neq 0$.

\subsection{Subalgebras with a balanced grading}

We consider graded Lie algebras $\mathfrak{g}=\bigoplus_{j=-q}^{r} \mathfrak{g}_{j}$ with $\mathfrak{g}_{1}$ contragredient (as a $\mathfrak{g}_{0}$-module) to $\mathfrak{g}_{-1}$. When $\mathfrak{g}$ is generated by its local part $\mathfrak{g}_{-1} \oplus \mathfrak{g}_{0} \oplus \mathfrak{g}_{1}$, we prove that $\mathfrak{g}$ must have a balanced gradation (i.e. $q=r$ ) and then argue that $\mathfrak{g}$ must be classical. For example, the Lie subalgebra of the Melikyan algebra $M(2 ; \underline{n})$ generated by the local part is a simple classical Lie algebra of type $\mathrm{G}_{2}$.

Our assumptions for the remainder of Chapter 3 are the following:

(1) $\mathfrak{g}=\bigoplus_{j=-q}^{r} \mathfrak{g}_{j}$ is a graded Lie algebra over an algebraically closed field $\mathbb{F}$ of characteristic $p>3$; 
(2) $\mathfrak{g}_{0}$ is classical reductive;

(3) $\mathfrak{g}$ is transitive (1.2) and 1-transitive (1.3);

(4) $\mathfrak{g}_{-1}$ is an irreducible restricted module for $\mathfrak{g}_{0}^{(1)}=\left[\mathfrak{g}_{0}, \mathfrak{g}_{0}\right]$;

(5) $\mathfrak{g}_{1}$ is a $\mathfrak{g}_{0}$-module isomorphic to the dual module $\mathfrak{g}_{-1}^{*}$.

(6) $\mathfrak{t}$ is a maximal toral subalgebra of $\mathfrak{g}_{0} ; \mathfrak{b}^{+}=\mathfrak{t} \oplus \mathfrak{n}^{+}$where $\mathfrak{n}^{+}=$ $\bigoplus_{\alpha>0} \mathfrak{g}_{0}^{\alpha} ;$ and $\mathfrak{b}^{-}=\mathfrak{t} \oplus \mathfrak{n}^{-}$where $\mathfrak{n}^{-}=\bigoplus_{\alpha>0} \mathfrak{g}_{0}^{-\alpha}$.

Let $f^{\Lambda} \in \mathfrak{g}_{-1}$ be a $\mathfrak{b}^{+}$-primitive vector of weight $\Lambda$ in the irreducible restricted $\mathfrak{g}_{0}^{(1)}$-module $\mathfrak{g}_{-1}$. We assume $e^{-\Lambda} \in \mathfrak{g}_{1}$ is a $\mathfrak{b}^{-}$-primitive vector of weight $-\Lambda$ in the irreducible $\mathfrak{g}_{0}^{(1)}$-module $\mathfrak{g}_{1}$. Since the Lie algebra $\mathfrak{g}_{0}$ is classical reductive, it has a presentation by generators $e_{i}, f_{i}, i \in \mathrm{I}, h_{j}, j \in \mathrm{J}$, and Serre relations, (where $\mathrm{I}=\mathrm{J}$ if no ideal summands of the form $\mathfrak{Z}\left(\mathfrak{g}_{0}\right)$, or $\mathfrak{g l}_{n}, \mathfrak{s l}_{n}$ or $\mathfrak{p g l}_{n}$ with $p \mid n$ occur). There is an automorphism $\sigma$ on $\mathfrak{g}_{0}$ such that $\sigma\left(f_{i}\right)=e_{i}, \sigma\left(e_{i}\right)=f_{i}, i \in \mathrm{I}$, and $\sigma\left(h_{j}\right)=-h_{j}, j \in \mathrm{J}$.

Since the $\mathfrak{g}_{0}$-modules $\mathfrak{g}_{1}$ and $\mathfrak{g}_{-1}$ are dual to one another, there is a $\mathfrak{g}_{0}$-homomorphism $\phi: \mathfrak{g}_{-1} \otimes \mathfrak{g}_{1} \rightarrow \mathfrak{g}_{0}$ given by $\phi(u \otimes v)=[u, v]$. Let $\mathfrak{F}^{ \pm}$be the free Lie algebra generated by $\mathfrak{g}_{ \pm 1}$, and set $\mathfrak{F}=\mathfrak{F}^{-} \oplus \mathfrak{F}_{0} \oplus \mathfrak{F}^{+}$, where $\mathfrak{F}_{0}=\mathfrak{g}_{0}$. Then $\mathfrak{F}$ is a Lie algebra (compare $[\mathbf{B K M}]$ ), and it is generated by the elements $e_{i}, f_{i}, i \in \mathrm{I}, h_{j}, j \in \mathrm{J}, f^{\Lambda}, e^{-\Lambda}$. We may assign a grading to $\mathfrak{F}$ by setting $0=\operatorname{deg}\left(e_{i}\right)=\operatorname{deg}\left(f_{i}\right)=\operatorname{deg}\left(h_{j}\right)$ for all $i, j$ and $\operatorname{deg}\left(f^{\Lambda}\right)=-1=$ $-\operatorname{deg}\left(e^{-\Lambda}\right)$ so that $\mathfrak{F}^{ \pm}=\sum_{t=1}^{\infty} \mathfrak{F}_{ \pm t}$ where $\mathfrak{F}_{ \pm t}$ is the span of the elements of degree $\pm t$. We claim we can extend the automorphism $\sigma$ on $\mathfrak{g}_{0}=\mathfrak{F}_{0}$ to one on $\mathfrak{F}$. Indeed, first define a new $\mathfrak{g}_{0}$-module structure on $\mathfrak{g}_{-1}$ by $x * v=[\sigma(x), v]$. Then $f_{i} * f^{\Lambda}=\left[e_{i}, f^{\Lambda}\right]=0$ and $h_{j} * f^{\Lambda}=-\left[h_{j}, f^{\Lambda}\right]=-\Lambda\left(h_{j}\right) f^{\Lambda}$. It follows from these calculations that $\mathfrak{g}_{-1}$ with this new $\mathfrak{g}_{0}$-action is an irreducible $\mathfrak{g}_{0}$-module with a $\mathfrak{b}^{-}$-primitive vector of weight $-\Lambda$. Since such a module is unique up to isomorphism, we can define a $\mathfrak{g}_{0}$-module isomorphism $\sigma$ : $\mathfrak{g}_{-1} \rightarrow \mathfrak{g}_{1}$ so that $\sigma\left(f^{\Lambda}\right)=e^{-\Lambda}$. The same argument shows that $\mathfrak{g}_{1}$ under the $\mathfrak{g}_{0}$-action $x * u=[\sigma(x), u]$ is a $\mathfrak{g}_{0}$-module with a $\mathfrak{b}^{+}$-primitive vector of weight $\Lambda$. Thus we can define a $\mathfrak{g}_{0}$-module isomorphism $\sigma: \mathfrak{g}_{1} \rightarrow \mathfrak{g}_{-1}$ so that $\sigma\left(e^{-\Lambda}\right)=f^{\Lambda}$. Note then that

$$
\sigma([x, w])=\sigma(\sigma(x) * w)=[\sigma(x), \sigma(w)]
$$

for all $x \in \mathfrak{g}_{0}$ and $w \in \mathfrak{g}_{-1}$ or $w \in \mathfrak{g}_{1}$. We may now extend $\sigma$ to be an automorphism on the free Lie algebras $\mathfrak{F}^{+}$and $\mathfrak{F}^{-}$so that (3.19) holds for all $x \in \mathfrak{F}_{0}=\mathfrak{g}_{0}$ and $w \in \mathfrak{F}^{+}$or $w \in \mathfrak{F}^{-}$.

Let $\widehat{\mathfrak{g}}$ denote the Lie subalgebra of $\mathfrak{g}$ generated by the local part $\mathfrak{g}_{-1} \oplus$ $\mathfrak{g}_{0} \oplus \mathfrak{g}_{1}$. There is a natural epimorphism $\phi: \mathfrak{F} \rightarrow \widehat{\mathfrak{g}}$ taking the generators to the corresponding elements in $\widehat{\mathfrak{g}}$. The kernel is a homogeneous ideal, $\mathfrak{K}=\bigoplus_{t \in \mathbb{Z}} \mathfrak{K}_{t}$ of $\mathfrak{F}$, which trivially intersects the local part $\mathfrak{F}_{-1} \oplus \mathfrak{F}_{0} \oplus \mathfrak{F}_{1}$.

Suppose $m \geq 2$ is minimal with $\mathfrak{K}_{m} \neq 0$. Let $y \in \mathfrak{K}_{m}$. Then $\left[y, \mathfrak{F}_{-1}\right]=0$ by the minimality of $m$. Hence $\left[\sigma(y), \mathfrak{F}_{1}\right]=0$, and applying $\phi$ we have $\left[\phi(\sigma(y)), \mathfrak{g}_{1}\right]=0$. By the 1-transitivity of $\mathfrak{g}$, we must have $\phi(\sigma(y))=0$; that 
is, $\sigma(y) \in \mathfrak{K}_{-m}$. Thus, $\sigma: \mathfrak{K}_{m} \rightarrow \mathfrak{K}_{-m}$. Arguing with the minimal $n$ such that $\mathfrak{K}_{-n} \neq 0$ shows that $\sigma: \mathfrak{K}_{-n} \rightarrow \mathfrak{K}_{n}$. Thus, these two minimal values $m, n$ must be equal, and $\sigma$ must interchange the subspaces $\mathfrak{K}_{m}$ and $\mathfrak{K}_{-m}$. Suppose we have shown that $\sigma$ interchanges $\mathfrak{K}_{s}$ and $\mathfrak{K}_{-s}$ for all $2 \leq m \leq$ $s<t$. Then $\left[\mathfrak{K}_{t}, \mathfrak{F}_{-1}\right] \subseteq \mathfrak{K}_{t-1}$, and $\left[\sigma\left(\mathfrak{K}_{t}\right), \mathfrak{F}_{1}\right] \subseteq \mathfrak{K}_{-(t-1)}$. Applying $\phi$ gives $\left[\phi\left(\sigma\left(\mathfrak{K}_{t}\right)\right), \mathfrak{g}_{1}\right] \subseteq \phi\left(\mathfrak{K}_{-(t-1)}\right)=0$. Once again by 1 -transitivity, it must be that $\sigma\left(\mathfrak{K}_{t}\right) \subseteq \mathfrak{K}_{-t}$. Replacing $t$ with $-t$ and -1 with 1 in the argument gives $\sigma\left(\mathfrak{K}_{-t}\right) \subseteq \mathfrak{K}_{t}$. Since $\sigma$ is an automorphism, equality must hold. Thus, $\mathfrak{K}$ is $\sigma$-invariant, so that there is an induced automorphism $\sigma$ on $\mathfrak{F} / \mathfrak{K} \cong \widehat{\mathfrak{g}}$. This says that the gradation of $\widehat{\mathfrak{g}}$ is balanced. We now summarize what we have just shown.

TheOREm 3.20. Assume $\mathfrak{g}=\bigoplus_{j=-q}^{r} \mathfrak{g}_{j}$ is a graded Lie algebra satisfying (1)-(5) of Section 3.4. Then the gradation of the subalgebra $\widehat{\mathfrak{g}}$ of $\mathfrak{g}$ generated by the local part $\mathfrak{g}_{-1} \oplus \mathfrak{g}_{0} \oplus \mathfrak{g}_{1}$ is balanced.

We now come to the first main result of this section.

THEOREM 3.21. Assume $\mathfrak{g}=\bigoplus_{j=-q}^{r} \mathfrak{g}_{j}$ is a graded Lie algebra satisfying (1)-(5) of Section 3.4. Then the graded Lie subalgebra $\hat{\mathfrak{g}}$ generated by the local part $\mathfrak{g}_{-1} \oplus \mathfrak{g}_{0} \oplus \mathfrak{g}_{1}$ of $\mathfrak{g}$ is either classical simple or isomorphic to $\mathfrak{p g l}_{k p}$ for some $k \in \mathbb{N}$.

Proof. The Lie algebra $\widehat{\mathfrak{g}}$ generated by $\mathfrak{g}_{-1} \oplus \mathfrak{g}_{0} \oplus \mathfrak{g}_{1}$ satisfies all the hypotheses of the theorem, so we will assume from now that $\mathfrak{g}=\widehat{\mathfrak{g}}$. Let $S:=\bigoplus_{i \geq 0}\left(\operatorname{ad} \mathfrak{g}_{1}\right)^{i} \mathfrak{g}_{-q}$. Since $\mathfrak{g}$ is generated by its local part, Lemma 1.16 shows that $S$ is the unique minimal ideal of $\mathfrak{g}$. This, in turn, implies that $\operatorname{Ann}_{\mathfrak{g}} S=0$. In particular, the center $\mathfrak{Z}(S)=0$ and $\mathfrak{g}$ can be regarded as a Lie subalgebra of $\operatorname{Der}(S)$. Let $G$ denote the connected component of the automorphism group of the Lie algebra $S$, and let $\mathcal{L}$ denote the Lie algebra of the algebraic group $G$. Then $\mathcal{L}$ is a Lie subalgebra of $\operatorname{Der}(S)$.

(a) Suppose we have established that ad $S \subseteq \mathcal{L}$. Then ad $S$ is an ideal of $\mathcal{L} \subseteq \operatorname{Der}(S)$. Let $R$ denote the solvable radical of the algebraic group $G$ and $\mathcal{R}=\operatorname{Lie}(R)$, a solvable subalgebra of $\operatorname{Der}(S)$. Since $\mathcal{R}$ is an ideal of $\mathcal{L}$, the subspace $[\mathcal{R}, \operatorname{ad} S]$ is a solvable ideal of ad $S$. Let $\mathfrak{r}$ denote the inverse image of $[\mathcal{R}, \operatorname{ad} S]$ under the isomorphism ad : $S \stackrel{\sim}{\longrightarrow}$ ad $S$.

Suppose $\mathcal{R} \neq 0$. Then $[\mathcal{R}, \operatorname{ad} S] \neq 0$ and hence $\mathfrak{r} \cap \mathfrak{g}_{-1} \neq 0$ by the transitivity of $\mathfrak{g}$. The irreducibility of $\mathfrak{g}_{-1}$ shows that the $\mathfrak{g}_{0}$-module $\{x \in$ $\left.\mathfrak{g}_{-1} \mid\left[x, \mathfrak{g}_{1}\right]=0\right\}$ is zero. This, in turn, shows that $J:=\left[\mathfrak{r} \cap \mathfrak{g}_{-1}, \mathfrak{g}_{1}\right]$ is a nonzero solvable ideal of $\left[\mathfrak{g}_{-1}, \mathfrak{g}_{1}\right]$. Since $\mathfrak{g}_{0}$ acts irreducibly and faithfully on $\mathfrak{g}_{-1}$, Schur's lemma implies that $\mathfrak{Z}\left(\mathfrak{g}_{0}\right)$ acts on $\mathfrak{g}_{-1}$ as scalar operators, so that $\operatorname{dim} \mathfrak{Z}\left(\mathfrak{g}_{0}\right) \leq 1$ (see the discussion in Section 1.10). Lemma 1.71 now says that $J \subseteq \mathfrak{Z}\left(\mathfrak{g}_{0}\right)$ (one should keep in mind that $\left[\mathfrak{g}_{-1}, \mathfrak{g}_{1}\right]$ is an ideal of $\left.\mathfrak{g}_{0}\right)$. Since $J \neq 0$, there is $z \in \mathcal{R}$ such that $z(x)=x$ for all $x \in \mathfrak{g}_{-1}$. Since $\mathfrak{g}_{1}$ 
is isomorphic to a $\mathfrak{g}_{0}$-submodule of $\operatorname{Hom}\left(\mathfrak{g}_{-1}, \mathfrak{g}_{0}\right)$, we have $z(x)=-x$ for all $x \in \mathfrak{g}_{1}$. But then ad $\mathfrak{g}_{ \pm 1} \subset \mathcal{R}$, contradicting the solvability of $\mathcal{R}$. We thus deduce that $\mathcal{R}=0$. Consequently, $G$ is a semisimple (connected) algebraic group.

Because the center $Z(G)$ of the group $G$ acts trivially on $\mathcal{L}$, it also acts trivially on ad $S \subseteq \mathcal{L}$. Since $\mathfrak{Z}(S)=0$, it follows that the scheme $Z(G)$ is trivial, i.e., $G$ is a semisimple group of adjoint type. It follows that $G$ is isomorphic (as an algebraic group) to the direct product of its simple components $G_{1}, \ldots, G_{d}$. As a consequence, $\mathcal{L}=\mathcal{L}_{1} \oplus \cdots \oplus \mathcal{L}_{d}$ where $\mathcal{L}_{i}=\operatorname{Lie}\left(G_{i}\right)$ (a direct sum of Lie algebras). Since $\left[\mathcal{L}_{i}\right.$, ad $\left.S\right] \subseteq$ ad $S$ is a nonzero ideal of ad $S$ and $\mathcal{L}_{i} \cap \mathcal{L}_{j}=0$ for $i \neq j$, the simplicity of $S$ forces $d=1$. In other words, $G$ is a simple algebraic group of adjoint type. But then either $\mathcal{L}$ is a classical simple Lie algebra or $\mathcal{L} \cong \mathfrak{p g l}_{k p}$ for some $k \in \mathbb{N}$. Since ad $S$ is a simple ideal of $\mathcal{L}$, we derive that $S$ is classical simple. If $S \neq \mathfrak{p s l}_{k p}$, then ad $S=\operatorname{Der}(S)$, hence $\mathfrak{g} \hookrightarrow \operatorname{Der}(S)$ is classical simple. If $S \cong \mathfrak{p s l}_{k p}$ for some $k \in \mathbb{N}$, then ad $S$ has codimension 1 in $\operatorname{Der}(S) \cong \mathfrak{p g l}_{k p}$. Therefore, in any event either $\mathfrak{g}$ is classical simple or $\mathfrak{g} \cong \mathfrak{p g l}_{k p}$, as wanted.

(b) By Theorem 3.20, $r=q$. The argument used in the proof of Lemma 2.8 shows that $\exp (\operatorname{tad} x) \in G$ for all $x \in S$ such that $(\operatorname{ad} x)^{(p+1) / 2}=0$ and all $t \in \mathbb{F}$. From this it follows that for all such $x$ we have that ad $x \in \mathcal{L}$. Since $p>3$ and $\left(\operatorname{ad} \mathfrak{g}_{ \pm q}\right)^{3}(S)=0$, this implies that ad $\mathfrak{g}_{ \pm q} \subset \mathcal{L}$.

If $q=1$, then

$$
\operatorname{ad} S=\operatorname{ad} \mathfrak{g}_{-1} \oplus\left[\operatorname{ad} \mathfrak{g}_{-1}, \operatorname{ad} \mathfrak{g}_{1}\right] \oplus\left[\left[\operatorname{ad} \mathfrak{g}_{-1}, \operatorname{ad} \mathfrak{g}_{1}\right], \operatorname{ad} \mathfrak{g}_{1}\right] \subseteq \mathcal{L},
$$

and the statement follows from part (a) of this proof. Thus in what follows we may assume that $q \geq 2$.

Suppose ad $\mathfrak{g}_{q-1} \subset \mathcal{L}$. Then ad $\mathfrak{g}_{-1}=\left[\operatorname{ad} \mathfrak{g}_{-q}, \mathfrak{g}_{q-1}\right] \subset \mathcal{L}$, and hence ad $S \subseteq \mathcal{L}$ thanks to Lemmas 1.16 and 1.46.

(c) Suppose $q \geq 3$. If $p>5$, then

$$
\left(\operatorname{ad} \mathfrak{g}_{q-1}\right)^{(p+1) / 2}(\mathfrak{g}) \subseteq\left(\operatorname{ad} \mathfrak{g}_{q-1}\right)^{4}(S) \subseteq \mathfrak{g}_{3 q-4}=0,
$$

which shows that ad $\mathfrak{g}_{q-1} \subset \mathcal{L}$. Then our remarks in part (b) imply that $\mathfrak{g}$ is either classical simple or is isomorphic to $\mathfrak{p g l}_{k p}$. If $p=5$ and $q \geq 4$, then $\left(\operatorname{ad} \mathfrak{g}_{q-1}\right)^{(p+1) / 2}(S) \subseteq \mathfrak{g}_{2 q-3}=0$. Again the result follows by part (b).

Suppose $p=5$ and $q=3$. Given $x \in \mathfrak{g}_{q-1}=\mathfrak{g}_{2}$ set $X:=\operatorname{ad} x$. Clearly, $X^{4}=0$. As $X^{2}(S) \subset \mathfrak{g}_{1} \oplus \mathfrak{g}_{2} \oplus \mathfrak{g}_{3}$ and $X^{3}(S) \subset \mathfrak{g}_{3}$, it must be that $\left[X^{3}(S), X^{2}(S)\right]=0$. By (the proof of) Lemma 2.8 , $\exp t X \in G$ for all $t \in \mathbb{F}$. But then again ad $\mathfrak{g}_{q-1} \subset \mathcal{L}$ and we are done by part (b).

Suppose $p>5$ and $q=2$. For $x \in \mathfrak{g}_{1}$ we now have $X^{4}(S) \subseteq \mathfrak{g}_{2}$ and $X^{3}(S) \subset \mathfrak{g}_{1} \oplus \mathfrak{g}_{2}$. Also, $X^{5}=0$. Hence $\exp t X \in G$ for all $t \in \mathbb{F}$ provided that $p>7$. If $p=7$, then $\left[X^{i}(S), X^{p-i}(S)\right] \subseteq\left[\mathfrak{g}_{1} \oplus \mathfrak{g}_{2}, \mathfrak{g}_{2}\right]=0$, and again $\exp t X \in G$ for all $t \in \mathbb{F}$. Thus this case can be argued as in part (b). 
(d) Finally, suppose $q=2$ and $p=5$. This case is much more complicated. We now have

$$
\mathfrak{g}=\mathfrak{g}_{-2} \oplus \mathfrak{g}_{-1} \oplus \mathfrak{g}_{0} \oplus \mathfrak{g}_{1} \oplus \mathfrak{g}_{2} .
$$

Recall that $\mathfrak{g}$ is irreducible and transitive. Therefore, $\mathfrak{g}_{-2}$ is an irreducible $\mathfrak{g}_{0}$-module and $\left[\left[\mathfrak{g}_{-2}, \mathfrak{g}_{2}\right], \mathfrak{g}_{-2}\right]=\mathfrak{g}_{-2} ;$ see Lemmas $1.41(\mathrm{~b})$ and 1.49.

Let $A_{0}:=\operatorname{Ann}_{\mathfrak{g}_{0}}\left(\mathfrak{g}_{-2}\right)$ and $A_{2}:=\left\{x \in \mathfrak{g}_{2} \mid\left[x, \mathfrak{g}_{-2}\right] \subseteq A_{0}\right\}$. By Lemma 1.48 (i), the set $\left\{\operatorname{ad}_{\mathfrak{g}_{-1}}[u, v] \mid u \in \mathfrak{g}_{-2}, v \in A_{2}\right\}$ is weakly closed in $\operatorname{End}\left(\mathfrak{g}_{-1}\right)$. Since

$$
0=(\operatorname{ad} u)^{2}(\operatorname{ad} v)^{2} \mathfrak{g}_{-1}=2(\operatorname{ad}[u, v])^{2} \mathfrak{g}_{-1}
$$

for all $u \in \mathfrak{g}_{-2}, v \in A_{2}$, Lemma 1.48 (ii) shows that $\left[\mathfrak{g}_{-2}, A_{2}\right]$ annihilates $\mathfrak{g}_{-1}$. Since $\mathfrak{g}$ is transitive, it must be that $\left[\mathfrak{g}_{-2}, A_{2}\right]=0$. But then $A_{2} \subseteq$ $\operatorname{Ann}_{\mathfrak{g}} S=0$. Since $\left[A_{0}, \mathfrak{g}_{2}\right] \subseteq A_{2}$, it follows that $A_{0}$ is an ideal of the Lie algebra $\mathfrak{g}_{-2} \oplus \mathfrak{g}_{0} \oplus \mathfrak{g}_{2}$.

Let $\mathcal{B}:=\overline{\mathfrak{g}}_{-1} \oplus \overline{\mathfrak{g}}_{0} \oplus \overline{\mathfrak{g}}_{1}$, where $\overline{\mathfrak{g}}_{i}=\left(\mathfrak{g}_{2 i}+A_{0}\right) / A_{0}$. The graded Lie algebra $\mathcal{B}$ is irreducible and transitive, but a priori it is not clear whether it is 1-transitive. Nevertheless, we will proceed as in part (a) of this proof.

Let $I=\overline{\mathfrak{g}}_{-1} \oplus\left[\overline{\mathfrak{g}}_{-1}, \overline{\mathfrak{g}}_{1}\right] \oplus \overline{\mathfrak{g}}_{1}$. Since any nonzero ideal of $\mathcal{B}$ contains $\overline{\mathfrak{g}}_{-1}$, we have $\mathfrak{Z}(I)=0$. Let $\bar{G}$ denote the connected component of the automorphism group of $I$ and $\bar{R}$ the solvable radical of the algebraic group $\bar{G}$. Let $\overline{\mathcal{R}}:=\operatorname{Lie}(\bar{R})$, a solvable subalgebra of $\operatorname{Der}(I)$. Since $\left(\operatorname{ad} \overline{\mathfrak{g}}_{ \pm 1}\right)^{3}(I)=0$, we have ad $I \subseteq \operatorname{Lie}(\bar{G})$. Hence ad $I$ is an ideal of $\operatorname{Lie}(\bar{G})$. Since $\overline{\mathcal{R}}$ is an ideal of $\operatorname{Lie}(\bar{G})$, we deduce that $[\overline{\mathcal{R}}, \operatorname{ad} I]$ is a solvable ideal of ad $I$. Let $\overline{\mathfrak{r}}$ denote the inverse image of $[\overline{\mathcal{R}}, I]$ under the isomorphism ad :I $\stackrel{\sim}{\longrightarrow}$ ad $I$.

Suppose $\overline{\mathcal{R}} \neq 0$. Then $[\overline{\mathcal{R}}$, ad $I] \neq 0$ and hence $\overline{\mathfrak{r}} \cap \overline{\mathfrak{g}}_{-1} \neq 0$ by the transitivity of $\mathcal{B}$. The irreducibility of $\overline{\mathfrak{g}}_{-1}$ shows that $A \operatorname{Ann}_{\overline{\mathfrak{g}}_{-1}} \overline{\mathfrak{g}}_{1}=0$. This, in turn, implies that $J:=\left[\overline{\mathfrak{r}} \cap \overline{\mathfrak{g}}_{-1}, \overline{\mathfrak{g}}_{1}\right]$ is a nonzero solvable ideal of $\left[\overline{\mathfrak{g}}_{-1}, \overline{\mathfrak{g}}_{1}\right]$. Lemma 1.71 says that $\overline{\mathfrak{g}}_{0}$ is classical reductive and $J \subseteq \mathfrak{Z}\left(\overline{\mathfrak{g}}_{0}\right)$. Arguing as in part (a) of this proof we reach a contradiction. Thus $\bar{G}$ is a semisimple algebraic group.

Since the center $Z(\bar{G})$ acts trivially on $\operatorname{Lie}(\bar{G})$, it also acts trivially on ad $I \subseteq \operatorname{Lie}(\bar{G})$. Since $\mathfrak{Z}(I)=0$, this yields that $\bar{G}$ is a group of adjoint type. The structure theory of algebraic $\mathbb{F}$-groups now shows that $\operatorname{Lie}(\bar{G})$ is classical reductive with $\mathfrak{Z}(\operatorname{Lie}(\bar{G}))=0$. Since any nonzero ideal of $I$ contains $\overline{\mathfrak{g}}_{-1}$ we can argue as in part (a) to deduce that $\bar{G}$ is a simple algebraic group. But then $I \cong \operatorname{ad} I$ is classical reductive, too. Moreover, its derived subalgebra $I^{(1)}$ is classical simple. Since $I \hookrightarrow \operatorname{Der}\left(I^{(1)}\right)$, the inequality $I \neq I^{(1)}$ would imply that $I^{(1)} \cong \mathfrak{p s l}_{k p}$ for some $k \in \mathbb{N}$ and $I \cong \mathfrak{p g l}_{k p} \cong \operatorname{Der}\left(\mathfrak{p s l}_{k p}\right)$. But then all nilpotent derivations of $I^{(1)}$ would be inner, forcing $\overline{\mathfrak{g}}_{1} \subset I^{(1)}$. This shows that $I$ is classical simple. Since $\mathcal{B}$ is irreducible and transitive, it embeds into $\operatorname{Der}(I)$. Therefore, either $\mathcal{B}=I$ or $\mathcal{B} \cong \mathfrak{p g l}_{k p}$ and $I=\mathcal{B}^{(1)}$. In any event, the simple algebraic group $\bar{G}$ has the property that $\operatorname{Lie}(\bar{G})=\operatorname{Der}(I)$.

(e) We now turn our attention to the Lie algebra $\widetilde{\mathcal{B}}:=\mathfrak{g}_{-2} \oplus \mathfrak{g}_{0} \oplus \mathfrak{g}_{2}$ and its ideal $\widetilde{I}:=\mathfrak{g}_{-2} \oplus\left[\mathfrak{g}_{-2}, \mathfrak{g}_{2}\right] \oplus \mathfrak{g}_{2}$. Let $\varphi: \widetilde{\mathcal{B}} \rightarrow \mathcal{B}=\widetilde{\mathcal{B}} / A_{0}$ denote the canonical 
homomorphism. Since $A_{0}$ annihilates $\mathfrak{g}_{2}$ and $\mathfrak{g}_{-2}$, it commutes with $\widetilde{I}$. So $\widetilde{I} \cap A_{0}$ is an abelian ideal of $\mathfrak{g}_{0}$. Since $\mathfrak{g}_{0}$ is classical reductive, $\widetilde{I} \cap A_{0} \subseteq \mathfrak{Z}\left(\mathfrak{g}_{0}\right)$. Since $\mathfrak{Z}\left(\mathfrak{g}_{0}\right)$ acts faithfully on $\mathfrak{g}_{-2}=\left[\mathfrak{g}_{-1}, \mathfrak{g}_{-1}\right]$, we get $\widetilde{I} \cap A_{0}=0$. Therefore, $\widetilde{I} \cong \varphi(\widetilde{I})=I$, and we can identify $\operatorname{Der}(\widetilde{I})$ with $\operatorname{Lie}(\bar{G})$. Since $\mathfrak{Z}(\operatorname{Der}(\widetilde{I}))=0$, the natural $p$ th power map on $\operatorname{Der}(\widetilde{I})$ then gets identified with the canonical $(\operatorname{Ad} \bar{G})$-equivariant $p$ th power map on $\operatorname{Lie}(\bar{G})$.

The adjoint action of $\widetilde{\mathcal{B}}$ on $\widetilde{I}$ induces a natural Lie algebra homomorphism $\psi: \widetilde{\mathcal{B}} \rightarrow \operatorname{Der}(\widetilde{I})=\operatorname{Lie}(\bar{G})$. Since $\mathfrak{g}_{0}$ is classical reductive, it contains a diagonalizable Cartan subalgebra, $\mathfrak{h}$ say. We denote by $\mathcal{H}$ the image of $\mathfrak{h}$ under $\psi$. Since $\mathfrak{g}_{-1}$ is an irreducible $\mathfrak{g}_{0}$-module, $\mathfrak{h}$ acts diagonalizably on $\mathfrak{g}_{-1}$; see Lemma 1.67. Therefore, $\mathfrak{h}$ acts diagonalizably on $\mathfrak{g}_{-1} \hookrightarrow \operatorname{Hom}\left(\mathfrak{g}_{-1}, \mathfrak{g}_{0}\right)$. Since $\mathfrak{g}$ is generated by its local part, $\mathfrak{h}$ acts diagonalizably on $\mathfrak{g}$. As a consequence, $\mathcal{H}$ is a diagonalizable subalgebra of $\operatorname{Lie}(\bar{G})$, i.e., consists of pairwise commuting $[p]$-semisimple elements of $\operatorname{Lie}(\bar{G})$. Let $\mathcal{C}$ (respectively, $\mathcal{C}^{\prime}$ ) denote the centralizer of $\mathcal{H}$ in $\operatorname{Der}(\widetilde{I})$ (respectively, in ad $\widetilde{I}$ ). Clearly, $\mathcal{C}^{\prime}$ is an ideal of codimension $\leq 1$ in $\mathcal{C}$. It is easy to see that $\mathcal{C}^{\prime}$ is isomorphic to the centralizer of $\mathfrak{h}$ in $\widetilde{I}$. The group $\bar{G}$ acts on its Lie algebra $\operatorname{Lie}(\bar{G})=\operatorname{Der}(\widetilde{I})$ via the adjoint representation $\mathrm{Ad}$, and we have $(\operatorname{Ad} g)(D)=g \circ D \circ g^{-1}$ for all $g \in \bar{G}$ and $D \in \operatorname{Der}(\widetilde{I})$. Let

$$
Z_{\bar{G}}(\mathcal{H}):=\{x \in \bar{G} \mid(\operatorname{Ad} g)(x)=x \text { for all } x \in \mathcal{H}\},
$$

the centralizer of $\mathcal{H}$ in $\bar{G}$. Thanks to $\left[\right.$ Bo, Sec. 9.1] we have $\mathcal{C}=\operatorname{Lie}\left(Z_{\bar{G}}(\mathcal{H})\right)$, while [Bo, Sec. 13.19] says that the connected component of $Z_{\bar{G}}(\mathcal{H})$ is reductive. As a consequence, $\mathcal{C}$ is isomorphic to a Lie algebra of reductive group. Let

$$
\mathfrak{c}^{\prime}:=\mathfrak{g}_{-2}^{\mathfrak{h}} \oplus\left(\mathfrak{h} \cap\left[\mathfrak{g}_{-2}, \mathfrak{g}_{2}\right]\right) \oplus \mathfrak{g}_{2}^{\mathfrak{h}}
$$

where $\mathfrak{g}_{ \pm 2}^{\mathfrak{h}}=\left\{x \in \mathfrak{g}_{ \pm 2} \mid[x, \mathfrak{h}]=0\right\}$. Then $\mathfrak{c}^{\prime} \cong \mathcal{C}^{\prime}$ by our earlier remarks. The set

$$
\left\{\left.(\operatorname{ad} x)\right|_{\mathfrak{c}^{\prime}} \mid x \in \mathfrak{g}_{-2}^{\mathfrak{h}} \cup\left(\mathfrak{h} \cap\left[\mathfrak{g}_{-2}, \mathfrak{g}_{2}\right]\right) \cup \mathfrak{g}_{2}^{\mathfrak{h}}\right\}
$$

is weakly closed and consists of nilpotent endomorphisms of $\mathfrak{c}^{\prime}$. Applying $[\mathbf{J}$, p. 33] (compare Lemma 1.48 (ii)), we now deduce that the Lie algebra $\mathcal{C}^{\prime}$ is nilpotent. Since $\mathrm{C}^{\prime}$ is an ideal of codimension $\leq 1$ in $\mathcal{C}$, the Lie algebra $\mathrm{C}=$ $\operatorname{Lie}\left(Z_{\bar{G}}(\mathcal{H})\right)$ is solvable. Since the group $Z_{\bar{G}}(\mathcal{H})$ is reductive, the connected component $Z_{\bar{G}}(\mathcal{H})^{\circ}$ must be a torus. It follows that $\mathcal{C}$ is abelian and consists of $[p]$-semisimple elements of $\operatorname{Lie}(\bar{G})$. This, in turn, implies that $\mathfrak{c}^{\prime}$ is a diagonalizable subalgebra of $\widetilde{I}$, yielding $\mathfrak{g}_{ \pm 2}^{\mathfrak{h}}=0$.

Given $t \in \mathbb{F}^{\times}$define $\lambda(t) \in$ Aut $\widetilde{I}$ by setting $\lambda(t)(x)=t^{i} x$ for all $x \in$ $\mathfrak{g}_{i} \cap \widetilde{I}$. Since $\lambda\left(\mathbb{F}^{\times}\right)$is a one-dimensional torus, $\lambda\left(\mathbb{F}^{\times}\right)$is contained in $\bar{G}$. Since $\mathcal{H}$ preserves the graded components of $\widetilde{I}$, we have $\lambda\left(\mathbb{F}^{\times}\right) \subseteq Z_{\bar{G}}(\mathcal{H})^{\circ}$. Also,

$$
\operatorname{Lie}(\bar{G})=\operatorname{Lie}(\bar{G})_{-2} \oplus \operatorname{Lie}(\bar{G})_{0} \oplus \operatorname{Lie}(\bar{G})_{2}
$$


where $\operatorname{Lie}(\bar{G})_{i}=\left\{x \in \operatorname{Lie}(\bar{G}) \mid(\operatorname{Ad} \lambda(t)) x=t^{i} x\right.$ for all $\left.t \in \mathbb{F}^{\times}\right\}$. Let $Z_{\bar{G}}(\lambda)$ denote the centralizer of $\lambda\left(\mathbb{F}^{\times}\right)$in $\bar{G}$. By [Sp, Sec. 8.4], there exists a parabolic subgroup $P$ of $\bar{G}$ with Levy decomposition $P=Z_{\bar{G}}(\lambda) R_{u}(P)$ (where $R_{u}(P)$ is the unipotent radical of $P$ ) such that $\operatorname{Lie}\left(R_{u}(P)\right)=\operatorname{Lie}(\bar{G})_{2}$. Let $T$ be a maximal torus of $\bar{G}$ containing $Z_{\bar{G}}(\mathcal{H})^{\circ}$. Then $T \subset Z_{\bar{G}}(\lambda)$ and

$$
\mathcal{H} \subseteq \mathcal{C}=\operatorname{Lie}\left(Z_{\bar{G}}(\mathcal{H})\right)^{\circ} \subseteq \operatorname{Lie}(T) .
$$

Let $B$ be a Borel subgroup of $P$ containing $T$. Then $B$ is a Borel subgroup of $\bar{G}$ and $R_{u}(P)$ is a normal subgroup of $B$. Therefore, $\operatorname{Lie}\left(R_{u}(P)\right)=\operatorname{Lie}(\bar{G})_{2}$ is $(\operatorname{Ad} B)$-stable. But then $\operatorname{ad} \mathfrak{g}_{2}=(\operatorname{ad} \widetilde{I}) \cap \operatorname{Lie}(\bar{G})_{2}$ is $(\operatorname{Ad} B)$-stable as well. It follows that $\mathfrak{g}_{2}$ contains a nonzero element fixed by the derived subgroup $(B, B)$ of $B$, say $e$.

Since $\widetilde{I}$ is an irreducible $\bar{G}$-module, the subspace of $(B, B)$-invariants in $\widetilde{I}$ is one-dimensional, hence equals $\mathbb{F} e$. Since $B=T(B, B)$, the line $\mathbb{F}$ ad $e$ is a root space for $\operatorname{Ad} T$. Let $\Psi$ denote the root system of $\bar{G}$ relative to $T$ and $\Psi_{+}(B)$ the positive system of $\Psi$ corresponding to $B$. Note that all root spaces of $\operatorname{Lie}(\bar{G})$ lie in ad $\widetilde{I}$ (for $\operatorname{Lie}(\bar{G}) / \operatorname{ad} \widetilde{I}$ is a trivial $\bar{G}$-module). Let $\gamma$ denote the root of ad $e$. It is immediate from the above discussion that $\gamma$ is the highest root in $\Psi_{+}(B)$. As $\gamma(\lambda(t))=t^{2}$ for all $t \in \mathbb{F}^{\times}$, there is a nonzero $f \in \mathfrak{g}_{-2}$ such that ad $f$ is a root vector corresponding to $-\gamma$. Since $\mathcal{H} \subseteq \operatorname{Lie}(T)$, there is a linear function $\eta$ on $\mathfrak{h}$ such that

$$
[h, e]=\eta(h) e, \quad[h, f]=-\eta(h) f \quad(\forall h \in \mathfrak{h})
$$

(to be more precise, $\eta(h)=\left((\mathrm{d} \gamma)_{e} \circ \psi\right)(h)$ where $(\mathrm{d} \gamma)_{e}$ is the differential of the morphism $\gamma: T \rightarrow \mathbb{F}^{\times}$at the identity element of $T$ ). Since ad $e$ and ad $f$ belong to the Lie algebra of a semisimple subgroup of rank one in $\bar{G}$, they generate an $\mathfrak{s l}_{2}$-triple in $\operatorname{Lie}(\bar{G})$. Let $h=[e, f]$. No generality is be lost by assuming that $\{e, h, f\}$ is a standard basis of $\mathfrak{s}:=\mathbb{F} f \oplus \mathbb{F} h \oplus \mathbb{F} e \cong \mathfrak{s l}_{2}$. Since all root spaces of $\operatorname{Ad} T$ are one-dimensional and $2 \gamma+\alpha>\gamma$ for all $\alpha \in \Psi \backslash\{-\gamma\}$, we have the equalities $(\operatorname{ad} e)^{2}(\widetilde{I})=\mathbb{F} e$ and $(\operatorname{ad} f)^{2}(\widetilde{I})=\mathbb{F} f$.

(f) Let $E, H$, and $F$ denote the adjoint mappings on $S=\bigoplus_{i \geq 0}\left(\operatorname{ad} \mathfrak{g}_{1}\right)^{i} \mathfrak{g}_{-q}$ $(q=2)$ corresponding to $e, h$, and $f$, respectively. Then $S$ is an $\mathfrak{s}$-module with $E^{3}=F^{3}=0$ so that

$$
E^{p-1}(H+1)=0=(H+1) F^{p-1} .
$$

Therefore, $[\mathbf{S}$, Sec. 5.2] implies that the $\mathfrak{s}$-module $S$ is completely reducible with composition factors $L(0), L(1), L(2)$, where $L(k)=L\left(k \varpi_{1}\right)$. In particular, all eigenvalues of $H$ are in $\mathbb{F}_{p}$. Let $V_{k}$ denote the eigenspace of $H$ in $S$ corresponding to $k \in \mathbb{F}_{p}$. Since $E^{2}(S)=(\operatorname{ad} e)^{2}(\widetilde{I})=\mathbb{F} e$ by part (e), and $\operatorname{dim} V_{2}=\operatorname{dim} E^{2}(S)$ equals the multiplicity of $L(2)$ in $S$, we have $V_{2}=\mathbb{F} e$. Similarly, $V_{-2}=\mathbb{F} f$. As $p=5$, we have $E\left(V_{1}\right) \subseteq V_{-2}$ and $F\left(V_{-1}\right) \subseteq V_{2}$. The equalities $E^{3}=F^{3}=0$ now yield $\left[V_{1}, V_{2}\right]=\left[V_{-1}, V_{-2}\right]=0$. But then the decomposition

$$
S=V_{-2} \oplus V_{-1} \oplus V_{0} \oplus V_{1} \oplus V_{2}
$$


is a $\mathbb{Z}$-grading of $S$. Since $h \in \mathfrak{h}$, all graded components $V_{i}$ are $\mathfrak{h}$-stable.

As explained in part (e), the subalgebra $\mathfrak{h}$ acts diagonalizably on $S$. Thus, any $\mathfrak{h}$-stable subspace $M$ of $S$ decomposes as $M=\bigoplus_{\alpha \in \mathfrak{h}^{*}} M^{\alpha}$ where $M^{\alpha}=\{x \in M \mid[h, x]=\alpha(h) x$ for all $h \in \mathfrak{h}\}$. By our remarks in part (e), $V_{2}=V_{2}^{\eta}$ and $V_{-2}=V_{-2}^{-\eta}$.

(g) The graded component $V_{1}$ is spanned by the set $X:=\bigcup_{i \in \mathbb{Z}, \alpha(h)=1} \mathfrak{g}_{i}^{\alpha}$. We claim that $(\operatorname{ad} x)^{4}=0$ for all $x \in \mathcal{X}$. If $x \in \mathfrak{g}_{i}^{\alpha}$ and $\alpha \neq \eta / 2$, then

$$
(\operatorname{ad} x)^{4}(S)=(\operatorname{ad} x)^{4}\left(V_{-2}^{-\eta}\right) \subseteq V_{2}^{-\eta-\alpha}=0,
$$

so our claim is valid in this case. Assume for a contradiction that there is a $y \in \mathfrak{g}_{s}^{\eta / 2}$, for some $s \in \mathbb{Z}$, such that $(\operatorname{ad} y)^{4} \neq 0$. Notice that $\mathfrak{g}^{-\eta}=\mathfrak{g}_{-2}^{-\eta}=\mathbb{F} f$ and $\mathfrak{g}^{\eta}=\mathfrak{g}_{2}^{\eta}=\mathbb{F} e$. Since $y \in V_{1}$, we have $(\operatorname{ad} y)^{4}(S)=(\operatorname{ad} y)^{4}(\mathbb{F} f) \subseteq \mathfrak{g}_{2}$. As $f \in \mathfrak{g}_{-2}$, it must be that $s=1$. We now consider

$$
\mathfrak{g}(\eta):=\mathfrak{g}_{-2}^{-\eta} \oplus \mathfrak{g}_{-1}^{-\eta / 2} \oplus \mathfrak{h} \oplus \mathfrak{g}_{1}^{\eta / 2} \oplus \mathfrak{g}_{2}^{\eta},
$$

a graded subalgebra of $\mathfrak{g}$ (one should take into account that $\left[\mathfrak{g}_{-1}^{\eta / 2}, \mathfrak{g}_{1}^{-\eta / 2}\right] \subseteq \mathfrak{h}$ as $\mathfrak{h}$ is self-centralizing in $\left.\mathfrak{g}_{0}\right)$. Let $\mathfrak{h}_{0}$ denote the kernel of $\eta$, a subspace in $\mathfrak{Z}(\mathfrak{g}(\eta))$. Consider the pairing

$$
\xi: \mathfrak{g}_{-1}^{-\eta / 2} \times \mathfrak{g}_{1}^{\eta / 2} \longrightarrow \mathfrak{h} / \mathfrak{h}_{0}, \quad(x, y) \longmapsto[x, y]\left(\bmod \mathfrak{h}_{0}\right) .
$$

Let $W_{i}$ denote the orthogonal subspace to $\xi$ in $\mathfrak{g}_{i}^{i \eta / 2}, i=-1,1$. By our assumption, $y \notin W_{1}$. If $W_{1}$ had codimension $\geq 2$ in $\mathfrak{g}_{1}^{\eta / 2}$, we would be able to find $a_{1}, a_{2} \in \mathfrak{g}_{1}^{\eta / 2}$ and $b_{1}, b_{2} \in \mathfrak{g}_{-1}^{-\eta / 2}$ such that $\xi\left(a_{i}, b_{j}\right)=\delta_{i j}$ for all $i, j \in\{1,2\}$. But then the images of $a_{1}, a_{2}, b_{1}, b_{2}$ and $h$ would generate an infinite-dimensional subalgebra of $\mathfrak{g}(\eta) / \mathfrak{h}_{0}$; see Theorem 3.7. Therefore, $W_{i}$ has codimension one in $\mathfrak{g}_{i}, i=-1,1$. Since $E\left(\mathfrak{g}_{1}\right)=F\left(\mathfrak{g}_{-1}\right)=0$ and $\eta(h)=2$, the map $E$ induces a linear isomorphism between $\mathfrak{g}_{-1}^{-\eta / 2}$ and $\mathfrak{g}_{1}^{\eta / 2}$. On the other hand, our assumptions on $y$ and $s$ imply that $e \in\left[\mathfrak{g}_{1}^{\eta / 2}, \mathfrak{g}_{1}^{\eta / 2}\right]$. Hence

$$
E\left(W_{-1}\right) \subseteq\left[\mathfrak{g}_{1}^{\eta / 2},\left[\mathfrak{g}_{1}^{\eta / 2}, W_{-1}\right]\right] \subseteq\left[\mathfrak{g}_{1}^{\eta / 2}, \mathfrak{h}_{0}\right]=0,
$$

yielding $W_{-1}=0$. As a result, $\operatorname{dim} \mathfrak{g}_{1}^{\eta / 2}=1$, that is $\mathfrak{g}_{1}^{\eta / 2}=\mathbb{F} y$. But then $(\operatorname{ad} y)^{4}(f)=0$, a contradiction. The claim follows.

(h) We now follow $[\mathbf{P 1}$, Sec. 3] to show that for all $x \in X$ and $t \in \mathbb{F}$ the linear map $\exp (\operatorname{tad} x)$ belongs to $G$, the connected component of the automorphism group $\operatorname{Aut}(S)$ of $S$. Let $X=\operatorname{ad} x$. Since $X^{4}=0$ by part (g), this amounts to proving that

$$
\left[X^{2}(u), X^{3}(v)\right]=0 \quad(\forall u, v \in S) .
$$

Let $u \in V_{i}$ and $v \in V_{j}$ where $-2 \leq i, j \leq 2$ (we can view $i$ and $j$ as integers here). Since $X^{4}([X(u), v])=0$ and $p=5$, it follows that

$$
\left[X^{3}(u), X^{2}(v)\right]=\left[X^{2}(u), X^{3}(v)\right]=\frac{1}{2} X\left(\left[X^{2}(u), X^{2}(v)\right]\right) .
$$


Note that $X^{3}$ annihilates $V_{0} \oplus V_{1} \oplus V_{2}$. So if $\left[X^{2}(u), X^{3}(v)\right] \neq 0$, then $u, v \in V_{-1} \cup V_{-2}$. Since $\operatorname{dim} V_{-2}=1$, we also have $\{u, v\} \not \subset V_{-2}$, while the equality $\left[V_{1}, V_{2}\right]=0$ implies $\{u, v\} \not \subset V_{-1}$. In view of what is displayed above, we may thus assume that $u=f$ and $v \in V_{-1}$. But then $X^{3}(v)=\mu e$ for some $\mu \in \mathbb{F}$, and

$$
\left[X^{2}(u), X^{3}(v)\right]=\mu[[x,[x, f]], e]=-\mu[x,[x, h]]=0 .
$$

Thus, we have shown that $\exp t X \in G$ for all $x \in X$ and $t \in \mathbb{F}$. Since $X$ spans $V_{1}$, it follows that ad $V_{1} \subset \mathcal{L}=\operatorname{Lie}(G)$. Since $E, F \in \mathcal{L}$ too, and ad $V_{-1}=\left[F\right.$, ad $\left.V_{1}\right]$, we derive that $\mathcal{L}$ contains a nonzero ideal of $\mathfrak{g}$. But then ad $S \subseteq \mathcal{L}$. By part (a) of this proof the result follows.

\subsection{Algebras with an unbalanced grading}

The previous section dealt with balanced gradings, so what remains to be considered are the Lie algebras $\mathfrak{g}$ with $\mathfrak{g}_{1} \cong\left(\mathfrak{g}_{-1}\right)^{*}$ as $\mathfrak{g}_{0}$-modules but with an unbalanced grading. We will address such algebras in this section and complete the proof of our final (main) result on the contragredient case:

TheOREM 3.22. Assume $\mathfrak{g}=\bigoplus_{j=-q}^{r} \mathfrak{g}_{j}$ is a graded Lie algebra over an algebraically closed field $\mathbb{F}$ of characteristic $p>3$. Suppose $\mathfrak{g}_{0}$ is classical reductive with $\mathfrak{g}_{0}^{(1)} \neq 0, \mathfrak{g}$ is transitive (1.2) and 1 -transitive (1.3), and $\mathfrak{g}_{-1}$ is an irreducible restricted $\mathfrak{g}_{0}^{(1)}$-module. Assume further that $\mathfrak{g}_{1}$ is an irreducible $\mathfrak{g}_{0}$-module generated by a $\mathfrak{b}^{-}$-primitive vector of weight $-\Lambda$. Then either $\mathfrak{g}$ is a classical Lie algebra with one of its standard gradings (see Section 2.4), or else $p=5$ and $\mathfrak{g}$ is a Melikyan algebra. In the latter case, the grading of $\mathfrak{g}$ is the natural grading if $r \geq q$ and is opposite to the natural grading if $r<q$.

Proof. We assume that

(i) $\mathfrak{g}_{0}=\mathfrak{g}_{0}^{[1]} \oplus \cdots \oplus \mathfrak{g}_{0}^{[\ell]}$ is a decomposition of $\mathfrak{g}_{0}$ into ideals $\mathfrak{g}_{0}^{[i]}$ which are classical simple, $\mathfrak{s l}_{n}, \mathfrak{g l}_{n}, \mathfrak{p g l}_{n}$ where $p \mid n$, or one-dimensional;

(ii) $\mathfrak{t}$ is a fixed maximal toral subalgebra in $\mathfrak{g}_{0}$ and $\mathfrak{t}^{[i]}=\mathfrak{t} \cap \mathfrak{g}_{0}^{[i]}$;

(iii) $\Phi=\Phi^{[1]} \cup \cdots \cup \Phi^{[\ell]}$, where $\Phi^{[i]}$ is the root system of $\mathfrak{g}_{0}^{[i]}$ with respect $\mathfrak{t}^{[i]} ; \quad \Delta=\left\{\alpha_{1}, \ldots, \alpha_{m}\right\}$ is a system of simple roots in $\Phi$; and $\Phi^{+}$ and $\Phi^{-}$are the positive and negative roots respectively relative to $\Delta$.

(iv) for $\alpha \in \Phi$, the root vector $e_{\alpha}$ spans the root space $\mathfrak{g}_{0}^{\alpha}$, and the triple of vectors $\left(e_{\alpha}, e_{-\alpha}, h_{\alpha}\right)$, where $h_{\alpha} \in \mathfrak{t}$, form a standard basis for a copy of $\mathfrak{s l}_{2}$ :

$$
\left[e_{\alpha}, e_{-\alpha}\right]=h_{\alpha}, \quad\left[h_{\alpha}, e_{\alpha}\right]=2 e_{\alpha}, \quad\left[h_{\alpha}, e_{-\alpha}\right]=-2 e_{-\alpha} .
$$

(v) $\mathfrak{n}^{+}=\bigoplus_{\alpha \in \Phi^{+}} \mathfrak{g}_{0}^{\alpha}, \mathfrak{n}^{-}=\bigoplus_{\alpha \in \Phi^{-}} \mathfrak{g}_{0}^{\alpha}, \mathfrak{b}^{+}=\mathfrak{t} \oplus \mathfrak{n}^{+}$, and $\mathfrak{b}^{-}=\mathfrak{t} \oplus \mathfrak{n}^{-}$. 
For $j \geq 0$, the $\mathfrak{g}_{0}$-module $\mathfrak{g}_{j+1}$ embeds into $\operatorname{Hom}\left(\mathfrak{g}_{-1}, \mathfrak{g}_{j}\right)$ by transitivity (1.2). For $j \leq 0$, the $\mathfrak{g}_{0}$-module $\mathfrak{g}_{j-1}$ embeds into $\operatorname{Hom}\left(\mathfrak{g}_{1}, \mathfrak{g}_{j}\right)$ by 1-transitivity (1.3). Easy induction on $|j|$ now shows that all homogeneous pieces $\mathfrak{g}_{j}$ are restricted $\mathfrak{g}_{0}^{(1)}$-modules .

Let $e^{-\Lambda}$ be a nonzero $\mathfrak{b}^{-}$-primitive vector of weight $-\Lambda$ in $\mathfrak{g}_{1}$, and let $\widetilde{\mathfrak{g}}$ be the graded subalgebra of $\mathfrak{g}$ generated by $\mathfrak{g}_{0}, f^{\Lambda}$, and $e^{-\Lambda}$. Set $\widetilde{\mathfrak{g}}_{i}=\widetilde{\mathfrak{g}} \cap \mathfrak{g}_{i}$. As $\widetilde{\mathfrak{g}}_{ \pm 1}=\mathfrak{g}_{ \pm 1}$ by our initial assumption, the graded Lie algebra $\widetilde{\mathfrak{g}}$ is transitive and 1-transitive. It follows that the Weisfeiler radical $\mathcal{M}(\widetilde{\mathfrak{g}})$ of $\widetilde{\mathfrak{g}}$ is zero. Since our assumption on $\mathfrak{g}_{1}$ implies that $\mathfrak{g}_{1} \cong \mathfrak{g}_{-1}^{*}$ as $\mathfrak{g}_{0}^{(1)}$-modules, $\widetilde{\mathfrak{g}}$ satisfies the assumptions of Theorem 3.21. By that result, $\widetilde{\mathfrak{g}}$ is a classical Lie algebra with a standard grading (where $e^{-\Lambda}$ has degree $1, f^{\Lambda}$ degree -1 , and elements of $\mathfrak{g}_{0}$ have degree 0$)$.

Suppose now that $\widetilde{\mathfrak{g}}_{t} \neq \mathfrak{g}_{t}$ for some $t \geq 2$, but $\widetilde{\mathfrak{g}}_{i}=\mathfrak{g}_{i}$ for all $1 \leq i<t$. Let $x_{\beta} \in \mathfrak{g}_{t}$ be such that $x_{\beta}+\widetilde{\mathfrak{g}}_{t}$ is a $\mathfrak{b}^{-}$-primitive vector of weight $\beta$ for the $\mathfrak{g}_{0}$-module $\mathfrak{g}_{t} / \widetilde{\mathfrak{g}}_{t}$. Then $\left[f^{\Lambda}, x_{\beta}\right] \in \widetilde{\mathfrak{g}}_{t-1}$ and $\left[f_{j}, x_{\beta}\right] \in \widetilde{\mathfrak{g}}_{t}$ for all $f_{j}:=e_{-\alpha_{j}}$ $(j=1, \ldots, m)$. Set $f_{0}:=f^{\Lambda}$ and $\alpha_{0}:=-\Lambda$. The elements $f_{0}, f_{1}, \ldots, f_{m}$ generate a maximal nilpotent subalgebra $\widetilde{\mathfrak{n}}^{-} \subset \widetilde{\mathfrak{g}}$. Since $\widetilde{\mathfrak{n}}^{-} \supset \mathfrak{g}-1$ and $\mathfrak{g}$ is transitive, there exists an $l \in\{0,1 \ldots, m\}$ for which $\left[f_{l}, x_{\beta}\right] \neq 0$. Thus, $\beta-\alpha_{l} \in \Phi(\widetilde{\mathfrak{g}})$, the set of roots of $\widetilde{\mathfrak{g}}$.

Let us suppose first that $\beta$ is a root of $\widetilde{\mathfrak{g}}$ and that $\left[f_{l}, e_{\beta}\right] \neq 0$. Write $\beta=\sum_{i=0}^{m} b_{i} \alpha_{i}$. Then $b_{0}=\operatorname{deg}\left(e_{\beta}\right)=\operatorname{deg}\left(x_{\beta}\right)=t \geq 2$. Since twice a root is never a root in a classical Lie algebra ([S, Lem. II.2.5]), it follows that $\beta$ has height ht $(\beta)=\sum_{i=0}^{m} b_{i} \geq 3$.

Since root spaces of a classical Lie algebra are one-dimensional (Lemma II.2.6 of $[\mathbf{S}])$, there is some scalar $a \in \mathbb{F}^{\times}$for which $\left[f_{l}, x_{\beta}-a e_{\beta}\right]=0$. If $\beta-\alpha_{k} \notin \Phi(\widetilde{\mathfrak{g}})$ for all $k \neq l$, then $\left[f_{k}, x_{\beta}-a e_{\beta}\right]=0$ for all such $k$. But then $\left[\widetilde{\mathfrak{n}}^{-}, x_{\beta}-a e_{\beta}\right]=0$, contradicting the transitivity of $\mathfrak{g}$. Thus, there must exist a $k \neq l$ such that $\beta-\alpha_{k}$ belongs to $\Phi(\widetilde{\mathfrak{g}})$.

Suppose $\Phi(\widetilde{\mathfrak{g}})$ has rank two. Then $\widetilde{\Delta}:=\left\{\alpha_{k}, \alpha_{l}\right\}$ is a basis of $\Phi(\widetilde{\mathfrak{g}})$ and $\beta$ is a root of height $\geq 3$ in $\Phi(\widetilde{\mathfrak{g}})$ with the property that $\beta-\alpha \in \Phi(\widetilde{\mathfrak{g}})$ for all $\alpha \in \widetilde{\Delta}$. Since $\mathbb{Z} \gamma \cap \Phi(\widetilde{\mathfrak{g}})= \pm \gamma$ for all $\gamma \in \Phi(\widetilde{\mathfrak{g}})$, this is impossible. Therefore, the rank of the root system $\Phi(\widetilde{\mathfrak{g}})$ is $\geq 3$.

Set

$$
\widetilde{\Delta}(\beta):=\left\{\alpha_{i} \mid \beta-\alpha_{i} \in \Phi(\widetilde{\mathfrak{g}})\right\} .
$$

If all of the roots in $\Phi(\widetilde{\mathfrak{g}})$ have the same length (i.e., if the root system is simply laced), and if $\alpha_{i}$ and $\alpha_{j}$ are distinct simple roots in $\widetilde{\Delta}(\beta)$, then $\left(\alpha_{i}, \alpha_{j}\right)=0$. To see this note that when all roots have equal length, then root strings through linearly independent roots have length at most 2 . Thus, since $\alpha_{i} \in \widetilde{\Delta}(\beta)$, we have 


$$
\frac{2\left(\beta, \alpha_{i}\right)}{\left(\alpha_{i}, \alpha_{i}\right)}=1
$$

and similarly for $\alpha_{j}$. If $\left(\alpha_{i}, \alpha_{j}\right) \neq 0$, then $\alpha_{i}+\alpha_{j}$ is a root, and

$$
\frac{2\left(\beta, \alpha_{i}+\alpha_{j}\right)}{\left(\alpha_{i}+\alpha_{j}, \alpha_{i}+\alpha_{j}\right)}=\frac{2\left(\beta, \alpha_{i}\right)}{\left(\alpha_{i}, \alpha_{i}\right)}+\frac{2\left(\beta, \alpha_{j}\right)}{\left(\alpha_{j}, \alpha_{j}\right)}=2 .
$$

This forces $\beta$ to equal $\alpha_{i}+\alpha_{j}$, which contradicts the fact that ht $\beta \geq 3$. Therefore, when $\Phi(\mathfrak{g})$ is simply laced, we must have $\left(\alpha_{i}, \alpha_{j}\right)=0$ for all $\alpha_{i}, \alpha_{j} \in \widetilde{\Delta}(\beta)$.

We have argued earlier that there exists a $k \neq l$ so that $\left[f_{k}, x_{\beta}-a e_{\beta}\right] \neq 0$. Since $\alpha_{k}$ and $\alpha_{l}$ both belong to $\widetilde{\Delta}(\beta)$, we must have $\left(\alpha_{k}, \alpha_{l}\right)=0$ in the simply laced case, and $\left[f_{k}, f_{l}\right]=0$. But then

$$
\left(\beta-\alpha_{k}, \alpha_{l}\right)=\left(\beta, \alpha_{l}\right)>0,
$$

so that $\left[f_{l},\left[f_{k}, x_{\beta}-a e_{\beta}\right]\right] \neq 0$. On the other hand,

$$
\left[f_{l},\left[f_{k}, x_{\beta}-a e_{\beta}\right]\right]=\left[\left[f_{l}, f_{k}\right], x_{\beta}-a e_{\beta}\right]+\left[f_{k},\left[f_{l}, x_{\beta}-a e_{\beta}\right]\right]=0 .
$$

This contradiction shows that all roots cannot have equal length, so it must be that $\Phi(\widetilde{\mathfrak{g}})$ is of type $\mathrm{B}_{m+1}, \mathrm{C}_{m+1}$, or $\mathrm{F}_{4}$ with $m \geq 2$, since the rank is at least 3 .

Suppose first that $\Phi(\widetilde{\mathfrak{g}})$ is of type $\mathrm{B}_{m+1}$. Let $\dot{\alpha}_{0}, \dot{\alpha}_{1}, \ldots, \dot{\alpha}_{m}$ denote the labeling of the simple roots of $\mathrm{B}_{m+1}$ compatible with that in [Bou1, Planche II] so that the highest root is

$$
\dot{\alpha}_{0}+2 \dot{\alpha}_{1}+\cdots+2 \dot{\alpha}_{m-1}+2 \dot{\alpha}_{m}
$$

Since the coefficient of $\alpha_{0}$ in the expression for $\beta$ is $b_{0} \geq 2$, it must be that $\alpha_{0}=\dot{\alpha}_{s}$ for some $1 \leq s \leq m$. Thus, $\beta$ must be of the form

$$
\sum_{j \leq i<l} \dot{\alpha}_{i}+2 \sum_{l \leq n \leq m} \dot{\alpha}_{n}
$$

for $0 \leq j<l \leq m-1$, and $\widetilde{\Delta}(\beta)=\left\{\dot{\alpha}_{j}, \dot{\alpha}_{l}\right\}$.

Likewise, if $\Phi(\widetilde{\mathfrak{g}})$ is of type $\mathrm{C}_{m+1}$, then the highest root is

$$
2 \dot{\alpha}_{0}+2 \dot{\alpha}_{1}+\cdots+2 \dot{\alpha}_{m-1}+\dot{\alpha}_{m} .
$$

The root $\beta$ must have the form

$$
\sum_{j \leq i<l} \dot{\alpha}_{i}+2\left(\sum_{l \leq n<m} \dot{\alpha}_{n}\right)+\dot{\alpha}_{m} \quad \text { or } \quad 2\left(\sum_{j \leq l<m} \dot{\alpha}_{l}\right)+\dot{\alpha}_{m}
$$


The second of these possibilities cannot happen, since $\widetilde{\Delta}(\beta)=\left\{\dot{\alpha}_{j}\right\}$ in that case. For the first, $\widetilde{\Delta}(\beta)=\left\{\dot{\alpha}_{j}, \dot{\alpha}_{l}\right\}$ just as in the $\mathrm{B}_{m+1}$-case.

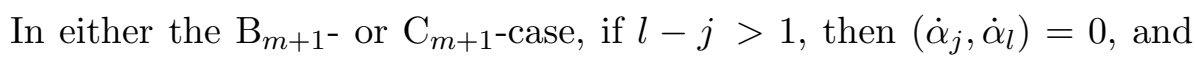
we can use an argument as in (3.23) to arrive at a contradiction. If instead $l-j=1$, then $\widetilde{\Delta}(\beta)=\left\{\dot{\alpha}_{j}, \dot{\alpha}_{j+1}\right\}$. Since $j \leq m-2$, the Cartan matrix entry $A_{j, j+1}=-1$, so that the length of the $\dot{\alpha}_{j+1}$-string through $\dot{\alpha}_{j}$ is 1 . If $\dot{\alpha}_{j}=\alpha_{k}$, then set $\dot{f}_{j}=f_{k}$, and adopt a similar convention for $\dot{\alpha}_{j+1}$.

Consequently $\left[\dot{f}_{j+1}\left[\dot{f}_{j+1}, \dot{f}_{j}\right]\right]=0$, and both $\beta-\dot{\alpha}_{j}-\dot{\alpha}_{j+1}$ and $\beta-$ $\dot{\alpha}_{j}-2 \dot{\alpha}_{j+1}$ are roots of $\widetilde{\mathfrak{g}}$. As root spaces of a classical Lie algebra are onedimensional, we can choose an appropriate $c \in \mathbb{F}$, so that $\left[\dot{f}_{j+1}, x_{\beta}-c e_{\beta}\right]=0$. Since $\mathfrak{g}_{-1}$ is generated as a $\mathfrak{g}_{0}$-module by $\left\{f_{0}, f_{1}, \ldots, f_{m}\right\}$, it follows from transitivity that $\left[\dot{f}_{j}, x_{\beta}-c e_{\beta}\right]=\zeta e_{\beta-\dot{\alpha}_{j}} \neq 0$. Then

$$
\left[\dot{f}_{j+1},\left[\dot{f}_{j+1},\left[\dot{f}_{j}, x_{\beta}-c e_{\beta}\right]\right]\right]=\zeta\left[\dot{f}_{j+1},\left[\dot{f}_{j+1}, e_{\beta-\dot{\alpha}_{i}}\right]\right] \neq 0
$$

since $\beta-\dot{\alpha}_{j}-2 \dot{\alpha}_{j+1}$ is a root of $\widetilde{\mathfrak{g}}_{0}$. However,

$$
\left[\dot{f}_{j+1},\left[\dot{f}_{j+1},\left[\dot{f}_{j}, x_{\beta}-c e_{\beta}\right]\right]\right]=\left[\left[\dot{f}_{j+1},\left[\dot{f}_{j+1}, \dot{f}_{j}\right]\right], x_{\beta}-c e_{\beta}\right]=0 .
$$

This contradiction shows that $\Phi(\widetilde{\mathfrak{g}})$ is neither $\mathrm{B}_{m+1}$ nor $\mathrm{C}_{m+1}$.

Suppose finally that $\Phi(\mathfrak{g}) \cong \mathrm{F}_{4}$. We will adopt the labeling of roots compatible with [Bou1, Planche VIII]. Because the coefficient $b_{0}$ of $\alpha_{0}$ in $\beta$ is $\geq 2$, we need only consider roots having some coefficient larger than 1 . Below we list the quadruple of coefficients of such roots first relative to the simple roots $\dot{\alpha}_{j}$, then relative to the fundamental weights $\varpi_{j}$. Translation between the two can be accomplished using the fact that $\dot{\alpha}_{i}=\sum_{j} A_{i, j} \varpi_{j}$.

$$
\begin{array}{cccccccc}
\text { root } & 0,1,2,0 & 1,1,2,0 & 0,1,2,1 & 1,2,2,0 & 1,1,2,1 & 0,1,2,2 & 1,2,2,1 \\
\text { weight } & -1,0,2,-2 & 1,-1,2,-2 & -1,0,1,0 & 0,1,0,-2 & 1,-1,1,0 & -1,0,0,2 & 0,1,-1,0 \\
& & & & & & & \\
\text { root } & 1,1,2,2 & 1,2,3,1 & 1,2,2,2 & 1,2,3,2 & 1,2,4,2 & 1,3,4,2 & 2,3,4,2 \\
\text { weight } & 1,-1,0,2 & 0,0,1,-1 & 0,1,-2,2 & 0,0,0,1 & 0,-1,2,0 & -1,1,0,0 & 1,0,0,0
\end{array}
$$

Now $\beta-\dot{\alpha}_{j}$ is a root if and only if either the $j$ th coordinate in the weight expression of $\beta$ is positive or $\beta+\alpha_{j}$ is a root and the $j$ th coordinate in the weight expression of $\beta$ is zero. Thus, the only roots having $|\widetilde{\Delta}(\beta)|>1$ are

$$
\begin{array}{cccccc}
\text { root } & 1,2,3,2 & 1,2,2,2 & 1,1,2,2 & 1,2,2,1 & 1,1,2,1 \\
\widetilde{\Delta}(\beta) & \left\{\dot{\alpha}_{3}, \dot{\alpha}_{4}\right\} & \left\{\dot{\alpha}_{2}, \dot{\alpha}_{4}\right\} & \left\{\dot{\alpha}_{1}, \dot{\alpha}_{4}\right\} & \left\{\dot{\alpha}_{2}, \dot{\alpha}_{4}\right\} & \left\{\dot{\alpha}_{1}, \dot{\alpha}_{3}, \dot{\alpha}_{4}\right\} \\
& & & & & \\
\text { root } & 0,1,2,1 & 1,1,2,0 & & & \\
\widetilde{\Delta}(\beta) & \left\{\dot{\alpha}_{3}, \dot{\alpha}_{4}\right\} & \left\{\dot{\alpha}_{1}, \dot{\alpha}_{3}\right\} . & & &
\end{array}
$$


Since there is a coefficient equal to 0 in each of the last two roots, we may view the calculations for them as occurring in a root system of type $\mathrm{C}_{3}$ or $\mathrm{B}_{3}$. So by the same reasoning as in those cases, those possibilities for $\beta$ can be eliminated. For the roots corresponding to the tuples $(1,2,2,2)$, $(1,1,2,2),(1,2,2,1),(1,1,2,1)$, there are two orthogonal roots in $\widetilde{\Delta}(\beta)$. Thus, an argument as in (3.23) using the $f_{k}, f_{l}$ corresponding to those two roots can be employed in those cases.

Finally, if the root $\beta$ has coefficient tuple $(1,2,3,2), \widetilde{\Delta}(\beta)=\left\{\dot{\alpha}_{3}, \dot{\alpha}_{4}\right\}$, and both $\beta-\dot{\alpha}_{3}-\dot{\alpha}_{4}$ and $\beta-\dot{\alpha}_{3}-2 \dot{\alpha}_{4}$ are roots of $\tilde{\mathfrak{g}}$. However, $\dot{\alpha}_{3}+2 \dot{\alpha}_{4}$ is not a root, so that $\left[\dot{f}_{4},\left[\dot{f}_{4}, \dot{f}_{3}\right]\right]=0$. Then a calculation such as that in (3.28) can be done to rule out this case. Thus, $\Phi(\widetilde{\mathfrak{g}})$ cannot be $\mathrm{F}_{4}$ either.

We have proven that if $\left[f_{l}, x_{\beta}\right] \neq 0$, then either $\beta \notin \Phi(\widetilde{\mathfrak{g}})$ or $\left[f_{l}, e_{\beta}\right]=0$. Because $\left[f_{l}, x_{\beta}\right]=e_{\gamma}$, where $\gamma=\beta-\alpha_{l}$, we have $\left[\left[f_{l}, x_{\beta}\right], e_{-\gamma}\right]=h_{\gamma}$, and it follows that

$$
h_{\gamma}=\left[\left[f_{l}, e_{-\gamma}\right], x_{\beta}\right]+\left[f_{l},\left[x_{\beta}, e_{-\gamma}\right]\right] .
$$

If $\beta \notin \Phi\left(\widetilde{\mathfrak{g}}_{0}\right)$ (the root system of $\widetilde{\mathfrak{g}}_{0}$ ), then since $-\alpha_{l}-\gamma=-\beta$, it must be that $\left[f_{l}, e_{-\gamma}\right]=0$, and the first summand in the expression for $h_{\gamma}$ vanishes. If instead $\beta \in \Phi\left(\widetilde{\mathfrak{g}}_{0}\right)$, then $\left[f_{l}, e_{\beta}\right]=0$, and we can replace $x_{\beta}$ by a suitable linear combination $x_{\beta}+c e_{\beta}, c \in \mathbb{F}$ to get $\left[\left[f_{l}, e_{-\gamma}\right], x_{\beta}\right]=0$. (Such a replacement does not render the equality $\left[\left[f_{l}, x_{\beta}\right], e_{-\gamma}\right]=h_{\gamma}$ invalid, because we have shown that $\left[f_{l}, e_{\beta}\right]=0$.)

Thus, we may assume that $h_{\gamma}=\left[f_{l},\left[x_{\beta}, e_{-\gamma}\right]\right]$. As $\left[f_{l},\left[x_{\beta}, e_{-\gamma}\right]\right] \in \mathbb{F} h_{\alpha_{l}}$, it must be that $h_{\gamma} \in \mathbb{F} h_{\alpha_{l}}$. But then $\varpi_{i}\left(h_{\gamma}\right)=0$ for all $i \neq l$. Since $p>3$, Bourbaki's tables show that either $\gamma=\alpha_{l}$ or $\gamma=-\alpha_{l}$; see [Bou1]. Since $\gamma \in \Phi(\widetilde{\mathfrak{g}})^{+}$, we conclude that $\gamma=\alpha_{l}$. As $\operatorname{deg}\left(e_{\gamma}\right) \geq t-1 \geq 1$, it follows that $l=0$; consequently, $\beta=n \alpha_{0}$ for some integer $n$. However, since $\left[f_{0}, x_{\beta}\right]$ is a root vector of a classical Lie algebra, $n$ must be two; i.e.,

$$
\beta=2 \alpha_{0} \quad(\text { and } t=2) \text {. }
$$

This implies that $x_{\beta}$ is a $\mathfrak{b}^{-}$-primitive vector of $\mathfrak{g}_{t}$ (because $2 \alpha_{0}-\alpha_{j} \notin$ $\left.\Phi\left(\widetilde{\mathfrak{g}}_{0}\right), 1 \leq j \leq m\right)$. Now take any $\nu \in\{1, \ldots, m\}$ for which $\left(\alpha_{\nu}, \alpha_{0}\right) \neq 0$. Then, since $\left[f_{0}, x_{\beta}\right]=e_{0}$ and $\left[f_{\nu}, x_{\beta}\right]=0$, we have 


$$
\begin{aligned}
{\left[\left[f_{0},\left[f_{0}, f_{\nu}\right]\right], x_{\beta}\right] } & =\left(\operatorname{ad}\left[f_{0},\left[f_{0}, f_{\nu}\right]\right]\right)\left(x_{\beta}\right) \\
& =\left[F_{0},\left[F_{0}, F_{\nu}\right]\right]\left(x_{\beta}\right) \quad\left(\text { where } F_{0}=\operatorname{ad} f_{0}, F_{\nu}=\operatorname{ad} f_{\nu}\right) \\
& =\sum_{i=0}^{2}(-1)^{i}\left(\begin{array}{c}
2 \\
i
\end{array}\right) F_{0}^{2-i} F_{\nu} F_{0}^{i}\left(x_{\beta}\right) \\
& =F_{\nu} F_{0}^{2}\left(x_{\beta}\right)=F_{\nu} F_{0}\left(e_{0}\right) \\
& =\left[h_{0}, f_{\nu}\right]=-\alpha_{\nu}\left(h_{\alpha_{0}}\right) f_{\nu} \\
& =-\frac{2\left(\alpha_{\nu}, \alpha_{0}\right)}{\left(\alpha_{0}, \alpha_{0}\right)} f_{\nu} \neq 0
\end{aligned}
$$

whence $2 \alpha_{0}+\alpha_{\nu} \in \Phi\left(\widetilde{\mathfrak{g}}_{0}\right)$. Since $\alpha_{0}$ and $\alpha_{\nu}$ are simple roots, the classical Lie algebra $\widetilde{\mathfrak{g}}$ must have a root system of type $\mathrm{B}_{m+1}, \mathrm{C}_{m+1}, m \geq 2, \mathrm{~F}_{4}$, or $\mathrm{G}_{2}$. Thus, we must consider, respectively,
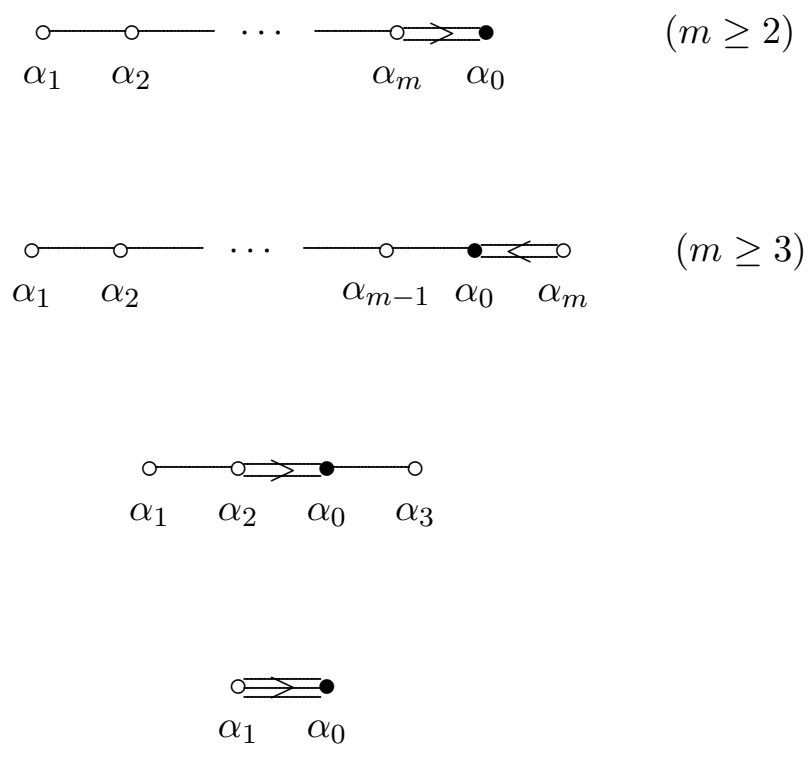

Since we showed that $\left(\alpha_{\nu}, \alpha_{0}\right) \neq 0$ implies that $2 \alpha_{0}+\alpha_{\nu} \in \Phi\left(\widetilde{\mathfrak{g}}_{0}\right)$, neither (3.30) nor (3.31) can occur, since in (3.30), we can take $\nu=m-1$, and in (3.31), we can take $\nu=3$.

Let us now consider (3.29), where the root system is of type $\mathrm{B}_{m+1}$. Recall that in the Cartan matrix for $\mathrm{B}_{m+1}$ (with roots numbered $\dot{\alpha}_{0}, \dot{\alpha}_{1}, \ldots, \dot{\alpha}_{m}$ ), we have $A_{m-1, m}=-2$, so that $\alpha_{m}\left(h_{\alpha_{0}}\right)=\dot{\alpha}_{m-1}\left(h_{\dot{\alpha}_{m}}\right)=A_{m-1, m}=-2$. Then since $\beta=2 \alpha_{0}$ and neither $2 \alpha_{0}-\alpha_{m}$ nor $\alpha_{0}-\alpha_{m}$ is a root, we have 


$$
\begin{aligned}
{\left[\left[f_{0},\left[f_{0},\left[f_{0}, f_{m}\right]\right], x_{\beta}\right]\right.} & =\sum_{i=0}^{3}(-1)^{i}\left(\begin{array}{l}
3 \\
i
\end{array}\right) F_{0}^{3-i} F_{m} F_{0}^{i}\left(x_{\beta}\right) \\
& =\left(3 F_{0} F_{m} F_{0}^{2}-F_{m} F_{0}^{3}\right)\left(x_{\beta}\right) \\
& =\left(3 F_{0} F_{m} F_{0}-F_{m} F_{0}^{2}\right)\left(e_{\alpha_{0}}\right) \\
& =\left(F_{m} F_{0}-3 F_{0} F_{m}\right)\left(h_{\alpha_{0}}\right) \\
& =(2-3(-2))\left[f_{m}, f_{0}\right] \\
& =8\left[f_{0}, f_{m}\right] .
\end{aligned}
$$

However, because $-A_{m-1, m}=2$, the length of the $\dot{\alpha}_{m}=\alpha_{0}$ root string through $\dot{\alpha}_{m-1}=\alpha_{m}$ is 2 , that so $\left[f_{0}, f_{m}\right] \neq 0$, but $\left[f_{0},\left[f_{0},\left[f_{0}, f_{m}\right]\right]\right]=0$. This contradiction shows that the configuration in (3.29) is impossible.

It remains to consider (3.32), where the root system is of type $\mathrm{G}_{2}$. Then $\alpha_{1}\left(h_{\alpha_{0}}\right)=\dot{\alpha}_{2}\left(h_{\dot{\alpha}_{1}}\right)=A_{2,1}=-3$. We calculate

$$
\begin{aligned}
{\left[\left[f_{0},\left[f_{0},\left[f_{0},\left[f_{0}, f_{1}\right]\right]\right], x_{\beta}\right]\right] } & =\sum_{i=0}^{4}(-1)^{i}\left(\begin{array}{l}
4 \\
i
\end{array}\right) F_{0}^{4-i} F_{1} F_{0}^{i}\left(x_{\beta}\right) \\
& =\left(6 F_{0}^{2} F_{1} F_{0}^{2}-4 F_{0} F_{1} F_{0}^{3}+F_{1} F_{0}^{4}\right)\left(x_{\beta}\right) \\
& \left.=\left(-6 F_{0}^{2} F_{1}+4 F_{0} F_{1} F_{0}-F_{1} F_{0}\right)^{2}\right)\left(h_{\alpha_{0}}\right) \\
& =-6 \alpha_{1}\left(h_{\alpha_{0}}\right)\left[f_{0},\left[f_{0}, f_{1}\right]\right]+8\left[f_{0},\left[f_{1}, f_{0}\right]\right] \\
& =(18-8)\left[f_{0},\left[f_{0}, f_{1}\right]\right]=10\left[f_{0}\left[f_{0}, f_{1}\right]\right] .
\end{aligned}
$$

Since the length of the $\dot{\alpha}_{1}=\alpha_{0}$ root string through $\dot{\alpha}_{2}=\alpha_{1}$ is $-A_{2,1}=3$, $\left[f_{0},\left[f_{0}, f_{1}\right]\right] \neq 0$, but $\left[f_{0},\left[f_{0},\left[f_{0},\left[f_{0}, f_{1}\right]\right]\right]\right]=0$. It follows that $(3.32)$ is possible only if $p=5$.

Let $\mathfrak{g}^{\geq-1}$ denote the subalgebra of $\mathfrak{g}$ generated by the graded components $\mathfrak{g}_{i}, i \geq-1$ and let $\mathcal{G}=\bigoplus_{j=-3}^{3} \mathcal{G}_{j}$ be the graded Lie algebra of type $\mathrm{G}_{2}$ from Section 2.14. The above discussion shows that $\bigoplus_{j \leq 0} \mathfrak{g}_{j}^{\geq-1} \cong \bigoplus_{j \leq 0} \mathcal{G}_{j}$ as graded Lie algebras. Clearly, $\mathfrak{g}^{\geq-1}$ is both transitive and 1-transitive. Recall that $x_{\beta} \notin \widetilde{\mathfrak{g}}_{2}$. Therefore, $\operatorname{dim} \mathfrak{g}_{2}^{\geq-1}>\operatorname{dim} \mathcal{G}_{2}=1$, implying $\mathfrak{g}^{\geq-1} \not \mathfrak{G}$. Now Proposition 2.84 enables us to deduce that $\mathfrak{g}^{\geq-1}$ is isomorphic to a Melikyan algebra $M:=M(2 ; \underline{n})$ with its natural grading (as in Section 2.14).

Suppose $\mathfrak{g} \neq \mathfrak{g}^{\geq-1}$. Let $\mathfrak{g}^{\leq 1}$ denote the subalgebra of $\mathfrak{g}$ generated by the graded components $\mathfrak{g}_{i}, i \leq 1$. Set $\mathfrak{g}_{i}^{\leq 1}:=\mathfrak{g}_{-i} \cap \mathfrak{g}^{\leq 1}$. Then, since $\mathfrak{g}$ is 1-transitive, the graded Lie algebra $\mathfrak{g}^{\leq 1}=\bigoplus_{i \in \mathbb{Z}} \mathfrak{g}_{i}^{\leq 1}$ satisfies conditions (i) through (v). Furthermore, because $\mathfrak{g} \neq \mathfrak{g}^{\geq-1}$, it follows that $\mathfrak{g}^{\leq 1} \neq \widetilde{\mathfrak{g}}_{0}$. Reasoning as above, we now conclude that the graded Lie algebra $\mathfrak{g}^{\leq 1}=$ 
$\bigoplus_{i \in \mathbb{Z}} \mathfrak{g}_{i}^{\leq 1}$ is isomorphic to a Melikyan algebra $M^{\prime}=M\left(2 ; \underline{n}^{\prime}\right)$ with its natural grading.

Set

$$
M_{\overline{0}}:=\bigoplus_{i \equiv 0} \mathfrak{g}_{\bmod 3}^{\geq-1} \text { and } M_{\overline{ \pm 2}}:=\bigoplus_{i \equiv \pm 2} \mathfrak{g}_{i}^{\geq-1}
$$

Then, as in Section $2.14, M_{\overline{0}}$ is isomorphic to the Witt Lie algebra $W(2 ; \underline{n})$, and the subspaces $M_{-2}$ and $M_{\overline{2}}$ are irreducible $M_{\overline{0}}$-modules. More precisely, $M_{\overline{2}} \cong \widetilde{W}(2 ; \underline{n})=\{\widetilde{E} \mid E \in W(2 ; \underline{n})\}$ and $M_{-2} \cong \mathcal{O}(2 ; \underline{n})$, and the multiplication is as in (2.82):

$$
\begin{gathered}
{[D, \widetilde{E}]=\widetilde{[D, E]}+2 \operatorname{div}(D) \widetilde{E}} \\
{[D, f]=D(f)-2 \operatorname{div}(D) f} \\
{\left[f_{1} \widetilde{D_{1}}+f_{2} \widetilde{D_{2}}, g_{1} \widetilde{D_{1}}+g_{2} \widetilde{D_{2}}\right]=f_{1} g_{2}-f_{2} g_{1}} \\
{[f, \widetilde{E}]=f E} \\
{[f, g]=2\left(f \widetilde{D}_{g}-g \widetilde{D}_{f}\right) \quad \text { where }} \\
\widetilde{D}_{f}=D_{1}(f) \widetilde{D_{2}}-D_{2}(f) \widetilde{D_{1}} .
\end{gathered}
$$

As in Section 2.14, the algebra $M$ may be assigned a $\mathbb{Z}$-gradation according to:

$$
\begin{gathered}
M_{-3}=\mathbb{F} D_{1} \oplus \mathbb{F} D_{2}, \quad M_{-2}=\mathbb{F} 1, \quad M_{-1}=\mathbb{F} \widetilde{D_{1}} \oplus \widetilde{F} \widetilde{D_{2}} \\
M_{0}=\operatorname{span}_{\mathbb{F}}\left\{x_{i} D_{j} \mid i, j=1,2\right\} \\
M_{1}=\mathbb{F} x_{1}^{(1)} \oplus \mathbb{F} x_{2}^{(1)},
\end{gathered}
$$

Using these results, it is easy to check that the following hold:

$$
\begin{aligned}
& {\left[x_{1}^{(1)} D_{1}-x_{2}^{(1)} D_{2}, x_{1}^{(1)} D_{2}\right]=2 x_{1}^{(1)} D_{2},} \\
& {\left[3 x_{2}^{(1)} D_{2}, x_{2}^{(1)}\right]=3 x_{2}^{(1)}-2 \cdot 3 \cdot x_{2}^{(1)} \equiv 2 x_{2}^{(1)} \bmod 5,} \\
& {\left[x_{1}^{(1)} D_{1}-x_{2}^{(1)} D_{2}, x_{2}^{(1)} D_{1}\right]=-2 x_{1}^{(1)} D_{2},} \\
& {\left[3 x_{2}^{(1)} D_{2}, 3 \widetilde{D_{2}}\right]=-9 \widetilde{D_{2}}+2 \cdot 3 \cdot 3 \widetilde{D_{2}} \equiv-\widetilde{D_{2}} \bmod 5,} \\
& {\left[x_{1}^{(1)} D_{2}, x_{2}^{(1)} D_{1}\right]=x_{1}^{(1)} D_{1}-x_{2}^{(1)} D_{2},} \\
& {\left[x_{2}^{(1)}, 3 \widetilde{D_{2}}\right]=3 x_{2}^{(1)} D_{2}, \text { and }} \\
& {\left[x_{1}^{(1)} D_{2}, \widetilde{D_{2}}\right]=\left[x_{2}^{(1)} D_{1}, x_{2}^{(1)}\right]=0 .}
\end{aligned}
$$


Thus, we can put

$$
\begin{aligned}
e_{1} & :=x_{1}^{(1)} D_{2}, \\
f_{1} & :=x_{2}^{(1)} D_{1}, \\
h_{1} & :=x_{1}^{(1)} D_{1}-x_{2}^{(1)} D_{2} \\
e_{0} & :=x_{2}^{(1)} \\
f_{0} & :=3 \widetilde{D_{2}} \\
h_{0} & :=3 x_{2}^{(1)} D_{2} .
\end{aligned}
$$

Set $x_{\beta}:=x_{2}^{(1)} \widetilde{D_{1}}$. Then

$$
\begin{aligned}
{\left[f_{1}, x_{\beta}\right] } & =0 \\
{\left[h_{1}, x_{\beta}\right] } & =-2 x_{\beta}, \\
{\left[f_{0}, x_{\beta}\right] } & =\left[3 \widetilde{D_{2}}, x_{2}^{(1)} \widetilde{D_{1}}\right]=-3 x_{2}^{(1)} \equiv 2 e_{0} \bmod 5 \\
{\left[h_{0}, x_{\beta}\right] } & =9 x_{2}^{(1)} \widetilde{D_{1}} \equiv 4 x_{\beta} \quad \bmod 5,
\end{aligned}
$$

Since $\widetilde{\mathfrak{g}}_{0} \neq \mathfrak{g}^{\leq 1}$, there exists by symmetry an $x_{-\beta} \in \mathfrak{g}_{-2}$ satisfying the following conditions

$$
\begin{aligned}
{\left[e_{1}, x_{-\beta}\right] } & =0, \\
{\left[h_{1}, x_{-\beta}\right] } & =2 x_{-\beta}, \\
{\left[e_{0}, x_{-\beta}\right] } & =-2 f_{0}, \\
{\left[h_{0}, x_{-\beta}\right] } & =-4 x_{-\beta} .
\end{aligned}
$$

(Recall that $\mathfrak{g}_{-2}=\mathfrak{g}_{2}^{\leq 1}$ and that the role of the $f_{i}$ 's is played in this case by the $e_{i}$ 's, $i=1,2$.)

We claim that $\left[x_{\beta}, x_{-\beta}\right]=0$. Indeed, let $\mathfrak{t}^{\prime}:=\mathbb{F} x_{1}^{(1)} D_{1} \oplus \mathbb{F} x_{2}^{(1)} D_{2}$. Then $h:=\left[x_{\beta}, x_{-\beta}\right]$ is in $\mathfrak{t}^{\prime},\left[x_{1}^{(1)} \widetilde{D_{1}}, x_{\beta}\right]=0,\left[x_{1}^{(1)} \widetilde{D_{1}}, x_{-\beta}\right] \in \mathfrak{g}_{0}$, and

$$
\left[x_{1}^{(1)} D_{1}-x_{2}^{(1)} D_{2},\left[x_{1}^{(1)} \widetilde{D_{1}}, x_{-\beta}\right]\right]=2\left[x_{1}^{(1)} \widetilde{D_{1}}, x_{-\beta}\right]
$$

(see (3.35) above). By comparing eigenvalues relative to $x_{1}^{(1)} D_{1}-x_{2}^{(1)} D_{2}$, we see that $\left[x_{1}^{(1)} \widetilde{D_{1}}, x_{-\beta}\right]=a x_{1}^{(1)} D_{2}$, where $a \in \mathbb{F}$. Therefore,

$$
\begin{aligned}
{\left[x_{1}^{(1)} \widetilde{D_{1}},\left[x_{\beta}, x_{-\beta}\right]\right] } & =\left[x_{\beta},\left[x_{1}^{(1)} \widetilde{D_{1}}, x_{-\beta}\right]\right] \\
& =\left[x_{2}^{(1)} \widetilde{D_{1}}, a x_{1}^{(1)} D_{2}\right] \\
& =a\left(x_{2}^{(1)} \widetilde{D_{2}}-x_{1}^{(1)} \widetilde{D_{1}}\right) .
\end{aligned}
$$

On the other hand, $\left[x_{1}^{(1)} \widetilde{D_{1}},\left[x_{\beta}, x_{-\beta}\right]\right]=-\left[h, x_{1}^{(1)} \widetilde{D_{1}}\right] \in \mathbb{F} x_{1}^{(1)} \widetilde{D_{1}}$. This implies that $a=0$, so that $\left[x_{1}^{(1)} \widetilde{D_{1}}, x_{-\beta}\right]=0=\left[h, x_{1}^{(1)} \widetilde{D_{1}}\right]$. Since $h \in \mathfrak{t}^{\prime}$ 
and $\left[x_{1}^{(1)} D_{1}, x_{1}^{(1)} \widetilde{D_{1}}\right]=\left[x_{2}^{(1)} D_{2}, x_{1}^{(1)} \widetilde{D_{1}}\right]=2 x_{1}^{(1)} \widetilde{D_{1}}$, we obtain that $h=$ $b\left(x_{1}^{(1)} D_{1}-x_{2}^{(1)} D_{2}\right)$ for some $b \in \mathbb{F}$.

Suppose that $b \neq 0$, and set $e_{1}=x_{1}^{(1)} D_{2}, f_{1}=x_{2}^{(1)} D_{1}$ as above, $e_{2}:=$ $b^{-1} x_{-\beta}$, and $f_{2}:=x_{\beta}$. Then $\left[e_{i}, f_{j}\right]=\delta_{i, j} h_{\alpha_{1}}, 1 \leq i, j \leq 2$, and $\left[h_{\alpha_{1}}, e_{i}\right]=$ $2 e_{i}, i=1,2$, and $\left[h_{\alpha_{1}}, f_{i}\right]=-2 f_{i}, i=1,2$. Then by Theorem 3.7, the Lie algebra generated by the elements $e_{i}, f_{i} \quad i=1,2$, would be infinitedimensional, so that we must have that $b=0$ and $h=\left[x_{\beta}, x_{-\beta}\right]=0$.

Set $e_{1}^{\prime}:=\left[x_{\beta}, e_{0}\right]$ and $f_{1}^{\prime}:=\left[x_{-\beta}, f_{0}\right]$. By $(3.34)$ we have $\left[\left[x_{\beta}, e_{0}\right], f_{0}\right] \in$ $\mathbb{F} x_{\beta}$. This yields

$$
\begin{aligned}
{\left[e_{1}^{\prime}, f_{1}^{\prime}\right] } & =\left[\left[x_{\beta}, e_{0}\right],\left[x_{-\beta}, f_{0}\right]\right]=\left[\left[\left[x_{\beta}, e_{0}\right], x_{-\beta}\right], f_{0}\right] \\
& =\left[\left[x_{\beta},\left[e_{0}, x_{-\beta}\right]\right], f_{0}\right]=-2\left[\left[x_{\beta}, f_{0}\right], f_{0}\right] \\
& =4\left[e_{0}, f_{0}\right]=4 h_{\alpha_{0}} .
\end{aligned}
$$

Using the results of Section 2.14, one sees that the $\mathfrak{g}_{0}^{(1)}$-modules $\mathfrak{g}_{4}^{\geq-1}$ and $\mathfrak{g}_{4}^{\leq 1}$ map into the graded component $\mathcal{O}(2)_{2}$, and so, as in the proof of Lemma 2.78 (b), are isomorphic to the 3 -dimensional $\mathfrak{g}_{0}^{(1)}$-module $L(2)$. Inasmuch as $\left[e_{1}^{\prime}, e_{0}\right] \in \mathfrak{g}_{4}^{\geq-1}$ and $\left[f_{1}^{\prime}, f_{0}\right] \in \mathfrak{g}_{4}^{\leq 1}$, and $\left[h_{\alpha_{1}}, e_{0}\right]=-e_{0}$ (see (3.34)), we have

$$
\left.\left.\left[h_{\alpha_{1}},\left[e_{1}^{\prime}, e_{0}\right]\right]=\left[h_{\alpha_{1}},\left[\left[x_{\beta}, e_{0}\right], e_{0}\right]\right]=-4\left[e_{1}^{\prime}, e_{0}\right]\right] \equiv\left[e_{1}^{\prime}, e_{0}\right]\right] \bmod 5,
$$

and, by symmetry,

$$
\left[h_{\alpha_{1}},\left[f_{1}^{\prime}, f_{0}\right]\right]=-\left[f_{1}^{\prime}, f_{0}\right] \bmod 5 .
$$

Comparing eigenvalues, we see that $\left[e_{1}^{\prime}, e_{0}\right]=\left[f_{1}^{\prime}, f_{0}\right]=0$.

Now set $e_{2}^{\prime}:=e_{0}, f_{2}^{\prime}:=f_{0}, h^{\prime}:=h_{\alpha_{0}}, e_{3}^{\prime}:=f_{1}^{\prime}$, and $f_{3}^{\prime}:=e_{1}^{\prime}$. Then $\left[e_{i}^{\prime}, f_{j}^{\prime}\right]=\delta_{i, j} h^{\prime}, j=2,3$, and $\left[h^{\prime}, e_{2}^{\prime}\right]=2 e_{2}^{\prime},\left[h^{\prime}, f_{2}^{\prime}\right]=-2 f_{2}^{\prime},\left[h^{\prime}, e_{3}^{\prime}\right]=\left[h^{\prime}, f_{1}^{\prime}\right]$ $=\left[h_{\alpha_{0}},\left[x_{-\beta}, f_{0}\right]\right]=-4 e_{3}^{\prime}=e_{3}^{\prime} \bmod 5$, and, by symmetry, $\left[h^{\prime}, f_{3}^{\prime}\right]=-f_{3}^{\prime}$. Therefore, Theorem 3.7 applies, showing that the Lie algebra generated by $e_{i}^{\prime}, f_{i}^{\prime}, i=2,3$, is infinite-dimensional.

Thus, we have proved that if $\mathfrak{g}_{t} \neq \widetilde{\mathfrak{g}}_{t}$ for some $t \geq 2$, then $p=5$, and $\mathfrak{g}$ is isomorphic to a Melikyan algebra with the natural grading. If $\mathfrak{g}_{t}=\widetilde{\mathfrak{g}}_{t}$ for all $t \geq 2$ and $\mathfrak{g}_{-s} \neq \widetilde{\mathfrak{g}}_{-s}$ for some $s>0$, we deal with the opposite grading

$$
\mathfrak{g}=\bigoplus_{i=-r}^{q} \mathfrak{g}_{i}^{\prime},
$$

where $\mathfrak{g}_{i}^{\prime}=\mathfrak{g}_{-i}$. Since $\mathfrak{g}_{1}$ is an irreducible restricted $\mathfrak{g}_{0}^{(1)}$-module, this graded Lie algebra satisfies the conditions of Theorem 3.22. By the same reasoning as above, one can prove now that again $p=5, \mathfrak{g}$ is a Melikyan algebra, and the grading of $\mathfrak{g}$ is the opposite of the natural grading.

This completes the proof of Theorem 3.22 and with it the contragredient case of the Main Theorem. 


\section{CHAPTER 4}

\section{The Noncontragredient Case}

\subsection{General assumptions and notation}

Throughout this chapter, in which we prove the noncontragredient case of the Main Theorem, our blanket assumptions are

(1) $\mathfrak{g}=\bigoplus_{j=-q}^{r} \mathfrak{g}_{j}$ is a finite-dimensional graded Lie algebra over an algebraically closed field $\mathbb{F}$ of characteristic $p>3$ with $\mathfrak{g}_{1} \neq 0$;

(2) $\mathfrak{g}_{0}$ is a classical reductive Lie algebra, and $\mathfrak{g}_{0}=\mathfrak{g}_{0}^{[1]} \oplus \cdots \oplus \mathfrak{g}_{0}^{[\ell]}$ is a decomposition of $\mathfrak{g}_{0}$ into ideals $\mathfrak{g}_{0}^{[i]}$ which are

(i) classical simple,

(ii) $\mathfrak{s l}_{n}, \mathfrak{g l}_{n}$, or $\mathfrak{p g l}_{n}$ where $p \mid n$, or

(iii) one-dimensional.

Also throughout our conventions are

(a) $\mathfrak{t}$ is a fixed maximal toral subalgebra in $\mathfrak{g}_{0}$ and $\mathfrak{t}^{[i]}=\mathfrak{t} \cap \mathfrak{g}_{0}^{[i]}$;

(b) $\Phi$ is the root system of $\mathfrak{g}_{0}$ with respect to $\mathfrak{t} ; \quad \Delta=\left\{\alpha_{1}, \ldots, \alpha_{m}\right\}$ is a system of simple roots in $\Phi$; and $\Phi^{+}$and $\Phi^{-}$are the positive and negative roots respectively relative to $\Delta$;

(c) for $\alpha \in \Phi$, the root vector $e_{\alpha}$ spans the root space $\mathfrak{g}_{0}^{\alpha}$, and the triple of vectors $\left(e_{\alpha}, e_{-\alpha}, h_{\alpha}\right)$, where $h_{\alpha} \in \mathfrak{t}$, form a standard basis for a copy of $\mathfrak{s l}_{2}$ :

$$
\left[e_{\alpha}, e_{-\alpha}\right]=h_{\alpha}, \quad\left[h_{\alpha}, e_{\alpha}\right]=2 e_{\alpha}, \quad\left[h_{\alpha}, e_{-\alpha}\right]=-2 e_{-\alpha} .
$$

(If $\left(e_{\alpha}, e_{-\alpha}, h_{\alpha}\right)$ is the triple corresponding to $\alpha \in \Phi^{+}$, then we assume that the triple $\left(e_{-\alpha}, e_{\alpha}, h_{-\alpha}=-h_{\alpha}\right)$ is the one corresponding to $-\alpha \in \Phi^{-}$. One of the vectors $e_{\alpha}$ or $e_{-\alpha}$ may be chosen to be an arbitrary nonzero root vector, and then the remaining vectors in the triple are chosen accordingly.)

(d) $\mathfrak{n}^{+}=\bigoplus_{\alpha \in \Phi^{+}} \mathfrak{g}_{0}^{\alpha}, \mathfrak{n}^{-}=\bigoplus_{\alpha \in \Phi^{-}} \mathfrak{g}_{0}^{\alpha}, \mathfrak{b}^{+}=\mathfrak{t} \oplus \mathfrak{n}^{+}$, and $\mathfrak{b}^{-}=\mathfrak{t} \oplus \mathfrak{n}^{-}$.

(e) (, ) is a symmetric bilinear form on the dual space to $\mathfrak{t} \cap \mathfrak{g}_{0}^{(1)}$ which is invariant under the Weyl group $W$ of $\mathfrak{g}_{0}^{(1)}$ and whose restriction to each subspace $\left(\mathfrak{t}^{[i]} \cap \mathfrak{g}_{0}^{(1)}\right)^{*}$ of $\left(\mathfrak{t} \cap \mathfrak{g}_{0}^{(1)}\right)^{*}$ is nonzero. (Such a form can be obtained by mod $p$ reduction of the $W$-invariant form used in the tables at the end of [Bou1].) 
Weight vectors in $\mathfrak{g}$ will be relative to the toral subalgebra $\mathfrak{t}$. Let $\mathfrak{g}_{i}^{\lambda}=\left\{x \in \mathfrak{g}_{i} \mid[t, x]=\lambda(t) x\right.$ for all $\left.t \in \mathfrak{t}\right\}$ denote the weight space in $\mathfrak{g}_{i}$ corresponding to $\lambda \in \mathfrak{t}^{*}$. Let $\varpi_{1}, \ldots, \varpi_{m}$ be the fundamental weights relative to our system of simple roots $\Delta$ so that $\varpi_{i}\left(h_{\alpha_{j}}\right)=\left\langle\varpi_{i}, \alpha_{j}\right\rangle=\delta_{i, j}$. Then any weight of $\mathfrak{t}$ is a linear combination of the $\varpi_{i}$ 's.

The classical Lie algebras of types $\mathrm{A}_{m}$ and $\mathrm{C}_{m}$ play a special role in what follows. Often we identify an algebra of type $\mathrm{A}_{m}$ with $\mathfrak{s l}(V)$ (or $\mathfrak{s l}(V)$ modulo its center) and an algebra of type $\mathrm{C}_{m}$ with $\mathfrak{s p}(V) \subseteq \mathfrak{s l}(V)$. In the A-case, the natural module $V$ has dimension $m+1$ and weights $\varepsilon_{i}(1 \leq i \leq m+1)$ relative to the toral subalgebra of diagonal matrices, where $\varepsilon_{i}$ denotes the projection of a matrix onto its $(i, i)$-entry. Thus, $\varepsilon_{1}+\cdots+\varepsilon_{m+1}=0$ on $\mathfrak{s l}(V)$, and the weights of $V$ are $\varpi_{1}, \varpi_{2}-\varpi_{1}, \ldots, \varpi_{m}-\varpi_{m-1},-\varpi_{m}$ in the language of the fundamental weights. In the C-case, $V$ has dimension $2 m$ and weights $\pm \varepsilon_{i}(1 \leq i \leq m)$, or in terms of the fundamental weights, $\pm \varpi_{1}, \pm\left(\varpi_{2}-\varpi_{1}\right), \ldots, \pm\left(\varpi_{m}-\varpi_{m-1}\right)$. We will adopt the notation in [Bou1] giving the expressions for the roots in terms of the weights $\varepsilon_{i}$ whenever we consider the root systems of type $\mathrm{A}_{m}$ and $\mathrm{C}_{m}$. Thus for type $\mathrm{A}_{m}$, we have

$$
\begin{aligned}
& \Phi=\left\{\varepsilon_{i}-\varepsilon_{j} \mid 1 \leq i, j \leq m+1, i \neq j\right\}, \\
& \Delta=\left\{\alpha_{i}=\varepsilon_{i}-\varepsilon_{i+1} \mid 1 \leq i \leq m\right\},
\end{aligned}
$$

and for type $\mathrm{C}_{m}, m \geq 2$,

$$
\begin{gathered}
\Phi=\left\{ \pm \varepsilon_{i} \pm \varepsilon_{j} \mid 1 \leq i, j \leq m\right\}, \\
\Delta=\left\{\alpha_{i}=\varepsilon_{i}-\varepsilon_{i+1} \mid 1 \leq i \leq m-1\right\} \cup\left\{\alpha_{m}=2 \varepsilon_{m}\right\} .
\end{gathered}
$$

(Compare the discussion in Section 2.1.) The module $V$ has a unique highest weight, namely $\varpi_{1}$, (relative to the usual partial order in which $\nu \leq \mu$ if $\mu-\nu=\sum_{i=1}^{m} k_{i} \alpha_{i}$, where $k_{i} \in \mathbb{N}$ for all $i$ ), and a unique lowest weight, which is $-\varpi_{m}$ in the $\mathrm{A}_{m}$-case and is $-\varpi_{1}$ in the $\mathrm{C}_{m}$-case. We use the term standard representation to refer to either $V$ or its dual module $V^{*}$. In the $\mathrm{C}_{m}$-case, $V$ is isomorphic to $V^{*}$, but this is not true in the $\mathrm{A}_{m}$-case, since $V^{*}$ has highest weight $\varpi_{m}$ and lowest weight $-\varpi_{1}$.

\subsection{Brackets of weight vectors in opposite gradation spaces}

Here we obtain information about the weights of certain pairs of weight vectors lying in homogeneous spaces of opposite degree. This information

will later help us to analyze the root structure of $\mathfrak{g}_{0}$ and the structure of the $\mathfrak{g}_{0}$-module $\mathfrak{g}_{-1}$.

Lemma 4.3. Suppose for $i \neq 0$ that there are weight vectors $x_{\lambda} \in \mathfrak{g}_{-i}^{\lambda}$ and $x_{\gamma} \in \mathfrak{g}_{i}^{\gamma}$ corresponding to weights $\lambda, \gamma \in \mathfrak{t}^{*}$, which satisfy the following conditions: 


$$
\left[x_{\lambda}, x_{\gamma}\right]=e_{-\delta}, \quad\left[x_{\lambda}, e_{\delta}\right]=0=\left[x_{\gamma}, e_{-\delta}\right] \text { for some } \delta \in \Phi .
$$

Then either $\lambda\left(h_{\delta}\right)=1$ or $\lambda\left(h_{\delta}\right)=0$.

Proof. Assume that $\lambda\left(h_{\delta}\right)\left(\lambda\left(h_{\delta}\right)-1\right) \neq 0$ and set

$$
\begin{aligned}
e_{1} & =\left[x_{\gamma}, e_{\delta}\right], \quad e_{2}=\left[\left[\left[x_{\gamma}, e_{\delta}\right], e_{\delta}\right], e_{\delta}\right], \quad f_{1}=x_{\lambda} \\
f_{2} & \left.=\left(\lambda\left(h_{\delta}\right)\left(\lambda\left(h_{\delta}\right)-1\right)\right)^{-1}\left[\left[x_{\lambda}, e_{-\delta}\right], e_{-\delta}\right]\right]
\end{aligned}
$$

If $\xi:=\left(\lambda\left(h_{\delta}\right)\left(\lambda\left(h_{\delta}\right)-1\right)\right)^{-1}$, then calculation shows that

$$
\begin{aligned}
{\left[e_{1}, f_{1}\right]=} & {\left[\left[x_{\gamma}, x_{\lambda}\right], e_{\delta}\right]=h_{\delta} } \\
{\left[e_{1}, f_{2}\right] \in } & \mathbb{F}\left[\left[x_{\gamma},\left[\left[x_{\lambda}, e_{-\delta}\right], e_{-\delta}\right]\right], e_{\delta}\right]+\mathbb{F}\left[x_{\gamma},\left[e_{\delta},\left[\left[x_{\lambda}, e_{-\delta}\right], e_{-\delta}\right]\right]\right] \\
& \subseteq \mathbb{F}\left[x_{\gamma},\left[x_{\lambda}, e_{-\delta}\right]\right]=0 \\
{\left[e_{2}, f_{1}\right]=} & {\left[\left[\left[\left[x_{\gamma}, x_{\lambda}\right], e_{\delta}\right], e_{\delta}\right], e_{\delta}\right]=0 } \\
{\left[e_{2}, f_{2}\right]=} & \xi\left(X_{\gamma} E_{\delta}^{3}-3 E_{\delta} X_{\gamma} E_{\delta}^{2}+3 E_{\delta}^{2} X_{\gamma} E_{\delta}-E_{\delta}^{3} X_{\gamma}\right)\left(\left[\left[x_{\lambda}, e_{-\delta}\right], e_{-\delta}\right]\right) \\
= & -3 \xi E_{\delta} X_{\gamma} E_{\delta}^{2}\left(\left[\left[x_{\lambda}, e_{-\delta}\right], e_{-\delta}\right]\right) \\
= & -6 E_{\delta} X_{\gamma}\left(x_{\lambda}\right)=6 h_{\delta},
\end{aligned}
$$

where $E_{\delta}=\operatorname{ad} e_{\delta}$ and $X_{\gamma}=\operatorname{ad} x_{\gamma}$. Furthermore, since $e_{1}=\left[x_{\gamma}, e_{\delta}\right]$ and $\lambda=-(\gamma+\delta)$,

$$
\begin{array}{cc}
{\left[h_{\delta}, e_{1}\right]=-\lambda\left(h_{\delta}\right) e_{1},} & {\left[h_{\delta}, e_{2}\right]=\left(-\lambda\left(h_{\delta}\right)+4\right) e_{2}} \\
{\left[h_{\delta}, f_{1}\right]=\lambda\left(h_{\delta}\right) f_{1},} & {\left[h_{\delta}, f_{2}\right]=\left(\lambda\left(h_{\delta}\right)-4\right) f_{2} .}
\end{array}
$$

Since $\lambda\left(h_{\delta}\right) \neq 0$, this contradicts Theorem 3.7, which we apply by replacing $h_{\delta}$ by $-2\left(\lambda\left(h_{\delta}\right)\right)^{-1} h_{\delta}$ and $f_{2}$ by $\frac{1}{6} f_{2}$.

\subsection{Determining $\mathfrak{g}_{0}$ and its representation on $\mathfrak{g}_{-1}$}

Under a few additional assumptions, we are able to prove here that the commutator $\mathfrak{g}_{0}^{(1)}$ must be of type $\mathrm{A}_{m}$ or $\mathrm{C}_{m}$, and that the representation of $\mathfrak{g}_{0}^{(1)}$ on $\mathfrak{g}_{-1}$ is a standard representation.

TheOREM 4.4. Under hypotheses (1)-(2) of Section 4.1, suppose that there exist a $\mathfrak{b}^{+}$-primitive vector $f^{\Lambda} \in \mathfrak{g}_{-1}$ of weight $\Lambda$ and a $\mathfrak{b}^{-}$-primitive vector $e^{\Gamma} \in \mathfrak{g}_{1}$ of weight $\Gamma$ such that

(i) $\left[f^{\Lambda}, e^{\Gamma}\right]=e_{-\alpha}$ for some $\alpha \in \Phi$;

(ii) $\Lambda\left(\mathfrak{t}^{[i]}\right) \neq 0$ for all $i=1, \ldots, \ell$ if $\alpha \in \Phi^{+}$, and $\Gamma\left(\mathfrak{t}^{[i]}\right) \neq 0$ for all $i=1, \ldots, \ell$ if $\alpha \in \Phi^{-}$. 
Then the commutator ideal $\mathfrak{g}_{0}^{(1)}$ of $\mathfrak{g}_{0}$ consists of a single summand, which is of type $\mathrm{A}_{m}$ or $\mathrm{C}_{m}$. Moreover, if $\alpha \in \Phi^{+}$, then $\alpha$ is the highest root of $\Phi$, and $\Lambda$ is the highest weight of a standard representation of $\mathfrak{g}_{0}^{(1)}$. If $\alpha \in \Phi^{-}$, then $\alpha$ is the lowest root of $\Phi$ and $\Gamma$ is the lowest weight of a standard representation of $\mathfrak{g}_{0}^{(1)}$.

REMARK 4.5. The condition $\Lambda\left(\mathfrak{t}^{[i]}\right) \neq 0$ for any $i=1, \ldots, \ell$ holds automatically when $\mathfrak{g}_{-1}$ is an irreducible $\mathfrak{g}_{0}$-module and $\mathfrak{g}$ is transitive. Indeed, if $\Lambda\left(\mathfrak{t}^{[i]}\right)=0$ for some $i$, then $\left[\mathfrak{g}_{0}^{[i]}, f^{\Lambda}\right]=0$, because in this case $\mathfrak{g}_{-1}$ is a completely reducible $\mathfrak{g}_{0}^{[i]}$-module with all composition factors being simple modules generated by $\mathfrak{b}^{+}$-primitive vectors of weight zero. Then $\mathfrak{g}_{-1}=\sum_{j \neq i} \mathfrak{U}\left(\mathfrak{g}_{0}^{[j]}\right) f^{\Lambda}$ by irreducibility. But this implies $\mathfrak{g}_{-1}$ is annihilated by $\mathfrak{g}_{0}^{[i]}$, which contradicts transitivity.

Proof. It suffices to prove the result when $\alpha \in \Phi^{+}$, for the argument in the case $\alpha \in \Phi^{-}$is completely symmetrical and uses the hypothesis $\Gamma\left(\mathfrak{t}^{[i]}\right) \neq 0$ for any $i$.

First we assume that $\alpha$ is a highest root of $\Phi$, hence a highest root of some summand $\mathfrak{g}_{0}^{[k]}$, and we show that then $\mathfrak{g}_{0}^{(1)}$ is of type $\mathrm{A}_{m}$ or $\mathrm{C}_{m}$ and $\Lambda$ is the highest weight of a standard representation of $\mathfrak{g}_{0}^{(1)}$. We begin by proving that $\Lambda\left(h_{\alpha}\right) \neq 0$. Indeed, suppose $\Lambda\left(h_{\alpha}\right)=0$. If $\mathfrak{g}_{0}^{[k]}$ is isomorphic to $\mathfrak{s l}_{2}$, then $\Lambda\left(\mathfrak{t}^{[k]}\right)=0$, contrary to assumption. Therefore, we may assume that the rank of $\mathfrak{g}_{0}^{[k]}$ is $\geq 2$ and may choose $\beta \in \Phi^{+} \backslash\{\alpha\}$ such that $(\alpha, \beta)>0$. Then $\left[e_{\alpha}, e_{-\beta}\right] \neq 0$ in $\mathfrak{g}_{0}$. Consider the weight vectors

$x_{\lambda}=\left[f^{\Lambda}, e_{-\beta}\right], \quad x_{\gamma}=\left[e^{\Gamma}, e_{\beta}\right], \quad e_{-\delta}=(\Gamma+\alpha)\left(h_{\beta}\right) e_{-\alpha}=-\Lambda\left(h_{\beta}\right) e_{-\alpha}$.

Since $f^{\Lambda}$ is a $\mathfrak{b}^{+}$-primitive vector of weight $\Lambda$, and $e^{\Gamma}$ is a $\mathfrak{b}^{-}$-primitive vector of weight $\Gamma$, and $\Lambda+\Gamma=-\alpha$, a lowest root, we have

$$
\begin{aligned}
{\left[x_{\lambda}, x_{\gamma}\right] } & =\left[\left[f^{\Lambda}, e_{-\beta}\right],\left[e^{\Gamma}, e_{\beta}\right]\right] \\
& =\left[\left[\left[f^{\Lambda}, e_{-\beta}\right], e^{\Gamma}\right], e_{\beta}\right]+\left[e^{\Gamma},\left[\left[f^{\Lambda}, e_{-\beta}\right], e_{\beta}\right]\right] \\
& =-\left[e^{\Gamma},\left[f^{\Lambda},\left[e_{\beta}, e_{-\beta}\right]\right]\right. \\
& =\Lambda\left(h_{\beta}\right)\left[e^{\Gamma}, f^{\Lambda}\right]=-\Lambda\left(h_{\beta}\right) e_{-\alpha}=e_{-\delta} .
\end{aligned}
$$

In addition, since $\delta=\alpha$, the highest root of $\mathfrak{g}_{0}^{(1)}$,

$$
\left[x_{\lambda}, e_{\delta}\right]=0=\left[x_{\gamma}, e_{-\delta}\right] .
$$

Therefore, Lemma 4.3 applies and gives $\lambda\left(h_{\delta}\right)=(\Lambda-\beta)\left(h_{\alpha}\right)=0$ or 1 . Since we are assuming that $\Lambda\left(h_{\alpha}\right)=0$, this forces $\beta\left(h_{\alpha}\right)=0$ or $\beta\left(h_{\alpha}\right)=-1$. But $\beta\left(h_{\alpha}\right) \in\{0,1,2,3\}$ as $\alpha$ is a highest root of $\Phi$. Therefore, $\beta\left(h_{\alpha}\right)=0$, which 
implies $(\beta, \alpha)=0$, a contradiction to our choice of $\beta$. We conclude that $\Lambda\left(h_{\alpha}\right) \neq 0$. But then applying Lemma 4.3 to the weight vectors $f^{\Lambda}, e^{\Gamma}$ and $e_{-\alpha}$ gives $\Lambda\left(h_{\alpha}\right)=1$.

Our next goal is to prove that $\Lambda\left(h_{\beta}\right)=0$ for any $\beta \in \Phi^{+}$such that $\alpha-\beta \notin \Phi$. Indeed, if on the contrary, $\Lambda\left(h_{\beta}\right) \neq 0$ for some $\beta \in \Phi^{+}$with $\alpha-\beta \notin \Phi$, we may consider the elements:

$$
\begin{array}{ccc}
e_{1}=\left[e^{\Gamma}, e_{\alpha}\right], & e_{2}=\left[\left[e^{\Gamma}, e_{\alpha}\right], e_{\beta}\right], & \\
f_{1}=f^{\Lambda}, & f_{2}=-\Lambda\left(h_{\beta}\right)^{-1}\left[f^{\Lambda}, e_{-\beta}\right], & h=h_{\alpha} .
\end{array}
$$

Then because $\alpha-\beta \notin \Phi$ (so that $\left[e_{-\beta}, e_{\alpha}\right]=0$ ) and $\alpha$ is a highest root, we have $\alpha\left(h_{\beta}\right)=0$, and hence $\beta\left(h_{\alpha}\right)=0$. Moreover, since $\Gamma+\alpha=-\Lambda$ and $f^{\Lambda}$ (resp. $e^{\Gamma}$ ) is a $\mathfrak{b}^{+}$-primitive vector of weight $\Lambda$ (resp. $\mathfrak{b}^{-}$-primitive vector of weight $\Gamma$ ), we have the following relations:

$$
\begin{aligned}
{\left[e_{1}, f_{1}\right] } & =h_{\alpha}=h, \\
{\left[e_{1}, f_{2}\right] } & =-\Lambda\left(h_{\beta}\right)^{-1}\left[\left[e^{\Gamma}, e_{\alpha}\right],\left[f^{\Lambda}, e_{-\beta}\right]\right] \\
& =-\Lambda\left(h_{\beta}\right)^{-1}\left(\left[\left[\left[e^{\Gamma}, e_{\alpha}\right], f^{\Lambda}\right], e_{-\beta}\right]+\left[f^{\Lambda},\left[\left[e^{\Gamma}, e_{\alpha}\right], e_{-\beta}\right]\right]\right)=0 \\
{\left[e_{2}, f_{1}\right] } & =\left[\left[\left[e^{\Gamma}, f^{\Lambda}\right], e_{\alpha}\right], e_{\beta}\right]=\beta\left(h_{\alpha}\right) e_{\beta}=0, \\
{\left[e_{2}, f_{2}\right] } & =\Lambda\left(h_{\beta}\right)^{-1}\left[\left[f^{\Lambda},\left[e_{-\beta},\left[\left[e^{\Gamma}, e_{\alpha}\right], e_{\beta}\right]\right]\right.\right. \\
& =\Lambda\left(h_{\beta}\right)^{-1}(\Gamma+\alpha)\left(h_{\beta}\right)\left[f^{\Lambda},\left[e^{\Gamma}, e_{\alpha}\right]\right]=h_{\alpha}=h .
\end{aligned}
$$

Furthermore, since $\Lambda\left(h_{\alpha}\right)=1, \Gamma+\alpha=-\Lambda$, and $\beta\left(h_{\alpha}\right)=0$, we have

$$
\left[h, e_{i}\right]=-e_{i} \quad\left[h, f_{i}\right]=f_{i}, \quad i=1,2 .
$$

These calculations show that the elements $h^{\prime}=-2 h, e_{i}^{\prime}=e_{i}, f_{i}^{\prime}=-2 f_{i}$, for $i=1,2$, satisfy all the conditions of Theorem 3.7 and so generate an infinitedimensional algebra. Since $\mathfrak{g}$ is finite-dimensional, this is impossible. Hence $\Lambda\left(h_{\beta}\right)=0$ for any $\beta \in \Phi^{+}$such that $\alpha-\beta \notin \Phi$.

Take a simple root $\alpha_{n}$ in $\Phi^{[i]}$ for some $i \neq k$. Since $\alpha-\alpha_{n} \notin \Phi$, we have $\Lambda\left(h_{\alpha_{n}}\right)=0$ by what we have just shown. But $\Lambda\left(\mathfrak{t}^{[i]}\right) \neq 0$ for all $i=1, \ldots, \ell$. Consequently, $\Phi$ must be an irreducible root system.

If $\beta$ is a simple root for which $\alpha-\beta \in \Phi$ and $\alpha-2 \beta \notin \Phi$, then necessarily $\beta \in \Phi^{[k]}$ and $\alpha-\beta \in\left(\Phi^{[k]}\right)^{+}$. In this case, set

$$
x_{\lambda}=f^{\Lambda}, \quad x_{\gamma}=\left[e^{\Gamma}, e_{\alpha-\beta}\right] .
$$

Then $\left[x_{\lambda}, e_{\beta}\right]=0=\left[x_{\gamma}, e_{-\beta}\right]$. Moreover,

$$
\left[x_{\lambda}, x_{\gamma}\right]=\left[\left[f^{\Lambda}, e^{\Gamma}\right], e_{\alpha-\beta}\right]=\left[e_{-\alpha}, e_{\alpha-\beta}\right]=\zeta e_{-\beta}
$$

for some $\zeta \neq 0$. Thus, $\Lambda\left(h_{\beta}\right)=0$ or $\Lambda\left(h_{\beta}\right)=1$ by Lemma 4.3 for such a $\operatorname{root} \beta$.

Let $\alpha=\sum_{i=1}^{m} k_{i} \alpha_{i}$. Examination of the root systems (see 4.5) shows that when $\Phi \neq \mathrm{A}_{m}$ or $\mathrm{C}_{m}$, there exists $j \in\{1, \ldots, m\}$ such that 
(i) $\alpha-\alpha_{j} \in \Phi$,

(ii) $\alpha-2 \alpha_{j} \notin \Phi$,

(iii) $(\alpha, \alpha)=\left(\alpha_{j}, \alpha_{j}\right)$,

(iv) $k_{j}=2$

(v) $\alpha-\alpha_{i} \notin \Phi$ for all $i \in\{1, \ldots, m\}$ such that $i \neq j$.

Then by $(\mathrm{v})$ and what we have shown, $\Lambda\left(h_{\alpha_{i}}\right)=0$ for $i \neq j$. Since $\mathfrak{g}_{-1}$ is not a trivial $\mathfrak{g}_{0}^{(1)}$-module, we obtain from the preceding paragraph that $\Lambda\left(h_{\alpha_{j}}\right)=1$. But then in view of Theorem 2.3 we have that

$$
\Lambda\left(h_{\alpha}\right)=\Lambda\left(\sum_{i=1}^{n}\left\langle\varpi_{i}, \alpha\right\rangle h_{\alpha_{i}}\right)=\left\langle\varpi_{j}, \alpha\right\rangle \Lambda\left(h_{\alpha_{j}}\right)=k_{j} \Lambda\left(h_{\alpha_{j}}\right)=k_{j}=2,
$$

contradicting the fact that $\Lambda\left(h_{\alpha}\right)=1$. It must be then that $\Phi=\mathrm{A}_{m}$ or $\Phi=\mathrm{C}_{m}$.

If $\Phi=\mathrm{C}_{m}$, then $\alpha=2 \varepsilon_{1}$ and $\alpha-\alpha_{i} \notin \Phi$ for $i>1$, so that $\Lambda\left(h_{\alpha_{i}}\right)=0$ for $i \neq 1$. (We are assuming the standard indexing of the simple roots as in 4.2.) Thus, $\Lambda=a \varpi_{1}$ and $\alpha_{j}=\alpha_{1}$. But since $(\alpha, \alpha)=2\left(\alpha_{1}, \alpha_{1}\right), k_{1}=2$, and $\Lambda\left(h_{\alpha}\right)=1$, we have using (2.1) that

$$
1=\Lambda\left(h_{\alpha}\right)=a\left\langle\varpi_{1}, \alpha\right\rangle=a \frac{2\left(\varpi_{1}, \alpha\right)}{(\alpha, \alpha)}=a \frac{4\left(\varpi_{1}, \alpha_{1}\right)}{2\left(\alpha_{1}, \alpha_{1}\right)}=a\left\langle\varpi_{1}, \alpha_{1}\right\rangle=a,
$$

so we can conclude that $\Lambda=\varpi_{1}$.

For $\Phi=\mathrm{A}_{m}$, we have $\alpha=\varepsilon_{1}-\varepsilon_{m+1}=\alpha_{1}+\cdots+\alpha_{m} ; \alpha-\alpha_{1}, \alpha-\alpha_{m} \in \Phi$; $\alpha-2 \alpha_{1} \notin \Phi, \alpha-2 \alpha_{m} \notin \Phi$; and $\alpha-\alpha_{i} \notin \Phi$ for $i \in\{2, \ldots, m-1\}$. This forces $\Lambda=a_{1} \varpi_{1}+a_{m} \varpi_{m}$. However, since $1=\Lambda\left(h_{\alpha}\right)=a_{1}+a_{m}$, we see that $\Lambda=\varpi_{1}$ or $\Lambda=\varpi_{m}$.

The proof of Theorem 4.4 in the case that $\alpha \in \Phi^{+}$will be complete once we show that $\alpha$ is a highest root of $\Phi$. Let $\widetilde{\mathfrak{g}}$ denote the graded subalgebra of $\mathfrak{g}$ generated by $f^{\Lambda}, \mathfrak{g}_{0}$, and $e^{\Gamma}$. Set $\widetilde{\mathfrak{g}}_{i}=\mathfrak{g}_{i} \cap \widetilde{\mathfrak{g}}$. Let $J$ be the sum of all graded ideals of $\widetilde{\mathfrak{g}}$ having zero intersection with the local part $\widetilde{\mathfrak{g}}_{-1} \oplus \mathfrak{g}_{0} \oplus \widetilde{\mathfrak{g}}_{1}$, and set $\overline{\mathfrak{g}}=\tilde{\mathfrak{g}} / J$. Clearly $\overline{\mathfrak{g}}=\bigoplus_{i=-q^{\prime}}^{r^{\prime}} \overline{\mathfrak{g}}_{i}$ is a graded Lie algebra satisfying all the hypotheses of Theorem 4.4. Indeed, we can identify $\overline{\mathfrak{g}}_{0}$ with $\mathfrak{g}_{0}$ and $\overline{\mathfrak{g}}_{ \pm 1}$ with $\mathfrak{g}_{ \pm 1}$, as $J$ intersects the local part trivially. The ideals $\mathcal{A}^{-}$and $\mathcal{A}^{+}$ (see Proposition 1.39) must be contained in $J$. As a result, the following conditions hold:

(a) If $\left[\bar{x}, \overline{\mathfrak{g}}_{-1}\right]=0$ for some $\bar{x} \in \overline{\mathfrak{g}}_{i}, i \geq 0$, then $\bar{x}=0$.

(b) If $\left[\bar{x}, \overline{\mathfrak{g}}_{1}\right]=0$ for some $\bar{x} \in \overline{\mathfrak{g}}_{i}, i \leq 0$, then $\bar{x}=0$.

Thus, in what follows we will assume that $\mathfrak{g}=\overline{\mathfrak{g}}$, and hence that $\mathfrak{g}$ is generated by $\mathfrak{g}_{0}, f^{\Lambda}, e^{\Gamma}$, that $\mathfrak{g}$ is transitive and 1-transitive (but not necessarily irreducible), and that there exists $\beta \in \Delta$ for which $\left[e_{\alpha}, e_{\beta}\right] \neq 0$ (as otherwise $\alpha$ is a highest root, and we are done). Again we suppose that $\Phi^{[k]}$ is the irreducible component of the root system $\Phi$ containing $\alpha$. As before, applying Lemma 4.3 to the weight vectors $f^{\Lambda}, e^{\Gamma}$ and $e_{-\alpha}$ we get 
$\Lambda\left(h_{\alpha}\right)=0$ or $\Lambda\left(h_{\alpha}\right)=1$. In either case, $\Gamma\left(h_{\alpha}\right)=-(\Lambda+\alpha)\left(h_{\alpha}\right) \neq 0$ since $p>3$.

Put $V_{-1}=\mathfrak{U}\left(\mathfrak{n}^{-}\right) f^{\Lambda}$ and $V_{1}=\mathfrak{U}\left(\mathfrak{n}^{+}\right) e^{\Gamma}$. For $i \geq 2$, define $V_{ \pm i}$ inductively by setting $V_{ \pm i}=\left[V_{ \pm 1}, V_{ \pm(i-1)}\right]$. Each $V_{i}$ is a $\mathfrak{g}_{0}$-submodule of $\mathfrak{g}_{i}$, and the graded subspace $I:=\mathfrak{g}_{0} \oplus \sum_{i \neq 0} V_{i}$ is invariant under the endomorphisms ad $f^{\Lambda}$ and $\operatorname{ad} e^{\Gamma}$. Therefore, $I$ is an ideal of $\mathfrak{g}$ containing $f^{\Lambda}, e^{\Gamma}$, and $\mathfrak{g}_{0}$. But then $I=\mathfrak{g}$, yielding $\mathfrak{g}_{-1}=\mathfrak{U}\left(\mathfrak{n}^{-}\right) f^{\Lambda}$.

Set $f^{\Lambda^{\prime}}=\left[\left[e_{-\beta}, f^{\Lambda}\right], f^{\Lambda}\right]$ and $e^{\Gamma^{\prime}}=\left[\left[e_{\alpha}, e^{\Gamma}\right], e^{\Gamma}\right]$, and let $F^{\Lambda}=\operatorname{ad} f^{\Lambda}$ and $E_{-\beta}=\operatorname{ad} e_{-\beta}$ denote the adjoint mappings. Then since $\beta+\alpha$ is a root, and twice a root is never a root, we must have $\beta \neq \alpha$ and

$$
\begin{aligned}
{\left[f^{\Lambda^{\prime}}, e^{\Gamma^{\prime}}\right] } & =\left(\left(F^{\Lambda}\right)^{2} E_{-\beta}-2 F^{\Lambda} E_{-\beta} F^{\Lambda}+E_{-\beta}\left(F^{\Lambda}\right)^{2}\right)\left(\left[\left[e_{\alpha}, e^{\Gamma}\right], e^{\Gamma}\right]\right) \\
& =E_{-\beta}\left(F^{\Lambda}\right)^{2}\left(\left[\left[e_{\alpha}, e^{\Gamma}\right], e^{\Gamma}\right]\right) \\
& =2 E_{-\beta} F^{\Lambda}\left(\left[\left[e_{\alpha}, e_{-\alpha}\right], e^{\Gamma}\right]\right)=2 \Gamma\left(h_{\alpha}\right)\left[e_{-\beta}, e_{-\alpha}\right] .
\end{aligned}
$$

As $\beta \in \Delta$, it can be easily checked that $f^{\Lambda^{\prime}} \in \mathfrak{g}_{-2}$ is a $\mathfrak{b}^{+}$-primitive vector of weight $\Lambda^{\prime}$. We claim that $e^{\Gamma^{\prime}} \in \mathfrak{g}_{2}$ is a $\mathfrak{b}^{-}$-primitive vector of weight $\Gamma^{\prime}$. Indeed, if this were not true, then there would be a sequence $F_{i_{1}}, \ldots, F_{i_{n}}$ of length $n \geq 1$, where $F_{i}=\operatorname{ad} e_{-\alpha_{i}}$, such that $e^{\prime}:=F_{i_{1}} \cdots F_{i_{n}} e^{\Gamma^{\prime}} \neq 0$ and $\left[\mathfrak{n}^{-}, e^{\prime}\right]=0$. But

$$
e^{\prime}=\left[\left[F_{i_{1}} \cdots F_{i_{n}} e_{\alpha}, e^{\Gamma}\right], e^{\Gamma}\right]
$$

implies $0 \neq e^{\prime}=\xi\left[\left[e_{\sigma}, e^{\Gamma}\right], e^{\Gamma}\right]$ for some $\xi \in \mathbb{F}^{\times}$and $\sigma \in \Phi^{+}$. Since $n \geq 1$, we must have

$$
\left[f^{\Lambda}, e^{\prime}\right]=2 \xi\left[\left[e_{\sigma}, e_{-\alpha}\right], e^{\Gamma}\right]=0 .
$$

Since $\mathfrak{g}_{-1}=\mathfrak{U}\left(\mathfrak{n}^{-}\right) f^{\Lambda}$, this yields $\left[e^{\prime}, \mathfrak{g}_{-1}\right]=0$, contradicting transitivity and proving the claim.

Next we demonstrate that $(2 \Lambda-\beta)\left(\mathfrak{t}^{[k]}\right) \neq 0$. Assume that $\theta$ is any positive root for which $\left[e_{-\alpha}, e_{-\theta}\right] \neq 0$. Set

$$
x_{\lambda}=\left[e_{-\theta}, f^{\Lambda}\right], \quad x_{\gamma}=e^{\Gamma}, \quad e_{-\delta}=\left[e_{-\alpha}, e_{-\theta}\right] .
$$

An easy calculation shows that these weight vectors satisfy all the conditions of Lemma 4.3. Therefore, $(\Lambda-\theta)\left(h_{\alpha+\theta}\right) \in\{0,1\}$. Setting $\theta=\beta$, we get $(\Lambda-\beta)\left(h_{\alpha+\beta}\right) \in\{0,1\}$. If $(2 \Lambda-\beta)\left(\mathfrak{t}^{[k]}\right)=0$, then in particular $\frac{1}{2} \beta\left(h_{\alpha}\right)=$ $\Lambda\left(h_{\alpha}\right) \in\{0,1\}$ and $1=\frac{1}{2} \beta\left(h_{\beta}\right)=\Lambda\left(h_{\beta}\right)$. If $(\alpha, \beta)=0$, then

$$
\begin{aligned}
(\Lambda-\beta)\left(h_{\alpha+\beta}\right) & =-\frac{1}{2} \beta\left(h_{\alpha+\beta}\right) \\
& =-\frac{(\beta, \alpha+\beta)}{(\alpha+\beta, \alpha+\beta)}=-\frac{(\beta, \beta)}{(\alpha+\beta, \alpha+\beta)} \in\{0,1\} .
\end{aligned}
$$

This forces $(\alpha+\beta, \alpha+\beta)=-(\beta, \beta)$, contradicting the assumption $p>3$. Hence it must be that $(\alpha, \beta) \neq 0$ and $\Lambda\left(h_{\alpha}\right)=\frac{1}{2} \beta\left(h_{\alpha}\right)=1$. This implies 
that $(\beta, \alpha)=(\alpha, \alpha)$ so that $(\beta, \alpha+\beta)=(\alpha, \alpha)+(\beta, \beta) \neq 0$. Consequently, $(\Lambda-\beta)\left(h_{\alpha+\beta}\right)=-\frac{(\beta, \alpha+\beta)}{(\alpha+\beta, \alpha+\beta)}=1$. This means that $2(\alpha, \alpha)+(\beta, \beta) \equiv 0$ $\bmod p$. If $\alpha$ is a long root, then $\beta\left(h_{\alpha}\right) \in\{-1,0,1\}$ (see [Bou1]). However, we know that $\beta\left(h_{\alpha}\right)=2$. As $p>3$, we conclude $\alpha$ is a short root. Then from the relation $2(\alpha, \alpha)+(\beta, \beta) \equiv 0$ it follows that only the case that $p=5$ and $\Phi^{[k]}$ is of type $\mathrm{G}_{2}$ is possible. We assume that the elements of the base $\left\{\alpha_{1}, \alpha_{2}\right\}$ of $\mathrm{G}_{2}$ are numbered so $\alpha_{1}$ is short and $\alpha_{2}$ is long. Since $\beta$ is simple and long, it must be that $\beta=\alpha_{2}$. Then from the fact that $\alpha$ is a short positive root, and $\alpha+\beta \in \Phi^{[k]}$, we see that $\alpha=\alpha_{1}$. Set $\theta=\alpha_{1}+\alpha_{2}$. Then $\left[e_{-\alpha}, e_{-\theta}\right] \neq 0, \Lambda\left(h_{\alpha+\theta}\right)=\frac{1}{2} \alpha_{2}\left(h_{2 \alpha_{1}+\alpha_{2}}\right)=0$, and $\theta\left(h_{\alpha+\theta}\right)=\left(\alpha_{1}+\alpha_{2}\right)\left(h_{2 \alpha_{1}+\alpha_{2}}\right)=1$. Therefore, $(\Lambda-\theta)\left(h_{\alpha+\theta}\right)=-1 \notin\{0,1\}$.

Thus, we have proved that $(2 \Lambda-\beta)\left(\mathfrak{t}^{[k]}\right) \neq 0$, that $f^{\Lambda^{\prime}}$ is a $\mathfrak{b}^{+}$-primitive vector, and that $e^{\Gamma^{\prime}}$ is a $\mathfrak{b}^{-}$-primitive vector. Hence, after scaling $e^{\Gamma^{\prime}}$ by a suitable scalar factor if necessary so that $\left[f^{\Lambda^{\prime}}, e^{\Gamma^{\prime}}\right]=e_{-\alpha-\beta}$, we have that the triple $\left(f^{\Lambda^{\prime}}, \mathfrak{g}_{0}, e^{\Gamma^{\prime}}\right)$ satisfies all the conditions of Theorem 4.4. Repeating the argument with this new triple, we will eventually arrive at the highest root of $\Phi^{[k]}$. Thus, we may assume that $\alpha+\beta$ is the highest root of $\Phi^{[k]}$, and that $\left[f^{\Lambda^{\prime}}, e^{\Gamma^{\prime}}\right]=e_{-\alpha-\beta}$. Applying the previous part of the proof to the triple $\left(f^{\Lambda^{\prime}}, \mathfrak{g}_{0}, e^{\Gamma^{\prime}}\right)$, we obtain that $\Phi=\Phi^{[k]}$, and either $\Phi=\mathrm{A}_{m}, \Lambda^{\prime} \in\left\{\varpi_{1}, \varpi_{m}\right\}$, or else $\Phi=\mathrm{C}_{m}, \Lambda^{\prime}=\varpi_{1}$.

We adopt the notation in (4.1) and (4.2). Let $\Phi=\mathrm{C}_{m}, m \geq 2$. Then $\alpha+\beta=2 \varepsilon_{1}$, and since $\beta \in \Delta, \beta=\varepsilon_{1}-\varepsilon_{2}$ and $\alpha=\varepsilon_{1}+\varepsilon_{2}$. Because $\Lambda^{\prime}=$ $2 \Lambda-\beta=\varpi_{1}=\varepsilon_{1}$ in this case, we obtain $2 \Lambda\left(h_{\alpha}\right)=1$. But $\Lambda\left(h_{\alpha}\right) \in\{0,1\}$ by our previous considerations. This shows that the case $\Phi=\mathrm{C}_{m}$ cannot occur; i.e., there cannot be any such $\beta$, so that $\alpha$ must be the highest root.

Now suppose that $\Phi=\mathrm{A}_{m}$ and $\Lambda^{\prime}=2 \Lambda-\beta=\varpi_{1}=\varepsilon_{1}$. Since we are assuming $\alpha \in \Phi^{+}$is not the highest root, we have $m \geq 2$. As $\alpha+\beta=$ $\alpha_{1}+\cdots+\alpha_{m}, \beta \in \Delta$, and $\alpha \in \Phi$, it must be $\beta=\alpha_{1}$ or $\beta=\alpha_{m}$. Then $\Lambda\left(h_{\alpha}\right) \in\{0,1\}$ forces $\beta=\alpha_{m}, \Lambda\left(h_{\alpha+\beta}\right)=1$, and $\Lambda\left(h_{\alpha}\right)=0$. Set

$$
\begin{gathered}
x_{\lambda^{\prime}}=\left[\left[\left[e_{-\beta}, f^{\Lambda}\right], f^{\Lambda}\right], f^{\Lambda}\right], \quad x_{\gamma^{\prime}}=\left[\left[\left[\left[e^{\Gamma}, e_{\alpha}\right], e_{\alpha}\right], e^{\Gamma}\right], e^{\Gamma}\right], \\
e_{-\delta^{\prime}}=-8\left[e_{-\beta}, e_{-\alpha}\right] .
\end{gathered}
$$

Then

$$
\begin{aligned}
{\left[x_{\lambda^{\prime}}, x_{\gamma^{\prime}}\right] } & =\left(\sum_{j=0}^{3}(-1)^{j}\left(\begin{array}{l}
3 \\
j
\end{array}\right)\left(F^{\Lambda}\right)^{j} E_{-\beta}\left(F^{\Lambda}\right)^{3-j}\right)\left(\left[\left[\left[\left[e^{\Gamma}, e_{\alpha}\right], e_{\alpha}\right], e^{\Gamma}\right], e^{\Gamma}\right]\right) \\
& =E_{-\beta}\left(F^{\Lambda}\right)^{3}\left(\left[\left[\left[\left[e^{\Gamma}, e_{\alpha}\right], e_{\alpha}\right], e^{\Gamma}\right], e^{\Gamma}\right]\right) \\
& =E_{-\beta}\left(F^{\Lambda}\right)^{2}\left(\left[\left[\left[\left[e_{-\alpha}, e_{\alpha}\right], e_{\alpha}\right], e^{\Gamma}\right], e^{\Gamma}\right]+2\left[\left[\left[\left[e^{\Gamma}, e_{\alpha}\right], e_{\alpha}\right], e_{-\alpha}\right], e^{\Gamma}\right]\right) \\
& =(-4 \Gamma-3 \alpha)\left(h_{\alpha}\right) E_{-\beta}\left(F^{\Lambda}\right)^{2}\left(\left[\left[e_{\alpha}, e^{\Gamma}\right], e^{\Gamma}\right]\right) \\
& =(2 \Gamma)\left(h_{\alpha}\right)(4 \Lambda+\alpha)\left(h_{\alpha}\right)\left[e_{-\beta}, e_{-\alpha}\right]=-8\left[e_{-\beta}, e_{-\alpha}\right],
\end{aligned}
$$


because $\Lambda+\Gamma=-\alpha$ and $\Lambda\left(h_{\alpha}\right)=0$. The relations $\left[x_{\lambda^{\prime}}, e_{\delta^{\prime}}\right]=\left[x_{\gamma^{\prime}}, e_{-\delta^{\prime}}\right]=0$ are easy to verify. Applying Lemma 4.3, we get that $(3 \Lambda-\beta)\left(h_{\alpha+\beta}\right) \in\{0,1\}$. But it follows from the above calculation that $(3 \Lambda-\beta)\left(h_{\alpha+\beta}\right)=2$. This shows that the case we are considering $\left(\Phi=\mathrm{A}_{m}, \Lambda^{\prime}=\varpi_{1}\right)$ cannot occur. The case $\Phi=\mathrm{A}_{m}, m \geq 2, \Lambda^{\prime}=\varpi_{m}$ can be handled similarly. This completes the proof of Theorem 4.4.

\subsection{Additional assumptions}

In the remainder of the chapter, we will often impose some or all of the following conditions in addition to our blanket assumptions (1)-(2).

(3) $\mathfrak{g}_{-1}$ is an irreducible $\mathfrak{g}_{0}$-module;

(4) $\mathfrak{g}$ is transitive $(1.2)$;

(5) $\mathfrak{g}$ is 1 -transitive (1.3);

(6) $\mathfrak{g}_{1}$ is a faithful $\mathfrak{g}_{0}$-module.

REMARK 4.6. When $\mathfrak{g}$ is a graded Lie algebra satisfying constraints (1)(4) along with $(6)$, then $\left[\mathfrak{g}_{1}, \mathfrak{g}_{-1}\right] \neq 0$ by transitivity. Since $\mathfrak{g}_{0}$ is assumed to act faithfully on $\mathfrak{g}_{1}$, we have $\left[\left[\mathfrak{g}_{1}, \mathfrak{g}_{-1}\right], \mathfrak{g}_{1}\right] \neq 0$. Thus all the hypotheses of Theorem 1.63 hold, so $\mathfrak{g}_{-1}$ is a restricted $\mathfrak{g}_{0}^{(1)}$-module. Condition (5) (1transitivity) implies (6), so whenever constraints (1)-(5) are assumed (as in the statement of the Main Theorem, for example), $\mathfrak{g}_{-1}$ must be a restricted $\mathfrak{g}_{0}^{(1)}$-module.

\subsection{Computing weights of $\mathfrak{b}^{-}$-primitive vectors in $\mathfrak{g}_{1}$}

In Remark 4.5, we have argued that when $\mathfrak{g}$ is transitive and $\mathfrak{g}_{-1}$ is irreducible, one of the hypotheses of Theorem 4.4 holds automatically-namely, $\Lambda\left(\mathfrak{t}{ }^{[i]}\right) \neq 0$ for all $i$ whenever $\Lambda$ is the weight of $\mathfrak{a}^{+}$-primitive vector of $\mathfrak{g}_{-1}$. Here we show that under a few additional assumptions on the $\mathfrak{g}_{0}$-module $\mathfrak{g}_{-1}$, the other assumption in Theorem 4.4 holds as well; $\Gamma\left(\mathfrak{t}^{[i]}\right) \neq 0$ for all $i$ whenever $\Gamma$ is the weight of a $\mathfrak{b}^{-}$-primitive vector of $\mathfrak{g}_{1}$.

THEOREM 4.7. Let $\mathfrak{g}$ be a graded Lie algebra satisfying conditions (1)(4), and assume $\mathfrak{g}_{-1}$ is a restricted $\mathfrak{g}_{0}^{(1)}$-module with a $\mathfrak{b}^{+}$-primitive vector of weight $\Lambda$. Suppose also that $\mathfrak{g}_{1}$ contains an irreducible $\mathfrak{g}_{0}$-submodule generated by a $\mathfrak{b}^{-}$-primitive vector $e^{-\Lambda}$ corresponding to the weight $-\Lambda \in \mathfrak{t}^{*}$. Then the following hold:

(i) $\Gamma\left(\mathfrak{t}^{[i]}\right) \neq 0, i=1, \ldots, \ell$ whenever $\mathfrak{g}_{1}$ has a $\mathfrak{b}^{-}$-primitive vector of weight $\Gamma$;

(ii) If all $\mathfrak{b}^{-}$-primitive vectors of $\mathfrak{g}_{1}$ have weight $-\Lambda$, then $\mathfrak{g}_{1}$ is an irreducible $\mathfrak{g}_{0}^{(1)}$-module. 
Proof. Given a linear function $\psi$ on $\mathfrak{t}$ we denote by $\psi^{[i]}$ the restriction of $\psi$ to $\mathfrak{t}^{[i]} \cap \mathfrak{g}_{0}^{(1)}$. Assume $e^{\Gamma} \in \mathfrak{g}_{1}$ is a $\mathfrak{b}^{-}$-primitive vector of weight $\Gamma$ with $\Gamma\left(\mathfrak{t}^{[k]}\right)=0$ for some $k$. As $\mathfrak{g}$ is transitive, we can regard $\mathfrak{g}_{1}$ as a submodule of $\operatorname{Hom}\left(\mathfrak{g}_{-1}, \mathfrak{g}_{0}\right) \cong \mathfrak{g}_{-1}^{*} \otimes \mathfrak{g}_{0}$. In particular, $\mathfrak{g}_{1}$ is a restricted $\mathfrak{g}_{0}^{(1)}$-module. Let $f^{\Lambda}$ be a $\mathfrak{b}^{+}$-primitive vector of $\mathfrak{g}_{-1}$ corresponding to the weight $\Lambda$. By transitivity and irreducibility, we have from Remark 4.5 that $\Lambda\left(\mathfrak{t}^{[i]}\right) \neq 0$ for any $i$, so $\Gamma \neq-\Lambda$. Then by transitivity, $\left[f^{\Lambda}, e^{\Gamma}\right]=\zeta e_{-\alpha}$ for some $\zeta \in \mathbb{F}^{\times}$ and some $\alpha \in \Phi$. It follows that $\Lambda^{[k]}$ is a root of $\left(\mathfrak{g}_{0}^{[k]}\right)^{(1)}$.

According to Theorem 3.21, the subalgebra $\widetilde{\mathfrak{g}}$ generated by $f^{\Lambda}, \mathfrak{g}_{0}$, and $e^{-\Lambda}$ modulo its Weisfeiler radical $\mathcal{M}(\widetilde{\mathfrak{g}})$ is isomorphic to a classical Lie algebra with a standard grading. Let $\widetilde{\Delta}=\Delta \cup\{-\Lambda\}$ be the canonical base for the root system of the classical Lie algebra $\widetilde{\mathfrak{g}} / \mathcal{M}(\widetilde{\mathfrak{g}})$. Using the Dynkin diagrams in $\left[\right.$ Bou1], it is straightforward to see that $\Lambda^{[i]}$ is a minuscule weight (see Section 2.2) of the root system $\Phi^{[i]}$ except in the following cases:

(I) $\Phi=\Phi^{[1]} \cup \Phi^{[2]}$ where $\Phi^{[1]}=\mathrm{A}_{m-1}, \Lambda^{[1]}=\varpi_{m-1}^{[1]}, \Phi^{[2]}=\mathrm{A}_{1}, \Lambda^{[2]}=$ $2 \varpi_{1}^{[2]}$, and the resulting root system is of type $\mathrm{B}_{m+1}$,

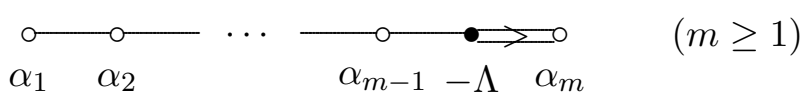

(II) $\Phi=\Phi^{[1]}=\mathrm{A}_{m}, \Lambda^{[1]}=2 \varpi_{m}$, and the resulting root system is of type $\mathrm{C}_{m+1}$,

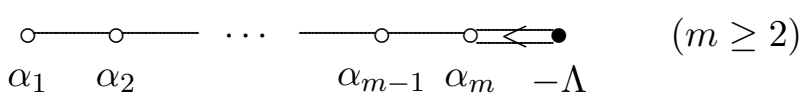

(III) $\Phi=\Phi^{[1]} \cup \Phi^{[2]}$ where $\Phi^{[1]}=\mathrm{A}_{1}, \Lambda^{[1]}=\varpi_{1}^{[1]}, \Phi^{[2]}=\mathrm{A}_{2}, \Lambda^{[2]}=2 \varpi_{1}^{[2]}$, and the resulting root system is of type $\mathrm{F}_{4}$,

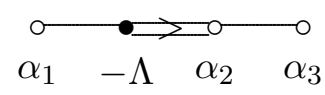

(IV) $\Phi=\Phi^{[1]}=\mathrm{A}_{1}, \Lambda^{[1]}=3 \varpi_{1}$, and the resulting root system is of type $\mathrm{G}_{2}$,

$$
\stackrel{\circ}{\alpha_{1} \quad-\Lambda}
$$

(V) $\Phi=\Phi^{[1]}=\mathrm{C}_{3}, \Lambda^{[1]}=\varpi_{3}$, and the resulting root system is of type $\mathrm{F}_{4}$, 


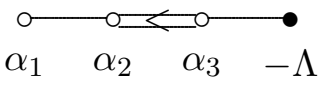

If $\Lambda^{[k]}$ is a minuscule weight, then $\Lambda^{[k]}\left(h_{\gamma}\right) \in\{-1,0,1\}$ for all $\gamma \in \Phi^{[k]}$. Since $\gamma\left(h_{\gamma}\right)=2$, this implies that $\Lambda^{[k]}$ it is not a root of $\left(\mathfrak{g}_{0}^{[k]}\right)^{(1)}$, contrary to our earlier remarks. Thus, $\Lambda^{[k]}$ is not minuscule, and hence it remains to handle Cases (I)-(V).

We can rule out Case (II), since here $\Phi=\Phi^{[1]}=\mathrm{A}_{m}$ and $\Lambda^{[1]}=2 \varpi_{m}$ is not a root of $\left(\mathfrak{g}^{[1]}\right)^{(1)}$ for $m \geq 2$. In Case (III), $\Phi=\Phi^{[1]} \cup \Phi^{[2]}$, where $\Phi^{[1]}=$ $\mathrm{A}_{1}$ and $\Phi^{[2]}=\mathrm{A}_{2}$, and $\Lambda^{[1]}=\varpi_{1}^{[1]}, \Lambda^{[2]}=2 \varpi_{1}^{[2]}$. Hence, $\Lambda^{[i]}$ is not a root of $\left(\mathfrak{g}^{[i]}\right)^{(1)}$ for $i=1,2$. Likewise in Case $(\mathrm{V}), \Phi=\Phi^{[1]}=\mathrm{C}_{3}$, and $\varpi_{3}$ is not a root of $\left(\mathfrak{g}^{[1]}\right)^{(1)}$.

In Case (I), if $m \geq 2$, then $\Phi=\Phi^{[1]} \cup \Phi^{[2]}$, where $\Phi^{[1]}=\mathrm{A}_{m-1}, \Phi^{[2]}=$ $\mathrm{A}_{1}, \quad \Lambda^{[1]}=\varpi_{m-1}^{[1]}$, and $\Lambda^{[2]}=2 \varpi_{1}^{[2]}$. If the root $\alpha$ in the first paragraph of the proof belongs to $\Phi^{+}$, then since $\Lambda^{[i]} \neq 0$ for $i=1,2$, Theorem 4.4 applies and gives that $\Phi$ is irreducible. Since this is false, we must have $\alpha \in \Phi^{-}$. If $\Gamma\left(\mathfrak{t}^{[1]}\right)=0$, then $\Lambda^{[1]}$ is a root of $\left(\mathfrak{g}^{[1]}\right)^{(1)}$, contradicting the fact that $\Lambda^{[1]}$ is a minuscule weight for $\Phi^{[1]}$. Hence, we may assume $\Gamma\left(\mathfrak{t}^{[2]}\right)=0$, so that $\alpha=-\alpha_{1}^{[2]}$. (It is easy to check that $\alpha=-\alpha_{1}^{[2]}$ also holds when $m=1$.) But then

$$
\left[e^{\Gamma},\left[f^{\Lambda},\left[f^{\Lambda}, e_{\alpha}\right]\right]\right]=-2 \zeta\left[f^{\Lambda},\left[e_{-\alpha}, e_{\alpha}\right]\right]=4 \zeta f^{\Lambda} \neq 0,
$$

where $\zeta$ is as in the first paragraph of the proof, which implies $\left[f^{\Lambda},\left[f^{\Lambda}, e_{\alpha}\right]\right] \neq$ 0 . On the other hand, $f^{\Lambda}$ corresponds to a long root of $\widetilde{\mathfrak{g}}_{0}$, and $\left(\operatorname{ad} f^{\Lambda}\right)^{2}\left(\widetilde{\mathfrak{g}}_{0}\right)=$ $\mathbb{F} f^{\Lambda} \subseteq \mathfrak{g}_{-1}$. As $\left[\left[f^{\Lambda},\left[f^{\Lambda}, e_{\alpha}\right]\right] \in \mathfrak{g}_{-2}\right.$, this shows that Case (I) cannot occur.

In Case (IV), we have $\Phi=\Phi^{[1]}=\mathrm{A}_{1}, \Lambda^{[1]}=3 \varpi_{1}$, and the resulting root system is of type $\mathrm{G}_{2}$. Then $\alpha= \pm \alpha_{1}$. If $\alpha=\alpha_{1}$, then Theorem 4.4 gives $\Lambda=\varpi_{1}$ (the highest weight of the natural module), which is false. Hence $\alpha=-\alpha_{1}$. But in this case, $\Gamma^{[1]}=\alpha_{1}-\Lambda=-\varpi_{1}$, and $\Gamma(\mathfrak{t}) \neq 0$. This completes the proof of part (i).

From now on we assume that all $\mathfrak{b}^{-}$-primitive vectors of $\mathfrak{g}_{1}$ have weight $-\Lambda$. Set $L=\widetilde{\mathfrak{g}} / \mathcal{M}(\widetilde{\mathfrak{g}})$. It follows from Theorem 3.21 that $\widetilde{G}:=\operatorname{Aut}(L)^{\circ}$ is a simple algebraic group (of adjoint type), and ad $L \cong L$ is a $\widetilde{G}$-stable ideal of codimension $\leq 1$ in $\operatorname{Lie}(\widetilde{G})=\operatorname{Der}(L)$ (the equality $\operatorname{Lie}(\widetilde{G})=L$ holds if and only if $L \neq \mathfrak{p s l}_{k p}$ ). There exists a homomorphism of algebraic groups $\phi: \mathbb{F}^{\times} \rightarrow \widetilde{G}$ such that for all $i \in \mathbb{Z}$ we have

$$
L_{i}=(\widetilde{\mathfrak{g}} / \mathcal{M}(\widetilde{\mathfrak{g}}))_{i}=\left\{x \in L \mid \phi(t)(x)=t^{i} x \text { for all } t \in \mathbb{F}^{\times}\right\}
$$


(see the proof of Theorem 3.21 for more detail). Let $\widetilde{G}_{0}$ be the centralizer in $\widetilde{G}$ of the one-dimensional torus $\phi\left(\mathbb{F}^{\times}\right)$. This is a connected, reductive subgroup of $\widetilde{G}_{0}$, and it is immediate from [Bo, Sec. 9.4] that $\operatorname{Lie}\left(\widetilde{G}_{0}\right)$ consists of all derivations of $L$ commuting with $\phi\left(\mathbb{F}^{\times}\right)$. Since $\phi\left(\mathbb{F}^{\times}\right)$acts trivially on $\operatorname{Lie}(\widetilde{G}) / \operatorname{ad} L$, the Lie algebra $\mathfrak{g}_{0} \cong(\widetilde{\mathfrak{g}} / \mathcal{M}(\widetilde{\mathfrak{g}}))_{0} \cong \operatorname{ad}_{L}\left(L_{0}\right)$ can be identified with a $\widetilde{G}_{0}$-stable ideal of codimension $\leq 1$ in $\operatorname{Lie}\left(\widetilde{G}_{0}\right)$. Moreover, $\left(\operatorname{Lie}\left(\widetilde{G}_{0}\right)\right)^{(1)} \cong \mathfrak{g}_{0}^{(1)}$ as Lie algebras. Identifying the $\mathfrak{g}_{0}$-module $L_{-1}$ with $\mathfrak{g}_{-1}$, we see that the adjoint action of $\mathfrak{g}_{0}$ on $\mathfrak{g}_{0}$ and $\mathfrak{g}_{-1}$ is induced by the differential of the (rational) action of $\widetilde{G}_{0}$ on $L$. There exist maximal unipotent subgroups $N^{ \pm}$and a maximal torus $\widetilde{T}$ in $\widetilde{G}_{0}$ such that $\operatorname{Lie}\left(N^{ \pm}\right)=\mathfrak{n}^{ \pm}$and $\operatorname{Lie}(\widetilde{T}) \supseteq \mathfrak{t}$.

Let $G_{0}$ be the derived subgroup of $\widetilde{G}_{0}$, and set $T=\widetilde{T} \cap G_{0}$. It follows from the general theory of algebraic groups that $G_{0}$ is semisimple (but not necessarily simply connected), that $T$ is a maximal torus of $G_{0}$, and that $\widetilde{G}_{0}=G_{0} \cdot Z\left(\widetilde{G}_{0}\right)^{\circ}$; see $[\mathbf{B o}]$, for example. Note that $N^{ \pm} \subset G_{0}$, and $\mathfrak{g}_{0}^{(1)}=$ $\left(\operatorname{Lie}\left(\widetilde{G}_{0}\right)\right)^{(1)}=\left(\operatorname{Lie}\left(G_{0}\right)\right)^{(1)}$. Let $\widehat{G}_{0}$ be the simply connected cover of $G_{0}$. It is well-known that there exists a surjective homomorphism of algebraic groups $\iota: \widehat{G}_{0} \rightarrow G_{0}$ whose kernel is finite (and central in $\widehat{G}_{0}$ ) and whose restriction to any maximal unipotent subgroup $\widehat{U}$ of $\widehat{G}_{0}$ induces an isomorphism of algebraic groups $\widehat{U} \stackrel{\sim}{\rightarrow} \iota(\widehat{U})$. It is straightforward to see that $\operatorname{ker}(\mathrm{d} \iota)_{e} \subseteq$ $\mathfrak{Z}\left(\operatorname{Lie}\left(G_{0}\right)\right)$. The inverse image $\widehat{T}:=\iota^{-1}(T)$ is a maximal torus of $\widehat{G}_{0}$. Since $\widehat{G}_{0}$ is simply connected, the Lie algebra Lie $\left(\widehat{G}_{0}\right)$ is generated by root vectors relative to $\widehat{T}$ ( this is due to the fact that $\widehat{T}$ has the smallest possible group of rational cocharacters). As $\mathfrak{s l}_{2}$ is simple for $p>2$, we then have $\left(\operatorname{Lie}\left(\widehat{G}_{0}\right)\right)^{(1)}=\operatorname{Lie}\left(\widehat{G}_{0}\right)$. In conjunction with our earlier remarks, this shows that $(\mathrm{d} \iota)_{e}$ maps $\operatorname{Lie}(\widehat{G})$ onto $\mathfrak{g}_{0}^{(1)}$. As a consequence, the adjoint action of $\mathfrak{g}_{0}^{(1)}$ on $\mathfrak{g}_{-1}$ and $\mathfrak{g}_{0}$ is induced by the differential of a rational action on $\mathfrak{g}_{-1} \oplus \mathfrak{g}_{0}$ of the semisimple, simply connected group $\widehat{G}_{0}$. We retain our notation associated with the lattice of weights of $\widehat{T}$.

To prove that $\mathfrak{g}_{1}$ is irreducible, we identify it with a $\mathfrak{g}_{0}^{(1)}$-submodule of $\operatorname{Hom}\left(\mathfrak{g}_{-1}, \mathfrak{g}_{0}\right)$. By the preceding remark, the action of $\mathfrak{g}_{0}$ on $\operatorname{Hom}\left(\mathfrak{g}_{-1}, \mathfrak{g}_{0}\right)$ $\cong \mathfrak{g}_{-1}^{*} \otimes \mathfrak{g}_{0}$ is induced by the differential of the natural rational action of $\widehat{G}_{0}$ on Hom $\left(\mathfrak{g}_{-1}, \mathfrak{g}_{0}\right)$. Since $\iota\left(\widehat{G}_{0}\right)=G_{0}$ and $\widetilde{G}_{0}=G_{0} \cdot Z(\widetilde{G})^{\circ}$, the $\widehat{G}_{0}$-module $\mathfrak{g}_{-1}$ is irreducible. We denote by $\lambda$ the maximal weight of the representation of $\widehat{G}_{0}$ on $\mathfrak{g}_{-1}$. By the above discussion, $(\mathrm{d} \iota)_{e}$ sends $\operatorname{Lie}(\widehat{T})$ onto $\mathfrak{t} \cap \mathfrak{g}_{0}^{(1)}$. To simplify notation we will identify $\mathfrak{t} \cap \mathfrak{g}_{0}^{(1)}$ with $\operatorname{Lie}(\widehat{T}) / \operatorname{ker}(\mathrm{d} \iota)_{e}$. As $\widehat{G}_{0}$ is simply connected, the differential map $\mathrm{d}: X(\widehat{T}) \rightarrow \operatorname{Lie}(\widehat{T})^{*}, \quad \nu \mapsto(\mathrm{d} \nu)_{e}$, has kernel $p X(\widehat{T})$ and induces an isomorphism $(X(\widehat{T}) / p X(\widehat{T})) \otimes_{\mathbb{Z}} \mathbb{F} \stackrel{\sim}{\longrightarrow}$ $\operatorname{Lie}(\widehat{T})^{*}$. As $\mathrm{d} \lambda$ vanishes on $\operatorname{ker}(\mathrm{d} \iota)_{e}$, we may regard it as a linear function on $\mathfrak{t} \cap \mathfrak{g}_{0}^{(1)}$. Note that $\lambda \in X_{1}(\widehat{T})$, because $\mathfrak{g}_{-1}$ is an irreducible $\mathfrak{g}_{0}^{(1)}$-module (see 
Remark 1.70 and Proposition 2.13). Since $d \lambda$ is the weight of a $\mathfrak{b}^{+}$-primitive vector in the $\mathfrak{g}_{0}^{(1)}$-module $\mathfrak{g}_{-1}$, it must coincide with the restriction of $\Lambda$ to $\mathfrak{t} \cap \mathfrak{g}_{0}^{(1)}$.

Suppose $\Lambda^{[k]}$ is a minuscule weight for all $k \in\{1, \ldots, \ell\}$. Then $\lambda$ is a minuscule weight of $X(\widehat{T})$, which means that $X\left(\operatorname{Hom}\left(\mathfrak{g}_{-1}, \mathfrak{g}_{0}\right)\right)=X^{\prime} \cup X^{\prime \prime}$ where $X^{\prime}=\{-w \lambda \mid w \in W\}$ and $X^{\prime \prime}=\{-w \lambda+\gamma \mid w \in W, \gamma \in \Phi\}$ (here $W=N_{\widehat{G}_{0}}(T) / Z_{\widehat{G}_{0}}(T)$ is the Weyl group of $\left.\widehat{G}_{0}\right)$. As $\Phi$ does not have any components of type $\mathrm{G}_{2}$, this implies that $\frac{2(\nu, \gamma)}{(\gamma, \gamma)} \in\{0, \pm 1, \pm 2, \pm 3\}$ for any $\nu \in X^{\prime} \cup X^{\prime \prime}$ and $\gamma \in \Phi$. As $p>3$, it follows that all dominant weights of the $\widehat{G}_{0}$-module $\operatorname{Hom}\left(\mathfrak{g}_{-1}, \mathfrak{g}_{0}\right)$ belong to $X_{1}(\widehat{T})$. Hence Proposition 2.14 applies and we obtain that $\mathfrak{g}_{1}$ is $\widehat{G}_{0}$-stable.

Let $\mu$ be a minimal weight of the $\widehat{G}_{0}$-module $\mathfrak{g}_{1}$. Then $w_{0} \mu$ is a dominant weight of $\mathfrak{g}_{1}$, hence it belongs to $X_{1}(\widehat{T})$. It follows that $\mu \in-X_{1}(\widehat{T})$. As the weight component $\mathfrak{g}_{1}^{\mu}$ consists of $\mathfrak{b}^{-}$-primitive vectors, the weight $\mathrm{d} \mu$ coincides with the restriction of $-\Lambda$ to $\mathfrak{t} \cap \mathfrak{g}_{0}^{(1)}$. As $-\lambda \in-X_{1}(\widehat{T})$ too, it must be that $\mu=-\lambda$.

Let $\nu \in X\left(\mathfrak{g}_{1}\right)$. Then there exists $w \in W$ such that $w \nu \in-X(T)_{+}$. Because $X\left(\mathfrak{g}_{1}\right)$ is $W$-stable, and $-\lambda$ is the only minimal weight of $\mathfrak{g}_{1}$, we have that $w \nu \geq-\lambda$. But then $\lambda \geq-w \nu$ with $-w \nu \in X(T)_{+}$. As $\lambda$ is a minuscule weight, this implies $w \nu=-\lambda$. Hence all weights of $\mathfrak{g}_{1}$ are conjugate under $W$. If $e^{\mu}$ and $e^{-\Lambda}$ are linearly independent vectors of $\mathfrak{g}_{1}^{\mu}$, then $\left[f^{\Lambda},\left[t e^{-\Lambda}-e^{\mu}, f^{\Lambda}\right]\right] \neq 0$ for any $t \in \mathbb{F}$, as $t e^{-\Lambda}-e^{\mu}$ is a $\mathfrak{b}^{-}$-primitive vector, $\left[t e^{-\Lambda}-e^{\mu}, f^{\Lambda}\right] \neq 0$, and the triple $\left\{f^{\Lambda}, \mathfrak{g}_{0}, t e^{-\Lambda}-e^{\mu}\right\}$ satisfies all the conditions of Theorem 3.17). But $\left[\mathfrak{g}_{1}^{\mu}, f^{\Lambda}\right] \subseteq \mathfrak{t}$ and $\left[\mathfrak{t}, f^{\Lambda}\right]=\mathbb{F} f^{\Lambda}$. This implies that $\left[f^{\Lambda},\left[t_{0} e^{-\Lambda}-e^{\mu}, f^{\Lambda}\right]\right]=0$ for a suitable $t_{0} \in F$. Consequently, $\operatorname{dim} \mathfrak{g}_{1}^{\mu}=1$.

Summarizing, we have that $\mathfrak{g}_{1}$ is an irreducible $\widehat{G}_{0}$-module with maximal weight $-w_{0} \lambda$. As $-w_{0} \lambda \in X_{1}(T)$, Proposition 2.13 shows that $\mathfrak{g}_{1}$ remains irreducible under the action of $(\mathrm{d} \iota)_{e}\left(\operatorname{Lie}\left(\widehat{G}_{0}\right)\right)=\mathfrak{g}_{0}^{(1)}$. Therefore, if $\Lambda^{[k]}$ is minuscule for any $k \in\{1, \ldots, \ell\}$, then we have the desired conclusion that $\mathfrak{g}_{1}$ is an irreducible $\mathfrak{g}_{0}^{(1)}$-module.

It remains to consider the case where the classical algebra $L=\widetilde{\mathfrak{g}} / \mathcal{M}(\widetilde{\mathfrak{g}})$ corresponds to one of the diagrams (I-V) pictured above. In Case (I), we have that $\Phi=\Phi^{[1]} \cup \Phi^{[2]}$ where $\Phi^{[1]}=\mathrm{A}_{m-1}, \Phi^{[2]}=\mathrm{A}_{1}, \Lambda^{[1]}=\varpi_{m-1}^{[1]}$, and $\Lambda^{[2]}=2 \varpi_{1}^{[2]}$. (When $m=1$, we assume that $\Phi^{[1]}=\emptyset$ and $\Lambda^{[1]}=0$.) Since $\lambda \in X_{1}(\widehat{T})$, this yields $\lambda=\varpi_{m-1}^{[1]}+2 \varpi_{1}^{[2]}$, where $\varpi_{m-1}^{[1]}$ and $\varpi_{1}^{[2]}$ are now viewed as fundamental weights in $X(\widehat{T})$. In our situation, $\mathfrak{g}_{-1} \cong L(\lambda) \cong$ $L\left(\varpi_{m-1}^{[1]}\right) \otimes L\left(2 \varpi_{m}^{[2]}\right)$, and $\Phi$ has the following Dynkin diagram: 


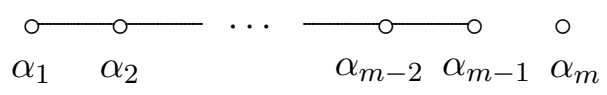

Obviously, $\operatorname{dim} L(\lambda)=3(m+1)$, and $X\left(\mathfrak{g}_{-1}\right)=W \lambda \cup W \varpi_{m-1}^{[1]}$. As $p>3$, it is straightforward to see that all dominant weights of $\operatorname{Hom}\left(\mathfrak{g}_{-1}, \mathfrak{g}_{0}\right)$ belong to $X_{1}(\widehat{T})$. Applying Proposition 2.14 we now obtain that the subspace $\mathfrak{g}_{1}$ is $\widehat{G}_{0}$-stable. The above reasoning then shows that $-\lambda$ is the only minimal weight of the $\widehat{G}_{0}$-module $\mathfrak{g}_{1}$, and $\operatorname{dim} \mathfrak{g}_{1}^{-\lambda}=1$. This, in turn, yields that $\lambda$ is the only maximal weight of the dual $\widehat{G}_{0}$-module $\mathfrak{g}_{1}^{*}$, and $\operatorname{dim}\left(\mathfrak{g}_{1}^{*}\right)^{\lambda}=1$. Let $M$ be the $\widehat{G}_{0}$-submodule of $\mathfrak{g}_{1}^{*}$ generated by a nonzero element $\xi \in\left(\mathfrak{g}_{1}^{*}\right)^{\lambda}$. If $M \neq \mathfrak{g}_{1}^{*}$, then

$$
M^{\perp}=\left\{x \in \mathfrak{g}_{1} \mid \psi(x)=0 \text { for all } \psi \in M\right\}
$$

is a nonzero $\widehat{G}_{0}$-submodule of $\mathfrak{g}_{1}$. As $M^{\perp}$ is $\operatorname{Lie}\left(\widehat{G}_{0}\right)$-stable, it contains a $\mathfrak{b}^{-}$-primitive vector. By our assumption, this forces $M^{\perp} \cap \mathfrak{g}_{1}^{-\Lambda} \neq 0$. As $\mathfrak{g}_{1}^{-\Lambda}=\mathfrak{g}_{1}^{-\lambda}$, we must have that $\mathfrak{g}_{1}^{-\lambda} \subset M^{\perp}$. It follows that $\xi\left(\mathfrak{g}_{1}^{-\lambda}\right)=0$. But $\xi\left(\mathfrak{g}_{1}^{\nu}\right)=0$ for all $\nu \neq-\lambda$ by the definition of $\left(\mathfrak{g}_{1}^{*}\right)^{\lambda}$. Therefore, $\xi=0$ contrary to our assumption.

As a result, $\mathfrak{g}_{1}^{*}$ is generated by a $\mathfrak{b}^{+}$-primitive vector of weight $\lambda$. By Proposition 2.15 , there exists a surjective $\widehat{G}_{0}$-module homomorphism $\psi$ : $V(\lambda) \rightarrow \mathfrak{g}_{1}^{*}$, where $V(\lambda)$ is the Weyl module with maximal weight $\lambda$. In our situation, $V(\lambda) \cong V\left(\varpi_{m-1}^{[1]}\right) \otimes V\left(2 \varpi_{1}^{[2]}\right) \cong L(\lambda)$. This implies that in Case (I), the $\mathfrak{g}_{0}^{(1)}$-module $\mathfrak{g}_{1}$ is irreducible. A similar argument also works in Cases (II) and (III). (In seeing this, one should keep in mind that for groups of type $\mathrm{A}_{m}$, the Weyl modules $V\left(2 \varpi_{1}\right)$ and $V\left(2 \varpi_{m}\right)$ are irreducible provided $p>2$.) In Case $(\mathrm{V})$, we have $\Phi=\Phi^{[1]}=\mathrm{C}_{3}$ and $\Lambda^{[1]}=\varpi_{3}^{[1]}$. It is well-known that in this situation $V\left(\varpi_{3}^{[1]}\right) \cong L\left(\varpi_{3}^{[1]}\right)$ for $p>2$, and $X\left(\mathfrak{g}_{-1}\right)=X\left(L\left(\varpi_{3}^{[1]}\right)\right)=W \varpi_{3}^{[1]} \cup W \varpi_{1}^{[1]}$. Direct verification shows that for $p>3$ all dominant weights of $\operatorname{Hom}\left(\mathfrak{g}_{-1}, \mathfrak{g}_{0}\right)$ are in $X_{1}(\widehat{T})$. Reasoning as above we derive again that $\mathfrak{g}_{1}$ is an irreducible $\mathfrak{g}_{0}^{(1)}$-module.

It remains to consider Case (IV). In this case, $\mathfrak{g}_{0} \cong \mathfrak{g l}_{2}, \Lambda^{[1]}=3 \varpi_{1}$, $\mathfrak{n}^{-}=\mathbb{F} f$, and $\mathfrak{n}^{+}=\mathbb{F} e$. Hence, $\mathfrak{g}_{-1} \cong L(3)$ as $\mathfrak{g}_{0}^{(1)}$-modules (here we adopt the abbreviated notation $L(k)$ for $\left.L\left(k \varpi_{1}\right), 0 \leq k \leq p-1\right)$. Since $\mathfrak{g}_{1}$ is a restricted $\mathfrak{g}_{0}^{(1)}$-module, we have $(\operatorname{ad} f)^{p}\left(\mathfrak{g}_{1}\right)=0$, while the above reasoning yields $\operatorname{dim}\left(\mathfrak{g}_{1} \cap \operatorname{ker}\right.$ ad $\left.f\right)=1$. As a consequence, the $\mathfrak{s l}_{2}$-module $\mathfrak{g}_{1}$ is indecomposable of dimension $\leq p$. Note that $L(3) \cong L(3)^{*}$ and $\mathfrak{g}_{0} \cong$ $L(2) \oplus \mathbb{F}$ as $\mathfrak{g}_{0}^{(1)}$-modules. Therefore,

$$
\mathfrak{g}_{1} \hookrightarrow \operatorname{Hom}\left(\mathfrak{g}_{-1}, \mathfrak{g}_{0}\right) \cong L(3) \oplus(L(2) \otimes L(3)) .
$$


If $p>5$, then the $\mathfrak{s l}_{2}$-module $L(2) \otimes L(3)$ is completely reducible; see [BO] for example. But then $\mathfrak{g}_{1}$ is irreducible, and we are done. So we may assume that $p=5$. Recall that the graded component $\widetilde{\mathfrak{g}}_{1}$ of $\widetilde{\mathfrak{g}}$ is an irreducible $\mathfrak{g}_{0^{-}}$ module generated by a $\mathfrak{b}^{-}$-primitive vector of weight $-\Lambda$. In our case, this says that $\widetilde{\mathfrak{g}}_{1} \cong L(3)$ as $\mathfrak{s l}_{2}$-modules. If $\operatorname{dim} \mathfrak{g}_{1}<5$, then $\mathfrak{g}_{1}=\widetilde{\mathfrak{g}}_{1}$, because $\operatorname{dim} L(3)=4$.

Suppose then that $\operatorname{dim} \mathfrak{g}_{1}=p=5$. As $\widetilde{\mathfrak{g}}_{1} \cong L(3)$, the quotient module $\mathfrak{g}_{1} / \widetilde{\mathfrak{g}}_{1}$ is trivial. As the endomorphism $\left.(\operatorname{ad} h)\right|_{\mathfrak{g}_{1}}$ is semisimple, $\mathfrak{g}_{1}$ contains a nonzero weight vector of weight zero, $u_{0}$ say. By transitivity, we have $\left[u_{0}, \mathfrak{g}_{-1}\right] \neq 0$. Thus, $\mathfrak{g}_{0}$ contains a nonzero weight vector with weight equal to a weight of $\mathfrak{g}_{-1}$. But we know that $\mathfrak{g}_{-1} \cong L(3)$ and $\mathfrak{g}_{0} \cong L(0) \oplus L(2)$. The $h$-eigenvalues of $L(3)$ are $3,1,-1$, and -3 , while the $h$-eigenvalues of $L(0) \oplus L(2)$ are 2,0 , and -2 . Since $p=5$, it must be that either the bracket of a $\mathfrak{b}^{+}$-primitive vector $v_{3}$ of $\mathfrak{g}_{-1}$ with $u_{0}$ is a nonzero scalar multiple of $f$, or the bracket of a $\mathfrak{b}^{-}$-primitive vector $v_{-3}$ of $\mathfrak{g}_{-1}$ with $u_{0}$ is a nonzero scalar multiple of $e$. For definiteness, we will assume that we are in the former situation. The proof in the latter case is similar. We can assume that

$$
\left[v_{3}, u_{0}\right]=f
$$

Since $v_{3}$ is a $\mathfrak{b}^{+}$-primitive vector of $\mathfrak{g}_{-1}$, we have

$$
h=[e, f]=\left[e,\left[v_{3}, u_{0}\right]\right]=\left[v_{3},\left[e, u_{0}\right]\right] .
$$

Set $x_{-3}=\left[e, u_{0}\right]$. Then $x_{-3}$ is an $h$-eigenvector in $\mathfrak{g}_{1}$ with eigenvalue $2 \equiv-3$ $\bmod 5$. It follows that $x_{-3} \in \widetilde{\mathfrak{g}}_{1}$. Since $\widetilde{\mathfrak{g}}_{1} \cong L(3)$ as $\mathfrak{g}_{0}^{(1)}$-modules, it must be that $\left[f, x_{-3}\right]=0$. We thus have

$$
h=\left[v_{3}, x_{-3}\right], \quad\left[e, v_{3}\right]=\left[f, x_{-3}\right]=0 .
$$

But then Theorem 3.7 shows that the subalgebra generated by

$$
\begin{array}{ll}
e_{1}:=e, & f_{1}:=f, \\
e_{2}:=x_{-3}, & f_{2}:=v_{3},
\end{array}
$$

is infinite dimensional. This shows that $\mathfrak{g}_{1}=\widetilde{\mathfrak{g}}_{1}$, completing the proof.

Proposition 4.14. Assume $\mathfrak{g}$ is a graded Lie algebra satisfying assumptions (1)-(4) and (6). Let $e^{\Gamma} \in \mathfrak{g}_{1}$ be a $\mathfrak{b}^{-}$-primitive vector corresponding to a weight $\Gamma \neq-\Lambda$. Then $\Gamma\left(\mathfrak{t}^{[i]}\right) \neq 0$ for all $i=1, \ldots, \ell$.

Proof. Observe that $\mathfrak{g}_{-1}$ is a restricted $\mathfrak{g}_{0}$-module by Remark 4.6. The irreducibility and transitivity of $\mathfrak{g}$ imply that $\mathfrak{Z}\left(\mathfrak{g}_{0}\right)$ acts on $\mathfrak{g}_{-1}$ as scalar operators and this action is faithful. The transitivity of $\mathfrak{g}$ allows us to identify the $\mathfrak{g}_{0}$-module $\mathfrak{g}_{1}$ with a submodule of $\operatorname{Hom}\left(\mathfrak{g}_{-1}, \mathfrak{g}_{0}\right) \cong \mathfrak{g}_{-1}^{*} \otimes \mathfrak{g}_{0}$. As a consequence, $\mathfrak{Z}\left(\mathfrak{g}_{0}\right)$ acts faithfully on $\mathfrak{g}_{1}$.

Now suppose $\Gamma\left(\mathfrak{t}^{[i]}\right)=0$ for some $i$. Without loss of generality we may assume $i=1$. Since $\mathfrak{Z}\left(\mathfrak{g}_{0}\right)$ acts on $\mathfrak{g}_{1}$ as scalar operators, we have $\Gamma(z) \neq 0$ 
for any nonzero $z \in \mathfrak{Z}\left(\mathfrak{g}_{0}\right)$. Then $\mathfrak{Z}\left(\mathfrak{g}_{0}\right) \cap \mathfrak{g}_{0}^{[1]}=0$, showing that $\mathfrak{g}_{0}^{[1]}$ is centerless (in particular, nonabelian). We denote by $\mathfrak{g}_{0,1}$ the derived ideal of $\mathfrak{g}_{0}^{[1]}$. The above remark shows that $\mathfrak{g}_{0,1}$ is classical simple. Let

$$
\mathfrak{g}_{1}=Q_{N} \supset Q_{N-1} \supset \cdots \supset Q_{1} \supset Q_{0}=0
$$

be a composition series for the $\mathfrak{g}_{0}$-module $\mathfrak{g}_{1}$, and let $\Gamma_{k}$ denote the weight of a $\mathfrak{b}^{-}$-primitive vector of $Q_{k} / Q_{k-1}$. Since $\mathfrak{g}_{1}$ is a submodule of the restricted $\mathfrak{g}_{0}^{(1)}$-module $\mathfrak{g}_{-1}^{*} \otimes \mathfrak{g}_{0}$, all the composition factors $Q_{k} / Q_{k-1}$ are irreducible, restricted $\mathfrak{g}_{0}^{(1)}$-modules. It follows that $Q_{k} / Q_{k-1}$ is a completely reducible $\mathfrak{g}_{0,1^{-}}$ module generated by $\mathfrak{b}^{-}$-primitive vectors of weight $\Gamma_{k} \mid \mathfrak{t} \cap \mathfrak{g}_{0,1}$. If $\Gamma_{k}\left(\mathfrak{t}^{[1]}\right)=0$ for all $k$, then $Q_{k} / Q_{k-1}$ is a trivial $\mathfrak{g}_{0,1}$-module for all $k$. However, if $Q_{k-1}$ and $Q_{k} / Q_{k-1}$ are trivial $\mathfrak{g}_{0,1}$-modules, then so is $Q_{k}$. Indeed, for any $v \in Q_{k}$, we have $x . v \in Q_{k-1}$ for all $x \in \mathfrak{g}_{0,1}$. But then $[x, y] v=x .(y . v)-y \cdot(x . v)=0$ for all $x, y \in \mathfrak{g}_{0,1}$ so that $Q_{k}$ is a trivial $\mathfrak{g}_{0,1}$-module too, because $\mathfrak{g}_{0,1}$ is a simple Lie algebra. This would lead to $\mathfrak{g}_{1}$ being a trivial $\mathfrak{g}_{0,1}$-module, which cannot happen since $\mathfrak{g}_{1}$ is assumed to be a faithful $\mathfrak{g}_{0}$-module. Consequently, $\Gamma_{k}\left(\mathfrak{t}^{[1]}\right) \neq 0$ for some $k$. We may suppose that $k$ is chosen so $\Gamma_{l}\left(\mathfrak{t}^{[1]}\right)=0$ for all $l<k$ but $\Gamma_{k}\left(\mathfrak{t}^{[1]}\right) \neq 0$.

Let $e^{\Gamma_{k}}$ be a weight vector of $Q_{k}$ whose image in $Q_{k} / Q_{k-1}$ is a nonzero $\mathfrak{b}^{-}$-primitive vector. We claim that $e^{\Gamma_{k}}$ itself is a $\mathfrak{b}^{-}$-primitive vector. First note that $Q_{k-1}$ is a trivial $\mathfrak{g}_{0}^{[1]}$-module, so that $\mu\left(\mathfrak{t}^{[1]}\right)=0$ for any weight $\mu$ of the $\mathfrak{g}_{0}$-module $Q_{k-1}$ (an immediate consequence of our choice of $k$ ). This implies that $\left[e_{\theta}, e^{\Gamma_{k}}\right]=0$ for any $\theta \in\left(\Phi^{[i]}\right)^{-}$with $i>1$. Suppose that $u:=\left[e_{-\alpha_{j}}, e^{\Gamma_{k}}\right] \neq 0$ for some simple root $\alpha_{j}$ of $\Phi^{[1]}$. As $u \in Q_{k-1}$ is a weight vector corresponding to the weight $\Gamma_{k}-\alpha_{j}$, we have $\left(\Gamma_{k}-\alpha_{j}\right)\left(\mathfrak{t}^{[1]}\right)=0$. Further, $\left[e_{\theta}, u\right]=\left[e_{-\alpha_{j}},\left[e_{\theta}, e^{\Gamma_{k}}\right]\right]=0$ for any $\theta \in\left(\Phi^{[i]}\right)^{-}$with $i>1$. Thus, $\left[\left(\mathfrak{n}^{[i]}\right)^{-}, u\right]=0$ for all $i=1, \ldots, \ell$, which is to say that $u$ is a $\mathfrak{b}^{-}$-primitive vector. Then it follows that $\left[f^{\Lambda}, u\right] \neq 0$ by the transitivity of $\mathfrak{g}$. As $\Lambda\left(\mathfrak{t}^{[1]}\right) \neq$ 0 by transitivity (compare Remark 4.5), we have $\left[f^{\Lambda}, u\right]=\zeta e_{\beta}$ for some $\zeta \in \mathbb{F}^{\times}$and $\beta \in \Phi^{[1]}$. Since $u \in \mathfrak{g}_{0}^{[1]}$, which is centerless, and $\left[e_{\alpha}, u\right]=0$ for any $\alpha \in\left(\Phi^{[1]}\right)^{+}$, we see that $\beta$ is the highest root of $\Phi^{[1]}$. Observe that $\Lambda+\Gamma_{k}=\beta+\alpha_{j}$ as functions on $\mathfrak{t}$. Since $p>3$, the function $\beta+\alpha_{j} \in \mathfrak{t}^{*}$ is not a root of $\mathfrak{g}_{0}^{[1]}$. Therefore, $\left[f^{\Lambda}, e^{\Gamma_{k}}\right]=0$.

Now if $\left[e_{-\alpha_{l}}, e^{\Gamma_{k}}\right]$ were nonzero for some $l \neq j$, the above argument would yield $\Lambda+\Gamma_{k}=\beta+\alpha_{l}$, which would force $\alpha_{l}=\alpha_{j}$. Hence, $\left[e_{-\alpha_{l}}, e^{\Gamma_{k}}\right]=0$ for all $l \neq j$. Using that property and the fact that $\left[e_{-\alpha_{l}},\left[e_{-\alpha_{j}}, e^{\Gamma_{k}}\right]\right]=0$ for all $l$, it is easy to verify that $\left[e_{-\nu}, e^{\Gamma_{k}}\right]=0$ for all $\nu \in \Phi^{+}, \nu \neq \alpha_{j}$.

Suppose $\beta \neq \alpha_{j}$. Using the relations obtained, we get

$$
\begin{aligned}
{\left[\left[\left[\left[f^{\Lambda}, e_{-\alpha_{j}}\right], e_{-\beta}\right], e_{-\beta}\right], e^{\Gamma_{k}}\right] } & =\left[\left[\left[f^{\Lambda}, u\right], e_{-\beta}\right], e_{-\beta}\right] \\
& =\zeta\left[\left[e_{\beta}, e_{-\beta}\right], e_{-\beta}\right]=-2 \zeta e_{-\beta} \neq 0 .
\end{aligned}
$$


This yields that $\left[\left[\left[f^{\Lambda}, e_{-\alpha_{j}}\right], e_{-\beta}\right], e_{-\beta}\right] \neq 0$. Since $\mathfrak{g}_{-1}$ is an irreducible $\mathfrak{g}_{0}$-module and $f^{\Lambda}$ is a $\mathfrak{b}^{+}$-primitive vector of $\mathfrak{g}_{-1}$, the subspace $P:=$ $\mathfrak{U}\left(\left(\mathfrak{n}^{[1]}\right)^{-}\right) \cdot f^{\Lambda} \subseteq \mathfrak{g}_{-1}$ generated by $f^{\Lambda}$ is an irreducible $\mathfrak{g}_{0}^{[1]}$-module with a $\left(\mathfrak{b}^{[1]}\right)^{+}$-primitive vector of weight $\Lambda^{[1]}=\left.\Lambda\right|_{\mathfrak{t}^{[1]}}$. It follows from $\left(\Gamma_{k}-\right.$ $\left.\alpha_{j}\right)\left(\mathfrak{t}^{[1]}\right)=0$ that $\Lambda^{[1]}=\beta$. Since the Lie algebra $\mathfrak{g}_{0,1}$ is simple, the $\mathfrak{g}_{0}^{[1]}$ modules $\mathfrak{g}_{0,1}$ and $P$ are isomorphic. Moreover, because $\beta$ is a long root of $\Phi^{[1]}$, we must have

$$
\left[\left[P, e_{-\beta}\right], e_{-\beta}\right] \subseteq P^{-\beta} \quad(\text { the }-\beta \text { weight space of } P) .
$$

However, this contradicts the fact that $0 \neq\left[\left[\left[f^{\Lambda}, e_{-\alpha_{j}}\right], e_{-\beta}\right], e_{-\beta}\right] \in P^{-\beta-\alpha_{j}}$. Consequently, the case $\beta \neq \alpha_{j}$ cannot occur.

So let us suppose that $\beta=\alpha_{j}$. Then

$$
\begin{aligned}
{\left[\left[\left[\left[f^{\Lambda}, e_{-\alpha_{j}}\right], e_{-\alpha_{j}}\right], e_{-\alpha_{j}}\right], e^{\Gamma_{k}}\right] } & =3\left[\left[\left[f^{\Lambda}, u\right], e_{-\alpha_{j}}\right], e_{-\alpha_{j}}\right] \\
& =3 \zeta\left[\left[e_{\alpha_{j}}, e_{-\alpha_{j}}\right], e_{-\alpha_{j}}\right] \\
& =-6 \zeta e_{-\alpha_{j}} \neq 0
\end{aligned}
$$

whence $0 \neq\left[\left[\left[f^{\Lambda}, e_{-\alpha_{j}}\right], e_{-\alpha_{j}}\right], e_{-\alpha_{j}}\right] \in P^{-2 \beta}$. But since $P$ is isomorphic to $\mathfrak{g}_{0,1}$ as $\mathfrak{g}_{0}^{[1]}$-modules, it is impossible for $-2 \beta$ to be a weight of $P$. This contradiction establishes the claim that $e^{\Gamma_{k}}$ is a $\mathfrak{b}^{-}$-primitive vector. As a consequence, $\left[f^{\Lambda}, e^{\Gamma_{k}}\right] \neq 0$ by the transitivity of $\mathfrak{g}$.

Suppose $\Gamma_{k}=-\Lambda$. Let $\widetilde{\mathfrak{g}}_{1}$ denote the $\mathfrak{g}_{0}$-module generated by $e^{-\Lambda}$. If $\widetilde{\mathfrak{g}}_{1}$ is irreducible then $\Gamma\left(\mathfrak{t}^{[1]}\right) \neq 0$ by Theorem 4.7 (i), contradicting our initial assumption. Consequently, $\widetilde{\mathfrak{g}}_{1} \cap Q_{k-1} \neq 0$. We may thus assume without loss of generality that $e^{\Gamma_{1}} \in \widetilde{\mathfrak{g}}_{1}$. For all positive roots $\alpha \in \Phi^{[1]}$, choose $e_{\alpha} \in \mathfrak{g}_{0}^{\alpha}, e_{-\alpha} \in \mathfrak{g}_{0}^{-\alpha}$, and $h_{\alpha} \in \mathfrak{t} \operatorname{such}$ that $\left(e_{\alpha}, h_{\alpha}, e_{-\alpha}\right)$ form an $\mathfrak{s l}_{2}$-triple in $\mathfrak{g}_{0,1}$. Recall that $\left[e_{-\alpha}, e^{\Gamma_{1}}\right]=0$ for all $\alpha \in \Phi^{[1]}$. Since $\left[f^{\Lambda}, e^{\Gamma_{1}}\right] \neq 0$ by the transitivity of $\mathfrak{g}$ and the definition of $k$ which implies that $e^{\Gamma_{1}}$ has zero $\mathfrak{g}_{0,1}$-weight, the linear map ad $e^{\Gamma_{1}}$ induces an isomorphism between the $\mathfrak{g}_{0}^{[1]}$ modules $P$ and $\mathfrak{g}_{0,1}$. Note that $\left[f^{\Lambda}, e^{\Gamma_{1}}\right]=e_{\beta}$, after rescaling $e_{\beta}$ possibly. Since $\mathfrak{g}$ is transitive, Theorem 3.17 yields $\Lambda\left(h_{\Lambda}\right) \neq 0$. Rescaling $e^{-\Lambda}$ if necessary we may assume that $\Lambda\left(h_{\Lambda}\right)=2$.

Suppose $\Phi^{[1]} \cong \mathrm{A}_{1}$. Then the endomorphism

$$
C:=E_{\beta} E_{-\beta}+E_{-\beta} E_{\beta}+\frac{1}{2} H_{\beta}^{2}
$$

of $\mathfrak{g}$ commutes with ad $\mathfrak{g}_{0}$ and hence acts on $\widetilde{\mathfrak{g}}_{1}$ as $\mu$ id where

$$
\mu=\frac{1}{2} \Lambda\left(h_{\beta}\right)^{2}+\Lambda\left(h_{\beta}\right)=\frac{1}{2} \beta\left(h_{\beta}\right)^{2}+\beta\left(h_{\beta}\right)=4 .
$$

But then $4 e^{\Gamma_{1}}=C\left(e^{\Gamma_{1}}\right)=0$, a contradiction. This shows that the rank of the root system $\Phi^{[1]}$ is $\geq 2$. 
For $\alpha \in\left(\Phi^{[1]}\right)^{+}$, set $e_{1}=e_{-\alpha}, e_{2}=f_{\Lambda}, f_{1}=e_{\alpha}, f_{2}=e_{-\Lambda}$. Clearly, $\left[e_{i}, f_{j}\right]=0$ for $i \neq j$ and $\left[h_{i}, e_{i}\right]=2 e_{i}$, where $i, j \in\{1,2\}$. Let $\mathfrak{k}_{\alpha}$ denote the subalgebra of $\mathfrak{g}$ generated by $e_{i}, f_{i}, i=1,2$. Set $h_{1}=-h_{\alpha}$ and $h_{2}=h_{\Lambda}$. Since $h_{1} \in \mathfrak{g}_{0,1}$ we have $\Lambda\left(h_{1}\right)=-\beta\left(h_{\alpha}\right)$. The matrix $A_{\alpha}$ of the Lie algebra $\mathfrak{k}_{\alpha}$ has the form

$$
A_{\alpha}=\left(\begin{array}{cc}
2 & \Lambda\left(h_{1}\right) \\
-\alpha\left(h_{\Lambda}\right) & 2
\end{array}\right) .
$$

Since $\alpha\left(h_{\beta}\right)=0$ if and only if $\beta\left(h_{\alpha}\right)=-\Lambda\left(h_{1}\right)=0$, we now apply Theorem 3.8 to deduce that

$$
\alpha\left(h_{\beta}\right)=0 \Longleftrightarrow \alpha\left(h_{\Lambda}\right)=0 .
$$

Now let $\alpha_{s}$ be any simple root in $\Phi^{[1]}$ with $\beta-\alpha_{s} \in\left(\Phi^{[1]}\right)^{+}$(such a root exists because $\Phi^{[1]}$ is not of type $A_{1}$ ). Then we have

$$
\left[e_{-\gamma},\left[e^{-\Lambda}, e^{\Gamma_{1}}\right]\right]=0=\left[e_{\gamma},\left[f^{\Lambda},\left[f^{\Lambda}, e_{-\alpha_{s}}\right]\right]\right]
$$

for all $\gamma \in \Phi^{+}$. Since $\Gamma_{1}-\beta=-\Lambda$ we also have

$$
\begin{gathered}
{\left[\left[f^{\Lambda},\left[f^{\Lambda}, e_{-\alpha_{s}}\right]\right],\left[e^{-\Lambda}, e^{\Gamma_{1}}\right]\right]=\left[\left[f^{\Lambda},\left[e^{-\Lambda}, e^{\Gamma_{1}}\right]\right],\left[f^{\Lambda}, e_{-\alpha_{s}}\right]\right]} \\
+\left[f^{\Lambda},\left[\left[f^{\Lambda},\left[e^{-\Lambda}, e^{\Gamma_{1}}\right]\right], e_{-\alpha_{s}}\right]\right] \\
=\Gamma_{1}\left(h_{\Lambda}\right)\left[e^{\Gamma_{1}},\left[f^{\Lambda}, e_{-\alpha_{s}}\right]\right]+\left[\left[e^{-\Lambda}, e_{\beta}\right],\left[f^{\Lambda}, e_{-\alpha_{s}}\right]\right] \\
+\Gamma_{1}\left(h_{\Lambda}\right)\left[f^{\Lambda},\left[e^{\Gamma_{1}}, e_{-\alpha_{s}}\right]\right]+\left[f^{\Lambda},\left[\left[e^{-\Lambda}, e_{\beta}\right], e_{-\alpha_{s}}\right]\right] \\
=-\Gamma_{1}\left(h_{\Lambda}\right)\left[e_{\beta}, e_{-\alpha_{s}}\right]-\left[e_{\beta},\left[e^{-\Lambda},\left[f^{\Lambda}, e_{-\alpha_{s}}\right]\right]\right]+\left[f^{\Lambda},\left[e^{-\Lambda},\left[e_{\beta}, e_{-\alpha_{s}}\right]\right]\right] \\
=-\Gamma_{1}\left(h_{\Lambda}\right)\left[e_{\beta}, e_{-\alpha_{s}}\right]+\left[e_{\beta},\left[h_{\Lambda}, e_{-\alpha_{s}}\right]\right]+\left[h_{\Lambda},\left[e_{\beta}, e_{-\alpha_{s}}\right]\right] \\
=-\left(\Gamma_{1}+\alpha_{s}-\beta+\alpha_{s}\right)\left(h_{\Lambda}\right)\left[e_{\beta}, e_{-\alpha_{s}}\right]=\left(\Lambda-2 \alpha_{s}\right)\left(h_{\Lambda}\right)\left[e_{\beta}, e_{-\alpha_{s}}\right] .
\end{gathered}
$$

Next observe that $2 \Lambda-\alpha_{s}$ and $-\Lambda+\Gamma_{1}=-2 \Lambda+\beta$ do not vanish on $\mathfrak{t}^{[i]}$ for $i \geq 2$ because $\alpha_{s}, \beta \in \Phi^{[1]}$ (see Remark 4.5). Also, $(-2 \Lambda+\beta)\left(h_{\beta}\right)=$ $-\beta\left(h_{\beta}\right) \neq 0$ and

$$
\left(2 \Lambda-\alpha_{s}\right)\left(h_{\beta}\right)=2 \beta\left(h_{\beta}\right)-\alpha_{s}\left(h_{\beta}\right)=4-2 \frac{\left(\alpha_{s}, \beta\right)}{(\beta, \beta)} \neq 0
$$

as $\left(\alpha_{s}, \beta\right)=\left(\beta, \alpha_{s}\right)>0$. Applying Theorem 4.4 to the graded Lie algebra $\bigoplus_{i \in \mathbb{Z}} \mathfrak{g}_{2 i}$, we are now able to deduce that $\left(\Lambda-2 \alpha_{s}\right)\left(h_{\Lambda}\right)=0$ since neither $2 \Lambda-\alpha_{s}$ nor $-2 \Lambda+\beta$ is the highest or lowest weight of a standard representation for either $\mathrm{A}_{r}$ or $\mathrm{C}_{r}$. It follows that

$$
\alpha_{s}\left(h_{\Lambda}\right)=1 \quad \text { whenever } \alpha_{s} \in \Delta \text { and } \beta-\alpha_{s} \in \Phi .
$$

Let $\Delta_{\beta}$ denote the set of all simple roots $\alpha \in\left(\Phi^{[1]}\right)^{+}$with $(\beta, \alpha)=0$. If $\Phi^{[1]}$ is not of type $\mathrm{A}_{r}, r \geq 2$, then there is a unique $\alpha_{s} \in \Delta$ such $\beta-\alpha_{s} \in \Phi$ and $\beta=2 \alpha_{s}+\sum_{\alpha \in \Delta_{\beta}} r_{\alpha} \alpha$ for some $r_{\alpha} \in \mathbb{Z}$. If $\Phi^{[1]}$ is of type $\mathrm{A}_{r}, r \geq 2$, 
then $\beta-\alpha_{s} \in \Phi$ for $s \in\{1, r\}$ and $\beta=\alpha_{1}+\alpha_{r}+\sum_{\alpha \in \Delta_{\beta}} \alpha$. In conjunction with (4.16) and (4.17) this yields $\beta\left(h_{\Lambda}\right)=2$. As a result, if $A_{\beta}$ denotes the matrix in (4.15) with $\beta$ in place of $\alpha$, we have

$$
A_{\beta}=\left(\begin{array}{cc}
2 & -2 \\
-2 & 2
\end{array}\right)
$$

see (4.15). But then $h_{1}+h_{2}$ lies in the center of the subalgebra $\mathfrak{k}_{\beta}$ generated by $e_{1}=e_{-\beta}, e_{2}=f_{\Lambda}, f_{1}=e_{\beta}, f_{2}=e_{-\Lambda}$, and the graded algebra $\mathfrak{k}_{\beta} / \mathbb{F}\left(h_{1}+\right.$ $h_{2}$ ) satisfies the conditions of Proposition 3.6. Since $\mathfrak{g}$ is finite-dimensional, this is impossible in view of Theorem 3.7. Thus, $\Gamma_{k} \neq-\Lambda$.

If $\Gamma_{k}\left(\mathfrak{t}^{[i]}\right) \neq 0$ for all $i \in\{1, \ldots, \ell\}$, then Theorem 4.4 would imply that $\Phi=\Phi^{[1]}$ and either $\Phi=\mathrm{A}_{m}, \Lambda \in\left\{\varpi_{1}, \varpi_{m}\right\}$, or $\Phi=\mathrm{C}_{m}, \Lambda=\varpi_{1}$. Since $\left.\operatorname{ad} e^{\Gamma}\right|_{\mathfrak{g}-1} \in \operatorname{Hom}\left(\mathfrak{g}_{-1}, \mathfrak{g}_{0}\right)^{\mathfrak{t}^{[1]}+\left(\mathfrak{n}^{[1]}\right)^{-}} \cong\left(\mathfrak{g}_{-1}^{*} \otimes \mathfrak{g}_{0}\right)^{\mathfrak{t}^{[1]}+\left(\mathfrak{n}^{[1]}\right)^{-}}$, this would say that $\mathfrak{g}_{-1}^{*} \otimes \mathfrak{g}_{0}$ has a $\mathfrak{b}^{-}$-primitive vector of weight zero, which implies that $\mathfrak{g}_{0}$ has a minuscule weight (see (2.9)). As we have noted in the proof of Lemma 4.7(i), this is impossible. Consequently, we must have $\ell>1$ and $\Gamma_{k}\left(\mathfrak{t}^{[i]}\right)=0$ for some $i \in\{2, \ldots, \ell\}$. We may assume that $i=2$. Since by transitivity, $\Lambda\left(\mathfrak{t}^{[l]}\right) \neq 0$ for all $l \in\{1, \ldots, \ell\}$ (see Remark 4.5), we have $\left[f^{\Lambda}, e^{\Gamma}\right]=\xi e_{\mu}$ and $\left[f^{\Lambda}, e^{\Gamma_{k}}\right]=\xi^{\prime} e_{\nu}$ for some $\mu \in \Phi^{[1]}, \nu \in \Phi^{[2]}$, and scalars $\xi, \xi^{\prime}$ which are nonzero (again by transitivity). If $\mu \in \Phi^{-}$or $\nu \in \Phi^{-}$, then $\ell=1$ by Theorem 4.4. Therefore, it must be that $\mu, \nu \in \Phi^{+}$. By adjusting $e^{\Gamma}$ and $e^{\Gamma_{k}}$ by scalars if necessary, we may assume that $\xi=1=\xi^{\prime}$. Set

$$
\begin{array}{cc}
e_{1}=\left[f^{\Lambda}, e_{-\mu}\right] & e_{2}=\left[f^{\Lambda}, e_{-\nu}\right] \\
f_{1}=e^{\Gamma} & f_{2}=e^{\Gamma_{k}} \\
h_{1}=h_{\mu} & h_{2}=h_{\nu} .
\end{array}
$$

It is easy to check that $\left[e_{i}, f_{j}\right]=\delta_{i, j} h_{i}$ for $i, j \in\{1,2\}$. As $\Gamma\left(\mathfrak{t}^{[1]}\right)=\Gamma_{k}\left(\mathfrak{t}^{[2]}\right)=$ 0 , we have that $\left.\Lambda\right|_{\mathfrak{t}^{[1]}}=\left.\mu\right|_{\mathfrak{t}^{[1]}}$ and $\left.\Lambda\right|_{\mathfrak{t}^{[2]}}=\left.\nu\right|_{\mathfrak{t}^{[2]}}$. This implies that $\left[h_{i}, e_{i}\right]=$ $\left[h_{i}, f_{i}\right]=0($ for $i=1,2),\left[h_{1}, e_{2}\right]=2 e_{2},\left[h_{2}, e_{1}\right]=2 e_{1},\left[h_{1}, f_{2}\right]=-2 f_{2}$ and $\left[h_{2}, f_{1}\right]=-2 f_{1}$. But then the Lie algebra generated by the $e_{i}, f_{i}, h_{i}, i=1,2$ is infinite-dimensional by Theorem 3.7. We have reached a contradiction, so it must be that $\Gamma\left(\mathfrak{t}^{[i]}\right) \neq 0$ for all $i$.

\subsection{Determination of the local Lie algebra}

Here we determine the Lie algebra generated by the local part $\mathfrak{g}_{-1} \oplus$ $\mathfrak{g}_{0} \oplus \mathfrak{g}_{1}$ and show that it must be one of the restricted Lie algebras of Cartan type. If $\mathfrak{g}_{-1}$ has a $\mathfrak{b}^{+}$-primitive vector of weight $\Lambda$ and $\mathfrak{g}_{1}$ has a $\mathfrak{b}^{-}$-primitive vector of weight $\Gamma$, then a classical Lie algebra is generated if $\Gamma=-\Lambda$. We analyze which classical Lie algebras can be obtained by adding $-\Lambda$ to the root system of $\mathfrak{g}_{0}$. Of course, since we are in the noncontragredient case, there must be more to $\mathfrak{g}$ than just that larger classical Lie algebra, and we must determine that part as well. 
Given a rational module $M$ over a reductive group $G$ we denote by $X_{+}(M)$ the set of dominant weights of $M$ relative to a maximal torus of $G$.

THEOREM 4.18. Let $\mathfrak{g}$ be a graded Lie algebra satisfying conditions (1)(5). Suppose $\mathfrak{g}$ is generated by its local part $\mathfrak{g}_{-1} \oplus \mathfrak{g}_{0} \oplus \mathfrak{g}_{1}$, and assume there exist $a \mathfrak{b}^{+}$-primitive vector $f^{\Lambda} \in \mathfrak{g}_{-1}$ of weight $\Lambda$ and $a \mathfrak{b}^{-}$-primitive vector $e^{\Gamma} \in \mathfrak{g}_{1}$ of weight $\Gamma$ such that $\left[f^{\Lambda}, e^{\Gamma}\right]=e_{-\alpha}$ where $\alpha \in \Phi^{+}$. Then the graded Lie algebra $\mathfrak{g}$ is isomorphic to a restricted Lie algebra of Cartan type with its natural grading. More precisely, $\mathfrak{g}$ is either $W(m ; \underline{1}), m \geq 2$, or $S(m ; \underline{1})^{(1)}, m \geq 3$, or $S(m ; \underline{1})^{(1)} \oplus \mathbb{F} \mathfrak{D}_{1}, m \geq 3$, or $H(2 m, \underline{1})^{(2)}, m \geq 1$, or $H(2 m ; \underline{1})^{(2)} \oplus \mathbb{F} \mathfrak{D}_{1}, m \geq 3$, or $K(2 m+1, \underline{1})^{(1)}, m \geq 1$.

Proof. Combining Proposition 4.14 with Theorem 4.4 we see that the root system $\Phi$ of $\mathfrak{g}_{0}^{(1)}$ is of type $\mathrm{A}_{m}$ or $\mathrm{C}_{m} ; \alpha$ is the highest root of $\Phi$; and $\mathfrak{g}_{-1}$ is a standard $\mathfrak{g}_{0}^{(1)}$-module. Hence the weight $\Lambda$ of $\mathfrak{g}_{-1}$ is a minuscule weight. Replacing the base $\Delta=\left\{\alpha_{1}, \ldots, \alpha_{m}\right\}$ of $\Phi$ by $\left\{\alpha_{1}^{\prime}=\alpha_{m}, \alpha_{2}^{\prime}=\right.$ $\left.\alpha_{m-1}, \ldots, \alpha_{m}^{\prime}=\alpha_{1}\right\}$ if necessary, we may assume that $\Lambda=\varpi_{m}$ if $\Phi$ is of type $\mathrm{A}_{m}$. Thus, we may assume that either $\mathfrak{g}_{-1}=V$ and $\mathfrak{s p}(V) \subseteq \mathfrak{g}_{0} \subseteq$ $\mathfrak{c s p}(V)$ or $\mathfrak{g}_{-1}=V^{*}$ and $\mathfrak{s l}(V) \subseteq \mathfrak{g}_{0} \subseteq \mathfrak{g l}(V)$, where $V$ is the standard $\mathfrak{g}_{0}^{(1)}$-module. There is a connected, reductive subgroup $\widetilde{G}_{0}$ in $\operatorname{GL}(V)$ with $\operatorname{Lie}\left(\widetilde{G}_{0}\right)=\mathfrak{g}_{0}$ such that the adjoint action of $\mathfrak{g}_{0}$ on $\mathfrak{g}_{-1} \oplus \mathfrak{g}_{0}$ is induced by the differential of the natural action of $\widetilde{G}_{0}$. Moreover, $\widetilde{G}_{0}$ contains a maximal torus $\widetilde{T}$ and maximal unipotent subgroups $N^{ \pm}$such that $\operatorname{Lie}(T)=\mathfrak{t} \cap \mathfrak{g}_{0}^{(1)}$ and $\operatorname{Lie}\left(N^{ \pm}\right)=\mathfrak{n}^{ \pm}$. Let $G_{0}$ denote the derived subgroup of $\widetilde{G}_{0}$ and put $T=\widetilde{T} \cap G_{0}$. Then $T$ is a maximal torus of $G_{0}$ with $\operatorname{Lie}(T)=\mathfrak{t} \cap \mathfrak{g}_{0}^{(1)}$ and $\operatorname{Lie}\left(G_{0}\right)=\mathfrak{g}_{0}^{(1)}$.

(a) The transitivity of $\mathfrak{g}$ allows us to identify $\mathfrak{g}_{1}$ with a $\mathfrak{g}_{0}$-submodule of $\operatorname{Hom}\left(\mathfrak{g}_{-1}, \mathfrak{g}_{0}\right)$. The above discussion shows that the adjoint action of $\mathfrak{g}_{0}$ on $\mathfrak{g}_{1}$ is obtained by restricting to $\mathfrak{g}_{1}$ the differential of the natural rational action of $G_{0}$ on $\operatorname{Hom}\left(\mathfrak{g}_{-1}, \mathfrak{g}_{0}\right) \cong \mathfrak{g}_{-1}^{*} \otimes \mathfrak{g}_{0}$.

Suppose $\mathfrak{g}_{0}^{(1)}$ is of type $\mathrm{A}_{m}, m \geq 2$. Then $\operatorname{Hom}\left(\mathfrak{g}_{-1}, \mathfrak{g}_{0}\right) \hookrightarrow V \otimes \mathfrak{g l}(V)$ is isomorphic to a $G_{0}$-submodule of the rational $G_{0}$-module $V \otimes V \otimes V^{*}$. Since the set of $\widetilde{T}$-weights of $V$ equals $\left\{\varepsilon_{1}, \ldots, \varepsilon_{m+1}\right\}$ and $p>2$, we have

$$
\begin{aligned}
X_{+}\left(\operatorname{Hom}\left(\mathfrak{g}_{-1}, \mathfrak{g}_{0}\right)\right) & \subseteq X_{+}\left(V \otimes V \otimes V^{*}\right) \\
& =\left\{2 \varpi_{1}+\varpi_{m}, \varpi_{2}+\varpi_{m}, \varpi_{1}\right\} \\
& \subseteq X_{1}(T),
\end{aligned}
$$

where $\varpi_{2}+\varpi_{m}$ is omitted if $m=2$. Hence any $\mathfrak{g}_{0}$-submodule of $\mathfrak{g}_{1}$ is $G_{0}$-stable by Proposition 2.15 .

Suppose $\mathfrak{g}_{0}^{(1)}$ is of type $\mathrm{C}_{m}, m \geq 1$. Then $V \cong V^{*}$ and $\mathfrak{g}_{0}^{(1)}=\mathfrak{s p}(V) \cong$ $S^{2}(V)$ as $G_{0}$-modules. Hence $\operatorname{Hom}\left(\mathfrak{g}_{-1}, \mathfrak{g}_{0}\right) \hookrightarrow V \otimes(\mathfrak{s p}(V) \oplus \mathbb{F})$ is isomorphic 
to a $G_{0}$-submodule of $V \oplus\left(S^{2}(V) \otimes V\right)$. Since $X(V)=\left\{ \pm \varepsilon_{1}, \ldots, \pm \varepsilon_{m}\right\}$ and $p>3$, we have

$$
\begin{aligned}
X_{+}\left(\operatorname{Hom}\left(\mathfrak{g}_{-1}, \mathfrak{g}_{0}\right)\right) & \subseteq X_{+}(V) \cup X_{+}\left(S^{2}(V) \otimes V\right) \\
& =\left\{3 \varpi_{1}, \varpi_{1}+\varpi_{2}, \varpi_{3}, \varpi_{1}\right\} \\
& \subseteq X_{1}(T),
\end{aligned}
$$

where $\varpi_{1}+\varpi_{2}$ is omitted if $m=1$ and $\varpi_{3}$ is omitted if $m=1,2$. Again Proposition 2.15 applies to show that any $\mathfrak{g}_{0}$-submodule of $\mathfrak{g}_{1}$ is an $G_{0}$-stable.

(b) Let $\mathfrak{g}_{1}^{\prime}$ be the $\mathfrak{g}_{0}$-submodule of $\mathfrak{g}_{1}$ generated by $e^{\Gamma}$. Let $\mathfrak{U}\left(\mathfrak{n}^{-}\right)$denote the universal enveloping algebra of the Lie algebra $\mathfrak{n}^{-}$. As $\mathfrak{g}_{-1}$ is irreducible, $\mathfrak{g}_{-1}=\mathfrak{U}\left(\mathfrak{n}^{-}\right) f^{\Lambda}$. Therefore, $\left[e^{\Gamma}, \mathfrak{g}_{-1}\right] \subseteq \mathfrak{U}\left(\mathfrak{n}^{-}\right)\left[e^{\Gamma}, f^{\Lambda}\right]=\mathfrak{U}\left(\mathfrak{n}^{-}\right) e_{-\alpha}=\mathbb{F} e_{-\alpha}$. Moreover, $\left[\mathfrak{n}^{-}, \mathfrak{g}_{-1}\right] \subseteq \operatorname{ker}$ ad $e^{\Gamma}$ as $\left[\mathfrak{n}^{-}, e_{-\alpha}\right]=0$. Since $\mathfrak{g}_{-1}$ is standard, we have $\mathfrak{g}_{-1}=\mathbb{F} f^{\Lambda} \oplus\left[\mathfrak{n}^{-}, \mathfrak{g}_{-1}\right]$ and $\left[e_{-\alpha}, \mathfrak{g}_{-1}\right]=\mathbb{F} e^{\Omega}$ where $e^{\Omega}$ is a $\mathfrak{b}^{-}$-primitive vector of $\mathfrak{g}_{-1}$. But then

$$
\begin{aligned}
{\left[e^{\Gamma},\left[\mathfrak{g}_{-1}, \mathfrak{g}_{-1}\right]\right] } & =\left[e^{\Gamma},\left[\left(\mathbb{F} f^{\Lambda}+\left[\mathfrak{n}^{-}, \mathfrak{g}_{-1}\right]\right),\left(\mathbb{F} f^{\Lambda}+\left[\mathfrak{n}^{-}, \mathfrak{g}_{-1}\right]\right)\right]\right] \\
& =\left[e^{\Gamma},\left[\mathfrak{g}_{-1},\left[\mathfrak{n}^{-}, \mathfrak{g}_{-1}\right]\right]=\left[\left[e^{\Gamma}, \mathfrak{g}_{-1}\right],\left[\mathfrak{n}^{-}, \mathfrak{g}_{-1}\right]\right]\right. \\
& =\left[e_{-\alpha},\left[\mathfrak{n}^{-}, \mathfrak{g}_{-1}\right]\right]=\left[\mathfrak{n}^{-}, e^{\Omega}\right]=0 .
\end{aligned}
$$

This implies that $\left[\mathfrak{g}_{1}^{\prime}, \mathfrak{g}_{-2}\right]=0$.

(c) Let $e^{\Xi}$ be a $\mathfrak{b}^{-}$-primitive vector from $\mathfrak{g}_{1}$ of weight $\Xi$. If $\Xi \neq-\Lambda$, then $\left[f^{\Lambda}, e^{\Xi}\right] \in \mathbb{F}^{\times} e_{-\delta}$ for some $\delta \in \Phi$ by transitivity (1.2). Since no minuscule weight is a root, $\Xi\left(\mathfrak{t} \cap \mathfrak{g}_{0}^{(1)}\right) \neq 0$. If $\delta \in \Phi^{-}$, then $\delta$ is the lowest root of $\Phi$ and $\Xi$ is the lowest weight of a standard $\mathfrak{g}_{0}^{(1)}$-module by Theorem 4.4. As this situation is easily seen to be impossible, $\delta \in \Phi^{+}$. Applying Theorem 4.4 (i), we conclude that $\delta=\alpha$ and $\Xi=-(\Lambda+\alpha)$. Thus, any $\mathfrak{b}^{-}$-primitive vector of $\mathfrak{g}_{1}$ has weight $-(\Lambda+\alpha)$ or $-\Lambda$.

Now if $e$ is any $\mathfrak{b}^{-}$-primitive vector from $\mathfrak{g}_{1}^{-\Lambda}$, then the tuple $\left(f^{\Lambda}, \mathfrak{g}_{0}, e\right)$ satisfies all the conditions of Theorem 3.17 (with $-\Delta$ being the set of simple roots). Hence $\left[f^{\Lambda},\left[e, f^{\Lambda}\right]\right] \neq 0$. But $\left[f^{\Lambda}, \mathfrak{g}_{1}^{-\Lambda}\right] \subseteq \mathfrak{t}$ and $\left[\mathfrak{t}, f^{\Lambda}\right] \subseteq \mathbb{F} f^{\Lambda}$, yielding $\left[f^{\Lambda},\left[e, f^{\Lambda}\right]\right] \in \mathbb{F} f^{\Lambda}$. From this it is immediate that the subspace of $\mathfrak{b}^{-}$primitive vectors from $\mathfrak{g}_{1}^{-\Lambda}$ has dimension $\leq 1$.

(d) Let $e^{-\Lambda}$ be a $\mathfrak{b}^{-}$-primitive vector in $\mathfrak{g}_{1}^{-\Lambda}$, and let $\widetilde{\mathfrak{g}}_{1}$ be the $\mathfrak{g}_{0}$-submodule of $\mathfrak{g}_{1}$ generated by $e^{-\Lambda}$. By our discussion in part (a), the subspace $\widetilde{\mathfrak{g}}_{1}$ is $G_{0}$-stable. Let $\widetilde{\mathfrak{g}}$ denote the graded subalgebra of $\mathfrak{g}$ generated by $f^{\Lambda}, \mathfrak{g}_{0}$, and $e^{-\Lambda}$. We claim that the graded Lie algebra $\widetilde{\mathfrak{g}} / \mathcal{M}(\widetilde{\mathfrak{g}})$ (where $\mathcal{M}(\widetilde{\mathfrak{g}})$ denotes the Weisfeiler radical) satisfies the hypotheses of Theorem 3.22. To prove the claim we need to show that the $\mathfrak{g}_{0}$-module $\widetilde{\mathfrak{g}}_{1}$ is irreducible.

Since the torus $T$ acts trivially on $\mathfrak{t}$, it preserves the subspace of $\mathfrak{b}^{-}$primitive vectors from $\widetilde{\mathfrak{g}}_{1}^{-\Lambda}$. By part (c), the latter is spanned by $e^{-\Lambda}$, so it must be that $e^{-\Lambda} \in \mathfrak{g}_{1}^{-\lambda}$ for some $-\lambda \in X\left(\mathfrak{g}_{1}\right)$. The equality $\widetilde{\mathfrak{g}}_{1}=\mathfrak{U}\left(\mathfrak{n}^{+}\right) e^{-\Lambda}$ implies that $-\lambda$ is a minimal weight of the $G_{0}$-module $\widetilde{\mathfrak{g}}_{1}$. But then $-w_{0} \lambda$ is 
a maximal weight of $\widetilde{\mathfrak{g}}_{1}$. Also, $\operatorname{dim}\left(\widetilde{\mathfrak{g}}_{1}\right)^{-w_{0} \lambda}=\operatorname{dim}\left(\widetilde{\mathfrak{g}}_{1}\right)^{-\lambda}=1$, by part (c). Since $\mathfrak{t} \cap \mathfrak{g}_{0}^{(1)}=\operatorname{Lie}(T)$, the restriction of $-w_{0} \Lambda$ to $\mathfrak{t} \cap \mathfrak{g}_{0}^{(1)}$ coincides with the differential of the rational character $-w_{0} \lambda$ at the identity element of $T$. From this it follows that the image of $-w_{0} \lambda$ in $X(T) / p X(T)$ coincides with

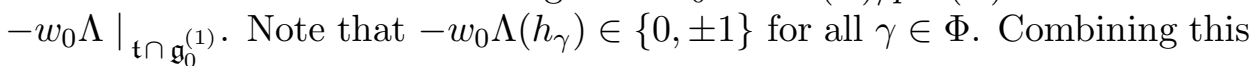
with (4.19) and (4.20) one derives that $-w_{0} \lambda=\varpi_{1}$. Since it also follows from (4.19) and 4.20) that $\nu \geq \varpi_{1}$ for all $\nu \in X_{+}\left(\mathfrak{g}_{1}\right)$, one obtains now that $\varpi_{1}$ is the only dominant weight of the $G_{0}$-module $\widetilde{\mathfrak{g}}_{1}$. As a consequence, all weights of the $G_{0}$-module $\widetilde{\mathfrak{g}}_{1}$ are conjugate under the Weyl group of $G_{0}$. Therefore, the $G_{0}$-modules $V$ and $\widetilde{\mathfrak{g}}_{1}$ are isomorphic. But then $\widetilde{\mathfrak{g}}_{1}$ is $\mathfrak{g}_{0}$-irreducible, hence the claim.

(e) The graded Lie algebra $\widetilde{\mathfrak{g}} / \mathcal{M}(\widetilde{\mathfrak{g}})$ (where $\mathcal{M}(\widetilde{\mathfrak{g}})$ denotes the Weisfeiler radical) satisfies the hypotheses of Theorem 3.22. Therefore by that theorem, this algebra is classical. Using [Bou1], it is easy to check that $\widetilde{\mathfrak{g}} / \mathcal{M}(\widetilde{\mathfrak{g}})$ corresponds to one of the following Dynkin diagrams:
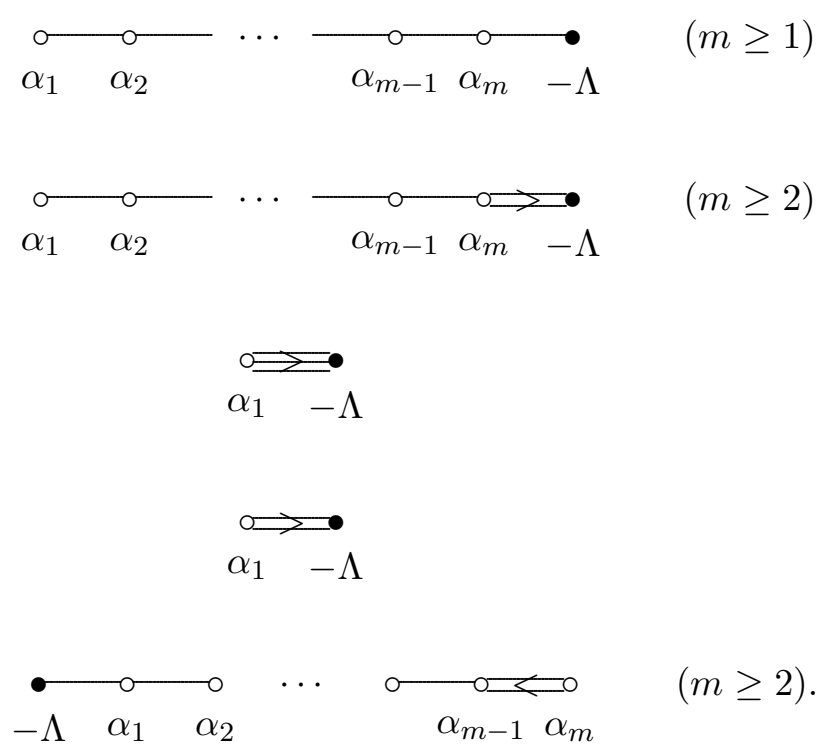

(Bear in mind that $\Phi$ is of type $\mathrm{A}_{m}, m \geq 2$, or $\mathrm{C}_{m}, m \geq 1$, and $\Lambda=\varpi_{m}$ if $\Phi=\mathrm{A}_{m}$ and $\Lambda=\varpi_{1}$ if $\Phi=\mathrm{C}_{m}$.) We may suppose that the vector $e^{-\Lambda}$ has been scaled if need be, so that $\left(f^{\Lambda}, h_{\Lambda}=\left[f^{\Lambda}, e^{-\Lambda}\right], e^{-\Lambda}\right)$ is a standard $\mathfrak{s l}_{2}$-triple.

If $\widetilde{\mathfrak{g}} / \mathcal{M}(\widetilde{\mathfrak{g}})$ has type $(4.22)$, set

$$
x_{\lambda}=\left[\left[\left[e_{-\alpha_{m}}, f^{\Lambda}\right], f^{\Lambda}\right], f^{\Lambda}\right] \quad \text { and } \quad x_{\gamma}=\left[\left[\left[e_{\alpha_{m}}, e^{-\Lambda}\right], e^{-\Lambda}\right], e^{\Gamma}\right],
$$

and use capital letters to denote the corresponding adjoint mappings. Then 


$$
\begin{aligned}
& {\left[x_{\lambda}, x_{\gamma}\right]=\left(\sum_{j=0}^{3}(-1)^{j}\left(\begin{array}{l}
3 \\
j
\end{array}\right)\left(F^{\Lambda}\right)^{j} E_{-\alpha_{m}}\left(F^{\Lambda}\right)^{3-j}\right)\left(\left[\left[\left[e_{\alpha_{m}}, e^{-\Lambda}\right], e^{-\Lambda}\right], e^{\Gamma}\right]\right)} \\
& \quad=\left(\Lambda-2 \alpha_{m}\right)\left(h_{\Lambda}\right)\left(\sum_{j=0}^{2}(-1)^{j}\left(\begin{array}{l}
3 \\
j
\end{array}\right)\left(F^{\Lambda}\right)^{j} E_{-\alpha_{m}}\left(F^{\Lambda}\right)^{2-j}\right)\left(\left[\left[e_{\alpha_{m}}, e^{-\Lambda}\right], e^{\Gamma}\right]\right) \\
& \quad=-2\left(3 \Lambda\left(h_{\alpha_{m}}\right) \Gamma\left(h_{\Lambda}\right)-3 \alpha_{m}\left(h_{\Lambda}\right) \Gamma\left(h_{\alpha_{m}}\right)-\alpha_{m}\left(h_{\Lambda}\right) \alpha\left(h_{\alpha_{m}}\right)\right) e_{-\alpha} \\
& \quad=-2(-12+12-2) e_{-\alpha}=4 e_{-\alpha} .
\end{aligned}
$$

As $\left[x_{\lambda}, e_{\alpha}\right]=0=\left[x_{\gamma}, e_{-\alpha}\right]$, the elements $x_{\lambda}, \frac{1}{4} x_{\gamma}, e_{-\alpha}$ satisfy the conditions of Lemma 4.3. But then $\lambda\left(h_{\alpha}\right) \in\{0,1\}$, which contradicts the fact that $\left(3 \Lambda-\alpha_{m}\right)\left(h_{\alpha}\right)=2$. So case $(4.22)$ cannot occur.

Now when $\widetilde{\mathfrak{g}} / \mathcal{M}(\widetilde{\mathfrak{g}})$ is of type $(4.23)$, set

$$
\begin{aligned}
& x_{\lambda}=\left[\left[\left[\left[e_{-\alpha_{1}}, f^{\Lambda}\right], f^{\Lambda}\right], f^{\Lambda}\right], f^{\Lambda}\right], \\
& x_{\gamma}=\left[\left[\left[\left[e_{\alpha_{1}}, e^{-\Lambda}\right], e^{-\Lambda}\right], e^{-\Lambda}\right], e^{\Gamma}\right] .
\end{aligned}
$$

Then, since here $\alpha_{1}\left(h_{\Lambda}\right)=3, \Lambda\left(h_{\alpha_{1}}\right)=1$, and $\left[f^{\Lambda}, e^{\Gamma}\right]=e_{-\alpha_{1}}$, we have

$$
\begin{aligned}
& {\left[x_{\lambda}, x_{\gamma}\right]=\left(\sum_{j=0}^{4}(-1)^{j}\left(\begin{array}{l}
4 \\
j
\end{array}\right)\left(F^{\Lambda}\right)^{j} E_{-\alpha_{1}}\left(F^{\Lambda}\right)^{4-j}\right)\left[\left[\left[\left[e_{\alpha_{1}}, e^{-\Lambda}\right], e^{-\Lambda}\right], e^{-\Lambda}\right], e^{\Gamma}\right]} \\
& =3\left(\Lambda-\alpha_{1}\right)\left(h_{\Lambda}\right)\left(\sum_{j=0}^{2}(-1)^{j}\left(\begin{array}{l}
4 \\
j
\end{array}\right)\left(F^{\Lambda}\right)^{j} E_{-\alpha_{1}}\left(F^{\Lambda}\right)^{3-j}\right)\left[\left[\left[e_{\alpha_{1}}, e^{-\Lambda}\right], e^{-\Lambda}\right], e^{\Gamma}\right] \\
& =-3\left(\Lambda-2 \alpha_{1}\right)\left(h_{\Lambda}\right)\left(\sum_{j=0}^{2}(-1)^{j}\left(\begin{array}{l}
4 \\
j
\end{array}\right)\left(F^{\Lambda}\right)^{j} E_{-\alpha_{1}}\left(F^{\Lambda}\right)^{2-j}\right)\left[\left[e_{\alpha_{1}}, e^{-\Lambda}\right], e^{\Gamma}\right] \\
& =12\left(6 \Lambda\left(h_{\alpha_{1}}\right) \Gamma\left(h_{\Lambda}\right)-4 \alpha_{1}\left(h_{\Lambda}\right) \Gamma\left(h_{\alpha_{1}}\right)\right. \\
& \left.\quad-\alpha_{1}\left(h_{\Lambda}\right) \alpha_{1}\left(h_{\alpha_{1}}\right)-\Lambda\left(h_{\alpha_{1}}\right) \alpha_{1}\left(h_{\Lambda}\right)\right) e_{-\alpha_{1}} \\
& =12(-30+36-6-3) e_{-\alpha_{1}}=-36 e_{-\alpha_{1}} .
\end{aligned}
$$

Since $\left[x_{\lambda}, e_{\alpha_{1}}\right]=0=\left[x_{\gamma}, e_{-\alpha_{1}}\right]$ and $\lambda\left(h_{\alpha_{1}}\right)=\left(4 \Lambda-\alpha_{1}\right)\left(h_{\alpha_{1}}\right)=2$, this case cannot occur by Lemma 4.3.

(f) Now suppose that $\Phi=\mathrm{A}_{m}$ for $m \geq 2$. Then $\widetilde{\mathfrak{g}} / \mathcal{M}(\widetilde{\mathfrak{g}})$ must be of type (4.21). As $\widetilde{\mathfrak{g}}_{-2}=\mathcal{M}(\widetilde{\mathfrak{g}})_{-2}$, it follows that $\left[\mathfrak{g}_{-2}, \widetilde{\mathfrak{g}}_{1}\right]=0$.

According to part (a), $\mathfrak{g}_{1}$ is a $G_{0}$-stable subspace of $V \otimes V \otimes V^{*}$. Obviously, $V \otimes V \otimes V^{*} \cong M \oplus N$ where $M \cong S^{2}(V) \otimes V^{*}$ and $N \cong \wedge^{2} V \otimes V^{*}$ as $\widetilde{G}_{0}$-modules. Since $\alpha=\varepsilon_{1}-\varepsilon_{m+1}, p>3$, and the set of weights of $N$ with respect to $\widetilde{T}$ equals $\left\{\varepsilon_{i}+\varepsilon_{j}-\varepsilon_{k} \mid 1 \leq i, j, k \leq m+1, i \neq j\right\}$, it is straightforward to see that the $\mathfrak{t}$-weight space $N^{-(\Lambda+\alpha)}=N^{\Gamma}$ is trivial (see the first 
paragraph of the proof). Therefore $\mathfrak{g}_{1}^{\prime}$, the $\mathfrak{g}_{0}$-submodule of $\mathfrak{g}_{1}$ generated by $e^{\Gamma}$, is contained in $M$. We claim that $\mathfrak{g}_{1}$ is isomorphic to a $\mathfrak{g}_{0}$-submodule of $M$.

If the natural projection map $\pi: \mathfrak{g}_{1} \rightarrow M$ is injective, there is nothing to prove. So we assume that $N \cap \mathfrak{g}_{1}=\operatorname{ker} \pi \neq 0$. If $M$ is an indecomposable $\mathfrak{g}_{0}^{(1)}$-module, then $\mathfrak{g}_{1}^{\prime}$ contains a $\mathfrak{g}_{0}^{(1)}$-submodule isomorphic to $V$ (see Theorem 2.69), hence it has a $\mathfrak{b}^{-}$-primitive vector of weight $-\Lambda$. Since $N \cap \mathfrak{g}_{1}$ has no nonzero weight vectors of weight $-(\Lambda+\alpha)$, it contains a nonzero $\mathfrak{b}^{-}$-primitive vector of weight $-\Lambda$; see part (c). But then the subspace of $\mathfrak{b}^{-}$-primitive vectors from $\mathfrak{g}_{1}^{-\Lambda}$ has dimension $\geq 2$, which is impossible by our final remark in part (c). Thus, $\mathfrak{g}_{1}^{\prime}$ is an irreducible $\mathfrak{g}_{0}^{(1)}$-module, and $M=\mathfrak{g}_{1}^{\prime} \oplus \widetilde{M}$, where $\widetilde{M}$ is a $\mathfrak{g}_{0}^{(1)}$-submodule of $M$ isomorphic to $V$ (see Theorem 2.69).

By part (a), the $\mathfrak{g}_{0}$-module $\operatorname{ker} \pi$ is $G_{0}$-stable. Assume $\mu$ is a minimal weight of $\operatorname{ker} \pi$, and let $(\operatorname{ker} \pi)^{\mu}$ be the corresponding weight space. As $(\operatorname{ker} \pi)^{\mu}$ lies in the subspace of fixed points of $N^{-}$, we have $\left[\mathfrak{n}^{-},(\operatorname{ker} \pi)^{\mu}\right]=0$. Thus, $(\operatorname{ker} \pi)^{\mu}$ consists of $\mathfrak{b}^{-}$-primitive vectors. Since ker $\pi \subseteq N$ contains no weight vector associated with $-(\Lambda+\alpha)$, the image of $\mu$ in $X(T) / p X(T)$ coincides with the restriction of $-\Lambda$ to $\mathfrak{t} \cap \mathfrak{g}_{0}^{(1)}$, and $\operatorname{dim}(\operatorname{ker} \pi)^{\mu}=1$. As $w_{0} \mu$ is dominant and $p>3$, it is immediate from (4.19) that $\mu=-\varpi_{m}$. Arguing as in part (d) we now deduce that all weights of the $G_{0}$-module $\operatorname{ker} \pi$ are conjugate under the Weyl group of $G_{0}$. It follows that the $G_{0}$-modules $V$ and $\operatorname{ker} \pi$ are isomorphic.

By the same reasoning one can show that $V \cong \pi^{-1}(\widetilde{M})$ as $G_{0}$-modules. As ker $\pi \subseteq \pi^{-1}(\widetilde{M})$, it must be that $\operatorname{ker} \pi=\pi^{-1}(\widetilde{M})$. Hence, $\pi\left(\mathfrak{g}_{1}\right)=\mathfrak{g}_{1}^{\prime}$. As $\mathfrak{g}_{1}^{\prime} \subseteq \mathfrak{g}_{1}$, this yields $\mathfrak{g}_{1}=\mathfrak{g}_{1}^{\prime} \oplus \operatorname{ker} \pi \cong \mathfrak{g}_{1}^{\prime} \oplus \widetilde{M}$, proving the claim.

If $\mathfrak{g}_{1}^{\prime}=\mathfrak{g}_{1}$ or if the $\mathfrak{g}_{0}^{(1)}$-submodule $S^{2}(V) \otimes V^{*}$ is completely reducible, then $\mathfrak{g}_{1}$ is generated by its $\mathfrak{b}^{-}$-primitive vectors. In other words, $\mathfrak{g}_{1}=$ $\mathfrak{g}_{1}^{\prime} \oplus \widetilde{\mathfrak{g}}_{1}$. (If $\mathfrak{g}_{1}$ has no $\mathfrak{b}^{-}$-primitive vectors of weight $-\Lambda$, we assume that $\widetilde{\mathfrak{g}}_{1}=0$.) Since we have proven that $\left[\mathfrak{g}_{-2}, \mathfrak{g}_{1}^{\prime}+\widetilde{\mathfrak{g}}_{1}\right]=0$, we must have $\mathfrak{g}_{-2}=0$ by 1-transitivity (1.3) in these cases. But then Theorem 2.66 applies showing that in the above cases $\mathfrak{g}$ is either $W(m+1 ; \underline{1})$ or $S(m+1 ; \underline{1})^{(1)}$ or $S(m+1 ; \underline{1})^{(1)} \oplus \mathbb{F} \mathfrak{D}_{1}$.

(g) Now suppose that $S^{2}(V) \otimes V^{*}$ is not completely reducible and $\mathfrak{g}_{1} \neq \mathfrak{g}_{1}^{\prime}$. As $\widetilde{\mathfrak{g}}_{1}$ is isomorphic to a maximal submodule of $S^{2}(V) \otimes V^{*}$, then $m+2 \equiv 0$ $\bmod p$ and $\mathfrak{g}_{1} \cong S^{2}(V) \otimes V^{*}$ (see Theorem 2.69). In particular, $\widetilde{\mathfrak{g}}_{1} \neq 0$. As $m+1 \not \equiv 0 \bmod p$, we have $\mathfrak{g l}(V)=\mathfrak{s l}(V) \oplus \mathbb{F} \mathrm{id}_{V}$. We regard $\operatorname{id}_{V}$ as the degree derivation and embed $\mathfrak{g}$ into the graded Lie algebra $\mathbb{F} \mathrm{id}_{V}+\mathfrak{g}$. Thus, we may assume in this part that $\mathfrak{g}_{0}=\mathfrak{g l}(V)$. Our nearest goal is to determine the bilinear mapping $V^{*} \times\left(S^{2}(V) \otimes V^{*}\right) \longrightarrow \mathfrak{g l}(V)$ given by the Lie bracket in $\mathfrak{g}$.

First let us show that $\operatorname{dim} \operatorname{Hom}_{\mathfrak{g}_{0}}\left(\mathfrak{g}_{-1} \otimes \mathfrak{g}_{1}, \mathfrak{g}_{0}\right)=3$. Clearly, 


$$
\begin{aligned}
\operatorname{Hom}_{\mathfrak{g}_{0}} & \left(\mathfrak{g}_{-1} \otimes \mathfrak{g}_{1}, \mathfrak{g}_{0}\right) \cong \operatorname{Hom}_{\mathfrak{g}_{0}}\left(V^{*} \otimes S^{2}(V) \otimes V^{*}, V \otimes V^{*}\right) \\
& \cong\left(V \otimes\left(S^{2}(V)\right)^{*} \otimes V \otimes V^{*} \otimes V\right)^{\mathfrak{g}_{0}} \\
& \cong\left(\left(S^{2}(V) \otimes V\right)^{*} \otimes V \otimes V \otimes V\right)^{\mathfrak{g}_{0}} \\
& \cong \operatorname{Hom}_{\mathfrak{g}_{0}}\left(S^{2}(V) \otimes V, V \otimes V \otimes V\right) \\
& \cong \operatorname{Hom}_{\mathfrak{g}_{0}}\left(S^{2}(V) \otimes V,\left(S^{2}(V) \oplus \wedge^{2}(V)\right) \otimes V\right) \\
& \cong \operatorname{Hom}_{\mathfrak{s}(V)}\left(V\left(2 \varpi_{1}\right) \otimes V\left(\varpi_{1}\right), V\left(2 \varpi_{1}\right) \otimes V\left(\varpi_{1}\right) \oplus V\left(\varpi_{2}\right) \otimes V\left(\varpi_{1}\right)\right),
\end{aligned}
$$

where $(\cdot)^{\mathfrak{g}_{0}}$ indicates the $\mathfrak{g}_{0}$-invariants. Since $m+1 \not \equiv 0 \bmod p$, the trace form $\Psi$ of the linear Lie algebra $\mathfrak{g l}(V)$ is nondegenerate on $\mathfrak{s l}(V)$. By [Bou2, Chap. VIII, Sec. 6], the Casimir element of $\mathfrak{U}(\mathfrak{s l}(V))$ associated with $\Psi$ acts on an $\mathfrak{s l}(V)$-module with highest weight $\lambda$ as the scalar $(\lambda, \lambda+2 \rho)$ where $\rho$ is the half-sum of the positive roots, and ( , ) is the corresponding $W$-invariant form on $\mathfrak{t}^{*}$. We may assume that

$$
\left(\mathfrak{t} \cap \mathfrak{g}_{0}^{(1)}\right)^{*}=\left\{\sum_{i=1}^{m+1} \lambda_{i} \varepsilon_{i} \mid \lambda_{1}+\cdots+\lambda_{m+1}=0\right\}
$$

where the $\lambda_{i} \in \mathbb{F}$, and where $\left(\varepsilon_{i}, \varepsilon_{j}\right)=\delta_{i, j}$ for $1 \leq i, j \leq m+1$. Then the fundamental weights are given by

$$
\varpi_{i}=\varepsilon_{1}+\cdots+\varepsilon_{i}-\frac{i}{m+1}\left(\varepsilon_{1}+\cdots+\varepsilon_{m+1}\right), \quad 1 \leq i \leq m .
$$

As $m+2 \equiv 0 \bmod p, \rho=\varpi_{1}+\cdots+\varpi_{m}=(m+1) \varepsilon_{1}+m \varepsilon_{2}+\cdots+2 \varepsilon_{m}+\varepsilon_{m+1}$, and so $\left(\varpi_{i}, \rho\right)=(m+1)+\cdots+(m-i+2)-\frac{i}{m+1}(1+2+\cdots+m+1)=$ $\frac{1}{2}((m+1)(m+2)-(m-i+1)(m-i+2)-i(m+2))$. Therefore, for $1 \leq i \leq j \leq m$, we have

$$
\begin{aligned}
\left(\varpi_{i}, 2 \rho\right) & =-i(i+1) \text { and } \\
\left(\varpi_{i}, \varpi_{j}\right) & =i-\frac{i j}{m+1}=i(j+1) .
\end{aligned}
$$

Using those values for $i=1,2,3$, it is easy to see the following:

\begin{tabular}{|c|c|c|c|}
\hline$\lambda$ & $3 \varpi_{1}$ & $\varpi_{1}+\varpi_{2}$ & $\varpi_{3}$ \\
\hline$(\lambda, \lambda+2 \rho)$ & 12 & 6 & 0 \\
\hline
\end{tabular}

Since the dominant weights of the $S L(V)$-modules $V\left(2 \varpi_{1}\right) \otimes V\left(\varpi_{1}\right), V\left(\varpi_{2}\right) \otimes$ $V\left(\varpi_{1}\right), V\left(3 \varpi_{1}\right), V\left(\varpi_{1}+\varpi_{2}\right)$, and $V\left(\varpi_{3}\right)$ belong to the set $\left\{3 \varpi_{1}, \varpi_{1}+\varpi_{2}\right.$, $\left.\varpi_{3}\right\}$, a standard argument shows that the Weyl modules $V\left(3 \varpi_{1}\right), V\left(\varpi_{1}\right.$ $\left.+\varpi_{2}\right)$, and $V\left(\varpi_{3}\right)$ are irreducible, and the tensor products $V\left(2 \varpi_{1}\right) \otimes V\left(\varpi_{1}\right)$ 
and $V\left(\varpi_{2}\right) \otimes V\left(\varpi_{1}\right)$ are completely reducible with

$$
\begin{gathered}
V\left(2 \varpi_{1}\right) \otimes V\left(\varpi_{1}\right) \cong V\left(3 \varpi_{1}\right) \oplus V\left(\varpi_{1}+\varpi_{2}\right) \\
V\left(\varpi_{2}\right) \otimes V\left(\varpi_{1}\right) \cong V\left(\varpi_{1}+\varpi_{2}\right) \oplus V\left(\varpi_{3}\right) .
\end{gathered}
$$

Thus, it follows from the above Casimir-element calculations that

$$
\begin{aligned}
& \operatorname{Hom}_{\mathfrak{g}_{0}}\left(\mathfrak{g}_{-1} \otimes \mathfrak{g}_{1}, \mathfrak{g}_{0}\right) \\
& \quad \cong \operatorname{Hom}_{\mathfrak{g r}(V)}\left(V\left(3 \varpi_{1}\right) \oplus V\left(\varpi_{1}+\varpi_{2}\right), V\left(3 \varpi_{1}\right) \oplus 2 V\left(\varpi_{1}+\varpi_{2}\right) \oplus V\left(\varpi_{3}\right)\right) \\
& \quad \cong \mathbb{F} \oplus \mathbb{F} \oplus \mathbb{F} .
\end{aligned}
$$

Let $v_{1}, \ldots, v_{m+1}$ be a basis for $V$ and let $v_{1}^{*}, \ldots, v_{m+1}^{*}$ be the dual basis in $V^{*}$. We use $\left\{v_{i} v_{j} \mid 1 \leq i, j \leq m+1\right\}$ as a basis for $S^{2}(V)$ and for brevity write $v_{i} v_{i}$ as $v_{i}^{2}$. We identify $E_{i, j} \in \mathfrak{g l}(V)$ with $v_{i} \otimes v_{j}^{*} \in V \otimes V^{*}$, so that $E_{i, j} v_{k}=v_{j}^{*}\left(v_{k}\right) v_{i}=\delta_{j, k} v_{i}$ and $E_{i, j} v_{k}^{*}=-v_{k}^{*}\left(v_{i}\right) v_{j}^{*}=-\delta_{i, k} v_{j}^{*}$. We define three maps $\zeta, \eta, \theta \in \operatorname{Hom}\left(V^{*} \otimes S^{2}(V) \otimes V^{*}, \mathfrak{g l}(V)\right)$ by

$$
\begin{gathered}
\zeta\left(v_{i}^{*} \otimes v_{j} v_{k} \otimes v_{\ell}^{*}\right)=\delta_{i, j} E_{k, \ell}+\delta_{i, k} E_{j, \ell} \\
\eta\left(v_{i}^{*} \otimes v_{j} v_{k} \otimes v_{\ell}^{*}\right)=\delta_{j, \ell} E_{k, i}+\delta_{k, \ell} E_{j, i} \\
\theta\left(v_{i}^{*} \otimes v_{j} v_{k} \otimes v_{\ell}^{*}\right)=\left(\delta_{i, j} \delta_{k, \ell}+\delta_{i, k} \delta_{j, \ell}\right) \mathrm{id}_{V} .
\end{gathered}
$$

Direct verification shows that these are $\mathfrak{g l}(V)$-module homomorphisms. It is evident that $\eta$ and $\theta$ are linearly independent, and since $\eta\left(v_{1}^{*} \otimes v_{1} v_{1} \otimes v_{2}^{*}\right)$ $=0=\theta\left(v_{1}^{*} \otimes v_{1} v_{1} \otimes v_{2}^{*}\right)$ and $\zeta\left(v_{1}^{*} \otimes v_{1} v_{1} \otimes v_{2}^{*}\right)=2 E_{1,2} \neq 0$, we have $\zeta \notin \mathbb{F} \eta$ $+\mathbb{F} \theta$. Therefore,

$$
\operatorname{Hom}_{\mathfrak{g l}(V)}\left(V^{*} \otimes S^{2}(V) \otimes V^{*}, \mathfrak{g l}(V)\right)=\mathbb{F} \zeta \oplus \mathbb{F} \eta \oplus \mathbb{F} \theta
$$

Let [, ] be the Lie bracket in $\mathfrak{g}$. We identify $\mathfrak{g}_{-1}, \mathfrak{g}_{0}, \mathfrak{g}_{1}$ with $V^{*}, \mathfrak{g l}(V)$, and $S^{2}(V) \otimes V^{*}$ respectively. Then $\left.[]\right|_{,\mathfrak{g}_{-1} \times \mathfrak{g}_{1}}=a \zeta+b \eta+c \theta$ for some $a, b, c \in \mathbb{F}$. Since $\mathfrak{g}$ is transitive, $a \neq 0$, as otherwise $\left[v_{i}^{*}, v_{1}^{2} \otimes v_{2}^{*}\right]=0$ for all $1 \leq i \leq m+1$. Furthermore,

$$
\begin{aligned}
{\left[\left[v_{i}^{*}, v_{n}^{*}\right], v_{j} v_{k} \otimes v_{\ell}^{*}\right]=} & {\left[v_{i}^{*},\left[v_{n}^{*}, v_{j} v_{k} \otimes v_{\ell}^{*}\right]-\left[v_{n}^{*},\left[v_{i}^{*}, v_{j} v_{k} \otimes v_{\ell}^{*}\right]\right.\right.} \\
= & {\left[v_{i}^{*}, a\left(\delta_{j, n} E_{k, \ell}+\delta_{k, n} E_{j, \ell}\right)+b\left(\delta_{j, \ell} E_{k, n}+\delta_{k, \ell} E_{j, n}\right)\right] } \\
+ & {\left[v_{i}^{*}, c\left(\delta_{j, n} \delta_{k, \ell}+\delta_{k, n} \delta_{j, \ell}\right) \mathrm{id}\right]-\left[v_{n}^{*}, a\left(\delta_{i, j} E_{k, \ell}+\delta_{i, k} E_{j, \ell}\right)\right] } \\
& -\left[v_{n}^{*}, b\left(\delta_{j, \ell} E_{k, i}+\delta_{k, \ell} E_{j, i}\right)+c\left(\delta_{i, j} \delta_{k, \ell}+\delta_{i, k} \delta_{j, \ell}\right) \mathrm{id}\right] \\
= & a\left(\delta_{j, n} \delta_{i, k}+\delta_{k, n} \delta_{i, j}\right) v_{\ell}^{*}+b\left(\delta_{j, \ell} \delta_{i, k}+\delta_{k, \ell} \delta_{i, j}\right) v_{n}^{*} \\
& +c\left(\delta_{j, n} \delta_{k, \ell}+\delta_{k, n} \delta_{j, \ell}\right) v_{i}^{*}-a\left(\delta_{i, j} \delta_{k, n}+\delta_{i, k} \delta_{j, n}\right) v_{\ell}^{*} \\
& -b\left(\delta_{j, \ell} \delta_{k, n}+\delta_{k, \ell} \delta_{j, n}\right) v_{i}^{*}-c\left(\delta_{i, j} \delta_{k, \ell}+\delta_{i, k} \delta_{j, \ell}\right) v_{n}^{*} \\
= & (b-c)\left(\left(\delta_{j, \ell} \delta_{i, k}+\delta_{k, \ell} \delta_{i, j}\right) v_{n}^{*}-\left(\delta_{j, \ell} \delta_{k, n}+\delta_{k, \ell} \delta_{j, n}\right) v_{i}^{*}\right)
\end{aligned}
$$


for $1 \leq i, j, k, \ell, n \leq m+1$. Suppose $\mathfrak{g}_{-2} \neq 0$. The 1-transitivity (1.3) of $\mathfrak{g}$ implies $\left[\mathfrak{g}_{-2}, \mathfrak{g}_{1}\right] \neq 0$, which forces $b-c \neq 0$. Thus, $a(b-c) \neq 0$. But then

$$
\begin{aligned}
{\left[\left[v_{m}^{*}, v_{m+1}^{*}\right],\left[v_{m}^{2} \otimes v_{m}^{*}, v_{m+1}^{2} \otimes v_{1}^{*}\right]\right] } & =\left[\left[\left[v_{m}^{*}, v_{m+1}^{*}\right], v_{m}^{2} \otimes v_{m}^{*}\right], v_{m+1}^{2} \otimes v_{1}^{*}\right] \\
& =2(b-c)\left[v_{m+1}^{*}, v_{m+1}^{2} \otimes v_{1}^{*}\right] \\
& =4 a(b-c) E_{m+1,1} \neq 0 .
\end{aligned}
$$

Set

$$
\begin{gathered}
\mathfrak{n}^{+}=\bigoplus_{1 \leq i<j \leq m+1} \mathbb{F} E_{i, j}, \quad \mathfrak{t}=\bigoplus_{1 \leq i \leq m+1} \mathbb{F} E_{i, i}, \quad \text { and } \\
\mathfrak{n}^{-}=\bigoplus_{1 \leq j<i \leq m+1} \mathbb{F} E_{i, j},
\end{gathered}
$$

and let $\mathfrak{b}^{+}=\mathfrak{t} \oplus \mathfrak{n}^{+}$. Clearly $u=\left[v_{m}^{*}, v_{m+1}^{*}\right]$ is a $\mathfrak{b}^{+}$-primitive vector of weight $-\left(\varepsilon_{m}+\varepsilon_{m+1}\right)$ in $\mathfrak{g}_{-2}$, and $\mathfrak{g}_{-2}=\mathfrak{U}\left(\mathfrak{n}^{-}\right)\left[v_{m}^{*}, v_{m+1}^{*}\right]$ because $\mathfrak{g}_{-2}=\left[\mathfrak{g}_{-1}, \mathfrak{g}_{-1}\right]$. Set $w=\left[v_{m}^{2} \otimes v_{m}^{*}, v_{m+1}^{2} \otimes v_{1}^{*}\right]$. As $\left[\mathfrak{n}^{-}, E_{m+1,1}\right]=0$, we have $\left[E_{i, j} u, w\right]$ $+\left[u, E_{i, j} w\right]=0$ for all $i>j$. If $k<m$, then

$$
\begin{aligned}
{\left[\left[v_{k}^{*}, v_{m+1}^{*}\right],\left[v_{m}^{2} \otimes v_{m}^{*}, v_{m+1}^{2} \otimes v_{1}^{*}\right]\right]=} & {\left[\left[\left[v_{k}^{*}, v_{m+1}^{*}\right], v_{m}^{2} \otimes v_{m}^{*}\right], v_{m+1}^{2} \otimes v_{1}^{*}\right] } \\
& +\left[v_{m}^{2} \otimes v_{m}^{*},\left[\left[v_{k}^{*}, v_{m+1}^{*}\right], v_{m+1}^{2} \otimes v_{1}^{*}\right]\right] \\
= & 0 .
\end{aligned}
$$

Similarly,

$$
\begin{aligned}
{\left[\left[v_{k}^{*}, v_{m}^{*}\right],\left[v_{m}^{2} \otimes v_{m}^{*}, v_{m+1}^{2} \otimes v_{1}^{*}\right]\right] } & =\left[\left[\left[v_{k}^{*}, v_{m}^{*}\right], v_{m}^{2} \otimes v_{m}^{*}\right], v_{m+1}^{2} \otimes v_{1}^{*}\right] \\
& =2(c-b)\left[v_{k}^{*}, v_{m+1}^{2} \otimes v_{1}^{*}\right]=0 .
\end{aligned}
$$

Since obviously, $\left[\left[v_{i}^{*}, v_{j}^{*}\right],\left[v_{m+1}^{2} \otimes v_{m}^{*}, v_{m+1}^{2} \otimes v_{1}^{*}\right]\right]=0$ for all $i, j \leq m-1$, we conclude that $\left[\left[\mathfrak{n}^{-}, \mathfrak{g}_{-2}\right], w\right]=0$. Inasmuch as $\left[\mathfrak{g}_{-2}, w\right]=\mathbb{F} E_{m+1,1}$ by the above computations, we have $\left[\mathfrak{g}_{-2},\left[\mathfrak{n}^{-}, w\right]\right]=\left[\left[\mathfrak{g}_{-2}, \mathfrak{n}^{-}\right], w\right]=0$.

Set $\mathfrak{g}_{2}^{\prime}:=\left\{x \in \mathfrak{g}_{2} \mid\left[\mathfrak{g}_{-2}, x\right]=0\right\}$. We have shown that $w \notin \mathfrak{g}_{2}^{\prime}$, and that $\left[\mathfrak{n}^{-}, w\right] \subseteq \mathfrak{g}_{2}^{\prime}$. Let $\mathfrak{g}^{\{2\}}$ be the graded subalgebra of $\mathfrak{g}$ generated by $\mathfrak{g}_{-2}, \mathfrak{g}_{0}$, and $\mathfrak{g}_{2}$. Let $M^{\{2\}}$ denote the maximal graded ideal of $\mathfrak{g}^{\{2\}}$ contained in the positive part $\bigoplus_{i>0} \mathfrak{g}_{i}^{\{2\}}$. Set $\overline{\mathfrak{g}}^{\{2\}}:=\mathfrak{g}^{\{2\}} / M^{\{2\}}$. Clearly, $\overline{\mathfrak{g}}^{\{2\}}$ is graded and transitive. It is easy to check that $M^{\{2\}} \cap \mathfrak{g}_{2}=\mathfrak{g}_{2}^{\prime}$. Hence, $\overline{\mathfrak{g}}_{1}^{\{2\}} \cong \mathfrak{g}_{2} / \mathfrak{g}_{2}^{\prime}$, $\overline{\mathfrak{g}_{0}}\{2\} \cong \mathfrak{g}_{0}$, and $\overline{\mathfrak{g}}_{-1}^{\{2\}} \cong \mathfrak{g}_{-2}$.

Let $\bar{w}$ denote the image of $w$ under the canonical epimorphism $\mathfrak{g}_{2} \rightarrow$ $\mathfrak{g}_{2} / \mathfrak{g}_{2}^{\prime}$. Since we know that $\bar{w}$ is a $\mathfrak{b}^{-}$-primitive vector of $\overline{\mathfrak{g}}_{1}^{\{2\}}$ and

$$
[u, \bar{w}] \in \mathbb{F}^{\times} e_{-\alpha}
$$

(here $\left.e_{-\alpha}=E_{m+1,1} \in \mathfrak{Z}\left(\mathfrak{n}^{-}\right)\right)$we identify $u$ with its image in $\overline{\mathfrak{g}}^{\{2\}}$. Since $u$ has weight $-\left(\varepsilon_{m}+\varepsilon_{m+1}\right)=\varepsilon_{1}+\cdots+\varepsilon_{m-1}=\varpi_{m-1}$ with respect to the torus $\mathfrak{t} \cap \mathfrak{g}_{0}^{(1)}$, the Lie algebra $\overline{\mathfrak{g}}^{\{2\}}$ is infinite-dimensional by Theorem 4.4. Therefore, $\mathfrak{g}_{-2}=0$, yielding $\left[\mathfrak{g}_{-1}, \mathfrak{g}_{-1}\right]=0$. (Note that in the case under consideration $m \geq 3$.) Thus, $\mathfrak{g}=\mathfrak{g}_{-1} \oplus \mathfrak{g}_{0} \oplus \mathfrak{g}_{1} \oplus \cdots \oplus \mathfrak{g}_{r}$, where $\mathfrak{g}_{0} \cong \mathfrak{g l}(V)$, 
and $\mathfrak{g}_{-1} \cong V^{*}$ as a $\mathfrak{g}_{0}$-module. Since $\mathfrak{g}$ is transitive, we apply Theorem 2.66 to conclude that $\mathfrak{g}$ is isomorphic to $W(m+1 ; \underline{1})$.

(h) It now remains for us to consider cases (4.21) for $m=1,(4.24)$ and (4.25). In other words, we may assume that $\mathfrak{g}_{0}^{(1)}=\mathfrak{s p}(V)$ and that $\mathfrak{g}_{-1} \cong V$ as a $\mathfrak{g}_{0}^{(1)}$-module. Recall from part (a) that $V \cong V^{*}$, and $\mathfrak{s p}(V) \cong S^{2}(V)$ as $\mathfrak{s p}(V)$-modules, and $\mathfrak{g}_{1}$ is a $\mathfrak{g}_{0}^{(1)}$-submodule of $V \oplus\left(V \otimes S^{2}(V)\right)$. We claim that $\mathfrak{g}_{1}$ is a completely reducible $\mathfrak{g}_{0}^{(1)}$-module.

First we recall that for any natural number $n$ there is an exact sequence, the Koszul resolution (see [Ja, p. 377]),

$$
\begin{aligned}
(4.26) \cdots \rightarrow S^{n-i}(V) \otimes \wedge^{i} V & \stackrel{\varphi_{i}}{\rightarrow} S^{n-i+1}(V) \otimes \wedge^{i-1} V \rightarrow \\
& \rightarrow \cdots \rightarrow S^{n-1}(V) \otimes V \rightarrow S^{n}(V) \rightarrow 0 .
\end{aligned}
$$

The map $\varphi_{i}$ is given by

$$
\varphi_{i}\left(x \otimes\left(v_{1} \wedge \cdots \wedge v_{i}\right)\right):=\sum_{j=1}^{i-1}(-1)^{i} x v_{j} \otimes\left(v_{1} \wedge \cdots \wedge \widehat{v}_{j} \wedge \cdots \wedge v_{i}\right)
$$

for all $v_{j} \in V$ and $x \in S^{n-i}(V)$. If $V$ is a module for a group $H$, then (4.26) is an exact sequence of $H$-modules. If $p$ does not divide $n$, then the exact sequence (4.26) splits. More precisely, define

by

$$
\psi_{j}: S^{j}(V) \otimes \wedge^{n-j} V \rightarrow S^{j-1}(V) \otimes \wedge^{n-j+1} V
$$

$$
\psi_{j}\left(w_{1} \cdots w_{j} \otimes w\right)=\sum_{i=1}^{j} w_{1} \cdots \widehat{w}_{i} \cdots w_{j} \otimes w_{i} \wedge w
$$

for all $w_{i} \in V$ and $w \in \wedge^{n-j} V$. Then all the $\psi_{j}$ are $H$-module homomorphisms, and

$$
\varphi_{i+1} \circ \psi_{n-i}+\psi_{n-i+1} \circ \varphi_{i}=m \cdot \operatorname{id}_{S^{n-i}(V) \otimes \wedge^{i} V}
$$

for all $i, 0 \leq i \leq n$. (We set $\varphi_{n+1}=\psi_{n+1}=0$.) If we set $n=3$ and $H=G_{0}=\operatorname{Sp}(V)$, we get that there is a split exact sequence of $G_{0}$-modules

$$
0 \rightarrow E \stackrel{\eta}{\rightarrow} S^{2}(V) \otimes V \rightarrow S^{3}(V) \rightarrow 0 .
$$

in which $E$ is a homomorphic image of $V \otimes \wedge^{2} V$ (as seen from (4.26) with $n=3)$. This implies that $S^{2}(V) \otimes V \cong S^{3}(V) \oplus E$.

According to part (a), $\mathfrak{g}_{1}$ is a $G_{0}$-stable subspace of $V \oplus\left(S^{2}(V) \otimes V\right)$. Thus, we may assume that $\mathfrak{g}_{1}$ is a $G_{0}$-submodule of $V \oplus E \oplus S^{3}(V)$. Let $\pi^{\prime}: \mathfrak{g}_{1} \rightarrow V \oplus E$ and $\pi^{\prime \prime}: \mathfrak{g}_{1} \rightarrow S^{3}(V)$ denote the corresponding projection maps, and put $U^{\prime}=\operatorname{ker} \pi^{\prime}$ and $U^{\prime \prime}=\operatorname{ker} \pi^{\prime \prime}$. If $U^{\prime \prime}=0$, then $\mathfrak{g}_{1}$ is isomorphic to a nonzero submodule of the $G_{0}$-module $S^{3}(V)$. Since $p>3$, Proposition 
2.77 implies that $S^{3}(V) \cong L\left(3 \varpi_{1}\right)$ is irreducible over $\mathfrak{g}_{0}^{(1)}$. As a consequence, $\mathfrak{g}_{1}$ is irreducible if $U^{\prime \prime}=0$.

Thus, in proving the claim we may assume that $U^{\prime \prime} \neq 0$. Note that

$$
X_{+}\left(V \otimes \wedge^{2} V\right)=\left\{\varpi_{1}+\varpi_{2}, \varpi_{3}, \varpi_{1}\right\},
$$

where $\varpi_{1}+\varpi_{2}$ is omitted if $m=1$ and $\varpi_{3}$ is omitted if $m=1,2$. Since $p>3$ and $\alpha=2 \varepsilon_{1}$, it is straightforward to see that the weight subspace $\left(V \otimes \wedge^{2} V\right)^{-\Lambda-\alpha}$ with respect to $\mathfrak{t}=\operatorname{Lie}(\widetilde{T})$ is trivial. Together with our remarks in part (c), this yields that $-\lambda=-\varpi_{1} \in X(T)$ is the only minimal weight of $U^{\prime \prime}$ and $\operatorname{dim}\left(U^{\prime \prime}\right)^{-\lambda}=1$. But then $-w_{0} \lambda=\varpi_{1}$ is the only maximal weight of $U^{\prime \prime}$. On the other hand, (4.20) says that $\nu \geq \varpi_{1}$ for any dominant weight $\nu$ of the $G_{0}$-module $\mathfrak{g}_{1}$. It follows that $\varpi_{1}$ is the only dominant weight of $U^{\prime \prime}$. Hence, $U^{\prime \prime} \cong L\left(\varpi_{1}\right) \cong V$ as $G_{0}$-modules.

Now since $e^{\Gamma} \in \mathfrak{g}_{1}$ and $\left(U^{\prime \prime}\right)^{\Gamma}=\left(U^{\prime \prime}\right)^{-\Lambda-\alpha}=0$, we have $\pi^{\prime \prime} \neq 0$. Hence, $\pi^{\prime \prime}\left(\mathfrak{g}_{1}\right)=S^{3}(V)$, thanks to Proposition 2.77. Therefore $\mathfrak{g}_{1}^{3 \varpi_{1}} \neq 0$. On the other hand, (4.27) shows that $\mathfrak{g}_{1}^{3 \varpi_{1}} \subseteq U^{\prime}$. Since $U^{\prime} \subseteq S^{3}(V)$, Proposition 2.77 implies that $U^{\prime} \cong \pi^{\prime \prime}\left(\mathfrak{g}_{1}\right)$. But then $\mathfrak{g}_{1} \cong V \oplus S^{3}(V)$ in the present case, to prove the claim.

(i) It follows from our discussion in part (h) that $\mathfrak{g}_{1}=\mathfrak{g}_{1}^{\prime} \oplus \widetilde{\mathfrak{g}}_{1}$, where we assume that $\widetilde{\mathfrak{g}}_{1}=0$ if $\mathfrak{g}_{1}$ has no $\mathfrak{b}^{-}$-primitive vectors of weight $-\Lambda$. If $\mathfrak{g}_{1}$ $=\mathfrak{g}_{1}^{\prime}$, then we have $\left[\mathfrak{g}_{-2}, \mathfrak{g}_{1}\right]$ by part (b), so that $\mathfrak{g}_{-2}=0$ by the 1-transitivity of $\mathfrak{g}$. Therefore, Theorem 2.66 applies, and we can conclude that $\mathfrak{g}$ is either $H(2 m ; \underline{1})^{(2)}$ or $H(2 m ; \underline{1})^{(2)} \oplus \mathbb{F} \mathfrak{D}_{1}$.

Thus, in what follows, we may assume that $\mathfrak{g}_{1}=\mathfrak{g}_{1}^{\prime} \oplus \widetilde{\mathfrak{g}}_{1}$, where $\widetilde{\mathfrak{g}}_{1} \cong V$ is generated by a nonzero $\mathfrak{b}^{-}$-primitive vector $e^{-\Lambda} \in \mathfrak{g}_{1}$. We have already established that $\left[\mathfrak{g}_{-2}, \mathfrak{g}_{1}^{\prime}\right]=0$. As before, denote by $\widetilde{\mathfrak{g}}$ the subalgebra generated by $f^{\Lambda}, \mathfrak{g}_{0}$, and $e^{-\Lambda}$. We know from part (e) that the Lie algebra $\widetilde{\mathfrak{g}} / \mathcal{M}(\widetilde{\mathfrak{g}})$ is either of type (4.24) or (4.25) or of type (4.21) for $m=1$. This implies that $\operatorname{dim} \mathfrak{g}_{-2} / \mathcal{M}(\widetilde{\mathfrak{g}})_{-2} \leq 1$ and $\mathfrak{g}_{-3}=\mathcal{M}(\widetilde{\mathfrak{g}})_{-3}$. Since $\left[\mathcal{M}(\widetilde{\mathfrak{g}})_{-2}, \widetilde{\mathfrak{g}}_{1}\right]=0=\left[\mathfrak{g}_{-2}, \mathfrak{g}_{1}^{\prime}\right]$ and $\mathfrak{g}_{1}=\mathfrak{g}_{1}^{\prime} \oplus \widetilde{\mathfrak{g}}_{1}$, we must have $\mathcal{M}(\widetilde{\mathfrak{g}})_{-2}=0$ by the 1 -transitivity of $\mathfrak{g}$. Hence, $\operatorname{dim} \mathfrak{g}_{-2} \leq 1$. If $\mathfrak{g}_{-3}=\left[\mathfrak{g}_{-2}, \mathfrak{g}_{-1}\right] \neq 0$, then the $\mathfrak{s p}(V)$-modules $\mathfrak{g}_{-3}$ and $\mathfrak{g}_{-1}$ are isomorphic. Moreover, because $\left[\mathfrak{g}_{-3}, \widetilde{\mathfrak{g}}_{1}\right] \subseteq \mathcal{M}(\widetilde{\mathfrak{g}})_{-2}=0$, it follows that $\left[\mathfrak{g}_{-3}, \mathfrak{g}_{1}^{\prime}\right]=\mathfrak{g}_{-2}$ by the 1-transitivity of $\mathfrak{g}$. But then $\mathfrak{g}_{1}^{\prime} \cong \mathfrak{g}_{-3}^{*}$ as $\mathfrak{g}_{0}^{(1)}$-modules, for both $\mathfrak{g}_{-3}$ and $\mathfrak{g}_{1}^{\prime}$ are irreducible. This, however, contradicts the fact that $\operatorname{dim} V<\operatorname{dim} S^{2}(V)$. Thus $\mathcal{M}(\widetilde{\mathfrak{g}})=0$; that is, $\operatorname{dim} \mathfrak{g}_{-2} \leq 1$, and $\mathfrak{g}_{-3}=0$. One can now apply Theorem 2.66 to conclude that either $\mathfrak{g} \cong K(2 m+1 ; \underline{1})^{(1)}$ or $\mathfrak{g} \cong W(2 ; \underline{1})$.

The proof of Theorem 4.18 is now finished.

\subsection{The irreducibility of $\mathfrak{g}_{1}$}

Here we investigate the case where $\left[f^{\Lambda}, e^{\Gamma}\right]=e_{\alpha}$ for some positive root $\alpha \in \Phi$. This case occurs for Lie algebras of type $S, C S, H$, or $C H$ when 
the natural grading is reversed, and our ultimate goal is to exclude all other possibilities. As a first step, we will show that $\mathfrak{g}_{1}$ is an irreducible $\mathfrak{g}_{0^{-}}$ module. Essential to the proof is the similarity between the structure of an irreducible restricted $\mathfrak{g}_{0}^{(1)}$-module and that of a rational module over the simply connected algebraic group corresponding to $\mathfrak{g}_{0}^{(1)}$.

Under the assumptions of the next three lemmas, $\mathfrak{g}_{0}^{(1)}$ is of type $\mathrm{A}_{m}$ or $\mathrm{C}_{m}$, and so $\mathfrak{g}_{0}^{(1)} \cong \mathfrak{s l}_{m+1}, \mathfrak{p s l}_{m+1}$, or $\mathfrak{s p}_{2 m}$. Given $\psi \in \mathfrak{t}^{*}$, we denote by $\bar{\psi}$ the restriction of $\psi$ to the subspace $\mathfrak{t} \cap \mathfrak{g}_{0}^{(1)}$. We adopt the notation of Section 2.3 .

LEMMA 4.28. Let $\mathfrak{g}$ be a graded Lie algebra satisfying conditions (1)-(4) and (6). Suppose there exist a $\mathfrak{b}^{+}$-primitive vector $f^{\Lambda} \in \mathfrak{g}_{-1}$ of weight $\Lambda$ and $a \mathfrak{b}^{-}$-primitive vector $e^{\Gamma} \in \mathfrak{g}_{1}$ of weight $\Gamma$ such that $\left[f^{\Lambda}, e^{\Gamma}\right]=e_{\alpha}$ for some root $\alpha \in \Phi^{+}$. Then $\mathfrak{g}_{0}^{(1)}$ consists of only one summand, which is of type $\mathrm{A}_{m}$ or $\mathrm{C}_{m}$, and $\mathfrak{g}_{1}$ is an irreducible standard $\mathfrak{g}_{0}^{(1)}$-module.

Proof. Replacing $\mathfrak{g}$ by its subalgebra $\widehat{\mathfrak{g}}$ generated by $\mathfrak{g}_{-1} \oplus \mathfrak{g}_{0} \oplus \mathfrak{g}_{1}$ and passing to the graded quotient $\widehat{\mathfrak{g}} / \mathcal{M}(\widehat{\mathfrak{g}})$, we can assume that $\mathfrak{g}$ is generated by its local part $\mathfrak{g}_{-1} \oplus \mathfrak{g}_{0} \oplus \mathfrak{g}_{1}$. By Proposition 4.14, $\Gamma\left(\mathfrak{t}^{[i]}\right) \neq 0$ for any $i=1, \ldots, \ell$. Then Theorem 4.4 applies to show $\mathfrak{g}_{0}^{(1)}$ has a unique summand which is of type $\mathrm{A}_{m}$ or $\mathrm{C}_{m} ; \alpha$ is the highest root; and $\Gamma$ is the lowest weight of an irreducible standard $\mathfrak{g}_{0}^{(1)}$-submodule of $\mathfrak{g}_{1}$. Thus, we have

$$
\begin{array}{cl}
\alpha=\varpi_{1}+\varpi_{m} ;-\bar{\Gamma} \in\left\{\varpi_{1}, \varpi_{m}\right\} ; \text { and } & \\
\bar{\Lambda}=2 \varpi_{1}+\varpi_{m} \text { or } \varpi_{1}+2 \varpi_{m} & \left(\mathrm{~A}_{m}\right) \\
\alpha=2 \varpi_{1} ;-\bar{\Gamma}=\varpi_{1} ; \text { and } \bar{\Lambda}=3 \varpi_{1} & \left(\mathrm{C}_{m}\right) .
\end{array}
$$

Let $V$ be an irreducible $\mathfrak{g}_{0}$-submodule of $\mathfrak{g}_{1}$. Since $\mathfrak{g}_{1}$ embeds into $\operatorname{Hom}\left(\mathfrak{g}_{-1}, \mathfrak{g}_{0}\right)$ by transitivity $(1.2)$, the $\mathfrak{g}_{0}^{(1)}$-module $V$ is restricted. Let $e=$ $e^{\Theta}$ be any $\mathfrak{b}^{-}$-primitive vector from $V$ of weight $\Theta \in \mathfrak{t}^{*}$. Then $V=\mathfrak{U}\left(\mathfrak{n}_{+}\right) e$. Since $\mathfrak{g}$ is irreducible and transitive, $\left[f^{\Lambda}, e\right] \neq 0$. Let $\widetilde{\mathfrak{g}}$ denote the subalgebra of $\mathfrak{g}$ generated by $f^{\Lambda}, \mathfrak{g}_{0}$, and $e$, and let $\mathcal{M}(\widetilde{\mathfrak{g}})$ be the Weisfeiler radical of $\widetilde{\mathfrak{g}}$.

If $\Theta \neq-\Lambda$, then Theorem 4.4 shows that $\Theta=\Gamma$. We suppose that $\Theta=-\Lambda$. Then the Lie algebra $\widetilde{\mathfrak{g}} / \mathcal{M}(\widetilde{\mathfrak{g}})$ is classical, and its grading is standard according to Theorem 3.22. If $\mathfrak{g}_{0}^{(1)}$ is of type $\mathrm{A}_{m}$ or $\mathrm{C}_{m}$, where $m \geq 2$, then using [Bou1] it is not difficult to verify that $\bar{\Lambda}$ is in the following list:

$$
\begin{array}{lr}
\left\{2 \varpi_{1}, \varpi_{1}, \varpi_{2}, \varpi_{m-1}, \varpi_{m}, 2 \varpi_{m}\right\}, & m \geq 8, \\
\left\{2 \varpi_{1}, \varpi_{1}, \varpi_{2}, \varpi_{3}, \varpi_{5}, \varpi_{6}, \varpi_{7}, 2 \varpi_{7}\right\}, & m=7, \\
\left\{\varpi_{i} \mid 1 \leq i \leq m\right\} \cup\left\{2 \varpi_{1}, 2 \varpi_{m}\right\}, & 2 \leq m \leq 6,
\end{array}
$$


for type $\mathrm{A}_{m}$, and

$$
\begin{array}{lr}
\left\{\varpi_{1}\right\}, & m \geq 4, \\
\left\{\varpi_{1}, \varpi_{m}\right\}, & m=2,3,
\end{array}
$$

for type $C_{m}$. But we have shown that $\bar{\Lambda}=2 \varpi_{1}+\varpi_{m}$ or $\bar{\Lambda}=\varpi_{1}+2 \varpi_{m}$ if $\mathfrak{g}_{0}^{(1)}$ is of type $A_{m}$, and $\bar{\Lambda}=3 \varpi_{1}$ if $\mathfrak{g}_{0}^{(1)}$ is of type $C_{m}$. These linear functions do not appear on the list. Hence, $\Theta \neq-\Lambda$ for $m \geq 2$.

If $\mathfrak{g}_{0}^{(1)}=\mathfrak{s l}_{2}$, then $\bar{\Lambda}=3 \varpi_{1}$. Suppose $\Theta=-\Lambda$. Then the classical Lie algebra $\widetilde{\mathfrak{g}} / \mathcal{M}(\widetilde{\mathfrak{g}})$ has the following Dynkin diagram of type $\mathrm{G}_{2}$ :

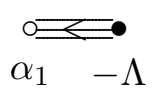

Set $f^{\prime}=\left[f^{\Lambda},\left[f^{\Lambda}, e_{-\alpha_{1}}\right]\right]$ and $e^{\prime}=\left[e^{\Gamma}, e^{-\Lambda}\right]$. Then

$$
\begin{aligned}
{\left[f^{\prime}, e^{\prime}\right] } & =\left(\left(F^{\Lambda}\right)^{2} E_{-\alpha_{1}}-2 F^{\Lambda} E_{-\alpha_{1}} F^{\Lambda}+E_{-\alpha_{1}}\left(F^{\Lambda}\right)^{2}\right)\left(\left[e^{\Gamma}, e^{-\Lambda}\right]\right) \\
& =\left(-2 F^{\Lambda} E_{-\alpha_{1}}+E_{-\alpha_{1}} F^{\Lambda}\right)\left(\left[e_{\alpha_{1}}, e^{-\Lambda}\right]+\left[h_{-\Lambda}, e^{\Gamma}\right]\right) \\
& =2 \Lambda\left(h_{\alpha_{1}}\right) h_{-\Lambda}-\left(\alpha_{1}\left(h_{-\Lambda}\right)+2+\alpha_{1}\left(h_{-\Lambda}\right)\right) h_{\alpha_{1}} \\
& =6 h_{-\Lambda} .
\end{aligned}
$$

Here $F^{\Lambda}=\operatorname{ad} f^{\Lambda}, E_{-\alpha_{1}}=\operatorname{ad} e_{-\alpha_{1}}$, and we assume that

$$
\left[e^{-\Lambda}, f^{\Lambda}\right]=h_{-\Lambda}, \quad\left[h_{-\Lambda}, e^{-\Lambda}\right]=2 e^{-\Lambda}, \quad \text { and } \quad\left[h_{-\Lambda}, f^{\Lambda}\right]=-2 f^{\Lambda} .
$$

Moreover, $\left(2 \Lambda-\alpha_{1}\right)\left(h_{\alpha_{1}}\right)=4$ and $\left[\mathfrak{n}^{+}, f^{\prime}\right]=0=\left[\mathfrak{n}^{-}, e^{\prime}\right]$. Setting

$$
\begin{array}{ll}
e_{1}=e_{\alpha_{1}}, & f_{1}=e_{-\alpha_{1}}, \\
e_{2}=\frac{1}{3}\left[e^{\Gamma}, e^{-\Lambda}\right], & f_{2}=-\frac{1}{3}\left[f^{\Lambda},\left[f^{\Lambda}, e_{-\alpha_{1}}\right]\right], \\
h_{1}=h_{\alpha_{1}}, & h_{2}=\frac{2}{3} h_{-\Lambda},
\end{array}
$$

we have from the calculations in the previous paragraph,

$$
\begin{array}{lll}
{\left[e_{i}, f_{j}\right]=\delta_{i, j} h_{i},} & {\left[h_{i}, h_{j}\right]=0,} \\
{\left[h_{i}, e_{j}\right]=A_{i, j} e_{j},} & {\left[h_{i}, f_{j}\right]=-A_{i, j} f_{j}} & (1 \leq i, j \leq 2),
\end{array}
$$

where $A=\left(A_{i, j}\right)$ is the matrix

$$
A=\left(\begin{array}{cc}
2 & -4 \\
-\frac{2}{3} & 2
\end{array}\right)
$$


Now put $e_{1}^{\prime}:=\left[e_{1},\left[e_{1}, e_{2}\right]\right], f_{1}^{\prime}:=\left[f_{1},\left[f_{1}, f_{2}\right]\right], u_{1}:=\left[e_{1},\left[e_{1},\left[e_{1}, e_{2}\right]\right]\right]$, and $v_{1}:=\left[f_{1},\left[f_{1},\left[f_{1}, f_{2}\right]\right]\right]$. Then

$$
\begin{aligned}
{\left[e_{1}^{\prime}, f_{1}^{\prime}\right] } & =\left[\left[e_{1},\left[e_{1}, e_{2}\right]\right],\left[f_{1},\left[f_{1}, f_{2}\right]\right]\right] \\
& =\left(E_{1}^{2} E_{2}-2 E_{1} E_{2} E_{1}+E_{2} E_{1}^{2}\right)\left(\left[f_{1},\left[f_{1}, f_{2}\right]\right]\right) \\
& =\left(-2 E_{1} E_{2}+E_{2} E_{1}\right)\left(6\left[f_{1}, f_{2}\right]\right) \\
& =6\left(-2 E_{1}\left(-\frac{2}{3} f_{1}\right)+E_{2}\left(4 f_{2}\right)\right) \\
& =8\left(h_{1}+3 h_{2}\right) .
\end{aligned}
$$

Also, $\left[\left[e_{1}, e_{2}\right],\left[f_{1}, f_{2}\right]\right]=\left[e_{1},\left[f_{1}, h_{2}\right]\right]-\left[e_{2},\left[h_{1}, f_{2}\right]\right]=-\frac{2}{3} h_{1}-4 h_{2}$, and

$$
\begin{aligned}
{\left[u_{1}, v_{1}\right] } & =\left[\left[e_{1},\left[e_{1},\left[e_{1} e_{2}\right]\right]\right],\left[f_{1},\left[f_{1},\left[f_{1} f_{2}\right]\right]\right]\right] \\
& =\left(E_{1}^{3} E_{2}-3 E_{1}^{2} E_{2} E_{1}+3 E_{1} E_{2} E_{1}^{2}-E_{2} E_{1}^{3}\right)\left(\left[f_{1},\left[f_{1},\left[f_{1}, f_{2}\right]\right]\right]\right) \\
& =\left(-3 E_{1}^{2} E_{2}+3 E_{1} E_{2} E_{1}-E_{2} E_{1}^{2}\right)\left(6\left[f_{1},\left[f_{1} f_{2}\right]\right]\right) \\
& =\left(3 E_{1} E_{2}-E_{2} E_{1}\right)\left(36\left[f_{1} f_{2}\right]\right) \\
& =36\left(3 E_{1}\left(-\frac{2}{3} f_{1}\right)-E_{2}\left(4 f_{2}\right)\right) \\
& =-72\left(h_{1}+2 h_{2}\right) .
\end{aligned}
$$

Note that $\left[-\frac{2}{3} h_{1}-4 h_{2}, e_{1}\right]=\frac{4}{3} e_{1},\left[-\frac{2}{3} h_{1}-4 h_{2}, e_{2}\right]=-\frac{16}{3} e_{2},\left[h_{1}+2 h_{2}, e_{1}\right]=$ $\frac{2}{3} e_{1}$, and $\left[h_{1}+2 h_{2}, e_{2}\right]=0$. Now put $e_{1}^{\prime \prime}:=\left[\left[e_{1}, e_{2}\right], u_{1}\right]$ and $f_{1}^{\prime \prime}:=\left[\left[f_{1}, f_{2}\right], v_{1}\right]$. Since $\left[\left[e_{1}, e_{2}\right], v_{1}\right]=\left[\left[f_{1}, f_{2}\right], u_{1}\right]=0$, we have that

$$
\begin{aligned}
{\left[e_{1}^{\prime \prime}, f_{1}^{\prime \prime}\right] } & =\left[\left[\left[e_{1}, e_{2}\right], u_{1}\right],\left[\left[f_{1}, f_{2}\right], v_{1}\right]\right] \\
& =\left[\left[e_{1}, e_{2}\right],\left[\left[f_{1}, f_{2}\right],\left[u_{1}, v_{1}\right]\right]\right]-\left[u_{1},\left[\left[\left[e_{1}, e_{2}\right],\left[f_{1}, f_{2}\right]\right], v_{1}\right]\right] \\
& =\left[\left[e_{1}, e_{2}\right],\left[\left[f_{1}, f_{2}\right],-72\left(h_{1}+2 h_{2}\right)\right]\right]-\left[u_{1},\left[-\frac{2}{3} h_{1}-4 h_{2}, v_{1}\right]\right] \\
& =72\left[\left[e_{1}, e_{2}\right],-\frac{2}{3}\left[f_{1}, f_{2}\right]\right]-\left[u_{1}, \frac{4}{3} v_{1}\right] \\
& =-48\left(-\frac{2}{3} h_{1}-4 h_{2}\right)-\frac{4}{3}\left(-72\left(h_{1}+2 h_{2}\right)\right) \\
& =128\left(h_{1}+3 h_{2}\right) .
\end{aligned}
$$

Next observe that

$$
\begin{aligned}
{\left[e_{1}^{\prime}, f_{1}^{\prime \prime}\right]=} & {\left[\left[e_{1},\left[e_{1}, e_{2}\right]\right],\left[\left[f_{1}, f_{2}\right], v_{1}\right]\right] } \\
= & {\left[\left[\left[e_{1},\left[e_{1}, e_{2}\right]\right],\left[f_{1}, f_{2}\right]\right], v_{1}\right] } \\
& \quad+\left[\left[f_{1}, f_{2}\right],\left[\left[e_{1},\left[e_{1}, e_{2}\right]\right],\left[f_{1},\left[f_{1},\left[f_{1}, f_{2}\right]\right]\right]\right]\right] \\
= & {\left[\left[\left[\left[e_{1},\left[e_{1}, e_{2}\right]\right], f_{1}\right], f_{2}\right], v_{1}\right]+\left[\left[f_{1}, f_{2}\right],\left[\left[e_{1}^{\prime}, f_{1}\right], f_{1}^{\prime}\right]+\left[f_{1},\left[e_{1}^{\prime}, f_{1}^{\prime}\right]\right]\right] }
\end{aligned}
$$

so that 


$$
\begin{aligned}
{\left[e_{1}^{\prime}, f_{1}^{\prime \prime}\right]=} & -6\left[\left[\left[e_{1}, e_{2}\right], f_{2}\right], v_{1}\right] \\
& \left.\quad+\left[\left[f_{1}, f_{2}\right],\left[\left[e_{1},\left[e_{1}, e_{2}\right]\right], f_{1}\right], f_{1}^{\prime}\right]+8\left[f_{1}, h_{1}+3 h_{2}\right]\right] \\
= & -6\left[\left[e_{1}, h_{2}\right], v_{1}\right]-6\left[\left[f_{1}, f_{2}\right],\left[\left[e_{1}, e_{2}\right], f_{1}^{\prime}\right]\right] \\
= & -4\left[e_{1},\left[f_{1},\left[f_{1},\left[f_{1}, f_{2}\right]\right]\right]\right]+6\left[\left[f_{1}, f_{2}\right],\left[e_{2},\left[e_{1},\left[f_{1},\left[f_{1}, f_{2}\right]\right]\right]\right]\right] \\
= & -24\left[f_{1},\left[f_{1}, f_{2}\right]\right]+36\left[\left[f_{1}, f_{2}\right],\left[e_{2},\left[f_{1}, f_{2}\right]\right]\right] \\
= & -24\left[f_{1},\left[f_{1}, f_{2}\right]\right]+36\left[\left[f_{1}, f_{2}\right],\left[f_{1}, h_{2}\right]\right] \\
= & -24\left[f_{1},\left[f_{1}, f_{2}\right]\right]-24\left[\left[f_{1}, f_{2}\right], f_{1}\right]=0 .
\end{aligned}
$$

Similarly, $\left[e_{1}^{\prime \prime}, f_{1}^{\prime}\right]=0$. As $\left[h_{1}+3 h_{2}, e_{1}^{\prime}\right]=4 e_{1}^{\prime} \neq 0$ and $\left[h_{1}+3 h_{2}, e_{1}^{\prime \prime}\right]=8 e_{1}^{\prime \prime} \neq$ 0 , Theorem 3.8 now implies that $e_{1}^{\prime}, f_{1}^{\prime}, e_{1}^{\prime \prime}$, and $f_{1}^{\prime \prime}$ generate an infinitedimensional Lie algebra. Since $\mathfrak{g}$ is finite-dimensional, we conclude that $\Theta=\Gamma$.

We let $-\Delta$ be the base of the root system $\Phi$ and reverse the grading of $\widetilde{\mathfrak{g}} / \mathcal{M}(\widetilde{\mathfrak{g}})$, so that $(\widetilde{\mathfrak{g}} / \mathcal{M}(\widetilde{\mathfrak{g}}))_{i}=\widetilde{\mathfrak{g}}_{-i} / \mathcal{M}(\widetilde{\mathfrak{g}})_{-i}$ for all $i$. Note that $(\widetilde{\mathfrak{g}} / \mathcal{M}(\widetilde{\mathfrak{g}}))_{-1} \cong$ $V$ and $(\tilde{\mathfrak{g}} / \mathcal{M}(\widetilde{\mathfrak{g}}))_{1} \cong \mathfrak{g}_{-1}$ as $\mathfrak{g}_{0}$-modules. Then it is straightforward to see that the Lie algebra $\widetilde{\mathfrak{g}} / \mathcal{M}(\widetilde{\mathfrak{g}})$ (viewed with its new grading) satisfies all the conditions of Theorem 4.18. Since $(\widetilde{\mathfrak{g}} / \mathcal{M}(\widetilde{\mathfrak{g}}))_{1}$ is an irreducible $\mathfrak{g}_{0}$-module, Theorem 2.69 now shows that if $\mathfrak{g}_{0}^{(1)} \cong \mathfrak{s l}_{m+1}, m \geq 1$, then $m+2 \not \equiv 0$ $\bmod p$.

As $\mathfrak{g}_{0}^{(1)}$ is almost simple and $\mathfrak{Z}\left(\mathfrak{g}_{0}\right)$ acts faithfully on $\mathfrak{g}_{1}$, we may assume that $\mathfrak{g}_{0} \subseteq \mathfrak{g l}(V)$. When $\mathfrak{g}_{0}^{(1)}$ is of type $\mathrm{A}_{m}$, then $\mathfrak{s l}(V) \subseteq \mathfrak{g}_{0} \subseteq \mathfrak{g l}(V)$, and when $\mathfrak{g}_{0}^{(1)}$ is of type $\mathrm{C}_{m}$, then $\mathfrak{s p}(V) \subseteq \mathfrak{g}_{0} \subseteq \mathfrak{c} \mathfrak{s p}(V)$. Let $G_{0}, T$, and $N^{ \pm}$be the algebraic groups introduced at the beginning of the proof of Theorem 4.18. To simplify the notation, we will identify the weights $\nu \in X_{1}(T)$ with their differentials $(\mathrm{d} \nu)_{e} \in\left(\mathfrak{t} \cap \mathfrak{g}_{0}^{(1)}\right)^{*} \cong X(T) \otimes_{\mathbb{Z}} \mathbb{F}$. Since $G_{0}$ is a simply connected group and $\mathfrak{g}_{-1}$ is an irreducible restricted $\mathfrak{g}_{0}^{(1)}$-module, there is a unique $\lambda \in X_{1}(T)$ such that $\mathfrak{g}_{-1} \cong L(\lambda)$ as $\mathfrak{g}_{0}^{(1)}$-modules, where the action of $\mathfrak{g}_{0}^{(1)}$ on $L(\lambda)$ is induced by the differential of the rational action of $G_{0}$ on $L(\lambda)$; see Proposition 2.13. The image of $\lambda$ in $X(T) / p X(T) \hookrightarrow\left(\mathfrak{t} \cap \mathfrak{g}_{0}^{(1)}\right)^{*}$ equals $\bar{\Lambda}$.

From now on we will identify $\mathfrak{g}_{-1}$ with $L(\lambda)$. The group $G_{0}$ acts on $\mathfrak{g}_{0}$ by conjugation, and hence it acts rationally on the vector space $\operatorname{Hom}\left(\mathfrak{g}_{-1}, \mathfrak{g}_{0}\right) \cong$ $\mathfrak{g}_{-1}^{*} \otimes \mathfrak{g}_{0}$. By the choice of $L(\lambda)$, the differential of this action coincides with the natural action of $\mathfrak{g}_{0}^{(1)}$ on $\operatorname{Hom}\left(\mathfrak{g}_{-1}, \mathfrak{g}_{0}\right)$.

First we suppose that $\mathfrak{g}_{0}^{(1)}$ is of type $A_{m}$ for $m \geq 2$. Renumbering the simple roots if necessary, we may assume that $\bar{\Gamma}=-\varpi_{m}$ (see the proof of Theorem 4.18 for a similar argument). Then $\mathfrak{g}_{-1}=L\left(\varpi_{1}+2 \varpi_{m}\right)$. Since $w_{0} \varpi_{i}=-\varpi_{m+1-i}, 1 \leq i \leq m$, we have that $V \cong L\left(\varpi_{1}\right)$, and $\mathfrak{g}_{-1}^{*} \cong$ $L\left(2 \varpi_{1}+\varpi_{m}\right)$ as $G_{0}$-modules; see Proposition 2.12. From this it follows that 
$\mathfrak{g}_{-1}^{*}$ is isomorphic to a composition factor of the $G_{0}$-module $S^{2}(V) \otimes V^{*}$. Because the $G_{0}$-module $\mathfrak{g}_{0}$ is isomorphic to a $G_{0}$-submodule of $V \otimes V^{*}$, any weight of $\operatorname{Hom}\left(\mathfrak{g}_{-1}, \mathfrak{g}_{0}\right)$ relative $T$ belongs to the set

$$
R:=\left\{\varepsilon_{i_{1}}+\varepsilon_{i_{2}}+\varepsilon_{i_{3}}-\varepsilon_{j_{1}}-\varepsilon_{j_{2}} \mid 1 \leq i_{1}, i_{2}, i_{3}, j_{1}, j_{2} \leq m+1\right\} .
$$

Since $-\varepsilon_{m+1}=\varpi_{m}$ and $-\varepsilon_{m}-\varepsilon_{m+1}=\varpi_{m-1}$ as rational characters of $T$ and since the Weyl group $W$ of $G_{0}$ permutes the $\varepsilon_{i}$ 's, we have that

$$
\begin{aligned}
R \cap X(T)_{+}=\{ & 3 \varpi_{1}+2 \varpi_{m}, 3 \varpi_{1}+\varpi_{m-1}, 2 \varpi_{1}+\varpi_{m}, \\
& \varpi_{1}+\varpi_{2}+2 \varpi_{m}, \varpi_{3}+2 \varpi_{m}, \varpi_{1}+\varpi_{2}+\varpi_{m-1}, \\
& \left.\varpi_{3}+\varpi_{m-1}, \varpi_{2}+\varpi_{m}, \varpi_{1}\right\},
\end{aligned}
$$

where $\varpi_{3}+\varpi_{m-1}$ is omitted if $m \leq 3$, and $\varpi_{1}+\varpi_{2}+\varpi_{m-1}, \varpi_{3}+2 \varpi_{m}$ are omitted if $m=2$. Clearly, $R \cap X(T)_{+} \subset X_{1}(T)$. But then $\mathfrak{g}_{1}$ is a $G_{0}$-stable subspace of $\operatorname{Hom}\left(\mathfrak{g}_{-1}, \mathfrak{g}_{0}\right)$ by Proposition 2.15 .

Let $\mu$ be a minimal weight of the $G_{0}$-module $\mathfrak{g}_{1}$, and let $\mathfrak{g}_{1}^{\mu}$ denote the corresponding weight space. Since $\mathfrak{g}_{1}^{\mu}$ is contained in the subspace of fixed points of the subgroup $N^{-}$, we have $\left[\mathfrak{n}^{-}, \mathfrak{g}_{1}^{\mu}\right]=0$. As $T$ acts as the identity on $\mathfrak{t}$, the weight space $\mathfrak{g}_{1}^{\mu}$ is $\mathfrak{t}$-stable. Let $e_{1}=e^{\Theta_{1}}$ be any nonzero weight vector for $\mathfrak{t}$ contained in $\mathfrak{g}_{1}^{\mu}$. It follows that $e_{1}$ is a $\mathfrak{b}^{-}$-primitive vector, and its weight $\bar{\Theta}_{1}$ coincides with the image of $\mu$ in $X(T) / p X(T) \hookrightarrow\left(\mathfrak{t} \cap \mathfrak{g}_{0}^{(1)}\right)^{*}$. If $\Theta_{1} \neq \Gamma$, then $\Theta_{1}=-\Lambda$ by Theorem 4.4 (as in part (c) of the proof of Theorem 4.18).

Suppose $\Theta_{1}=-\Lambda$. Since $w_{0} \mu$ is a maximal weight of $\mathfrak{g}_{1}$, it belongs to $R \cap X(T)_{+}$. On the other hand, the image of $w_{0} \mu$ in $X(T) / p X(T)$ equals $w_{0} \bar{\Lambda}$. As $p>3$, this implies that $w_{0} \mu=2 \varpi_{1}+\varpi_{m}$. Let $M$ denote the $G_{0}$-submodule of $\mathfrak{g}_{1}$ generated by $e_{1}$. The orbit $N_{G_{0}}(T) \cdot e_{1}$ contains an eigenvector for $B^{+}=T N^{+}$of weight $w_{0} \mu$. Therefore, $M$ is a homomorphic image of the Weyl module $V\left(2 \varpi_{1}+\varpi_{m}\right)$; see Proposition 2.14 (a). Combining Theorem 2.69 with Weyl's dimension formula (2.11), it is now easy to observe that

$$
\operatorname{dim} V\left(2 \varpi_{1}+\varpi_{m}\right)=\operatorname{dim}\left(S^{2}(V) \otimes V^{*}\right)-\operatorname{dim} V^{*}=\operatorname{dim} W(m+1)_{1}^{\dagger},
$$

where $W(m+1)_{1}^{\dagger}$ is as in $(2.70)$. Since $m+2 \not \equiv 0 \bmod p$ by our earlier remarks, Theorem 2.69 says that the $\mathfrak{s l}_{m+1}$-module $W(m+1)_{1}^{\dagger}$ is irreducible and contains a $\mathfrak{b}^{+}$-primitive vector of weight $2 \varpi_{1}+\varpi_{m}$. But then Proposition 2.13 yields $\operatorname{dim} L\left(2 \varpi_{1}+\varpi_{m}\right)=\operatorname{dim} W(m+1)_{1}^{\dagger}$. It follows that $V\left(2 \varpi_{1}+\varpi_{m}\right) \cong L\left(2 \varpi_{1}+\varpi_{m}\right) \cong M$. As a result, $M$ is an irreducible $\mathfrak{g}_{0^{-}}$ module. Hence, we can replace the triple $\left(f^{\Lambda}, \mathfrak{g}_{0}, e\right)$ by the triple $\left(f^{\Lambda}, \mathfrak{g}_{0}, e_{1}\right)$ and argue as before to conclude that $\Theta_{1} \neq-\Lambda$. As a consequence, $\mathfrak{g}_{1}^{\mu} \subseteq \mathfrak{g}_{1}^{\Gamma}$; that is, the image of $\mu$ in $X(T) / p X(T)$ coincides with $\Gamma$.

From the description of $R$ above and the fact that $p>3$, we see that $\mu=-\varpi_{m}$. There is a linear function $\delta \in\left(\mathfrak{g}_{1}^{\mu}\right)^{*}$ such that $\left[f^{\Lambda}, x\right]=\delta(x) e_{\alpha}$ for any $x \in \mathfrak{g}_{1}^{\mu}$. Since $\mathfrak{g}$ is irreducible and transitive, and $\mathfrak{g}_{1}^{\mu}$ consists of 
$\mathfrak{b}^{-}$-primitive vectors, we have $\left[f^{\Lambda}, x\right] \neq 0$ whenever $0 \neq x \in \mathfrak{g}_{1}^{\mu}$. This yields $\operatorname{ker} \delta=0$, i.e., $\operatorname{dim} \mathfrak{g}_{1}^{\mu}=1$. If $\nu$ is a weight of the $G_{0}$-module $\mathfrak{g}_{-1}^{*} \otimes \mathfrak{g}_{0}$, then $w(\nu) \in \Delta \cap X(T)_{+}$for some $w \in W$. Using the list of weights in $R \cap X(T)_{+}$ given above, it is easy to see that $w(\nu) \geq \varpi_{1}$. But then $w_{0} w(\nu) \leq w_{0}\left(\varpi_{1}\right)=$ $\mu$. By the minimality of $\mu$ we have $\mu=w_{0} w(\nu)$. Hence, all weights of the $G_{0}$-module $\mathfrak{g}_{1}$ are conjugate under $W$. As $\operatorname{dim} \mathfrak{g}_{1}^{\mu}=\operatorname{dim} \mathfrak{g}_{1}^{w \mu}$ for any $w \in W$, each weight occurs with multiplicity one. Now it is straightforward to see that $\mathfrak{g}_{1}=V$ is an irreducible standard $\mathfrak{g}_{0}^{(1)}$-module.

Suppose then that $\mathfrak{g}_{0}^{(1)}$ is of type $\mathrm{C}_{m}$ where $m \geq 1$, so that $G_{0}=\operatorname{Sp}(V)$ where $\operatorname{dim} V=2 m$. Then $\Gamma=-\varpi_{1}, \Lambda=3 \varpi_{1}$ and $\mathfrak{g}_{0} \subseteq \mathfrak{g}_{0}^{(1)} \oplus \mathbb{F}$, where $\mathbb{F}$ is a trivial $G_{0}$-module and $\mathfrak{g}_{0}^{(1)} \cong S^{2}(V)$ as $\mathfrak{g}_{0}^{(1)}$-modules (see (2.51)). As $\mathfrak{g}_{-1}$ is irreducible, the $\mathfrak{g}_{0}^{(1)}$-modules $\mathfrak{g}_{-1}$ and $S^{3}(V)$ are isomorphic (see Proposition 2.77 or Theorem 2.38). Identifying $\mathfrak{g}_{-1}$ with $S^{3}(V)$ and taking into account the fact that $S^{2}(V) \cong S^{2}(V)^{*}$, we can embed $\mathfrak{g}_{1}$ in the $\mathfrak{g}_{0}^{(1)}$-module

$$
\mathcal{N}:=S^{3}(V)^{*}+\left(S^{3}(V)^{*} \otimes S^{2}(V)^{*}\right),
$$

since

$$
\begin{aligned}
\mathfrak{g}_{1} \subseteq \operatorname{Hom}\left(\mathfrak{g}_{-1}, \mathfrak{g}_{0}\right) & \cong \mathfrak{g}_{-1}^{*} \otimes \mathfrak{g}_{0} \subseteq \mathfrak{g}_{-1}^{*} \otimes\left(\mathfrak{g}_{0}^{(1)} \oplus \mathbb{F}\right) \quad \text { where } \\
\mathfrak{g}_{-1}^{*} \otimes\left(\mathfrak{g}_{0}^{(1)} \oplus \mathbb{F}\right) & \cong S^{3}(V)^{*} \otimes\left(S^{2}(V)^{*} \oplus \mathbb{F}\right) \\
& \cong S^{3}(V)^{*}+\left(S^{3}(V)^{*} \otimes S^{2}(V)^{*}\right)=\mathcal{N}
\end{aligned}
$$

The group $G_{0}$ acts by automorphisms on the symmetric algebra $S(V)=\bigoplus_{j \geq 0} S^{j}(V)$, and the subspace $\mathcal{N} \subset S(V)^{*}$ is invariant under the dual action of $G_{0}$ on $S(V)^{*}$. The action of $\mathfrak{g}_{0}^{(1)}$ is the differential of this action of $G_{0}$. Now the multiplication in $S(V)$ induces a surjective $G_{0}$-module homomorphism $S^{2}(V) \otimes S^{3}(V) \rightarrow S^{5}(V)$. Hence, there is an embedding of $G_{0}$-modules

$$
\eta: S^{5}(V)^{*} \rightarrow S^{2}(V)^{*} \otimes S^{3}(V)^{*}
$$

Let

$$
\mathcal{N}_{0}:=\eta\left(S^{5}(V)^{*}\right)
$$

be the image and define

$$
R^{\prime}=\left\{ \pm \varepsilon_{i_{1}} \pm \varepsilon_{i_{2}} \pm \varepsilon_{i_{3}} \pm \varepsilon_{i_{4}} \pm \varepsilon_{i_{5}} \mid 1 \leq i_{1}, i_{2}, i_{3}, i_{4}, i_{5} \leq m\right\}
$$

Since $X(V)=\left\{ \pm \varepsilon_{1}, \ldots, \pm \varepsilon_{m}\right\}$, it is straightforward to see that $X(\mathcal{N}) \subseteq R^{\prime}$ and $\operatorname{dim} \mathcal{N}^{5 \varpi_{1}}=\operatorname{dim} \mathcal{N}_{0}^{5 \varpi_{1}}=1$. From this it follows that

$$
\begin{aligned}
& X_{+}\left(\mathcal{N} / \mathcal{N}_{0}\right) \subseteq\left\{3 \varpi_{1}+\varpi_{2}, 3 \varpi_{1}, 2 \varpi_{1}+\varpi_{3},\right. \\
&\left.\varpi_{1}+\varpi_{4}, \varpi_{1}+\varpi_{2}, \varpi_{5}, \varpi_{3}, \varpi_{1}\right\},
\end{aligned}
$$

where $\varpi_{5}$ is omitted if $m \leq 4, \varpi_{1}+\varpi_{4}$ is omitted if $m \leq 3,2 \varpi_{1}+\varpi_{3}$ and $\varpi_{3}$ are omitted if $m=1,2$, and $3 \varpi_{1}+\varpi_{2}, \varpi_{1}+\varpi_{2}$ are omitted if $m=1$. As a 
consequence, $X_{+}\left(\mathcal{N} / \mathcal{N}_{0}\right) \subset X_{1}(T)$, and any weight in $X_{+}\left(\mathcal{N} / \mathcal{N}_{0}\right)$ dominates $\varpi_{1}$.

We claim that $\mathfrak{g}_{1} \cap \mathcal{N}_{0}=0$. Indeed, suppose the contrary. Then Theorem 4.4 shows that the $\mathfrak{g}_{0}^{(1)}$-module $S^{5}(V)^{*} \cong \mathcal{N}_{0}$ contains a $\mathfrak{b}^{-}$-primitive vector $u$ of weight $\bar{\Gamma}=-\varpi_{1}$ or $-\bar{\Lambda}=-3 \varpi_{1}$. If $p>5$, then $S^{5}(V)$ is an irreducible $\mathfrak{g}_{0}^{(1)}$-module (see Lemma 2.79). Therefore, any $\mathfrak{b}^{-}$-primitive vector from $S^{5}(V)^{*}$ has weight $-5 \varpi_{1} \notin\left\{-\varpi_{1},-3 \varpi_{1}\right\}$, which is impossible.

Now assume $p=5$. By Lemma 2.79, there exists a trivial $\mathfrak{g}_{0}^{(1)}$-submodule $Y$ of $S^{5}(V)$, and the quotient $Y^{\prime}:=S^{5}(V) / Y$ has a $\mathfrak{b}^{+}$-primitive vector of weight $3 \varpi_{1}+\varpi_{2}$ if $m \geq 2\left(3 \varpi_{1}\right.$ if $\left.m=1\right)$. Set $Y^{\perp}:=\left\{\phi \in S^{5}(V)^{*} \mid\right.$ $\phi(Y)=0\}$. The $\mathfrak{g}_{0}^{(1)}$-modules $Y^{\perp}$ and $\left(Y^{\prime}\right)^{*}$ (resp. $S^{5}(V)^{*} / Y^{\perp}$ and $\left.Y^{*}\right)$ are isomorphic. If $m \geq 2$, then any $\mathfrak{b}^{-}$-primitive vector from $\left(Y^{\prime}\right)^{*}$ has weight $-3 \varpi_{1}-\varpi_{2} \notin\left\{-\varpi_{1},-3 \varpi_{1}\right\}$. But then $u \notin Y^{\perp}$, and so $S^{5}(V)^{*} / Y^{\perp} \cong Y^{*}$ contains a nonzero vector of weight $\bar{\Gamma}=-\varpi_{1}$ or $-\bar{\Lambda}=-3 \varpi_{1}$. As the $\mathfrak{g}_{0}^{(1)}$-module $Y^{*}$ is trivial, this is impossible. If $m=1$, this argument shows that $u$ has $\mathfrak{t}$-weight $-\bar{\Lambda}=-3 \varpi_{1} ; \quad Y^{\perp} \cong \mathfrak{U}\left(\mathfrak{n}^{+}\right) u ;$ and $\eta\left(\mathfrak{U}\left(\mathfrak{n}^{+}\right) u\right)$ is an irreducible submodule of the $\mathfrak{g}_{0}$-module $\mathfrak{g}_{1}$ (one should also take into account that $\left.\mathfrak{g}_{0}=\mathfrak{g}_{0}^{(1)} \oplus \mathfrak{Z}\left(\mathfrak{g}_{0}\right)\right)$. But then one can replace the triple $\left(f^{\Lambda}, \mathfrak{g}_{0}, e\right)$ by the triple $\left(f^{\Lambda}, \mathfrak{g}_{0}, \eta(u)\right)$ and argue as before, to conclude that that $\eta(u)$ has weight $\Gamma$. This is a contradiction, and it proves the claim.

Thus, the canonical epimorphism $\mathcal{N} \rightarrow \mathcal{N} / \mathcal{N}_{0}$ induces an injection $\mathfrak{g}_{-1} \rightarrow$ $\mathcal{N} / \mathcal{N}_{0}$. As $X_{+}\left(\mathcal{N} / \mathcal{N}_{0}\right) \subset X_{1}(T)$, Proposition 2.15 shows that the adjoint action of $\mathfrak{g}_{0}^{(1)}$ on $\mathfrak{g}_{-1}$ is induced by the differential of a rational action of $G_{0}$. The weights of the $G_{0}$-module $\mathfrak{g}_{1}$ belong to $X\left(\mathcal{N} / \mathcal{N}_{0}\right)$.

Again let $\mu$ be a minimal weight of the $G_{0}$-module $\mathfrak{g}_{1}$. Arguing as before we see that the weight space $\mathfrak{g}_{1}^{\mu}$ is $\mathfrak{t}$-stable and consists of $\mathfrak{b}^{-}$-primitive vectors relative to $\mathfrak{g}_{0}$. Let $e_{2}=e^{\Theta_{2}}$ be a nonzero weight vector for $\mathfrak{t}$ contained in $\mathfrak{g}_{1}^{\mu}$. By Theorem 4.4 (again, as in part (c) of the proof of Theorem 4.18), the image of $\mu$ in $X(T) / p X(T)$ coincides with $-3 \varpi_{1}$ or $-\varpi_{1}$. Since $p>3$, the description of $X_{+}\left(\mathcal{N} / \mathcal{N}_{0}\right)$ given above yields that either $w_{0} \mu=3 \varpi_{1}$ or $w_{0} \mu=\varpi_{1}$. Let $M^{\prime}$ denote the $G_{0}$-module generated by $e_{2}$. The orbit $N_{G_{0}}(T) \cdot e_{2}$ contains an eigenvector for $B^{+}=T N^{+}$of weight $w_{0} \mu$. Thus, $M^{\prime}$ is a homomorphic image of the Weyl module $V\left(w_{0} \mu\right)$; see Proposition $2.14(\mathrm{a})$.

Assume that $\mu=-3 \varpi_{1}$. Then $\Theta_{2}=-\Lambda$. Now $S^{3}(V)$ is an irreducible $\mathfrak{g}_{0}^{(1)}$-module generated by a $\mathfrak{b}^{+}$-primitive vector of weight $3 \varpi_{1}$. (This can be seen directly or by identifying $\mathfrak{g}_{0}^{(1)}$ with $H(2 m)_{0}$ and $S^{3}(V)$ with $\mathcal{O}(2 m)_{3}$, which has a $\mathfrak{b}^{+}$-primitive vector $x_{1}^{(3)}$, and then by appealing to $[\mathbf{S t}$, Lem. 5.2.2] or Proposition 2.77(a)). By Weyl's dimension formula (2.11), $\operatorname{dim} V\left(3 \varpi_{1}\right)=\operatorname{dim} S^{3}(V)$. In conjunction with Proposition 2.13 this implies that $S^{3}(V) \cong L\left(3 \varpi_{1}\right)$ as $\mathfrak{g}_{0}^{(1)}$-modules. But then $V\left(3 \varpi_{1}\right) \cong L\left(3 \varpi_{1}\right) \cong M^{\prime}$ as $G_{0}$-modules; see Proposition 2.14(a). Hence, $M^{\prime}$ is an irreducible $\mathfrak{g}_{0}$-module, 
and we can, as before, replace the triple $\left(f^{\Lambda}, \mathfrak{g}_{0}, e\right)$ by the triple $\left(f^{\Lambda}, \mathfrak{g}_{0}, e_{2}\right)$ to deduce that $\Theta_{2} \neq-\Lambda$. This contradiction shows that $-\varpi_{1}$ is the only minimal weight of the $G_{0}$-module $\mathfrak{g}_{1}$.

Since $\mathfrak{g}$ is irreducible and transitive, it must be that $\operatorname{dim} \mathfrak{g}_{1}^{\mu}=1$. We have already established that $\nu \geq \varpi_{1}$ for any $\nu \in X_{+}\left(\mathfrak{g}_{1}\right)$. From this it is immediate that all weights of the $G_{0}$-module $\mathfrak{g}_{1}$ are conjugate under the Weyl group of $G_{0}$. Using these properties and reasoning as above, we see that $\mathfrak{g}_{1}=V$. We have considered all the cases, so the lemma is proved.

\subsection{Determining the negative part when $\mathfrak{g}_{1}$ is irreducible}

In Lemma 4.30 below, we show that if the Lie algebra $\widehat{\mathfrak{g}}$ generated by the local part of $\mathfrak{g}$ is isomorphic to one of the graded Lie algebras of Cartan type $S, C S, H$, or $C H$ (so that $\mathfrak{g}_{1}$ is an irreducible $\mathfrak{g}_{0}$-module), then $\mathfrak{g}$ itself must be depth-one. Except for a couple of special cases which must be treated separately, we are able to verify the lemma by applying previous results to the Lie algebra generated by $\mathfrak{g}_{0}$, a homogeneous space $\widehat{\mathfrak{g}}_{k}$ of $\widehat{\mathfrak{g}}$ for $k \geq 1$, and a certain $\mathfrak{g}_{0}$-submodule $V_{-k}$ of $\mathfrak{g}_{-k}$.

LEMma 4.30. Let $\mathfrak{g}$ be a finite-dimensional graded Lie algebra satisfying conditions (1)-(5). Suppose that the subalgebra $\widehat{\mathfrak{g}}$ generated by the local part $\mathfrak{g}_{-1} \oplus \mathfrak{g}_{0} \oplus \mathfrak{g}_{1}$ is isomorphic one of the restricted Lie algebras of Cartan type $S(m+1 ; \underline{1})^{(1)}, m \geq 2, S(m+1 ; \underline{1})^{(1)} \oplus \mathbb{F} \mathfrak{D}_{1}, m \geq 2, H(2 m ; \underline{1})^{(2)}, m \geq 1$, or $H(2 m ; \underline{1})^{(2)} \oplus \mathbb{F} \mathfrak{D}_{1}, m \geq 1$, with its natural grading. Then $\mathfrak{g}_{-2}=0$.

Proof. (a) Set $\widehat{\mathfrak{g}}_{j}=\widehat{\mathfrak{g}} \cap \mathfrak{g}_{j}$ for $-q \leq j \leq r$. By our hypothesis on $\widehat{\mathfrak{g}}$, the commutator $\mathfrak{g}_{0}^{(1)}$ is a classical simple Lie algebra of type $\mathrm{A}_{m}$ or $\mathrm{C}_{m}$. Let $\mathfrak{t}$ denote the toral subalgebra of $\mathfrak{g}_{0}$, and let $f^{\Lambda}$ be a $\mathfrak{b}^{+}$-primitive vector of weight $\Lambda$ from $\mathfrak{g}_{-1}$. In the $\mathrm{A}_{m}$-case, we assume as before that $\Lambda=\varpi_{m}$. In this case, we have by Lemma 2.78 (a) that when $m \geq 2$, the $\mathfrak{g}_{0}^{(1)}$-module $\widehat{\mathfrak{g}}_{k}$ is generated by a $\mathfrak{b}^{-}$-primitive vector $e^{-\varpi_{1}-(k+1) \varpi_{m}}, k=2,3$. If $\Phi$ is of type $\mathrm{C}_{m}, m \geq 1$, and $p>5$, the same lemma shows that $\widehat{\mathfrak{g}}_{k}$ is generated by a $\mathfrak{b}^{-}$-primitive vector $e^{-(k+2) \varpi_{1}}, k=2,3$. If $p=5$ and $\Phi$ is of type $\mathrm{C}_{m}, m \geq 2$, then $\widehat{\mathfrak{g}}_{2}$ is generated by a $\mathfrak{b}^{-}$-primitive vector $e^{-4 \varpi_{1}}$ and $\widehat{\mathfrak{g}}_{3}$ is generated by a $\mathfrak{b}^{-}$-primitive vector $e^{-3 \varpi_{1}-\varpi_{2}}$; see Lemma 2.78 (b). Finally, in the $\mathrm{A}_{1}, p=5$ case, $\widehat{\mathfrak{g}}_{2}$ is generated by a $\mathfrak{b}^{-}$-primitive vector $e^{-4 \varpi_{1}}$, and $\widehat{\mathfrak{g}}_{3}$ is generated by a $\mathfrak{b}^{-}$-primitive vector $e^{-3 \varpi_{1}}$, again by Lemma $2.78(\mathrm{~b})$.

(b) By 1-transitivity (1.3), the $\mathfrak{g}_{0}$-submodule $\mathfrak{g}_{-i}$ is isomorphic to a submodule of $\operatorname{Hom}\left(\mathfrak{g}_{1}, \mathfrak{g}_{-(i-1)}\right)(i>0)$. This can be used to show that each $\mathfrak{g}_{-i}$ with $i>0$ is a restricted $\mathfrak{g}_{0}^{(1)}$-module. Indeed for $i=1$, this is Remark 4.6. By transitivity (1.2), $\mathfrak{g}_{1}$ is isomorphic to a submodule of $\operatorname{Hom}\left(\mathfrak{g}_{-1}, \mathfrak{g}_{0}\right)$; hence it is a restricted $\mathfrak{g}_{0}^{(1)}$-module. (Note that $\mathfrak{g}_{0}$ is a restricted $\mathfrak{g}_{0}^{(1)}$-module too, as the restriction map on $\mathfrak{g}_{0}^{(1)}$ is induced by that on $\mathfrak{g}_{0}$.) By our induction 
assumption, the $\mathfrak{g}_{0}^{(1)}$-module $\mathfrak{g}_{-(i-1)}$ is restricted. But then so is the $\mathfrak{g}_{0}^{(1)}$ module $\operatorname{Hom}\left(\mathfrak{g}_{1}, \mathfrak{g}_{-(i-1)}\right)$ along with its submodule $\mathfrak{g}_{-i}$. This completes the induction step.

(c) Suppose that $\Phi \neq \mathrm{A}_{1}$ if $p=5$. We will show that $\left[\mathfrak{g}_{-k}, \widehat{\mathfrak{g}}_{k}\right]=0$ for $k=2,3$. Suppose the contrary, and let $\mathfrak{a}_{-k}:=\left\{x \in \mathfrak{g}_{-k} \mid\left[x, \widehat{\mathfrak{g}}_{k}\right]=0\right\}$ for $k=2,3$. Let $V_{-k}$ denote a $\mathfrak{g}_{0}$-submodule of $\mathfrak{g}_{-k}$ containing $\mathfrak{a}_{-k}$ such that $U_{-k}:=V_{-k} / \mathfrak{a}_{-k}$ is irreducible. Both $V_{-k}$ and $\mathfrak{a}_{-k}$ are $\mathfrak{g}_{0}^{(1)}$-submodules of $\mathfrak{g}_{-k}$, and hence are restricted over $\mathfrak{g}_{0}^{(1)}$, by (b). Therefore, so is the quotient module $U_{-k}:=V_{-k} / \mathfrak{a}_{-k}$.

(c1) By Engel's theorem, the subalgebra $\mathfrak{n}^{+}$of $\mathfrak{g}_{0}^{(1)}$ annihilates a nonzero vector on any $\mathfrak{g}_{0}$-module $E$ endowed with a $p$-character which vanishes on $\mathfrak{g}_{0}^{(1)}$. This means that $E_{0}=\left\{v \in E \mid e_{\alpha} \cdot v=0\right.$ for all $\left.\alpha \in \Phi^{+}\right\}$is a nonzero $\mathfrak{t}$-module. But $\mathfrak{t}$ is diagonalizable for any $\mathfrak{g}_{0}$-module affording a $p$-character. So there is $\lambda \in \mathfrak{t}^{*}$ such that $E_{0}^{\lambda}=\left\{v \in E_{0} \mid t . v=\lambda(t) v\right.$ for all $\left.t \in \mathfrak{t}\right\}$ is nonzero.

(c2) Let $S^{\{k\}}=\bigoplus_{i \in k \mathbb{Z}} S_{i}^{\{k\}}$ be the graded subalgebra of $\mathfrak{g}$ generated by $V_{-k}, \mathfrak{g}_{0}$, and $\widehat{\mathfrak{g}}_{k}$, and let $\mathcal{M}\{k k\}$ be the Weisfeiler radical $\mathcal{M}\left(S^{\{k\}}\right)$ of $S^{\{k\}}$. Obviously, $\mathcal{M}^{\{k\}} \cap S_{-k}^{\{k\}}=\mathfrak{a}_{-k}$, and $S_{k}^{\{k\}}=\mathfrak{g}_{k}$. Let $\bar{S}^{\{k\}}:=S^{\{k\}} / \mathcal{M}^{\{k\}}$. Then $\bar{S}_{-k}^{\{k\}} \cong U_{-k}$ as $\mathfrak{g}_{0}$-modules, and $\bar{S}_{k}^{\{k\}} \cong \mathfrak{g}_{k}$. Part (c1) shows that we can find a $\mathfrak{b}^{+}$-primitive vector in $U_{-k}$, say $f^{\mu_{k}}$. Because $\left[\bar{S}_{-k}^{\{k\}}, \bar{S}_{k}^{\{k\}}\right] \neq 0$, we must have $\left[f^{\mu_{k}}, e^{\lambda_{k}}\right] \neq 0$ where $e^{\lambda_{k}}$ is a $\mathfrak{b}^{-}$-primitive vector of $\mathfrak{g}_{k}$ generating $\mathfrak{g}_{k}$ as a $\mathfrak{g}_{0}^{(1)}$-module.

(c3) If $\mu_{k} \neq-\lambda_{k}$ and $\mu_{k}\left(\mathfrak{t} \cap \mathfrak{g}_{0}^{(1)}\right) \neq 0$, then the graded Lie algebra $\bar{S}^{\{k\}}$ satisfies all the conditions of Theorem 4.4. But then $-\lambda_{k} \in\left\{\varpi_{1}, \varpi_{m}, 2 \varpi_{1}+\right.$ $\left.\varpi_{m}, \varpi_{1}+2 \varpi_{m}\right\}$ if $\Phi \cong \mathrm{A}_{m}, m \geq 2$, and $-\lambda_{k} \in\left\{\varpi_{1}, 3 \varpi_{1}\right\}$ if $\Phi \cong \mathrm{C}_{m}, m \geq 1$. Using our description of the $\lambda_{k}$ 's in part (a), it is easy to check that this is not the case. Hence, either $\mu_{k}\left(\mathfrak{t} \cap \mathfrak{g}_{0}^{(1)}\right)=0$, or $\mu_{k}=-\lambda_{k}$.

If $\mu_{k}\left(\mathfrak{t} \cap \mathfrak{g}_{0}^{(1)}\right)=0$, then it follows from the classification of the irreducible restricted representations of a classical Lie algebra that $U_{-k}$ is a trivial $\mathfrak{g}_{0}^{(1)}$ module. But then ad $f^{\mu_{k}}$ induces a nonzero $\mathfrak{g}_{0}^{(1)}$-module homomorphism $\phi_{k}: \mathfrak{g}_{k} \rightarrow \mathfrak{g}_{0}$. Since $\mathfrak{g}_{k}$ is generated by $e^{\lambda_{k}}$ as a $\mathfrak{g}_{0}^{(1)}$-module, it must be that $\phi_{k}\left(e^{\lambda_{k}}\right)$ is a $\mathfrak{b}^{-}$-primitive vector in $\mathfrak{g}_{0}$. But $-\lambda_{k}$ is neither zero nor the highest root of $\Phi$; see part (a) and our assumption in part (c). Hence, $\mu_{k}\left(\mathfrak{t} \cap \mathfrak{g}_{0}^{(1)}\right) \neq 0$.

(c4) Now suppose $\mu_{k}=-\lambda_{k}$. We first assume that $\mathfrak{g}_{k}$ is an irreducible $\mathfrak{g}_{0}^{(1)}$ module and let $\overline{\mathcal{N}}^{\{k\}}$ denote the maximal graded ideal of $\bar{S}^{\{k\}}$ contained in $\bigoplus_{i \geq 1} \bar{S}_{i}^{\{k\}}$. Theorem 3.22 shows that $\bar{S}^{\{k\}} / \overline{\mathcal{N}}^{\{k\}}$ is a classical Lie algebra with one of its standard gradings (note that $\bar{S}^{\{k\}} / \overline{\mathcal{N}}^{\{k\}}$ cannot be a Melikyan 
algebra in view of our assumption in part (c)). As in the proof of Lemma 4.28 , one then observes that the the $\mathfrak{g}_{0}^{(1)}$-module $\bar{S}_{-k}^{\{k\}} \cong U_{-k}$ must have a $\mathfrak{b}^{+}$-primitive vector whose weight is in the list

$$
\begin{array}{lr}
\left\{2 \varpi_{1}, \varpi_{1}, \varpi_{2}, \varpi_{m-1}, \varpi_{m}, 2 \varpi_{m}\right\}, & m \geq 8, \\
\left\{2 \varpi_{1}, \varpi_{1}, \varpi_{2}, \varpi_{3}, \varpi_{5}, \varpi_{6}, \varpi_{7}, 2 \varpi_{7}\right\}, & m=7, \\
\left\{\varpi_{i} \mid 1 \leq i \leq m\right\} \cup\left\{2 \varpi_{1}, 2 \varpi_{m}\right\}, & 2 \leq m \leq 6,
\end{array}
$$

for $\mathfrak{g}_{0}^{(1)}$ of type $\mathrm{A}_{m}$, and in the list

$$
\begin{array}{lrl}
\left\{\varpi_{1}\right\}, & m \geq 4, \\
\left\{\varpi_{1}, \varpi_{m}\right\}, & m=2,3, \\
\left\{\varpi_{1}, 2 \varpi_{1}, 3 \varpi_{1}\right\}, & m=1,
\end{array}
$$

for $\mathfrak{g}_{0}^{(1)}$ of type $\mathrm{C}_{m}$. On the other hand, it follows from our discussion in part (a) that $\mu_{k}=\varpi_{1}+(k+1) \varpi_{m}, k=2,3$, if $\mathfrak{g}_{0}^{(1)}$ is of type $\mathrm{A}_{m}, m \geq 2$, and $\mu_{k}=(k+2) \varpi_{1}, k=2,3$, for $p>5$ and $\mu_{k} \in\left\{4 \varpi_{1}, 3 \varpi_{1}+\varpi_{2}\right\}$ for $p=5$ and $m \geq 2$, if $\mathfrak{g}_{0}^{(1)}$ is of type $\mathrm{C}_{m}, m \geq 1$. These linear functions do not appear on the list. Therefore, the case we are considering cannot occur.

(c5) Next assume that $\mu_{k}=-\lambda_{k}$ and $\mathfrak{g}_{k}$ is a reducible $\mathfrak{g}_{0}^{(1)}$ module. Proposition 2.77 (b) together with Theorem 2.69 (i) show that $m+k \equiv 0 \bmod p$ and $\widehat{\mathfrak{g}}$ is isomorphic to one of $S(m+1 ; \underline{1})^{(1)}$ or $S(m+1 ; \underline{1})^{(1)} \oplus \mathbb{F} \mathfrak{D}_{1}$. Theorem 2.69 then says that $\mathfrak{g}_{k} \cong \bar{S}_{k}^{\{k\}}$ contains an irreducible $\mathfrak{g}_{0}^{(1)}$-submodule $\mathfrak{g}_{k}^{\sharp}$ with a $\mathfrak{b}^{+}$-primitive vector of weight $k \varpi_{1}$ such that the quotient module $\mathfrak{g}_{k} / \mathfrak{g}_{k}^{\sharp}$ is irreducible. Let $e^{-k \varpi_{m}}$ be a $\mathfrak{b}^{-}$-primitive vector of the $\mathfrak{g}_{0}^{(1)}$-module $\mathfrak{g}_{k}^{\sharp} \subset \bar{S}_{k}^{\{k\}}$. Note that $\left[f^{\mu_{k}}, e^{k \varpi_{m}}\right]$ is a multiple of a highest root vector in $\bar{S}_{0}^{\{k\}} \cong \mathfrak{g}_{0}$. Since $k \in\{2,3\}$, Theorem 4.4 implies $\left[f^{\mu_{k}}, e^{k \varpi_{m}}\right]=0$. But then $\left[f^{\mu_{k}}, \mathfrak{g}_{k}^{\sharp}\right]=0$, forcing $\left[\bar{S}_{k}^{\{k\}}, \mathfrak{g}_{k}^{\sharp}\right]=0$.

As in part (c4), we let $\overline{\mathcal{N}}^{\{k\}}$ denote the maximal graded ideal of $\bar{S}^{\{k\}}$ contained in $\bigoplus_{i \geq 1} \bar{S}_{i}^{\{k\}}$. The above discussion then shows that $\overline{\mathcal{N}}_{k}^{\{k\}}=\mathfrak{g}_{k}^{\sharp}$ and

$$
\bar{S}_{k}^{\{k\}} / \overline{\mathcal{N}}_{k}^{\{k\}} \cong \mathfrak{g}_{k} / \mathfrak{g}_{k}^{\sharp} \cong U_{-k}^{*}=\left(\bar{S}_{-k}^{\{k\}}\right)^{*}
$$

as $\mathfrak{g}_{0}^{(1)}$-modules. But then Theorem 3.22 applies to the graded Lie algebra $\bar{S}^{\{k\}} / \overline{\mathcal{N}}^{\{k\}}$. Repeating the argument from part (c4) we now deduce that the present case cannot occur. This shows that under the main assumption of part (c) we have $\left[\mathfrak{g}_{k}, \widehat{\mathfrak{g}}_{k}\right]=0$ for $k=2,3$.

(c6) As $\widehat{\mathfrak{g}}_{2} \neq 0$, it follows that $\left[\mathfrak{g}_{-1},\left[\mathfrak{g}_{-1}, \widehat{\mathfrak{g}}_{2}\right] \neq 0\right.$ by transitivity. As $\left[\mathfrak{g}_{-2}, \widehat{\mathfrak{g}}_{2}\right]=0$, it follows that $\left[\mathfrak{g}_{-2},\left[\mathfrak{g}_{-1},\left[\mathfrak{g}_{1}, \widehat{\mathfrak{g}}_{2}\right]\right]\right]=0$. By assumption, $\mathfrak{g}_{-2} \neq 0$, so that $\left[\mathfrak{g}_{-2}, \mathfrak{g}_{1}\right]=\mathfrak{g}_{-1}$, by irreducibility and 1-transitivity. But 
then

$$
\begin{aligned}
{\left[\mathfrak{g}_{-3}, \widehat{\mathfrak{g}}_{3}\right] } & \supseteq\left[\left[\mathfrak{g}_{-1}, \mathfrak{g}_{-2}\right],\left[\mathfrak{g}_{1}, \widehat{\mathfrak{g}}_{2}\right]\right]=\left[\mathfrak{g}_{-1},\left[\mathfrak{g}_{-2},\left[\mathfrak{g}_{1}, \widehat{\mathfrak{g}}_{2}\right]\right]\right] \\
& =\left[\mathfrak{g}_{-1},\left[\left[\mathfrak{g}_{-2}, \mathfrak{g}_{1}\right], \widehat{\mathfrak{g}}_{2}\right]\right]=\left[\mathfrak{g}_{-1},\left[\mathfrak{g}_{-1}, \widehat{\mathfrak{g}}_{2}\right]\right] \neq 0 .
\end{aligned}
$$

This contradiction shows that $\mathfrak{g}_{-2}=0$ if $p>5$, or if $p=5$ and $\Phi$ is not of type $A_{1}$.

(d) Now suppose that $p=5$ and $\Phi$ is of type $A_{1}$. By our initial assumption, the graded Lie algebra $\widehat{\mathfrak{g}}$ is then isomorphic to either $H(2 ; \underline{1})^{(2)}$ or $H(2 ; \underline{1})^{(2)} \oplus$ $\mathbb{F} \mathfrak{D}_{1}$. By Proposition 2.77(a) and Lemma 2.78(b), the $\mathfrak{g}_{0}^{(1)}$-module $\mathfrak{g}_{2}$ is irreducible and generated by a $\mathfrak{b}^{-}$-primitive vector $e^{-4 \varpi_{1}}$. If we reason as above, it is not difficult to observe that $\left[\mathfrak{g}_{-2}, \widehat{\mathfrak{g}}_{2}\right]=0$. This forces $\left[\mathfrak{g}_{-3}, \widehat{\mathfrak{g}}_{3}\right] \neq$ 0 ; see the above calculation. Since $\widehat{\mathfrak{g}}$ is generated by its local part, the commutator subalgebra $\widehat{\mathfrak{g}}^{(1)}$ is simple. Moreover, $\widehat{\mathfrak{g}}_{i} \subset \widehat{\mathfrak{g}}^{(1)}$ for any $i \neq 0$ and $\widehat{\mathfrak{g}}^{(1)} \cap \mathfrak{g}_{2(p-1)-2}=0$; see Lemma 2.80. As $p=5$, this gives $\widehat{\mathfrak{g}}_{6}=0$. By Lemma 2.80 again, $\widehat{\mathfrak{g}}_{3}$ is an irreducible $\mathfrak{g}_{0}^{(1)}$-module generated by a $\mathfrak{b}^{-}$primitive vector $e^{-3 \varpi_{1}}$. Consequently, $\left[\mathfrak{g}_{-3}, e^{-3 \varpi_{1}}\right] \neq 0$.

As before, let $V_{-3}$ be a $\mathfrak{g}_{0}$-submodule of $\mathfrak{g}_{-3}$ containing $\mathfrak{a}_{-3}:=\{x \in$ $\left.\mathfrak{g}_{-3} \mid\left[x, \widehat{\mathfrak{g}}_{3}\right]=0\right\}$ and such that the quotient module $U_{-3}=V_{-3} / \mathfrak{a}_{-3}$ is irreducible. Let $S^{\{3\}}$ be the graded subalgebra generated by $V_{-3}, \mathfrak{g}_{0}$, and $\widehat{\mathfrak{g}}_{3}$. Let $\mathcal{M}^{\{3\}}$ denote the maximal ideal of $S^{\{3\}}$ contained in the negative part $\bigoplus_{i<0} S_{i}^{\{3\}}$. Set $\bar{S}^{\{3\}}:=S^{\{3\}} / \mathcal{M}\{3\}$. Clearly, $\bar{S}^{\{3\}}$ is graded:

$$
\bar{S}^{\{3\}}=\bigoplus_{i \in 3 \mathbb{Z}}\left(\bar{S}^{\{3\}}\right)_{i}
$$

and $\bar{S}_{-3}^{\{3\}} \cong U_{-3}, \bar{S}_{0}^{\{3\}} \cong \mathfrak{g}_{0}$, and $S_{3}^{\{3\}} \cong \overline{\mathfrak{g}}_{3}$. By similar reasoning, we find a $\mathfrak{b}^{+}$-primitive vector $f^{\mu_{3}} \in U_{-3}$ and show that $\left[f^{\mu_{3}}, \overline{e^{-3 \varpi_{1}}}\right] \neq 0$; here $\overline{e^{-3 \varpi 1}}$ denotes the image of $e^{-3 \varpi_{1}}$ in $\bar{S}^{\{3\}}$.

If $\mu_{3}\left(\mathfrak{t} \cap \mathfrak{g}_{0}^{(1)}\right)=0$, then, as above, $U_{-3}=\mathbb{F} f^{\mu_{3}}$, and ad $f^{\mu_{3}}$ induces a nonzero $\mathfrak{g}_{0}^{(1)}$-module homomorphism from $\widehat{\mathfrak{g}}_{3}$ into $\mathfrak{g}_{0}$. Since this situation is easily seen to be impossible, we have $\mu_{3}\left(\mathfrak{t} \cap \mathfrak{g}_{0}^{(1)}\right) \neq 0$. If $\mu_{3} \neq 3 \varpi_{1}$, Theorem 4.4 applies to yield that $\left[f^{\mu_{3}}, \overline{e^{-3 \varpi_{1}}}\right]=f_{1}$, a $\mathfrak{b}^{-}$-primitive vector of $\mathfrak{g}_{0}^{(1)} \cong \mathfrak{s l}_{2}$. Hence, $\mu_{3}=\varpi_{1}$. Let $f^{3 \varpi_{1}}$ be a $\mathfrak{b}^{+}$-primitive vector of $\widehat{\mathfrak{g}}_{3}$, and let $\overline{f^{3 \varpi_{1}}}$ denote its image in $\bar{S}^{\{3\}}$. Because $\left[f^{\mu_{3}}, \overline{f^{3 \varpi_{1}}}\right] \in \mathfrak{g}_{0}^{4 \varpi_{1}}$ we have $\left[f^{\mu_{3}}, \overline{f^{3 \varpi_{1}}}\right]=0$. Therefore,

$$
\left[f^{\mu_{3}},\left[\overline{f^{3 \varpi_{1}}}, \overline{e^{-3 \varpi_{1}}}\right]\right]=-\left[f_{1}, \overline{f^{3 \varpi_{1}}}\right] \neq 0,
$$

forcing $\left(\bar{S}^{\{3\}}\right)_{6} \neq 0$. Since this contradicts $\widehat{\mathfrak{g}}_{6}=0$, we conclude that $\mu_{3}=$ $3 \varpi_{1}$.

Let $\mathcal{J}$ be the maximal graded ideal of $\bar{S}^{\{3\}}$ contained in the subspace $\bigoplus_{|i|>3} \bar{S}_{i}^{\{3\}}$. By construction, the graded Lie algebra $\bar{S}^{\{3\}} / \mathcal{J}$ satisfies all the conditions of Theorem 3.22. Since $\bar{S}^{\{3\}} / \mathcal{J}$ is generated by its local part, it 
cannot be isomorphic to a Melykian algebra. Hence, $\bar{S}^{\{3\}} / \mathcal{J}$ is isomorphic to a graded Lie algebra of type $\mathrm{G}_{2}$ associated with the Dynkin diagram

$$
\stackrel{\circ}{\alpha_{1}-\mu_{3}}
$$

(see Theorem 3.22 and [Bou1]). But then $\left(\bar{S}^{\{3\}}\right)_{6} \neq\left(\left(\bar{S}^{\{3\}}\right)_{6} \cap \mathcal{J}\right)$ to force $\widehat{\mathfrak{g}}_{6} \neq 0$. This contradiction shows that $\mathfrak{g}_{-2}$ must be zero, as desired.

\subsection{Determining the negative part when $\mathfrak{g}_{1}$ is reducible}

Here we show that if the local Lie algebra $\widehat{\mathfrak{g}}$ is a restricted Lie algebra of Cartan type $W$ or $K$, then the negative part of $\mathfrak{g}$ must coincide with the negative part of $\widehat{\mathfrak{g}}$. Under these hypotheses, $\mathfrak{g}_{1}$ is the direct sum of two irreducible $\mathfrak{g}_{0}$-modules in most cases, so we can look at the Lie algebras generated by $\mathfrak{g}_{-1}, \mathfrak{g}_{0}$, and various submodules of $\mathfrak{g}_{1}$ and apply previous results to (quotients of) them.

LEMma 4.33. Let $\mathfrak{g}=\bigoplus_{i=-q}^{r} \mathfrak{g}_{i}$ be a finite-dimensional graded Lie algebra which satisfies conditions (1)-(5). Suppose that the subalgebra $\widehat{\mathfrak{g}}$ generated by the local part $\mathfrak{g}_{-1} \oplus \mathfrak{g}_{0} \oplus \mathfrak{g}_{1}$ is isomorphic to a graded Lie algebra of Cartan type $W(m ; \underline{1}), m \geq 2$, or $K(2 m+1 ; \underline{1})^{(1)}$. Then $\mathfrak{g}_{i}=\widehat{\mathfrak{g}}_{i}$ for $i<0$.

Proof. (a) We first assume that $\widehat{\mathfrak{g}} \neq W(m ; \underline{1})$ if $m+1 \equiv 0 \bmod p$. In this case the $\mathfrak{g}_{0}$-module $\mathfrak{g}_{1}$ decomposes into the direct sum of two $\mathfrak{g}_{0}$-submodules $\mathfrak{g}_{1}^{\sharp}$ and $\mathfrak{g}_{1}^{\dagger}$. For $\widehat{\mathfrak{g}} \cong W(m ; \underline{1})$ this follows from Theorem 2.69. For $\widehat{\mathfrak{g}} \cong$ $K(2 m+1, \underline{1})^{(1)}$ we let $\mathfrak{g}_{1}^{\dagger}$ denote the span of all $D_{K}\left(x_{i}^{(1)} x_{j}^{(1)} x_{k}^{(1)}\right)$ with $1 \leq$ $i, j, k \leq 2 m$, and we put $\mathfrak{g}_{1}^{\sharp}:=\left\{D_{K}\left(x_{i}^{(1)} x_{2 m+1}^{(1)}\right) \mid 1 \leq i \leq 2 m\right\}$. Recall that here we have $\mathfrak{g}_{0}^{(1)} \cong \mathfrak{s p}(V)$ where $V$ is the span of $x_{i}^{(1)}$ with $1 \leq i \leq 2 m$. Using Proposition 2.61 it is straightforward to see that $\mathfrak{g}_{1}^{\dagger} \cong S^{3}(V)$ and $\mathfrak{g}_{1}^{\sharp} \cong V \cong \mathfrak{g}_{-1}$ as $\mathfrak{g}_{0}^{(1)}$-modules.

Let $\widehat{\mathfrak{g}}^{\dagger}$ and $\widehat{\mathfrak{g}}^{\sharp}$ denote the graded subalgebras of $\mathfrak{g}$ generated by $\mathfrak{g}_{-1} \oplus \mathfrak{g}_{0} \oplus$ $\mathfrak{g}_{1}^{\dagger}$ and $\mathfrak{g}_{-1} \oplus \mathfrak{g}_{0} \oplus \mathfrak{g}_{1}^{\sharp}$, respectively. It follows from our general assumption and Proposition 2.61(ii) that $\widehat{\mathfrak{g}}^{\sharp}$ is 1-transitive and $\widehat{\mathfrak{g}}_{-2}=\mathfrak{Z}\left(\widehat{\mathfrak{g}}^{\dagger}\right)$. Applying Theorem 2.66 we now deduce that the graded Lie algebra $\widehat{\mathfrak{g}}^{\dagger} / \widehat{\mathfrak{g}}_{-2}$ is isomorphic to one of Cartan type Lie algebras $S(m ; \underline{1})^{(1)} \oplus \mathbb{F} \mathfrak{D}_{1}, m \geq 3$, or $H(2 m ; \underline{1})^{(2)} \oplus \mathbb{F} \mathfrak{D}_{1}, m \geq 1$, while $\widehat{\mathfrak{g}}^{\sharp}$ is isomorphic to a classical Lie algebra of type $\mathrm{A}_{m}$ or $\mathrm{C}_{m+1}$.

(b) Let us denote by $\mathfrak{g}^{\sharp}$ the graded subalgebra of $\mathfrak{g}$ generated by the subspaces $\mathfrak{g}_{1}^{\sharp}$ and $\mathfrak{g}_{i}$ with $i \leq 0$. Let $\mathcal{M}\left(\mathfrak{g}^{\sharp}\right)$ be the maximal ideal of $\mathfrak{g}^{\sharp}$ contained in the negative part $\bigoplus_{i<0} \mathfrak{g}_{i}$ of $\mathfrak{g}^{\sharp}$. Clearly, $\mathcal{M}\left(\mathfrak{g}^{\sharp}\right) \subseteq \bigoplus_{i<-2} \mathfrak{g}_{i}$. Since $\mathfrak{g}_{1}^{\sharp} \cong \mathfrak{g}_{-1}^{*}$ as $\mathfrak{g}_{0}$-modules, the quotient algebra $\overline{\mathfrak{g}}^{\sharp}:=\mathfrak{g}^{\sharp} / \mathcal{M}\left(\mathfrak{g}^{\sharp}\right)$ satisfies all the conditions of Theorem 3.22. Our general assumption implies 
$\left[\left[\mathfrak{g}_{-1}, \mathfrak{g}_{-1}\right], \mathfrak{g}_{-1}\right]=0$. Since the subalgebra generated by the local part of a Melikyan algebra is isomorphic to a Lie algebra of type $\mathrm{G}_{2}$ graded according to the long simple root, Theorem 3.22 yields that $\overline{\mathfrak{g}}^{\sharp}$ is classical. As a corollary, if $\widehat{\mathfrak{g}} \cong W(m ; \underline{1})$, then $\mathfrak{g}_{-2} \subseteq \mathcal{M}\left(\mathfrak{g}^{\sharp}\right)$, and if $\widehat{\mathfrak{g}} \cong K(2 m+1 ; \underline{1})^{(1)}$, then $\mathfrak{g}_{-3} \subseteq \mathcal{M}\left(\mathfrak{g}^{\sharp}\right)$ and $\mathcal{M}\left(\mathfrak{g}^{\sharp}\right)_{-2}$ has codimension 1 in $\mathfrak{g}_{-2}$.

Clearly, $\mathcal{M}\left(\mathfrak{g}^{\sharp}\right)_{-2}=\left\{x \in \mathfrak{g}_{-2} \mid\left[x, \mathfrak{g}_{1}^{\sharp}\right]=0\right\}$. It follows easily from this and our general assumption that $\widehat{\mathfrak{g}}_{-2} \cap \mathcal{M}\left(\mathfrak{g}^{\sharp}\right)_{-2}=0$ and that $\widehat{\mathfrak{g}}_{-2} \neq 0$ only if $\widehat{\mathfrak{g}} \cong K(2 m+1 ; \underline{1})^{(1)}$. Therefore,

$$
\mathfrak{g}_{-2}=\widehat{\mathfrak{g}}_{-2} \oplus \mathcal{M}\left(\mathfrak{g}^{\sharp}\right)_{-2} .
$$

(c) Let $\mathfrak{g}^{\dagger}$ denote the subalgebra of $\mathfrak{g}$ generated by the subspaces $\mathfrak{g}_{1}^{\dagger}$ and $\mathfrak{g}_{i}, i \leq 0$. Let $\mathcal{M}\left(\mathfrak{g}^{\dagger}\right)$ be the maximal ideal of $\mathfrak{g}^{\dagger}$ contained in the negative part $\bigoplus_{i<0} \mathfrak{g}_{i}$ of $\mathfrak{g}^{\dagger}$. Our discussion in part (a) shows that $\widehat{\mathfrak{g}}_{-2} \subseteq \mathcal{M}\left(\mathfrak{g}^{\dagger}\right)$ and the quotient algebra $\overline{\mathfrak{g}^{\dagger}}:=\mathfrak{g}^{\dagger} / \mathcal{M}\left(\mathfrak{g}^{\dagger}\right)$ satisfies all of the conditions of Lemma 4.30. Applying this lemma, we obtain that $\mathcal{M}\left(\mathfrak{g}^{\dagger}\right)_{-2}=\mathfrak{g}_{-2}$. As $\mathcal{M}\left(\mathfrak{g}^{\sharp}\right)_{-2}=\left\{x \in \mathfrak{g}_{-2} \mid\left[x, \mathfrak{g}_{1}^{\sharp}\right]=0\right\}$, we have $\left[\mathcal{M}\left(\mathfrak{g}^{\dagger}\right)_{-2}, \mathfrak{g}_{1}^{\sharp}+\mathfrak{g}_{1}^{\dagger}\right]=0$. Since $\mathfrak{g}$ is 1 -transitive and $\mathfrak{g}_{1}^{\sharp}+\mathfrak{g}_{1}^{\dagger}=\mathfrak{g}_{1}$ by part (a), we get $\mathcal{M}\left(\mathfrak{g}^{\sharp}\right)_{-2}=0$. Our final remark in part (b) then yields $\mathfrak{g}_{-2}=\widehat{\mathfrak{g}}_{-2}$. If $\widehat{\mathfrak{g}} \cong W(m ; \underline{1})$, this gives the result. If $\widehat{\mathfrak{g}} \cong K(2 m+1 ; \underline{1})^{(1)}$, then $\mathcal{M}\left(\mathfrak{g}^{\dagger}\right)_{-3}=\mathcal{M}\left(\mathfrak{g}^{\sharp}\right)_{-3}=\mathfrak{g}_{-3}$ by our remarks above.

(d) Suppose $\mathfrak{g}_{-3} \neq 0$. We have $\left[\mathfrak{g}_{-3}, \mathfrak{g}_{1}^{\sharp}\right]=0$ by part $(\mathrm{b})$, and $\left[\mathfrak{g}_{-3}, \mathfrak{g}_{1}\right]=$ $\left[\mathfrak{g}_{-3}, \mathfrak{g}_{1}^{\dagger}\right]=\widehat{\mathfrak{g}}_{-2}$ by part (c) and 1-transitivity. Since $\operatorname{dim} \widehat{\mathfrak{g}}_{-2}=1$ and $\mathfrak{g}$ is 1 -transitive, the $\mathfrak{g}_{0}^{(1)}$-module $\mathfrak{g}_{-3}$ embeds into $\operatorname{Hom}\left(\mathfrak{g}_{1}^{\dagger}, \mathfrak{g}_{-2}\right) \cong\left(\mathfrak{g}_{1}^{\dagger}\right)^{*}$. Moreover, because $\widehat{\mathfrak{g}} \cong K(2 m+1 ; \underline{1})^{(1)}$ in the present case, we have that $\mathfrak{g}_{0}^{(1)}=\mathfrak{s p}(V)$ and $\mathfrak{g}_{1}^{\dagger} \cong S^{3}(V)$ as $\mathfrak{g}_{0}^{(1)}$-modules; see part (a). Since $p>3$, Proposition 2.77 shows that $\mathfrak{g}_{1}^{\dagger}$ is an irreducible (and self-dual) $\mathfrak{g}_{0}^{(1)}$-module. It is generated by $D_{K}\left(x_{1}^{(3)}\right)$, a $\mathfrak{b}^{+}$-primitive vector of weight $3 \varpi_{1}$. Let $f^{3 \varpi_{1}}$ be a $\mathfrak{b}^{+}$-primitive vector of $\mathfrak{g}_{-3}$, and let $e^{-3 \varpi_{1}}$ and $e^{-\varpi_{1}}$ be $\mathfrak{b}^{-}$-primitive vectors of $\mathfrak{g}_{1}^{\dagger}$ and $\mathfrak{g}_{1}^{\sharp}$, respectively. Set $w:=\left[f^{3 \varpi_{1}}, e^{-3 \varpi_{1}}\right]$. By the above, both $\mathfrak{g}_{-3}$ and $\mathfrak{g}_{1}^{\sharp}$ are irreducible over $\mathfrak{g}_{0}^{(1)}$. Since $\left[\mathfrak{g}_{-3}, \mathfrak{g}_{1}^{\sharp}\right]=0$, we have $\left[f^{3 \varpi_{1}}, e^{-\varpi_{1}}\right]=0$, while the 1-transitivity of $\mathfrak{g}$ implies that $w$ spans $\mathfrak{g}_{-2}=$ $\widehat{\mathfrak{g}}_{-2}$. By our discussion in part (a), $\widehat{\mathfrak{g}}^{\sharp}$ is a Lie algebra of type $\mathrm{C}_{m+1}$. Because $\left[\left[e_{-2 \varepsilon_{1}}, e_{\varepsilon_{1}-\varepsilon_{2}}\right], e_{\varepsilon_{1}-\varepsilon_{2}}\right] \neq 0$ in any Lie algebra of type $\mathrm{C}_{m+1}$ over $\mathbb{F}$ in which $\widehat{\mathfrak{g}}_{-2}=\mathbb{F} e_{-2 \varepsilon_{1}}$, it must be that

$$
\left[f^{3 \varpi_{1}},\left[\left[e^{-3 \varpi_{1}}, e^{-\varpi_{1}}\right], e^{-\varpi_{1}}\right]\right]=\left[\left[w, e^{-\varpi_{1}}\right], e^{-\varpi_{1}}\right] \in \mathbb{F}^{\times} e_{-\alpha},
$$

where $\alpha$ is the highest root of $\Phi$. It is now immediate that the elements $f^{3 \varpi_{1}}$ and $\left[\left[e^{-3 \varpi_{1}}, e^{-\varpi_{1}}\right], e^{-\varpi_{1}}\right]$ satisfy all of the conditions of Theorem 4.4, contrary to the fact that $S^{3}(V) \neq V$ as $\mathfrak{s p}(V)$-modules. Therefore, $\mathfrak{g}_{-3}=0$.

(e) We turn now to the case in which $\widehat{\mathfrak{g}} \cong W(m ; \underline{1})$, where $m+1 \equiv 0$ mod $p$. It is much more complicated. As above, we let $\widehat{\mathfrak{g}}^{\dagger}$ denote the graded subalgebra of $\mathfrak{g}$ generated by $\mathfrak{g}_{-1} \oplus \mathfrak{g}_{0} \oplus \mathfrak{g}_{1}^{\dagger}$. Note that in the present case 
$m \geq 4$. Theorem 2.69 shows that the $\mathfrak{g}_{0}$-module $\mathfrak{g}_{1}$ has a unique composition series $\mathfrak{g}_{1} \supset \mathfrak{g}_{1}^{\dagger} \supset \mathfrak{g}_{1}^{\sharp} \supset 0$. Furthermore, $\mathfrak{g}_{1} / \mathfrak{g}_{1}^{\dagger} \cong \mathfrak{g}_{-1}^{*}$, and $\widehat{\mathfrak{g}}^{\dagger}$ is isomorphic to a graded Lie algebra $S(m ; \underline{1})^{(1)} \oplus \mathbb{F} \mathfrak{D}_{1}$; see Theorem 2.66. Setting $\mathfrak{g}^{\dagger}$ as above and using Lemma 4.30 , we see now that $\mathfrak{g}_{-2}=\mathcal{M}\left(\mathfrak{g}^{\dagger}\right)_{-2}$. In other words, $\left[\mathfrak{g}_{-2}, \mathfrak{g}_{1}^{\dagger}\right]=0$. By 1-transitivity, $\mathfrak{g}_{-2}$ is then isomorphic to a submodule of the $\mathfrak{g}_{0}$-module $\operatorname{Hom}\left(\mathfrak{g}_{1} / \mathfrak{g}_{1}^{\dagger}, \mathfrak{g}_{-1}\right) \cong \mathfrak{g}_{-1} \otimes \mathfrak{g}_{-1}$. Since $\mathfrak{g}_{0} \cong \mathfrak{g l}(V)$ and $\mathfrak{g}_{-1} \cong V^{*}$, it follows that $\mathfrak{g}_{-2}$ is isomorphic to a $\mathfrak{g l}(V)$-submodule of $V^{*} \otimes V^{*} \cong S^{2}\left(V^{*}\right) \oplus$ $\wedge^{2} V^{*}$. In particular, $\mathfrak{g}_{-2}$ is a completely reducible $\mathfrak{g}_{0}$-module.

(f) Suppose $\mathfrak{g}_{-2} \neq 0$ and let $\mathfrak{g}_{-2}^{\prime}$ be an irreducible $\mathfrak{g}_{0}$-submodule of $\mathfrak{g}_{-2}$. Clearly, either $\mathfrak{g}_{-2}^{\prime} \cong S^{2}\left(V^{*}\right)$ or $\mathfrak{g}_{-2}^{\prime} \cong \wedge^{2} V^{*}$. Due to the irreducibility and 1-transitivity of $\mathfrak{g}$, we have $\left[\mathfrak{g}_{-2}^{\prime}, \mathfrak{g}_{1}\right]=\mathfrak{g}_{-1}$. Therefore, $\left[\mathfrak{g}_{-2}^{\prime},\left[\mathfrak{g}_{1}, \mathfrak{g}_{1}^{\dagger}\right]\right]=$ $\left[\mathfrak{g}_{-1}, \mathfrak{g}_{1}^{\dagger}\right] \neq 0$ by transitivity. As a consequence, $\left[\mathfrak{g}_{-2}^{\prime}, \widehat{\mathfrak{g}}_{2}\right] \neq 0$.

Since $m+2 \not \equiv 0 \bmod p$, Theorem 2.69 shows that $\widehat{\mathfrak{g}}_{2}=\left(\widehat{\mathfrak{g}}_{2}\right)^{\sharp} \oplus\left(\widehat{\mathfrak{g}}_{2}\right)^{\dagger}$, where both $\left(\widehat{\mathfrak{g}}_{2}\right)^{\sharp}$ and $\left(\widehat{\mathfrak{g}}_{2}\right)^{\dagger}$ are irreducible $\mathfrak{g}_{0}^{(1)}$-modules. Moreover, $\left(\widehat{\mathfrak{g}}_{2}\right)^{\sharp}$ and $\left(\widehat{\mathfrak{g}}_{2}\right)^{\dagger}$ are generated by $\mathfrak{b}^{-}$-primitive vectors $e^{-2 \Lambda}$ and $e^{-\mu}$, respectively, where $\mu=2 \Lambda+\alpha, \Lambda=\varpi_{m-1}$, and $\alpha=\alpha_{1}+\cdots+\alpha_{m-1}=\varpi_{1}+\varpi_{m-1}$; see Theorem 2.69, Lemma 2.78, and [Bou1]. Let $f$ be a $\mathfrak{b}^{+}$-primitive vector of $\mathfrak{g}_{-2}^{\prime}$. Since $\mathfrak{g}_{-2}^{\prime}$ is isomorphic to a submodule of $S^{2}\left(V^{*}\right) \oplus \wedge^{2} V^{*}$, the weight of $f$ is equal to either $2 \Lambda$ or $\varpi_{m-2}$. Since $\left[\mathfrak{g}_{-2}^{\prime}, \widehat{\mathfrak{g}}_{2}\right] \neq 0$, it follows that either $\left[f, e^{-2 \Lambda}\right] \neq 0$ or else $\left[f, e^{-\mu}\right] \neq 0$. Applying Theorem 4.4 shows that the case $\left[f, e^{-\mu}\right] \neq 0$ is impossible. Thus $f$ has weight $2 \Lambda$, and $\left[f, e^{-\mu}\right]=0$. This, in turn, yields $\mathfrak{g}_{-2}=\mathfrak{g}_{-2}^{\prime} \cong S^{2}\left(V^{*}\right)$.

At this point, it will be convenient to identify $\widehat{\mathfrak{g}}$ with the graded Lie algebra $W(m ; \underline{1})$, adopt the notation of Section 2.13 , and choose $\mathfrak{t}$ to be the Cartan subalgebra $\mathfrak{h}$ from the proof of Theorem 2.69. The above shows that $\left[f, e^{-2 \Lambda}\right]$ is a nonzero element in $\mathfrak{h}$. Let $S^{\{2\}}$ denote the graded Lie subalgebra of $\mathfrak{g}$ generated by $\mathfrak{g}_{-2}, \mathfrak{g}_{0}$, and $\left(\widehat{\mathfrak{g}}_{2}\right)^{\sharp}$. Let $I$ be the maximal graded ideal of $S^{\{2\}}$ contained in the subspace $\bigoplus_{|i|>4} S_{i}^{\{2\}}$, and set $\bar{S}^{\{2\}}:=S^{\{2\}} / I$. Then $\bar{S}_{0}^{\{2\}} \cong \mathfrak{g}_{0}$ and both $\bar{S}_{-2}^{\{2\}}$ and $\bar{S}_{2}^{\{2\}}$ are irreducible $\bar{S}_{0}^{\{2\}}$-modules. Applying Theorem 3.20 we derive that $\bar{S}^{\{2\}}$ is isomorphic to a classical Lie algebra with a standard grading. Our earlier remarks show that the Dynkin diagram of $\bar{S}^{\{2\}}$ must have the following form:

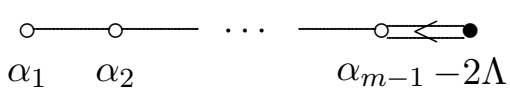

Therefore, it can be assumed, after rescaling $f$ if necessary, that $e^{-2 \Lambda}=$ $x_{m}^{(2)} \mathfrak{D}_{1}$ and $\left[f, e^{-2 \Lambda}\right]=h_{2 \Lambda}$, where $\Lambda\left(h_{2 \Lambda}\right)=1, \alpha_{m-1}\left(h_{2 \Lambda}\right)=-1$, and $\alpha_{i}\left(h_{2 \Lambda}\right)=0$ for $i \leq m-2$. 
(g) By Theorem 2.69 the $\mathfrak{g}_{0}^{(1)}$-module $\mathfrak{g}_{1}^{\sharp}$ is generated by a $\mathfrak{b}^{-}$-primitive vector $e^{-\Lambda}=x_{m}^{(1)} \mathfrak{D}_{1}$. Since $\mathfrak{g}_{1}^{\sharp} \subset \mathfrak{g}_{1}^{\dagger}$ and $\left[\mathfrak{g}_{-2}, \mathfrak{g}_{1}^{\dagger}\right]=0$ by part (e), we have

$$
\left[f, e^{-\Lambda}\right]=0 \text {. }
$$

Since $m+3 \not \equiv 0 \bmod p$, Theorem 2.69 shows that the $\mathfrak{g}_{0}^{(1)}$-module $\widehat{\mathfrak{g}}_{3}$ is a direct sum of its irreducible submodules $\left(\widehat{\mathfrak{g}}_{3}\right)^{\sharp}$ and $\left(\widehat{\mathfrak{g}}_{3}\right)^{\dagger}$. Moreover, the $\mathfrak{g}_{0}^{(1)}$-module $\left(\widehat{\mathfrak{g}}_{3}\right)^{\sharp}$ is generated by a $\mathfrak{b}^{-}$-primitive vector $e^{-3 \Lambda}:=x_{m}^{(3)} \mathfrak{D}_{1}$. In view of (2.68), we have

$$
\left[x_{m}^{(i)} \mathfrak{D}_{1}, x_{m}^{(j)} \mathfrak{D}_{1}\right]=(j-i)\left(\begin{array}{c}
i+j \\
i
\end{array}\right) x_{m}^{(i+j)} \mathfrak{D}_{1} \quad(\forall i, j \in \mathbb{N}) .
$$

Since $\operatorname{div}\left(x_{m}^{(3)} D_{m}-x_{m}^{(2)} \mathfrak{D}_{1}\right)=-(m+1) x_{m}^{(2)}=0$ and $\left(\widehat{\mathfrak{g}}_{2}\right)^{\dagger}$ consists of all divergence-free derivations in $\widehat{\mathfrak{g}}_{2}=W(m ; \underline{1})_{2}$, it must be that $-x_{m}^{(3)} D_{m}+$ $x_{m}^{(2)} \mathfrak{D}_{1} \in\left(\widehat{\mathfrak{g}}_{2}\right)^{\dagger}$. Since the irreducible $\mathfrak{g}_{0}^{(1)}$-module $\left(\widehat{\mathfrak{g}}_{3}\right)^{\dagger}$ contains $x_{m}^{(4)} D_{1}$ and

$$
\left[x_{m}^{(1)}\left(x_{1}^{(1)} D_{1}-x_{2}^{(1)} D_{2}\right),\left[x_{m}^{(1)}\left(x_{1}^{(1)} D_{1}-x_{2}^{(1)} D_{2}\right), x_{m}^{(2)} D_{1}\right]\right] \in \mathbb{F}^{\times} x_{m}^{(4)} D_{1},
$$

we have the inclusion $\left(\widehat{\mathfrak{g}}_{3}\right)^{\dagger} \subset \mathfrak{g}^{\dagger}$ which shows that

$$
\left[f, x_{m}^{(3)} D_{m}-x_{m}^{(2)} \mathfrak{D}_{1}\right]=0
$$

since, as we noted in part (e), $\left[\mathfrak{g}_{-2}, \mathfrak{g}_{1}^{\dagger}\right]=0$.

Set $f^{\Lambda}:=D_{m}$, a $\mathfrak{b}^{+}$-primitive vector of the $\mathfrak{g}_{0}^{(1)}$-module $\mathfrak{g}_{-1}$. Combining (4.35), (4.36), (4.37) with the equality $\Lambda\left(h_{2 \Lambda}\right)=1$ we now obtain

$$
\begin{aligned}
{\left[\left[f, f^{\Lambda}\right],\left[e^{-2 \Lambda}, e^{-\Lambda}\right]\right] } & =\left[\left[f,\left[e^{-2 \Lambda}, e^{-\Lambda}\right]\right], f^{\Lambda}\right]+\left[f,\left[f^{\Lambda},\left[e^{-2 \Lambda}, e^{-\Lambda}\right]\right]\right] \\
& =\left[\left[h_{2 \Lambda}, e^{-\Lambda}\right], f^{\Lambda}\right]+\left[f,\left[D_{m},\left[x_{m}^{(2)} \mathfrak{D}_{1}, x_{m}^{(1)} \mathfrak{D}_{1}\right]\right]\right] \\
& =-\Lambda\left(h_{2 \Lambda}\right)\left[e^{-\Lambda}, f^{\Lambda}\right]-3\left[f,\left[D_{m}, x_{m}^{(3)} \mathfrak{D}_{1}\right]\right] \\
& =\left[D_{m}, x_{m}^{(1)} \mathfrak{D}_{1}\right]-3\left[f, x_{m}^{(2)} \mathfrak{D}_{1}+x_{m}^{(3)} D_{m}\right] \\
& =\mathfrak{D}_{1}+x_{m}^{(1)} D_{m}-3\left[f, 2 x_{m}^{(2)} \mathfrak{D}_{1}+x_{m}^{(3)} D_{m}-x_{m}^{(2)} \mathfrak{D}_{1}\right] \\
& =\mathfrak{D}_{1}+x_{m}^{(1)} D_{m}-6 h_{2 \Lambda} .
\end{aligned}
$$

Since $\Lambda\left(h_{2 \Lambda}\right)=1$, we have $\left[\mathfrak{D}_{1}+x_{m}^{(1)} D_{m}-6 h_{2 \Lambda}, D_{m}\right]=-8 D_{m} \neq 0$. Consequently, $\left[f, f^{\Lambda}\right] \neq 0$ is a $\mathfrak{b}^{+}$-primitive vector of weight $3 \Lambda=3 \varpi_{m-1}$ in $\mathfrak{g}_{-3}$.

(h) Let $\mathfrak{g}_{-3}^{\prime}$ be the $\mathfrak{g}_{0}$-submodule of $\mathfrak{g}_{-3}$ generated by $\left[f, f^{\Lambda}\right]$, and denote by $S^{\{3\}}$ the Lie subalgebra of $\mathfrak{g}$ generated by $\mathfrak{g}_{-3}^{\prime}, \mathfrak{g}_{0}$, and $\left(\widehat{\mathfrak{g}}_{3}\right)^{\sharp}$. We give $S^{\{3\}}$ a $\mathbb{Z}$-grading by setting $S_{i}^{\{3\}}=\mathfrak{g}_{-3 i} \cap S^{\{3\}}$ for all $i \in \mathbb{Z}$. Let $J$ be the maximal graded ideal of $S^{\{3\}}$ contained in the subspace $\bigoplus_{|i| \geq 1} S_{i}^{\{3\}}$, and set $\bar{S}^{\{3\}}:=S^{\{3\}} / J$. Our computation in part (g) implies that $\left[f, f^{\lambda}\right] \notin J$. From this it is easy to deduce that the graded Lie algebra $\bar{S}^{\{3\}}$ is irreducible, transitive, and 1-transitive. Obviously, $\bar{S}_{0}^{\{3\}} \cong \mathfrak{g}_{0}$. 
Let $\Lambda^{\prime}=-w_{0}(\Lambda)=\varpi_{1}$ and extend $\Lambda^{\prime}$ to a linear function on $\mathfrak{h}$ by setting $\Lambda\left(\mathfrak{D}_{1}\right)=1$. Let $u^{3 \Lambda^{\prime}}$ denote a $\mathfrak{b}^{+}$-primitive vector of $\bar{S}_{-1}^{\{3\}} \cong S^{3}(V)$, and let $v^{\lambda}$ be any $\mathfrak{b}^{-}$-primitive vector of $\bar{S}_{1}^{\{3\}}$. Then $\left[u^{3 \Lambda^{\prime}}, v^{\lambda}\right] \neq 0$ by transitivity. If $\lambda \neq-3 \Lambda^{\prime}$, then Proposition 4.14 says that $\lambda$ does not vanish on $\mathfrak{h} \cap \mathfrak{g}_{0}^{(1)}$. Since $3 \varpi_{1} \notin\left\{\varpi_{1}, \varpi_{m-1}, 2 \varpi_{1}+\varpi_{m-1}, \varpi_{1}+2 \varpi_{m-1}\right\}$, this contradicts Theorem 4.4. Thus, the $\mathfrak{b}^{-}$-primitive vectors of $\bar{S}_{1}^{\{3\}}$ must have weight $-3 \Lambda^{\prime}$. Let $M$ be an irreducible $\mathfrak{g}_{0}$-submodule of $\bar{S}_{1}^{\{3\}}$. Since $\bar{S}_{-1}^{\{3\}}$ is a restricted $\mathfrak{g}_{0}^{(1)}$-module, so is the $\mathfrak{g}_{0}^{(1)}$-module $M \hookrightarrow \operatorname{Hom}\left(\bar{S}_{-1}^{\{3\}}, \mathfrak{g}_{0}\right)$. The preceding remark then implies that $M$ is generated by a $\mathfrak{b}^{-}$-primitive vector of weight $-3 \Lambda^{\prime}$. In this situation, Theorem 4.7(ii) shows that $\bar{S}_{1}^{\{3\}}$ is an irreducible $\mathfrak{g}_{0}^{(1)}$-module. But then $\bar{S}^{\{3\}}$ satisfies all of the conditions of Theorem 3.22. It follows that $\bar{S}^{\{3\}}$ is a classical graded Lie algebra whose null component is of type $\mathrm{A}_{m-1}$, $m \geq 4$. Yet $3 \varpi_{1}$ is not listed in (4.31), which exhibits all possible weights of $\mathfrak{b}^{+}$-primitive vectors in $\bar{S}_{-1}^{\{3\}}$ that can occur in this situation. This implies that in the present case $\mathfrak{g}_{-2}=0$, completing the proof.

\subsection{The case that $\mathfrak{g}_{0}$ is abelian}

Here we verify that $\mathfrak{g}_{0}$ can be abelian only in a Zassenhaus Lie algebra $W(1 ; \underline{n})$ or in $\mathfrak{s l}_{2}$. We use the fact that by transitivity, $\mathfrak{g}_{0}$ must be onedimensional, so that by irreducibility, $\mathfrak{g}_{-1}$ must be one-dimensional as well.

Lemma 4.38. Let $\mathfrak{g}=\bigoplus_{i=-q}^{r} \mathfrak{g}_{i}$ be a finite-dimensional graded Lie algebra which satisfies conditions (1)-(5), and suppose further that $\mathfrak{g}_{0}^{(1)}=0$. If $r>1$, then $q=1$, and $\mathfrak{g}$ is isomorphic to a graded Lie algebra $W(1 ; \underline{n})$ for some $n \geq 1$. If $q>1$, then $r=1$, and $\mathfrak{g}=\bigoplus_{i=-1}^{q} \mathfrak{g}_{-i}$ is isomorphic to a graded Lie algebra $W\left(1 ; \underline{n}^{\prime}\right)$ ) for some $n^{\prime} \geq 1$. If $r=q=1$, then $\mathfrak{g} \cong \mathfrak{s l}_{2}$.

Proof. Since $\mathfrak{g}_{0}$ is abelian, conditions (3) and (4) show that $\mathfrak{g}_{0}$ and $\mathfrak{g}_{-1}$ are one-dimensional, say $\mathfrak{g}_{0}=\mathbb{F} z_{0}$, and $\mathfrak{g}_{-1}=\mathbb{F} z_{-1}$, and $z_{0}$ acts as a nonzero scalar on $\mathfrak{g}_{-1}$. Since the pairing $\mathfrak{g}_{1} \times \mathfrak{g}_{-1} \rightarrow \mathfrak{g}_{0}$ given by the product is nondegenerate by transitivity, $\mathfrak{g}_{1} \cong \mathfrak{g}_{-1}^{*}$. Thus $\mathfrak{g}_{1}$ is one-dimensional also, say $\mathfrak{g}_{1}=\mathbb{F} z_{1}$.

Denote by $\mathfrak{g}^{\geq-1}$ and $\mathfrak{g}^{\leq 1}$ the graded subalgebras of $\mathfrak{g}$ generated by all $\mathfrak{g}_{i}$ with $i \geq-1$ and $i \leq 1$, respectively. Both $\mathfrak{g}^{\geq-1}$ and $\mathfrak{g}^{\leq 1}$ satisfy conditions (1)-(5). If $\mathfrak{g}=\mathfrak{g}^{\geq-1}$ or $\mathfrak{g}=\mathfrak{g}^{\leq 1}$, then our statement follows from Theorem 2.66. Thus, in what follows we will assume that $\min (r, q)>1$.

As $\left[\mathfrak{g}_{-1}, \mathfrak{g}_{-1}\right]=\left[\mathfrak{g}_{1}, \mathfrak{g}_{1}\right]=0$, Theorem 2.66 shows that $\mathfrak{g}^{\geq-1} \cong W(1 ; \underline{n})$ and $\mathfrak{g}^{\leq 1} \cong W\left(1 ; \underline{n}^{\prime}\right)$ for some positive integers $n$ and $n^{\prime}$. In particular, 
$\operatorname{dim} \mathfrak{g}_{i}=1$ for $-q \leq i \leq r$, and $\left[\mathfrak{g}_{1}, \mathfrak{g}_{p-2}\right]=0=\left[\mathfrak{g}_{-1}, \mathfrak{g}_{-(p-2)}\right]$. For $-p+2 \leq$ $i \leq p-2$, choose $z_{i} \in \mathfrak{g}_{i} \backslash\{0\}$. By transitivity, $\left(\operatorname{ad} z_{-1}\right)^{p-3}\left(z_{p-2}\right) \neq 0$, while

$$
\left(\operatorname{ad} z_{-1}\right)^{p-3}\left(\left[\mathfrak{g}_{-(p-2)}, \mathfrak{g}_{p-2}\right]\right) \subseteq \mathbb{F}\left(\operatorname{ad} z_{-1}\right)^{p-3}\left(z_{0}\right)=0,
$$

since $p \geq 5$. But then

$$
\begin{aligned}
0 & =\left(\operatorname{ad} z_{-1}\right)^{p-3}\left(\left[\mathfrak{g}_{-(p-2)}, z_{p-2}\right]\right) \\
& =\left[\mathfrak{g}_{-(p-2)},\left(\operatorname{ad} z_{-1}\right)^{p-3}\left(z_{p-2}\right)\right] \\
& =\left[\mathfrak{g}_{-(p-2)}, \mathfrak{g}_{1}\right],
\end{aligned}
$$

violating condition (5). Therefore, $\min (r, q)=1$, proving the lemma.

\subsection{Completion of the proof of the Main Theorem}

Suppose $\mathfrak{g}=\bigoplus_{j=-q}^{r} \mathfrak{g}_{j}$ is a graded Lie algebra satisfying the hypotheses of the Main Theorem, and let $\widehat{\mathfrak{g}}$ denote the Lie subalgebra of $\mathfrak{g}$ generated by $\mathfrak{g}_{-1} \oplus \mathfrak{g}_{0} \oplus \mathfrak{g}_{1}$. Note that $\widehat{\mathfrak{g}}$ satisfies the hypotheses of the Main Theorem. If $\mathfrak{g}_{0}^{(1)}=0$, then the Main Theorem follows from Lemma 4.38. So assume from now that $\mathfrak{g}_{0}^{(1)} \neq 0$. Since $\left[\left[\mathfrak{g}_{-1}, \mathfrak{g}_{1}\right], \mathfrak{g}_{1}\right] \neq 0$ by 1 -transitivity, Theorem 1.63 shows $\mathfrak{g}_{-1}$ is a restricted $\mathfrak{g}_{0}^{(1)}$-module. Since $\mathfrak{g}_{1}$ embeds into $\operatorname{Hom}\left(\mathfrak{g}_{-1}, \mathfrak{g}_{0}\right)$ by transitivity, it is a restricted $\mathfrak{g}_{0}^{(1)}$-module too.

Let $f^{\Lambda}$ be a $\mathfrak{b}^{+}$-primitive vector of weight $\Lambda$ in the $\mathfrak{g}_{0}$-module $\mathfrak{g}_{-1}$, and let $e^{\Gamma}$ be a $\mathfrak{b}^{-}$-primitive vector of weight $\Gamma$ in the $\mathfrak{g}_{0}$-module $\mathfrak{g}_{1}$. Then $\left[f^{\Lambda}, e^{\Gamma}\right] \neq 0$ by transitivity. If $\left[f^{\Lambda}, e^{\Gamma}\right] \notin \mathfrak{t}$, then it can be assumed (after rescaling if necessary) that $\left[f^{\Lambda}, e^{\Gamma}\right]=e_{-\alpha}$ for some $\alpha \in \Phi$. If $\alpha$ is a positive root, then Theorem 4.18 shows that $\widehat{\mathfrak{g}}$ is a restricted Lie algebra of Cartan type with its natural grading. More precisely, $\widehat{\mathfrak{g}}$ is either $W(m ; \underline{1}), m \geq 2$, or $S(m ; \underline{1})^{(1)}, m \geq 3$, or $S(m ; \underline{1})^{(1)} \oplus \mathbb{F} \mathfrak{D}_{1}, m \geq 3$, or $H(2 m ; \underline{1})^{(2)}, m \geq 1$, or $H(2 m ; \underline{1})^{(2)} \oplus \mathbb{F} \mathfrak{D}_{1}, m \geq 1$, or $K(2 m+1 ; \underline{1})^{(1)}, m \geq 1$. In this situation, Lemmas 4.30 and 4.33 yield that $\mathfrak{g}$ is isomorphic to a Lie algebra of Cartan type with its natural grading.

If $\alpha$ is a negative root, then Lemma 4.28 says that $\mathfrak{g}_{1}$ is an irreducible $\mathfrak{g}_{0}$-module. In this case, we can regard $\mathfrak{g}$ with its reverse grading without violating the hypotheses of the Main Theorem. Interchanging the roles of positive and negative roots and arguing as above, we are now able to conclude that $\mathfrak{g}$ itself is isomorphic to a Lie algebra of Cartan type with the reverse grading.

Thus, from now on we can assume that $\left[f^{\Lambda}, e^{\Gamma}\right] \in \mathfrak{t}$ for any $\mathfrak{b}^{-}$-primitive vector $e^{\Gamma} \in \mathfrak{g}_{1}$. Clearly, this implies that any irreducible $\mathfrak{g}_{0}$-submodule of $\mathfrak{g}_{1}$ is generated by a $\mathfrak{b}^{-}$-primitive vector of weight $-\Lambda$. In this situation Theorem 4.7(ii) applies showing that $\mathfrak{g}_{1}$ is an irreducible $\mathfrak{g}_{0}$-module. But then Theorem 3.22 says that either $\mathfrak{g}$ is a classical Lie algebra with one of its standard gradings or else $p=5$ and $\mathfrak{g}$ is a Melikyan algebra with its natural grading or the reverse of its natural grading. 


\section{Bibliography}

[B] G.M. Benkart, The classification of the simple modular Lie algebras. VI. Solving the final case by Helmut Strade. Mathematical Reviews Featured Review, 98j: 17020 .

[BG] G.M. Benkart and T.B. Gregory, Graded Lie algebras with classical reductive null component, Math. Ann. 285 (1989), 85-98.

[BGK] G. Benkart, T. Gregory, and M.I. Kuznetsov, On graded Lie algebras of characteristic three with classical reductive null component, The Monster and Lie Algebras, ed. by J. Ferrar and K. Harada, de Gruyter Berlin, New York (1998), 149-164.

[BKK] G.M. Benkart, A.I. Kostrikin, and M.I. Kuznetsov, The simple graded Lie algebras of characteristic three with classical reductive component $L_{0}$, Comm. Algebra 24 (1996), 223-234.

[BKM] G.M. Benkart, S.-J. Kang, and K.C. Misra, Graded Lie algebras of Kac-Moody type, Adv. Math. 97 (1993), 154-190.

[BO] G.M. Benkart and J.M. Osborn, Representations of rank one Lie algebras of characteristic p, Lie Algebras and Related Topics (Proceedings, New Brunswick, N.J., 1981), Lecture Notes in Math. 993, Springer-Verlag, 1982, 1-37.

[BW] R.E. Block and R.L. Wilson, Classification of the restricted simple Lie algebras, J. Algebra 14 (1988), 115-259.

[Bo] A. Borel, Linear Algebraic Groups, W.A. Benjamin, New York, 1969. Second ed.. Graduate Texts in Math. 126 Springer-Verlag, New York, 1991.

[Bou1] N. Bourbaki, Groupes et Algèbres de Lie, Chapitres IV-VI, Hermann, Paris, 1968. English transl., Lie groups and Lie algebras, Chapters 4-6. Elements of Mathematics, Springer-Verlag, Berlin, 2002.

[Bou2] N. Bourbaki, Groupes et Algèbres de Lie, Chapitres VII-IX, H 3 ermann, Paris, 1975. English transl., Lie groups and Lie algebras, Chapters 7-9, Elements of Mathematics, Springer-Verlag, Berlin, 2005.

[C] C.W. Curtis, Representations of Lie algebras of classical type with application to linear groups, J. Math. Mech. 9 (1960), 307-326.

[G1] T.B. Gregory, Simple Lie Algebras with Classical Simple Null Component, Yale University Ph.D. Dissertation, 1977.

[G2] T.B. Gregory, Simple Lie algebras with classical reductive null component, J. Algebra 63 (1980), 484-493.

[GK] T.B. Gregory and M.I. Kuznetsov, On graded Lie algebras of characteristic three with classical reductive null component, preprint.

[H1] J.E. Humphreys, Algebraic Groups and Modular Lie Algebras, Mem. Amer. Math. Soc. 71, Providence, RI, 1967.

[H2] J.E. Humphreys, Linear Algebraic Groups, Graduate Texts in Math. 21, SpringerVerlag, New York-Heidelberg, 1975; 5th printing 1998.

[H3] J.E. Humphreys, Introduction to Lie Algebras and Representation Theory, Graduate Texts in Math. 9 Springer-Verlag, New York-Berlin 1972; 7th printing 1997.

[J] N. Jacobson, Lie Algebras, Wiley Interscience, New York 1962. Reprinted, Dover Publ. Inc., New York, 1979. 
[Ja] J.C. Jantzen, Representations of Algebraic Groups, Pure and Applied Mathematics 131, Academic Press, New York, 1987. Second ed., Mathematical Surveys and Monographs 107 Amer. Math. Soc., Providence, RI, 2003.

[K1] V.G. Kac, Simple irreducible Lie algebras of finite growth, Izv. Akad. Nauk SSSR, Ser. Mat. 32 (1968), 1323-1367 (Russian), English transl., Math. USSR-Izv. 2 (1968), 1271-1331.

[K2] V.G. Kac, The classification of simple Lie algebras over a field of nonzero characteristic, Izv. Akad. Nauk SSSR, Ser. Mat. 34 (1970), 385-408, (Russian), English transl., Math. USSR-Izv., 4 (1970), 391-413.

[K3] V.G. Kac, A description of the filtered Lie algebras with which graded Lie algebras of Cartan type are associated. (Russian) Izv. Akad. Nauk SSSR Ser. Mat. 38 (1974), 800-834. Corrections: Izv. Akad. Nauk SSSR Ser. Mat. 40 (1976), no. 6, 1415.

[KO] A.I. Kostrikin and V.V. Ostrik, On a recognition theorem for Lie algebras of characteristic 3. (Russian) Mat. Sb. 186 (1995), no. 10, 73-88; translation in Sb. Math. 186 (1995), no. 10, 1461-1475.

[KS] A.I. Kostrikin and I.R. Shafarevich, Graded Lie algebras of finite characteristic, Izv. Akad. Nauk SSSR, Ser. Mat. 33 (1969), 251-322 (Russian), English transl., Math. USSR-Izv. 3 (1969), 237-304.

[Ku1] M.I. Kuznetsov, Modular filtered Lie algebras with unipotent derivation (Russian), III All-Union Symp. on Theory of Rings, Algebras, and Modules, Tartu (1976), thes. report, 61.

[Ku2] M.I. Kuznetsov, The Melikyan algebras as Lie algebras of the type $\mathrm{G}_{2}$, Comm. Algebra 19 (1991), 1281-1312.

[M] O. Mathieu, Classification des algèbres de Lie simples, Astérisque 266 (2000), 245-286.

[MS] W.H. Mills and G.B Seligman, Lie algebras of classical type, J. Math. Mech. 6 (1957), 519-548.

[P1] A.A. Premet, Lie algebras without strong degeneration, Mat. Sbornik 129(171) (1986), no. 1; Eng. transl., Math. USSR Sbornik 57(1987), no. 1, 151-164.

[P2] A.A. Premet, Current state of the classification problem for simple Lie algebras of characteristic $p>3$, a lecture at the conference "Groups and Representations" dedicated to the 60th birthday of Gary Seitz, March 24-27, 2004, University of Oregon, Eugene, Oregon.

[PS1] A.A. Premet and H. Strade, Simple Lie algebras of small characteristic I. Sandwich elements, J. Algebra 189 (1997), 419-480.

[PS2] A.A. Premet and H. Strade, Simple Lie algebras of small characteristic II. Exceptional roots, J. Algebra 216 (1999), 190-301.

[PS3] A.A. Premet and H. Strade, Simple Lie algebras of small characteristic III. The toral rank two case, J. Algebra 242 (2001), 236-337.

[PS4] A.A. Premet and H. Strade, Simple Lie algebras of small characteristic IV. Solvable and classical roots, J. Algebra 278 (2004), 766-833.

[PS5] A.A. Premet and H. Strade, Simple Lie algebras of small characteristic V. The non-Melikian case, in preparation.

[S] G.B. Seligman, Modular Lie Algebras, Ergebnisse der Mathematik und ihrer Grenzgebiete 40, Springer, New York, 1967.

[Sk1] S. Skryabin, Modular Lie algebras of Cartan type over algebraically non-closed fields, 19 (1991), 1629-1741.

[Sk2] S. Skryabin, On the structure of the graded Lie algebra associated with a noncontractible filtration, J. Algebra 197 (1997), no. 1, 178-230.

[Sp] T.A. Springer, Linear Algebraic Groups, 2nd ed., Progress in Mathematics, 9, Birkhäuser, Boston, 1998. 
[SF] H. Strade and R. Farnsteiner, Modular Lie Algebras and their Representations, Monographs and Textbooks in Pure and Applied Mathematics, 116 Marcel Dekker, Inc., New York, 1988.

[St] H. Strade, Simple Lie Algebras over Fields of Positive Characteristic, Volume 1: Structure Theory, DeGruyter Expositions in Math. 38, Berlin 2004.

[SW] H. Strade and R. L. Wilson, Classification of the simple Lie algebras over algebraically closed fields of prime characteristic, Bull. Amer. Math. Soc. 24 (1991), 357-362.

[W] B.J. Weisfeiler, On the structure of the minimal ideal of some graded Lie algebras in characteristic $p>0$, J. Algebra 53 (1978), 344-361.

[WK] B.J. Weisfeiler and V.G. Kac, The irreducible representations of Lie $p$-algebras, Funct. Anal. Appl. 5 (1971), 111-117.

[Wi1] R.L. Wilson, Automorphisms of graded Lie algebras of Cartan type Comm. Algebra 3(7) (1975), 591-613.

[Wi2] R.L. Wilson, A structural characterization of the simple Lie algebras of generalized Cartan type over fields of prime characteristic, J. Algebra 40 (1976), 418-465.

[Wi3] R.L. Wilson, The classification problem for simple Lie algebras of characteristic $p$. Algebra, Carbondale 1980 (Proc. Conf., Southern Illinois Univ., Carbondale, Ill., 1980), pp. 13-32, Lecture Notes in Math. 848, Springer, Berlin, 1981. 